\title{
Berechnung starr-plastischer Platten mittels finiter Elemente
}

\section{Working Paper}

\section{Author(s):}

Knöpfel, Hans

Publication date:

1973

Permanent link:

https://doi.org/10.3929/ethz-a-005756938

Rights / license:

In Copyright - Non-Commercial Use Permitted

Originally published in:

Bericht / Institut für Baustatik ETH Zürich 47 
(C) Birkhäuser Verlag Basel und Stuttgart, 1973 ISBN 3-7643-0689-0 


\title{
Berechnung starr-plastischer Platten mittels finiter Elemente
}

\author{
von \\ Dr. sc. techn. Hans Knöpfel \\ Institut für Baustatik \\ Eidgenössische Technische Hochschule Zürich
}


Leere SeitelnBlank 
Die vorliegende Arbeit wurde von Herrn H. Knöpfel als Dissertation (Referent Prof. Dr. B. Thürlimann, Korreferent PD Dr. E. Anderheggen) ausgearbeitet. Sie knüpft an frühere Arbeiten von R. Wolfensberger und E. Anderheggen an, welche ebenfalls an unserem Institut entstanden sind.

Damit hat eine systematische Entwicklungsarbeit unseres Institutes ihren Abschluss gefunden. Als ein Resultat dieser Arbeit liegt nun ein ComputerProgramm zur Berechnung der Traglast von Stahlbetonplatten vor, welches sich dank der einfachen Ein- und Ausgabe für die praktische Anwendung eignet. Wir erwarten, dass es in vielen Fällen Verwendung finden wird. 


\section{Leere SeitelnBlank}


2. Plastizitätsbedingungen 9

2.1 Linearisierte Fliessbedingung für Stahlbetonplatten 9

2.2 Besonderheiten 15

3. Ansätze für Momente und Verschiebungen 21

3.1 Einführung der Methode der finiten Elemente 21

3.2 Kriterien für die Approximationsfunktionen 28

3.3 Gewählte Ansätze 29

4. Lineare Momente und Verschiebungen 31

4.1 Ansätze und Bezeichnungen 31

4.2 Gleichgewichtsbedingungen 32

4.3 Mathematische Formulierung des Gleichgewichtes 41

4.4 Randbedingungen 43

4.5 Plastizitätsbedingungen 48

5. Konstante Momente und lineare Verschiebungen 50

5.1 Ansatz für die Momente 50

5.2 Gleichgewichtsbedingungen 52

5.3 Mathematische Formulierung des Gleichgewichtes 55

5.4 Plastizitätsbedingungen 55

6. Primales und duales Problem 59

6.1 Problemstellung 59

6.2 Kinematischer Aspekt der Fliessbedingung 62

6.3 Kontrolle des Mechanismus 65

6.4 Berechnung des Traglastfaktors 76

$\begin{array}{ll}\text { 7. Optimierungsverfahren } & 80\end{array}$

7.1 Aufgabe und Lösungsablauf 80

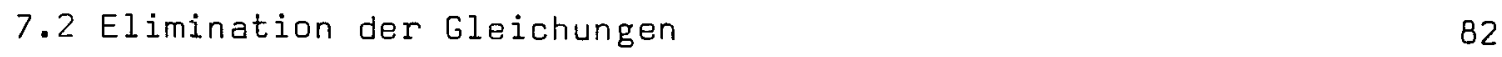

7.3 Elimination der übrigen freien Variablen 84

7.4 Optimierung 86

7.5 Duales Problem 90

7.6 Revidiertes Simplexverfahren 95 
$\begin{array}{ll}\text { 8. Anwendung auf praktische Beispiele } & 101\end{array}$

8.1 Computerprogramme 101

8.2 Eingespannte Quadratplatte 103

8.3 Kreisplatte 106

8.4 Unendlich ausgedehnte Flachdecke 107

8.5 Orthotrope, schiefe Armierung 110

Anhang A Tatsächliches Verhalten von Stahlbetonplatten 113

Anhang B Mathematische Formulierung des Gleichgewichtes 115

Anhang C Mechanismusmethode mit finiten Elementen 134

Anhang $D$ Hinweis auf die optimale Bemessung starr-plastischer Platten 140

$\begin{array}{ll}\text { Zusammenfassung } & 145\end{array}$

Resumé 146

$\begin{array}{ll}\text { Summary } & 147\end{array}$

$\begin{array}{ll}\text { Bezeichnungen } & 148\end{array}$

Literaturverzeichnis $\quad 150$ 
Das elastische Verhalten von Platten kann heute dank numerischer Verfahren (z.B. der Methode der finiten Elemente) weitgehend berechnet werden. Bei der Methode der finiten Elemente werden anstelle der exakten Funktionen für Schnittkräfte und Verschiebungen Näherungsfunktionen verwendet, welche in einem Teilbereich der Platte (Element) einen einfachen Verlauf haben. Der Verlauf kann durch eine endliche Anzahl von Parametern spezifiziert werden. Durch diese Diskretisation wird aus den Differentialgleichungen für Gleichgewicht, Verträglichkeit und Randbedingungen ein Gleichungssystem mit einer endlichen Anzahl von Unbekannten. Für praktische Aufgaben genügend genaue Resultate können mit einem befriedigenden Aufwand ermittelt werden.

Die Berechnung des elastischen Verhaltens befriedigt jedoch nicht, wenn die Sicherheit auf die Traglast bezogen werden soll. Es ist oft nicht wirtschaftlich oder nicht möglich, die Armierung genau den Schnittkräften bei elastischem Verhalten anzupassen. Weiter entstehen durch die Annahme, dass sich die Platten elastisch verhalten, Spannungsspitzen, die in Wirklichkeit durch plastisches Fliessen abgebaut werden können, ohne dass die Brauchbarkeit beeinträchtigt wird. Die Berücksichtigung des nichtelastischen Verhaltens ermöglicht also, einfachere Plattenkonstruktionen im Hoch-, Brücken- und Schutzraumbau zu verwenden.

Das unelastische Verhalten kann mittels der Methode der finiten Elemente berechnet werden, indem die Lasten schrittweise gesteigert und die Verschiebungen und Spannungen für jeden Schritt mit den zugehörigen Steifigkeitseigenschaften ermittelt werden. Damit kann der Zustand des Tragwerkes bei jeder Laststufe berechnet und beurteilt werden (z.B. Entwicklung des Rissbildes [20]). Diese Methode ist jedoch sehr aufwendig. Es wurden deshalb Näherungsverfahren entwickelt (z.B. das Verfahren der "initialen Spannungen" mit konstanter Steifigkeitsmatrix [19]).

Für die Berechnung des starr-plastischen Verhaltens von Stahlbetonplatten wird oft die Bruchlinien-Methode [9], [2], [21] verwendet. Diese Methode basiert auf dem Satz über den obern Grenzwert (jede Belastung, zu der sich ein instabiler kinematisch zulässiger Bewegungszustand angeben lässt, liegt nicht tiefer als die Traglast [4]). Es müssen also verschiedene Mechanismen (kinematisch zulässige Geschwindigkeitsfelder) untersucht werden mit dem Ziel, eine möglichst tiefe Last zu finden. Minimalbedingungen für einzelne geometrische Parameter können formuliert werden, aber die Methode ist nur für diejenigen Fälle gegen grobe Fehler gesichert, bei denen die Bruchlinienkonfiguration bereits bekannt ist (Versuche, Erfahrung). Der Wunsch nach 
unteren Grenzwerten der Traglast führte zu einem vereinfachten statisch zulässigen Gleichgewichtsmodell [1], für das mittels Optimierung ein unterer Grenzwert der Traglast bestimmt werden konnte. Es wurde dabei eine linearisierte Fliessfigur als Plastizitätsbedingung verwendet, damit die lineare Programmierung verwendet werden konnte.

Das Ziel dieser Arbeit ist, Berechnungsverfahren zu finden, mit denen die Traglast beliebiger starr-plastischer Platten zuverlässig und wirtschaftIich berechnet werden kann. Dazu wird die Methode der finiten Elemente benützt. Eine allgemein gültige, übersichtliche Darstellung des Problems und ein zweckmässiges Lösungsverfahren sollen gefunden werden. Einen Ueberblick über die theoretischen Grundlagen findet man in [3].

In dieser Arbeit wird hauptsächlich die Analyse der starr-plastischen Platten, d.h. die Bestimmung der Traglast bei bekannter Armierung, behandelt. Auf ein Verfahren für die optimale Bemessung wird im Anhang $D$ hingewiesen.

Für die grundlegenden Kenntnisse in der Berechnung des starr-plastischen Verhaltens von Platten [1], [4] sowie in linearer Programmierung [5], sei auf die Literatur verwiesen. Die hier verwendeten Bezeichnungen sind am Schluss zusammengestellt sowie bei ihrer Einführung definiert. Neben der üblichen Matrixschreibweise wird auch - wenn eine einfache Darstellung resultiert die Indexschreibweise benutzt, wobei partielle Ableitungen als untere Indices mit vorangehendem Komma $(\partial w / \partial n=w, n)$ geschrieben werden. Bei der Indexschreibweise wird die Summenkonvention für untere Indices benutzt $\left(\{a\}^{\top}\{b\}=a_{i} b_{i}\right)$.

Die folgende Zusammenfassung diene als Leberblick über die Arbeit. Am Anfang wird die Fliessbedingung für starr-plastisches Materialverhalten diskutiert (Kapitel 2). Dann wird das Problem nach dem statischen Grenzwertsatz berechnet. Voraus werden einige kennzeichnende Eigenschaften der Approximation durch finite Elemente zusammengefasst und das lineare Programm allgemein hergeleitet (Kapitel 3). Nachher werden die Gleichgewichts- und Plastizitätsbedingungen für zwei Modelle formuliert (Kapitel 4 und 5). Anschliessend wird gezeigt, dass das duale Programm die Berechnung nach dem kinematischen Grenzwertsatz enthält (Kapitel 6). Die Lösung der linearen Programme wird mittels eines speziellen Optimierungsverfahrens berechnet (Kapitel 7). Schliesslich werden die Computerprogramme und die Resultate der Berechnung praktischer Beispiele beschrieben (Kapitel 8 ). 


\subsection{Linearisierte Fliessbedingung für Stahlbetonplatten}

Als Grundlage wird die bekannte Fliessbedingung von Johansen [9] verwendet. Sie bezieht sich auf eine orthogonale Armierung und die zugehörigen plastischen Momente in einem bestimmten Bereich der Stahlbetonplatte.
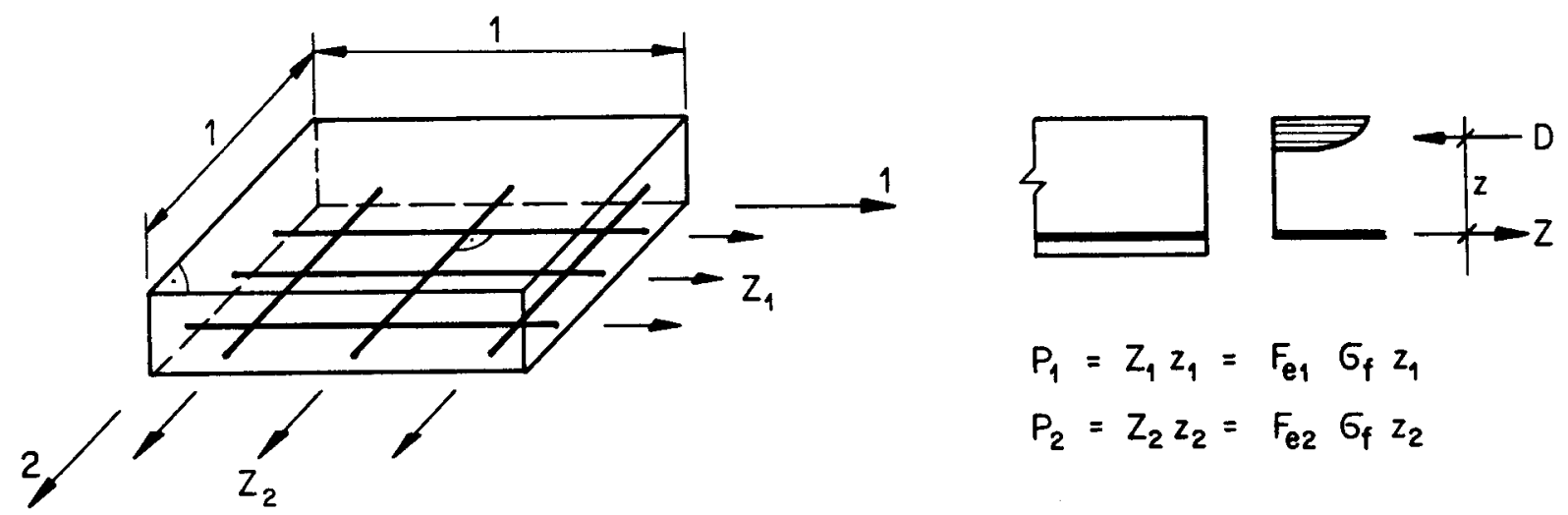

$$
\begin{aligned}
& P_{1}=Z_{1} z_{1}=F_{e 1} G_{f} z_{1} \\
& P_{2}=Z_{2} z_{2}=F_{e 2} \sigma_{f} z_{2}
\end{aligned}
$$

Bild 2.1: Positive plostische Hauptmomente

Im Bild 2.1 sind $F_{e 1}$ und $F_{e 2}$ die Querschnittsflächen der Armierungsstähle pro Einheitslänge, $\sigma_{f}$ ist die Fliessspannung der Armierungsstähle und $\mathbf{z}_{1}$ ist der Hebelarm für das positive plastische Hauptmoment $P_{1}$. Die negativen plastischen Hauptmomente $N_{1}$ und $N_{2}$ werden analog definiert. Es wird angenommen, dass keine Drillungsmomente in den Richtungen 1 und 2 existieren, weil die Zugkräfte keine Komponente parallel zur Schnittfläche haben.

Die Fliessbedingung in Funktion der Momente in Richtung der Armierungen hat eine rechteckige Form, d.h. die plastischen Momente der Platte in den beiden Hauptrichtungen hängen nicht voneinander ab.

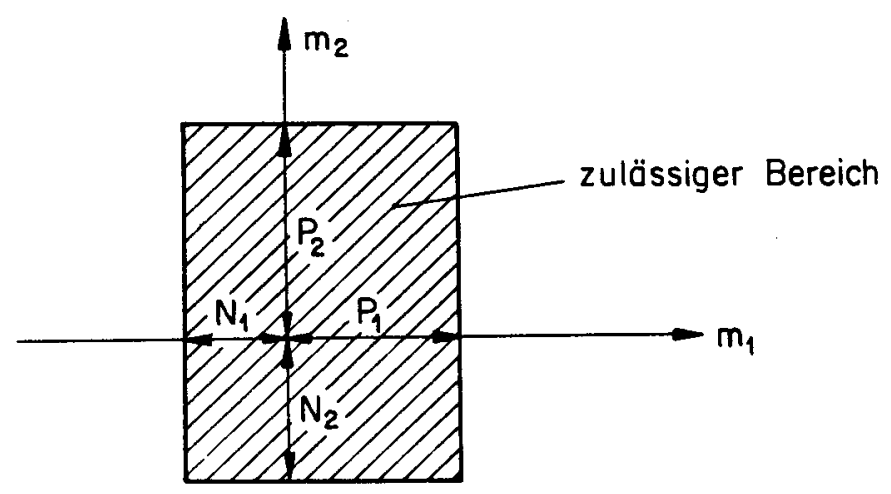

Bild 2.2: Johansen'sche Fliessbedingung 
Gesucht sei nun die Formulierung der Fliessbedingung für orthogonale Armierung im Koordinatensystem $(x-y)$. Die plastischen Widerstände lassen sich mittels Gleichgewichtsbedingungen an einem infinitesimalen Dreieckelement berechnen.
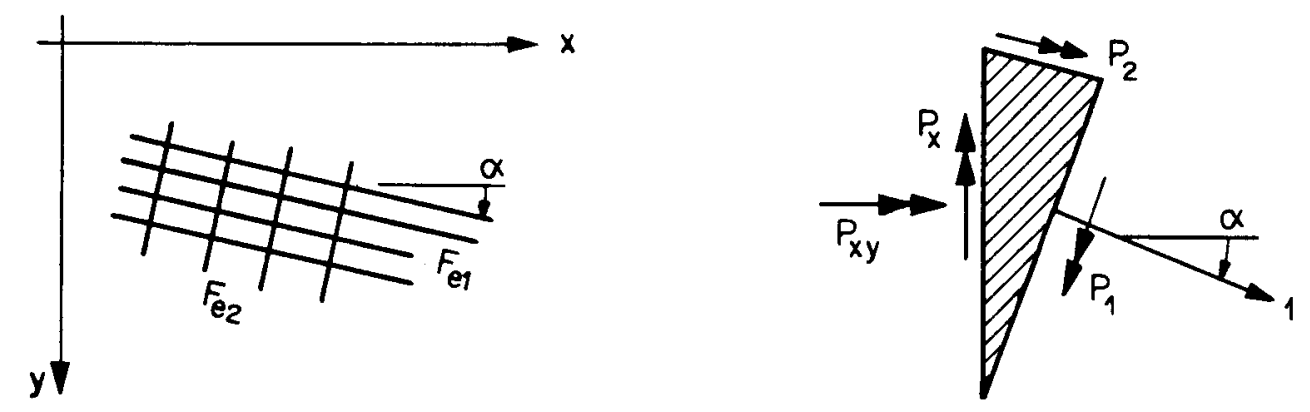

\section{Bild 2.3: Positive plostische Momente}

Aus Gleichgewichtsgründen ist

$$
\begin{aligned}
& P_{x}=P_{1} \cos ^{2} \alpha+P_{2} \sin ^{2} \alpha \\
& P_{y}=P_{1} \sin ^{2} \alpha+P_{2} \cos ^{2} \alpha \\
& P_{x y}=\frac{1}{2}\left(P_{1}-P_{2}\right) \sin 2 \alpha
\end{aligned}
$$

Die Widerstände $P_{x}$ und $P_{y}$ sind die Biegemomente, die im entsprechenden Schnitt aufgenommen werden können; $P_{x y}$ ist das Drillungsmoment, das zusätzlich dazu aufgenommen werden kann. Bei der Transformation setzt man starrplastisches Verhalten voraus. Die Fliessspannung ist also in allen Armierungsstäben erreicht, die einen plastifizierten Schnitt kreuzen. Eine Folge dieser Voraussetzung ist, dass z.B. nicht nur $m_{x}=P_{x}$, sondern auch $m_{x y}=P_{x y}$ ist, wenn der Schnitt senkrecht zur $x$-Axe plastifiziert ist. Diese Annahmen haben sich bei Versuchen als befriedigende Näherung für das wirkliche Verhalten erwiesen (siehe Anhang $A$ ).

Bis jetzt wurde vorausgesetzt, dass die Armierung orthogonal sei. Wenn die Armierung nicht orthogonal ist, können zunächst die Zugkräfte der Armierungsstäbe im Koordinatensystem $(x-y)$ berechnet werden. 


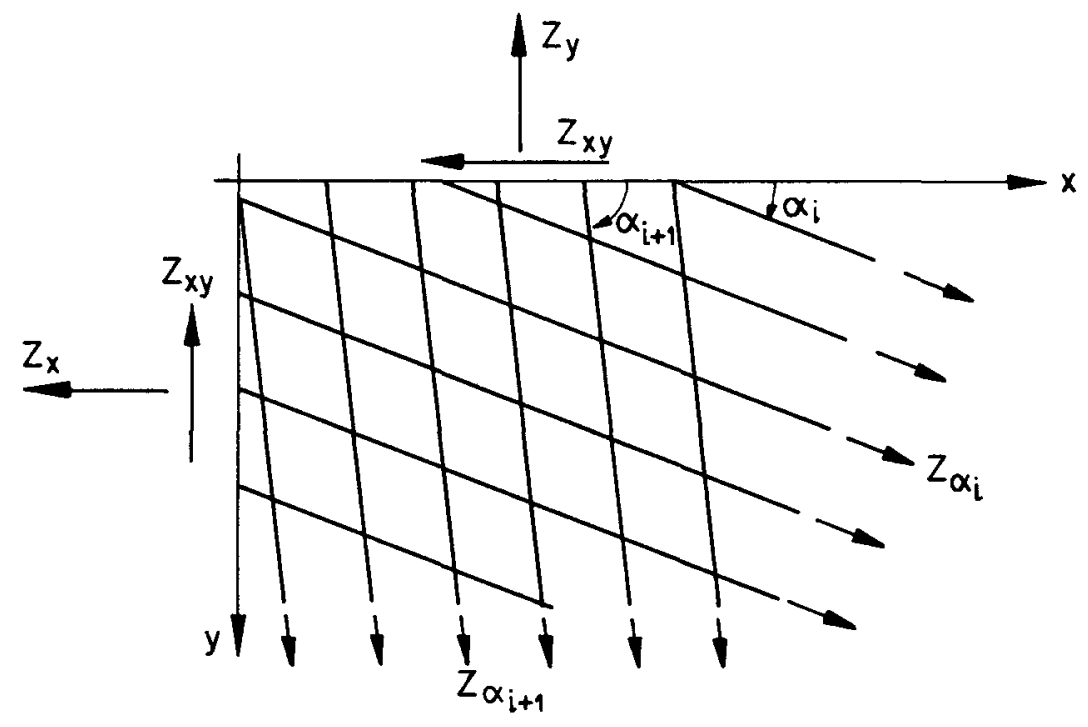

Bild 2.4: Armierung in beliebiger Richtung

Aus dem Bild 2.4 geht hervor, dass für $n$ Scharen von Armierungsstäben

$$
\begin{aligned}
& Z_{x}=\sum_{i=1}^{n} Z_{\alpha_{i}} \cos ^{2} \alpha_{i} \\
& z_{y}=\sum_{i=1}^{n} Z_{\alpha_{i}} \sin ^{2} \alpha_{i} \\
& z_{x y}=\sum_{i=1}^{n} z_{\alpha_{i}} \sin \alpha_{i} \cos \alpha_{i}
\end{aligned}
$$

ist. Alle Zugkräfte haben die Dimension $t / m$. Die Hauptrichtungen für den Zug- und Druckbereich der Platte sind bestimmt durch die Gleichung

$$
\tan 2 \varphi_{1,2}=\frac{2 Z_{x y}}{Z_{x}-Z_{y}}
$$

Aus den zugehörigen Hauptzugkräften $z_{1}$ und $z_{2}$ erhält man die plastischen Hauptmomente $P_{1}$ und $P_{2}$, womit die Armierung in beliebiger Richtung auf eine äquivalente orthogonale Armierung zurückgeführt ist.

Die Fliessbedingung für positive Momente ist erfültt, wenn für jede beliebige Richtung mit der Normalen $n$

$$
m_{n} \leqq P_{n}
$$

ist. Die Bezeichnung der Momente und die Richtung der Normalen sind im Bild 2.5 festgehalten. Die Vorzeichen der Momente wurden nach der folgenden Regel bestimmt: Momente sind positiv, wenn sie in der untersten Faser der 
Platte Spannungen erzeugen, die in die Axenrichtungen des mitlaufenden Koordinatensystems zeigen.

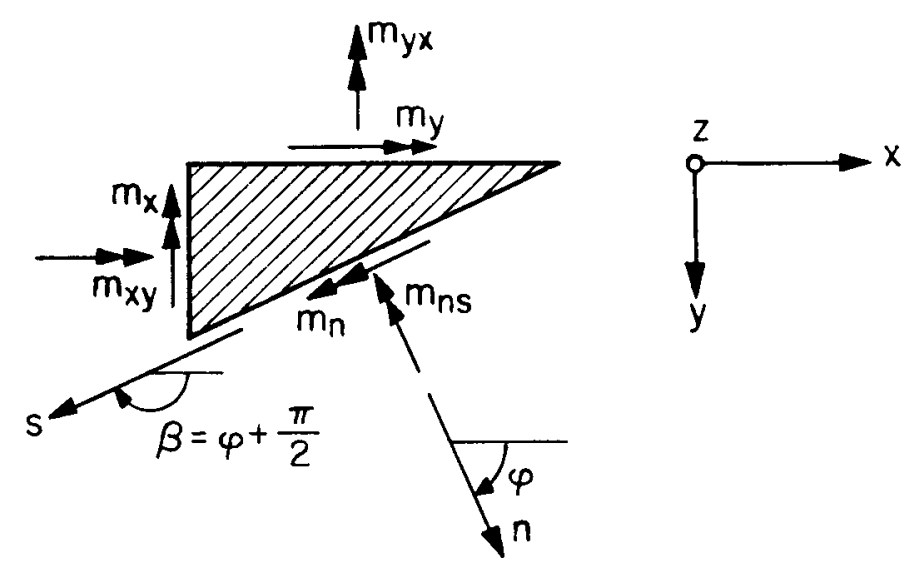

Bild 2.5: Bezeichnung der Momente

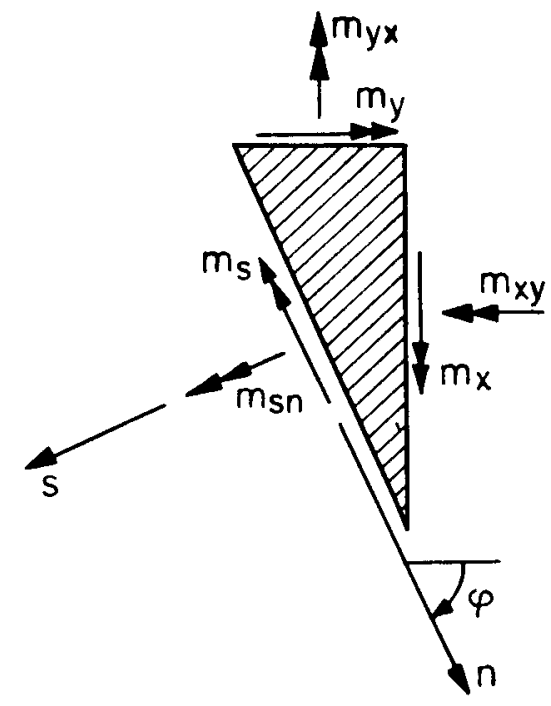

Die Beanspruchung in einer beliebigen Richtung erhält man aus Gleichgewichtsbedingungen für ein infinitesimales Dreieckelement.

$$
\begin{aligned}
& m_{n}=m_{x} \cos ^{2} \varphi+m_{y} \sin ^{2} \varphi+2 m_{x y} \sin \varphi \cos \varphi \\
& m_{s}=m_{x} \sin ^{2} \varphi+m_{y} \cos ^{2} \varphi-2 m_{x y} \sin \varphi \cos \varphi \\
& m_{n s}=-m_{x} \sin \varphi \cos \varphi+m_{y} \sin \varphi \cos \varphi+m_{x y}\left(\cos ^{2} \varphi-\sin ^{2} \varphi\right) \\
& \left(m_{s n}=m_{n s}\right)
\end{aligned}
$$

Auf dieselbe Weise lassen sich die plastischen Widerstände für diese Richtung bestimmen, z.B.

$$
P_{n}=P_{x} \cos ^{2} \varphi+P_{y} \sin ^{2} \varphi+2 P_{x y} \sin \varphi \cos \varphi
$$

Setzt man die Gleichungen (2.5) und (2.6) in (2.4) ein, bekommt man nach kurzer Umformung

$$
F(\varphi)=\left(P_{x}-m_{x}\right) \cos ^{2} \varphi+\left(P_{y}-m_{y}\right) \sin ^{2} \varphi+\left(P_{x y}-m_{x y}\right) \cdot 2 \cdot \sin \varphi \cos \varphi \geqq 0
$$

Die Funktion $F(\varphi)$ zeigt an, wie gross die "Reserve" bezüglich fliessen bei einer gewissen Beanspruchung noch ist. Gesucht sei nun derjenige Winkel $\varphi$, bei dem das Fliessen zuerst eintritt. Man kann sich auf das geschlossene 
Intervall $0 \leqq \varphi \leqq \frac{\pi}{2}$ beschränken. Die übrigen Bereiche von $\varphi$ lassen sich analog untersuchen. Es gibt drei Fälle:

(a) Randwert $\varphi=0$ :

$$
F(\phi)=0, \text { folls } \quad m_{x}=P_{x}
$$

(b) Randwert $\varphi=\frac{\pi}{2}$ :

$$
F(\varphi)=0, \text { folls } \quad m_{y}=P_{y}
$$

(c) Offenes Intervali $0<\varphi<\frac{\pi}{2}$ :

Es sind $\cos \varphi>0$ und $\sin \varphi>0$ und die Funktion

$$
G(\varphi)=\left(P_{x}-m_{x}\right) \frac{1}{\tan \varphi}+\left(P_{y}-m_{y}\right) \tan \varphi+2 \cdot\left(P_{x y}-m_{x y}\right)
$$

hat das Minimum bei $\frac{\partial G(\varphi)}{\partial \varphi}=0$, woraus:

$$
\tan ^{2} \varphi_{(\min )}=\frac{P_{x}-m_{x}}{P_{y}-m_{y}}
$$

Durch Einsetzen erhält man

$$
2 \sqrt{\left(P_{x}-m_{x}\right)} \sqrt{\left(P_{y}-m_{y}\right)}=-2 \cdot\left(P_{x y}-m_{x y}\right)
$$

und schliesslich die gesuchte Fliessbedingung für den Fall (c):

$$
\left(P_{x}-m_{x}\right)\left(P_{y}-m_{y}\right) \geqq\left(P_{x y}-m_{x y}\right)^{2}
$$

Eine Untersuchung aller möglichen Fälle für positive und negative Momente zeigt [1], dass der zulässige Bereich für die Beanspruchung durch die folgenden sechs nichtlinearen Ungleichungen gegeben ist:

$$
\begin{aligned}
P_{x}-m_{x} & \geqq 0 \\
P_{y}-m_{y} & \geqq 0 \\
\left(P_{x}-m_{x}\right)\left(P_{y}-m_{y}\right) & \geqq\left(P_{x y}-m_{x y}\right)^{2} \\
N_{x}+m_{x} & \geqq 0 \\
N_{y}+m_{y} & \geqq 0 \\
\left(N_{x}+m_{x}\right)\left(N_{y}+m_{y}\right) & \geqq\left(N_{x y}-m_{x y}\right)^{2}
\end{aligned}
$$


Das Traglastproblem soll mit den Methoden der linearen Programmierung gelöst werden. Die Bedingungen (2.13) kännen nach Wolfensberger [1] so linearisiert werden, dass die Näherung auf der sicheren Seite liegt. Man kommt auf die folgenden acht Ungleichungen:

$$
\begin{aligned}
& P_{x}+P_{x y}-m_{x}-m_{x y} \geqq 0 \\
& P_{x}-P_{x y}-m_{x}+m_{x y} \geqq 0 \\
& P_{y}+P_{x y}-m_{y}-m_{x y} \geqq 0 \\
& P_{y}-P_{x y}-m_{y}+m_{x y} \geqq 0 \\
& N_{x}+N_{x y}+m_{x}-m_{x y} \geqq 0 \\
& N_{x}-N_{x y}+m_{x}+m_{x y} \geqq 0 \\
& N_{y}+N_{x y}+m_{y}-m_{x y} \geqq 0 \\
& N_{y}-N_{x y}+m_{y}+m_{x y} \geqq 0
\end{aligned}
$$

Das Bild 2.6 zeigt eine allgemeine linearisierte Fliessfigur im Koordinatensystem $\left(m_{x}-m_{y}-m_{x y}\right)$.

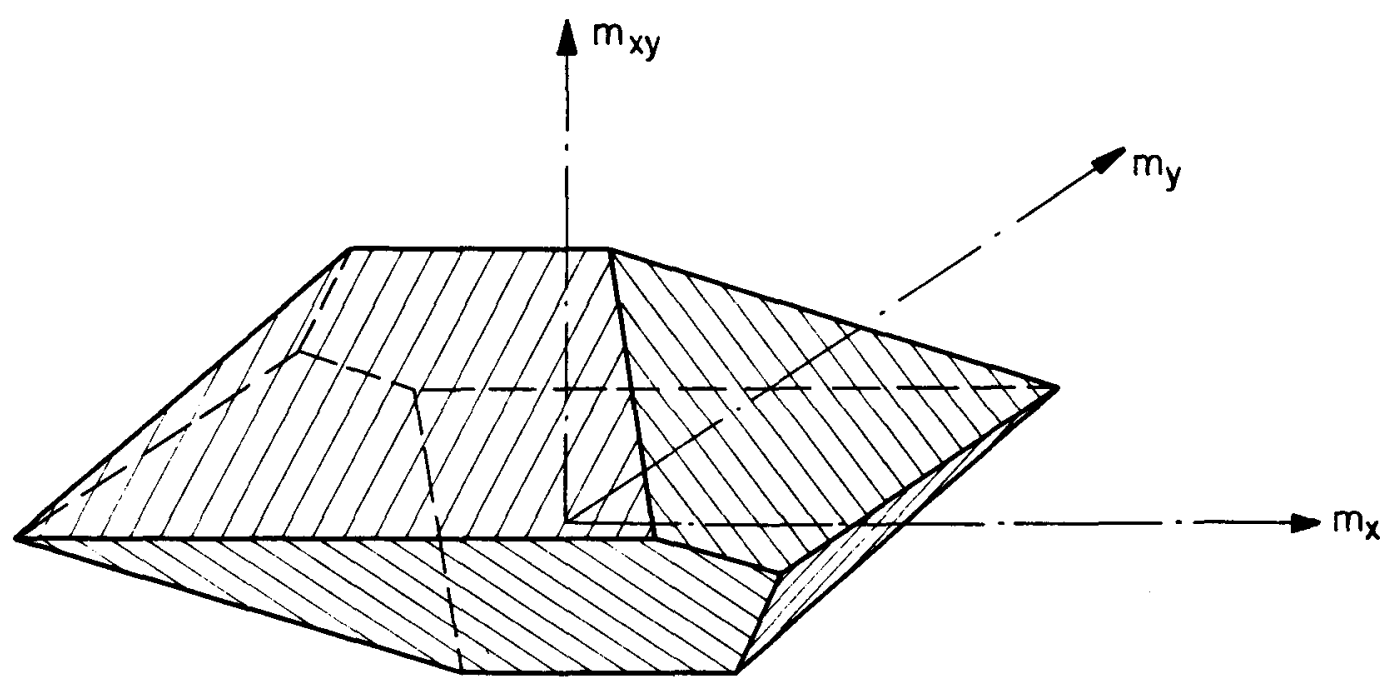

\section{Bild 2.6: Lineorisierte Fliessbedingung}

In Matrixschreibweise lautet die linearisierte Fliessbedingung für einen Punkt q der Stahlbetonplatte:

$$
\left\{c^{q}\right\}_{8 \times 1}+\left[u^{q}\right]_{8 \times 3}\left\{m_{x-y}^{q}\right\}_{3 \times 1} \geqq\{0\}
$$


Die Matrizen und Vektoren in der Gleichung (2.15) sind wie folgt definiert:

$$
\left[U^{q}\right]=\left[\begin{array}{rrr}
-1 & 0 & -1 \\
-1 & 0 & 1 \\
0 & -1 & -1 \\
0 & -1 & 1 \\
1 & 0 & -1 \\
1 & 0 & 1 \\
0 & 1 & -1 \\
0 & 1 & 1
\end{array}\right] \quad\left\{c^{q}\right\}=\left\{\begin{array}{l}
P_{x}+P_{x y} \\
P_{x}-P_{x y} \\
P_{y}+P_{x y} \\
P_{y}-P_{x y} \\
N_{x}+N_{x y} \\
N_{x}-N_{x y} \\
N_{y}+N_{x y} \\
N_{y}-N_{x y}
\end{array}\right\} \quad\left\{m_{x-y}^{q}\right\}=\left\{\begin{array}{l}
m_{x}^{q} \\
m_{y}^{q} \\
m_{x y}^{q}
\end{array}\right\}
$$

Die Matrix $\left[U^{q}\right]$ wird als Ungleichungsmatrix für den Punkt $q(x, y)$ bezeichnet.

\subsection{Besonderheiten}

Das Optimierungsverfahren (Kapitel 6) wird wesentlich vereinfacht, wenn $\{c\} \geqq\{0\}$. Aus physikalischen Gründen ist klar, dass diese Bedingung erfüllt sein muss, weil sonst die unbelastete Platte $(m \equiv 0)$ eine Fliessbedingung verletzen würde. Durch die Linearisierung geht aber diese Eigenschaft verloren. Es stellt sich z.B. die Frage, ob

$$
\begin{gathered}
c_{4}=P_{y}-P_{x y}=P_{1} \sin ^{2} \alpha+P_{2} \cos ^{2} \alpha-\left(P_{1}-P_{2}\right) \sin \alpha \cos \alpha<0 ? \\
0<\alpha<90^{\circ}:-P_{1}+P_{2}+P_{1} \tan \alpha+P_{2} \cot \alpha<0 ?
\end{gathered}
$$

Die Grenze ist also angegeben durch

$$
\frac{P_{2}}{P_{1}}=\frac{1-\tan \alpha}{1+\cot \alpha}
$$




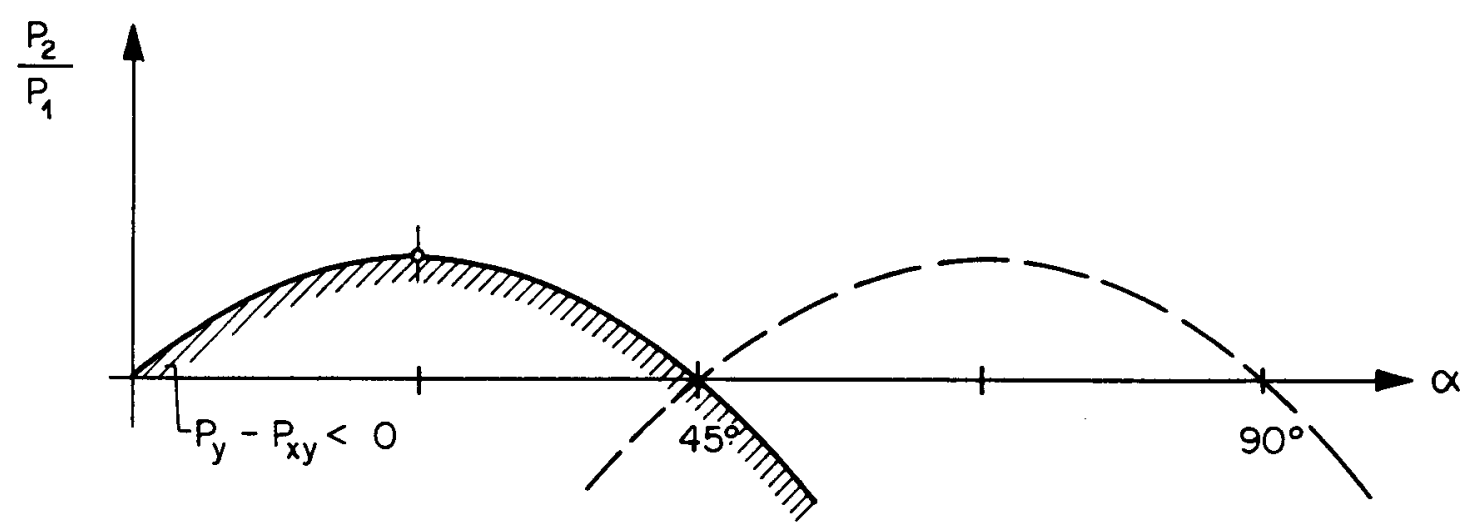

Bild 2.7: Zulässige Lösung für die unbelostete Platte

Das Maximum von $P_{2} / P_{1}$ liegt bei $\alpha=22,5^{0}$ und beträgt $(3+2 \sqrt{2})^{-1}$. Also kann $c_{4}<0$ werden, wenn $P_{2}<0,1716 P_{1}$. Die Grenzen für die übrigen Plastizitätsbedingungen führen auf den gleichen Wert. Alle Konstanten $\mathrm{c}_{i}$ werden somit für alle a positiv, wenn das Verhältnis der plastischen Hauptmomente die Bedingung $0,1716 \leqq P_{2} / P_{1} \leqq 5,8284$ erfüllt.

Dasselbe Resultat erhält man, wenn man die Beziehung (2.1) mittels des Mohr'schen Kreises darstellt.

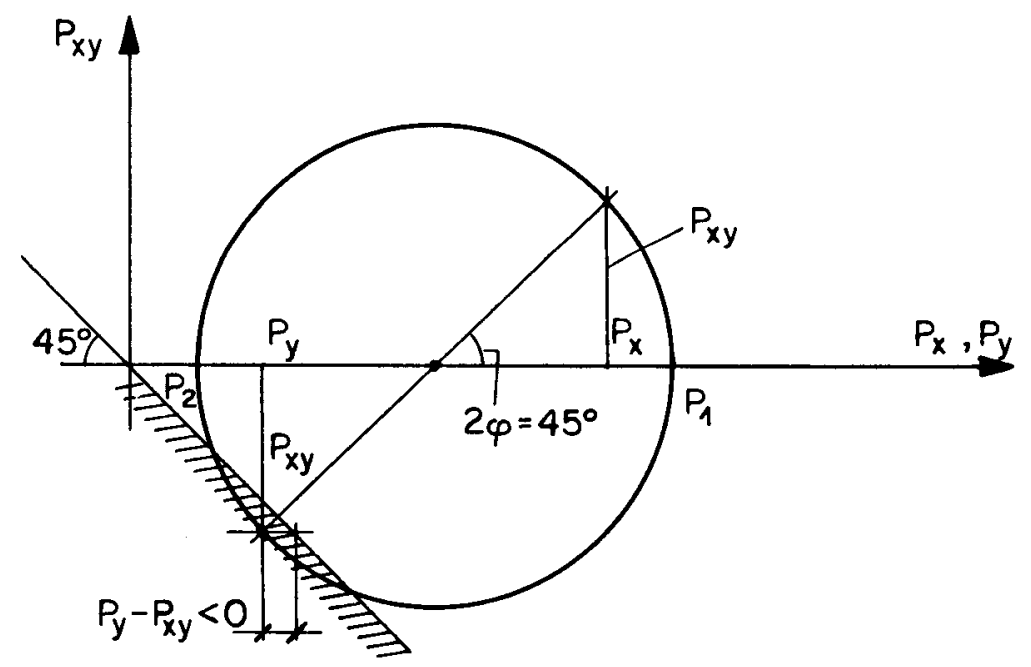

Bild 2.8: Darstellung mit dem Mohr'schen Kreis

Verwendet man die nichtlinearen Fliessbedingungen (2.13), so ist tatsächlich der Nullpunkt ( $m \equiv 0)$ in jedem Fall zulässig, weil aus dem Mohr'schen Kreis leicht ersichtlich ist, dass

$$
P_{x} P_{y} \geqq P_{x y}^{2} \quad\left(P_{1}, P_{2} \geqq 0\right)
$$


Ein Beispiel des Schnittes der Fliessfigur mit der $m_{x}{ }^{-m_{x y}}$-Ebene ist im Bild 2.9 dargestellt.

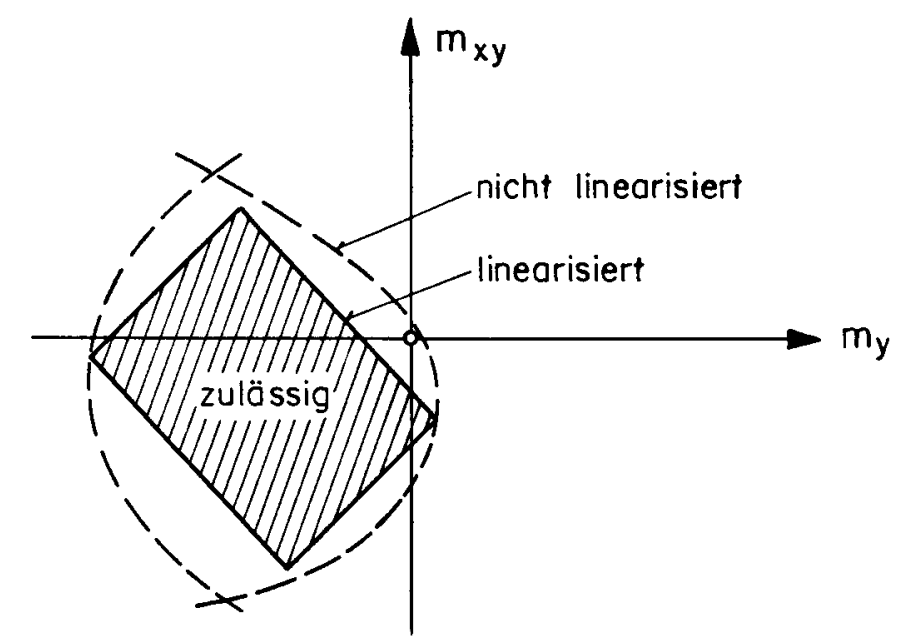

Bild 2.9: Einfluss der Linearisierung

Bei den praktischen Anwendungen ist normalerweise $P_{2}>0,1716 P_{1}$, so dass für die Optimierung vorausgesetzt werden kann, der Momenten-Nullpunkt sei zulässig, ohne die Benützbarkeit der Methode zu gefährden. Ausserdem können die Fliessbedingungen in den meisten Fällen in den Hauptrichtungen der Armierung kontrolliert werden, wamit das Problem hinfällig wird, weil $P_{12}=0$ ist.

Eine weitere Eigenschaft, die durch die Linearisierung verloren geht, ist die Invarianz der Fliessbedingung. Die Resultate sind also abhängig von der Richtung, in welcher die Kontrolle vorgenommen wird. Im Bild 2.10 liegt die orthogonale Armierung mit den positiven plastischen Momenten $P_{n}$, $P_{s} ; P_{n s}=0$ in einem Winkel $\gamma$ zu den Hauptaxen $(1-2)$ der Beanspruchung $m_{1}, m_{2} ; m_{12}=0$.

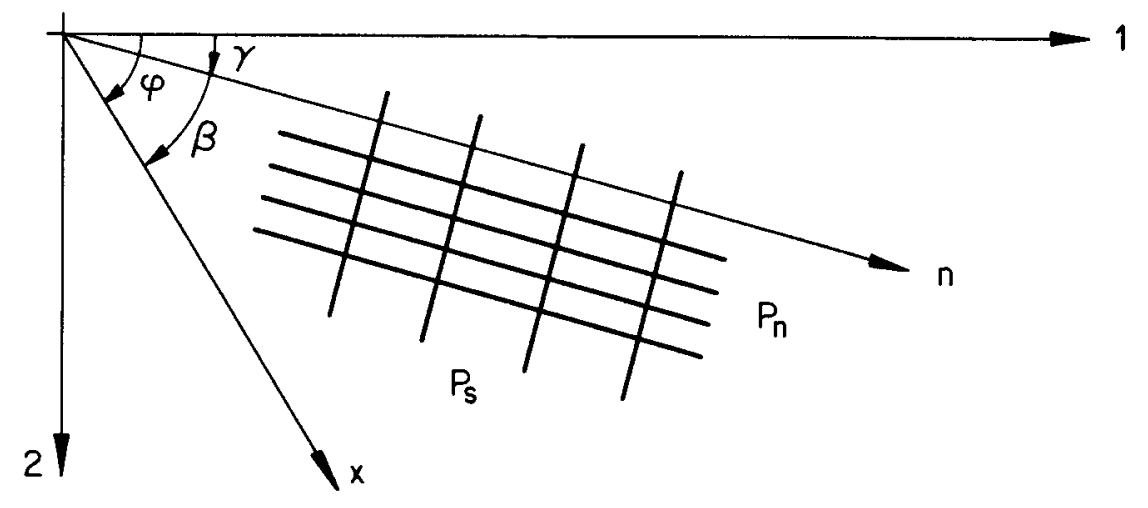

Bild 2.10: Houptoxen und Kontrollrichtung 
Zuerst soll bewiesen werden, dass die nichtlineare Fliessbedingung (2.13) von der Richtung $x$, in welcher die Kontrolle durchgeführt wird, unabhängig ist. Die positiven Drillungsmomente werden durch die Bedingung

$$
\left\langle P_{x}-m_{x}\right)\left(P_{y}-m_{y}\right) \geqq\left(P_{x y}-m_{x y}\right)^{2}
$$

begrenzt. Die Bedingungen für negative Beanspruchungen werden nicht untersucht, weil das analoge Resultat ohne weiteres ersichtlich ist. Man setzt die Widerstände und Beanspruchungen im Koordinatensystem $(x-y)$

$$
\begin{aligned}
& P_{x}=P_{n} \cos ^{2} \beta+P_{s} \sin ^{2} \beta \\
& P_{y}=P_{n} \sin ^{2} \beta+P_{s} \cos ^{2} \beta \\
& P_{x y}=\left(P_{s}-P_{n}\right) \sin \beta \cos \beta \\
& m_{x}=m_{1} \cos ^{2} \varphi+m_{2} \sin ^{2} \varphi \\
& m_{y}=m_{1} \sin ^{2} \varphi+m_{2} \cos ^{2} \varphi \\
& m_{x y}=\left(m_{2}-m_{1}\right) \sin \varphi \cos \varphi
\end{aligned}
$$

$\left(P_{n} \cos ^{2} \beta+P_{s} \sin ^{2} \beta-m_{1} \cos ^{2} \varphi-m_{2} \sin ^{2} \varphi\right)\left(P_{n} \sin ^{2} \beta+P_{s} \cos ^{2} \beta-m_{1} \sin ^{2} \beta-m_{2} \cos ^{2} \beta\right)$ $\geqq\left[\left(P_{s}-P_{n}\right) \sin \beta \cos \beta-\left(m_{2}-m_{1}\right) \sin \varphi \cos \varphi\right]^{2}$

Die Ausrechnung dieses Ausdruckes führt auf die Bedingung

$$
P_{n} P_{s}+m_{1} m_{2}-\left(P_{n} m_{1}+P_{s} m_{2}\right) \sin ^{2}(\varphi-\beta)-\left(P_{n} m_{2}+P_{s} m_{1}\right) \cos ^{2}(\varphi-\beta) \geqq 0
$$

Man berücksichtigt noch, dass $\beta=\varphi-\gamma$ und bekommt die Ungleichung

$$
P_{n} P_{s}+m_{1} m_{2} \geqq\left(P_{n} m_{1}+P_{s} m_{2}\right) \sin ^{2} \gamma+\left(P_{n} m_{2}+P_{s} m_{1}\right) \cos ^{2} \gamma
$$


Die Biegemomente werden noch durch die Bedingungen

$$
\begin{aligned}
& P_{x}-m_{x}=P_{n} \cos ^{2} \beta+P_{s} \sin ^{2} \beta-m_{1} \cos ^{2} \varphi-m_{2} \sin ^{2} \varphi \geqq 0 \\
& P_{y}-m_{y}=P_{n} \sin ^{2} \beta+P_{s} \cos ^{2} \beta-m_{1} \sin ^{2} \varphi-m_{2} \cos ^{2} \varphi \geqq 0
\end{aligned}
$$

beschränkt. Man kann diese Ungleichungen ersetzen durch

$$
\begin{aligned}
& \left(P_{x}-m_{x}\right)+\left(P_{y}-m_{y}\right) \geqq 0 \\
& \left(P_{x}-m_{x}\right) \cdot\left(P_{y}-m_{y}\right) \geqq 0
\end{aligned}
$$

Die erste Bedingung führt auf die von $\varphi$ unabhängige Beschränkung

$$
P_{n}+P_{s}-m_{1}-m_{2} \geqq 0
$$

Die zweite Bedingung ist automatisch für jedes $\varphi$ erfüllt, wenn (2.18) für jedes $\varphi$ erfüllt ist.

Um zu beweisen, dass die linearisierten Fliessbedingungen nicht mehr invariant sind, genügt es, ein Beispiel zu finden, bei dem die Kontrolle in zwei verschiedenen Richtungen nicht dasselbe Resultat ergibt. Das ist z.B. bei den folgenden Zahlenwerten für die plastischen Widerstände und Beanspruchungen der fall:

$$
\begin{array}{lll}
P_{n}=3,70 & m_{1}=3,0 & \\
P_{s}=8,00 & m_{2}=4,0 & \gamma=\frac{\pi}{6} \\
P_{n s}=0 & m_{12}=0 &
\end{array}
$$

Daraus ergeben sich

$$
\begin{aligned}
& P_{(1)}=3,70 \cdot 0,75+8,0 \cdot 0,25=4,775 \\
& P_{(2)}=3,70 \cdot 0,25+8,0 \cdot 0,75=6,925 \\
& P_{(12)}=-(8,0-3,70) \cdot 0,25 \cdot \sqrt{3}=-1,862 \\
& m_{n}=3,0 \cdot 0,75+4,0 \cdot 0,25=3,25 \\
& m_{s}=3,0 \cdot 0,25+4,0 \cdot 0,75=3,75 \\
& m_{n s}=(4,0-3,0) \cdot 0,25 \cdot \sqrt{3}=0,433
\end{aligned}
$$


Die Kontrolle der positiven Momente in der Richtung 1

$$
\begin{aligned}
& 2,913=P_{(1)}+P_{(12)} \leqq m_{1}=3,0 ! \\
& 6,637=P_{(1)}-P_{(12)} \geqq m_{1}=3,0 \\
& 5,063=P_{(2)}+P_{(12)} \geqq m_{2}=4,0 \\
& 8,778=P_{(2)}-P_{(12)} \geqq m_{2}=4,0
\end{aligned}
$$

ergibt, dass die linearisierte Fliessbedingung nicht erfüllt ist. Demgegenüber resultiert aus der Kontrolle für die Richtung $n$,

$$
\begin{aligned}
& 3,70=P_{n} \geqq m_{n}+m_{n s}=3,683 \\
& 3,70=P_{n} \geqq m_{n}-m_{n s}=2,817 \\
& 8,0=P_{s} \geqq m_{s}+m_{n s}=4,183 \\
& 8,0=P_{s} \geqq m_{s}-m_{n s}=3,317
\end{aligned}
$$

dass die linearisierte Fliessbedingung erfüllt ist. Die Fliessbedingung (2.14) ist also nicht mehr invariant. 
3.1 Einführung der Methode der finiten Elemente

Für die Theorie der dünnen Platten mit kleiner Durchbiegung gelten die folgenden Voraussetzungen [11]. Die Punkte auf einer Normalen zur Mittelebene bleiben auf dieser Normalen (keine Schubverformungen) und die Verformungen der Mittelebene werden vernachlässigt (kein Membraneinfluss). Diese Voraussetzungen für den Verformungszustand der Platte sind im Bild 3.1 dargestellt.

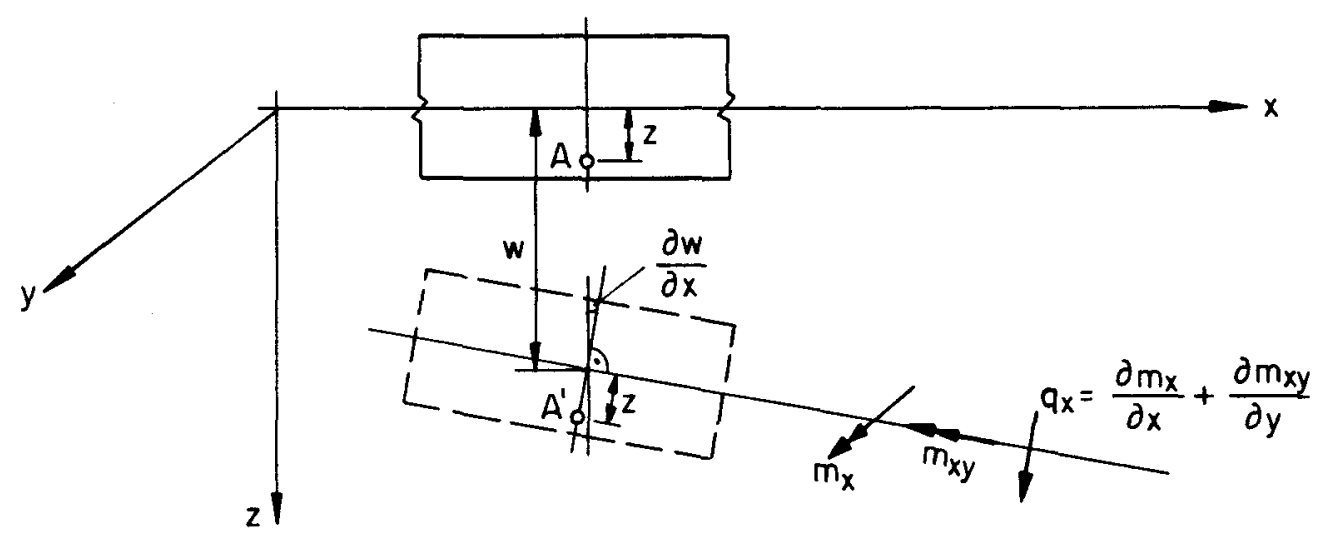

Bild 3.1: Verformungs - und Spannungszustand der Platte

Der Verformungszustand kann also durch eine einzige Funktion w(x,y) für die vertikalen Verschiebungen beschrieben werden. Der Spannungszustand ist durch die drei unabhängigen Funktionen für die Spannungsresultierenden $m_{x}(x, y)$, $m_{y}(x, y)$ und $m_{x y}(x, y)$ beschrieben. Als Belastung werden hier nur vertikale Lasten $\lambda \cdot p(x, y)$ betrachtet. Der Faktor $\lambda$ bringt zum Ausdruck, dass für die Berechnung der Traglast die gesamte äussere Last proportional bis zum Kollaps erhöht wird. Er ist das Verhältnis der Kollapslast zur Belastung $p(x, y)$.

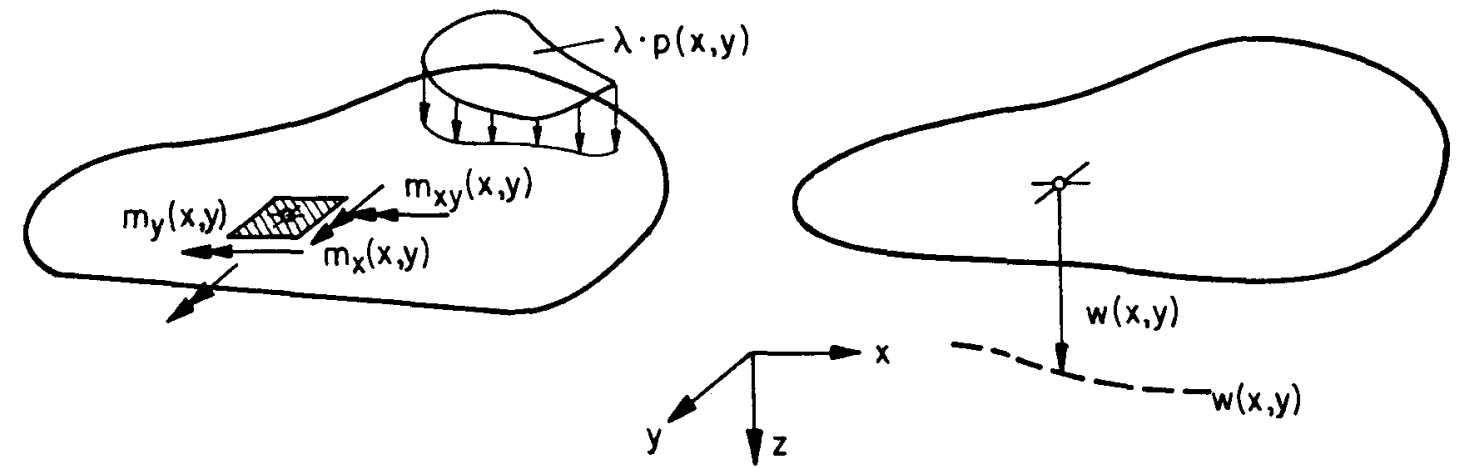

Bild 3.2: Momente, Belastung und Verschiebungen 
Aus den Verschiebungen $w(x, y)$ ergeben sich unter Benützung der obenstehenden Voraussetzungen die Krümmungen $\kappa_{x}(x, y)=-w, x x$, $\kappa_{y}=-w$, yy und $\kappa_{x y}=$ $-w, x y^{*}$ Der Begriff der Krümmung wird hier im verallgemeinerten Sinn benutzt, d.h. die Verwindung $k_{x y}$ wird auch darunter subsumiert.

Der Traglastfaktor $\lambda$ kann aufgrund der zwei Grenzwertsätze der Plastizitätstheorie ermittelt werden [3].

Statischer Grenzwertsatz (Satz über den untern Grenzwert): Gesucht ist ein möglichst grosser Wert des Traglastfaktors $\lambda$. Die Belastung $\lambda \cdot p(x, y)$ muss mit den Momenten $m_{i j}(x, y),(i=x, y ; j=x, y)$ im Gleichgewicht stehen. Die Momente $m_{i j}(x, y)$ dürfen die Plastizitätsbedingungen nirgends verletzen.

Kinematischer Grenzwertsatz (Satz über den obern Grenzwert): Gesucht ist ein möglichst kleiner Wert des Traglastfaktors $\lambda$. Im Kollapszustand ist die Leistung $L$ der Belastung $\lambda \cdot p(x, y)$ gleich der Dissipationsleistung D. Die zur Bestimmung von $L$ verwendeten Verschiebungsgeschwindigkeiten $\dot{w}(x, y)$ müssen mit den zur Bestimmung von $D$ verwendeten Krümmungsgeschwindigkeiten $\dot{k}(x, y)$ kinematisch verträglich sein.

Wenn diese Bedingungen erfüllt sind, wird $m_{i j}(x, y)$ als ein optimaler, stabiler, statisch zulässiger Spannungszustand resp. $\dot{w}(x, y)$ als ein optimaler, instabiler, kinematisch zulässiger Bewegungszustand (optimaler Mechanismus) bezeichnet. Die zugehörige Traglast ist dann ein unterer resp. ein oberer Grenzwert der wirklichen Traglast.

Die Auffindung der exakten Funktionen für $\mathrm{m}_{i j}(x, y)$ und $w(x, y)$ ist nur in wenigen Spezialfällen möglich. Für die numerische Traglastberechnung beliebiger Platten ist die Anwendung der Methode der finiten Elemente zweckmässig. Die Funktionen $m_{i j}$ und $w$ werden in der Regel durch Polynome im Bereich der Elemente approximiert.

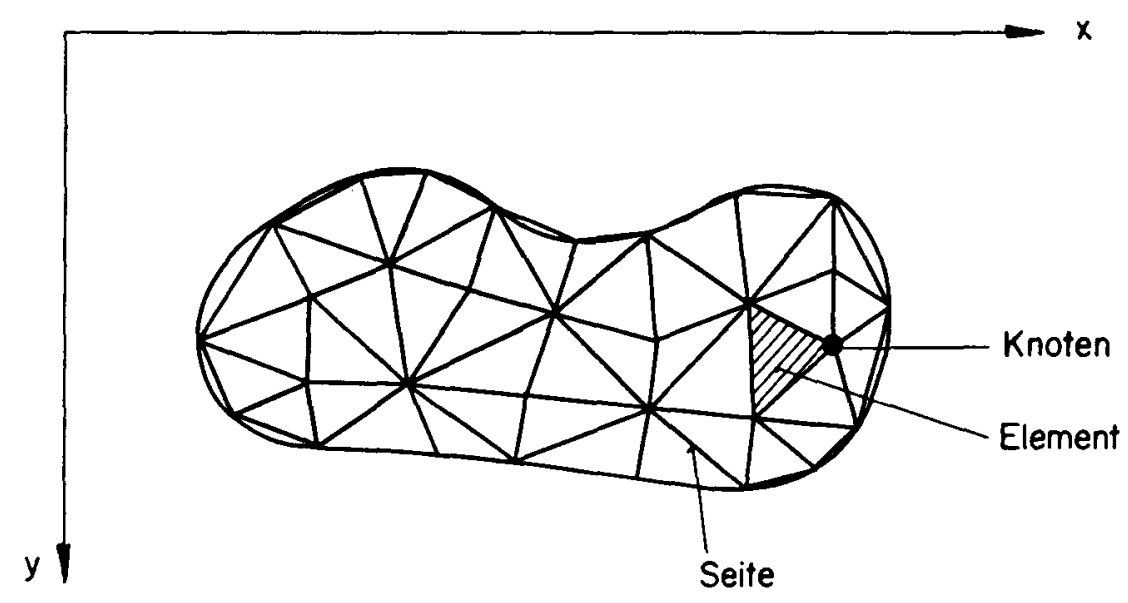


Als Parameter bezeichnet man einen Satz von Stützwerten, durch den - zusammen mit dem gewählten Verlauf innerhalb der Elemente - der wert der betreffenden Funktion an jeder Stelle der Platte definiert ist. Bei der Methode der finiten Elemente sind die Parameter (im Gegensatz zum allgemeinen Ritzschen Verfahren und der Approximation durch Fourier-Reihen) direkt mit anschaulichen physikalischen Grössen verbunden.

Die vertikalen Verschiebungen und die Momente werden durch folgende Funktionen approximiert:

$$
\begin{array}{rlrl}
w(x, y) & =\varphi_{m}(x, y) w_{m} & & (m=1, M) \\
m_{i j}(x, y) & =\psi_{i j r}(x, y) m_{r} & (r=1, R)
\end{array}
$$

Innerhalb eines Elementes e ist der Verlauf von $w$ und $m_{i j}$ gegeben durch die sogenannten Interpolationsfunktionen $\varphi_{k}^{e}$ und $\psi_{i j l}^{e}$ und die mit diesem Element verbundenen Parameter.

$$
\begin{aligned}
& w(x, y)=\varphi_{k}^{e}(x, y) w_{k}^{e} \quad\left(k=1, K^{e}\right) \\
& m_{i j}(x, y)=\psi_{i j l}^{e}(x, y) m_{i j l}^{e} \quad\left(L=1, L^{e}\right)
\end{aligned}
$$

Im Bild 3.4 ist ein einfaches Beispiel von Interpolationsfunktionen und Parametern für die vertikalen Verschiebungen $w(x)$ eines stabelementes dargestelit.

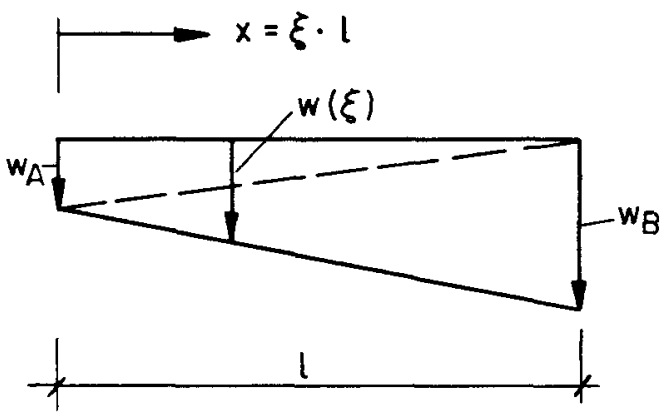

$$
\begin{aligned}
& \text { Verlauf linear } \\
& \begin{aligned}
w(\xi) & =d_{1}(1-\xi)+d_{2} \cdot \xi \\
d_{1} & =w_{A}, d_{2}=w_{B}
\end{aligned}
\end{aligned}
$$

\section{Bild 3.4: Porameter und Näherungsfunktion für ein Stabelement}


Die vertikalen Verschiebungen des Stabelementes werden also approximiert durch

$$
w(\xi)=\sum_{k=1}^{2} \varphi_{k}^{e}(\xi) \cdot d_{k}=\varphi_{k}^{e} d_{k}
$$

wobei $\varphi_{k}^{e}(\xi)$ als Interpolationsfunktionen und $d_{k}$ als Parameter bezeichnet werden. Die Parameter sind direkt mit anschaulichen physikalischen Grössen verbunden.

Die lokalen Parameter eines Plattenelementes, $w_{k}^{e}$ und $m_{i j l}^{e}$, sind durch einfache topologische Beziehungen mit den globalen Parametern der Platte verknüpft:

$$
\begin{aligned}
& w_{k}^{e}=a_{k m}^{e} w_{m} \\
& m_{i j l}^{e}=b_{l r}^{e} m_{r}
\end{aligned}
$$

Dabei ist

$$
\begin{array}{lllll}
a_{k m}^{e}=1 & \text { falls } & w_{k}^{e}=w_{m} & \text { sonst } & a_{k m}^{e}=0 \\
b_{l r}^{e}=1 & \text { folls } & m_{i j l}^{e}=m_{r} & \text { sonst } & b_{l r}^{e}=0
\end{array}
$$

Im Bereich des Elementes e ist also

$$
\begin{aligned}
& \varphi_{m}=a_{k m}^{e} \varphi_{m}^{e} \\
& \psi_{i j r}=b_{l r}^{e} \psi_{i j l}^{e}
\end{aligned}
$$

Die im statischen und kinematischen Grenzwertsatz enthaltenen Bedingungen müssen jetzt für die Näherungsfunktionen (3.1) und (3.2) formuliert werden. Vorerst wird nur der statische Grenzwertsatz betrachtet; der kinematische Grenzwertsatz wird im Kapitel 6 behandelt.

Die Gleichgewichtsbedingungen für Platten sind erfüllt, wenn für jede virtuelle Verschiebung $w^{*}(x, y)$, welche die kinematischen Randbedingungen erfüllt,

$$
\iint_{F} K_{i j}^{*}(x, y) m_{i j}(x, y) d F-\lambda \iint_{F} w^{*}(x, y) p(x, y) d F=0
$$

ist. Die später verwendete Dualität (Kapitel 6) der Gleichungen und Ungleichungen, die sich aus den Grenzwertsätzen ergeben, bedingt, dass sich die Parameter und der Verlauf der virtuellen Verschiebungen, die für die Gleich- 
gewichtskantrollen verwendet werden, und diejenigen für den Mechanismus entsprechen:

$$
\kappa_{i j}^{*}=-w_{, i j}^{*}=-w_{m}^{*} \varphi_{m, i j}
$$

Das Einsetzen der Näherungsfunktionen liefert

$$
w_{m}^{*}\left(\left[-\iint_{F} \varphi_{m, i j} \psi_{i j r} d F\right] m_{r}-\lambda \iint_{F} \varphi_{m} p d F\right)=0
$$

Man erhält für jede unabhängige Variation eines Verschiebungsparameters eine Gleichgewichtsgleichung, z.B.

$$
g_{m r} m_{r}-\lambda p_{m}=0
$$

wobei

$$
\begin{aligned}
& g_{m r}=-\iint_{F} \varphi_{m, i j} \psi_{i j r} d F \\
& p_{m}=\iint_{F} \varphi_{m} p d F
\end{aligned}
$$

Der Koeffizient $g_{m r}$ ist die innere Arbeit der Momente infolge $m_{r}=1$ für die Krümmungen infolge $w_{m}^{*}=1$; der Koeffizient $P_{m}$ ist die Arbeit der äusseren Lasten $p(x, y)$ für die Verschiebungen infolge $w_{m}^{*}=1$.

Insgesamt ergibt sich ein System von M Gleichungen, das in Matrixschreibweise wie folgt geschrieben werden kann:

$$
[G]\{m\}-\lambda \cdot\{p\}=\{0\}
$$

Die in der Gleichung (3.2) definierten Approximationsfunktionen für $m_{i j}(x, y)$ müssen auch die Plastizitätsbedingungen erfüllen. Die Plastizitätsbedingungen sollen in $Q$ Kontrollpunkten mit den Koordinaten $x^{q}, y^{q}(q=1$, Q) kontrolliert werden. An einem Kontrollpunkt $q$ ist nach Gleichung (3.2)

$$
m_{i j}^{q}=m_{i j}\left(x^{q}, y^{q}\right)=\psi_{i j r}\left(x^{q}, y^{q}\right) m_{r}=\psi_{i j r}^{q} m_{r}
$$

Der Vektor $m^{q}$ hat die drei komponenten $m_{x}^{q}, m_{y}^{q}$ und $m_{x y}^{q}$; der Vektor $m$ hat $R$ Komponenten. In Matrixschreibweise ist 


$$
\left\{m^{q}\right\}=\left[\psi^{q}\right] \quad\{m\}
$$

Durch Einsetzen dieses Ausdruckes in die Gleichung (2.15) erhält man für jeden der $Q$ Kontrollpunkte einen Satz von acht Ungleichungen.

$$
\{0\} \leqq\left\{c^{q}\right\}+\left[U^{q}\right]\left[\psi^{q}\right]\{m\}
$$

Damit sind alle Bedingungen des statischen Grenzwertsatzes für die Näherungsfunktionen formuliert. Sie können im folgenden linearen Programm zusammengefasst werden.

$$
\begin{aligned}
\lambda & \longrightarrow \text { Maximum } \\
\{O\} & =[G]\{m\}-\{p\} \lambda \\
\{O\} \leqq & \leqq c\}+[U]\{m\}
\end{aligned}
$$

Der Vektor $\{c\}$ hat die Dimension $8 Q$ und setzt sich aus den Subvektoren $\left\{c^{q}\right\}$ $(q=1, Q)$ zusammen. Die Matrix [U] wird als Ungleichungsmatrix bezeichnet und setzt sich aus den Submatrizen $\left[U^{q}\right]\left[\psi^{q}\right](Q=1, Q)$ zusammen. Das Iineare Programm ist im Tableau 3.1 dargestelit.

Bei der Formulierung eines linearen Programmes werden die Ungleichungen im allgemeinen in Gleichungen verwandelt, indem man nichtnegative Schlupfvariable $y_{i}$ einführt. Die letzte Zeile der Gleichung (3.21) wird dann zu

$$
\begin{aligned}
& \{y\}=\{c\}+[U]\{m\} \\
& \{y\} \geqq\{0\}
\end{aligned}
$$




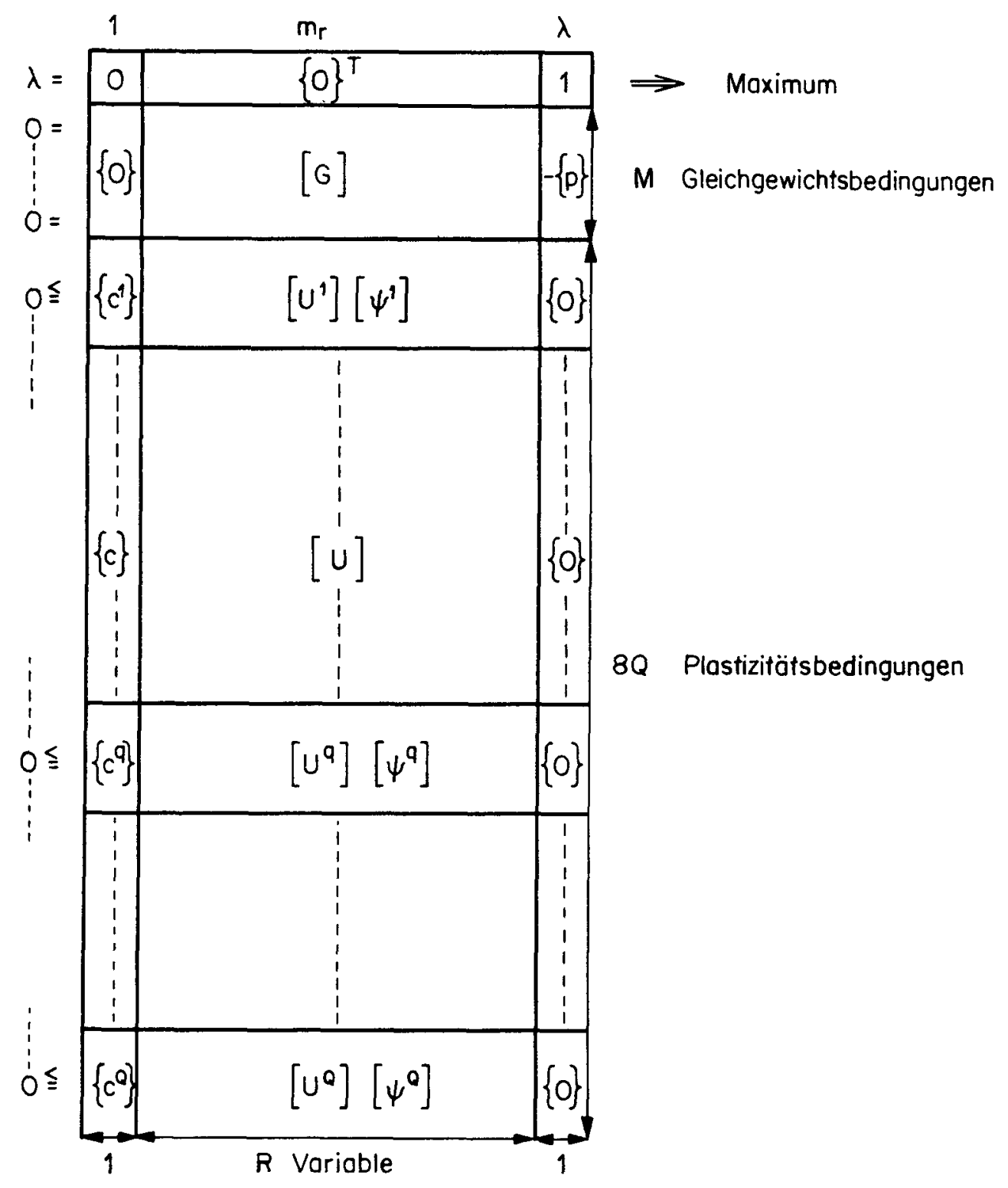

Tableau 3.1: Lineares Programm aus dem statischen Grenzwertsatz 
Die Approximationsfunktionen für den Momenten- und den Verschiebungszustand können nicht unabhängig voneinander gewählt werden. Aus den Diskontinuitäten des gewählten Verschiebungszustandes ergeben sich Kontinuitätsbedingungen für die Momente [3]. Das für die Formulierung des Gleichgewichtes in der Gleichung (3.11) verwendete Integral $\iint k_{i j}{ }^{*} m_{i j} d F(i=x, y ; j=x, y)$ und damit das Integral der Gleichung (3.15) kann nur ausgewertet werden, wenn diese Kontinuitätsbedingungen erfüllt sind. Aus dem gewählten Momentenzustand - insbesondere aus seinen Diskontinuitäten - können sodann äquivalente äussere Lasten berechnet werden.

Die Frage soll anhand eines einfachen Problems, eines Stabes mit zwei Elementen (Bild 3.5), diskutiert werden.

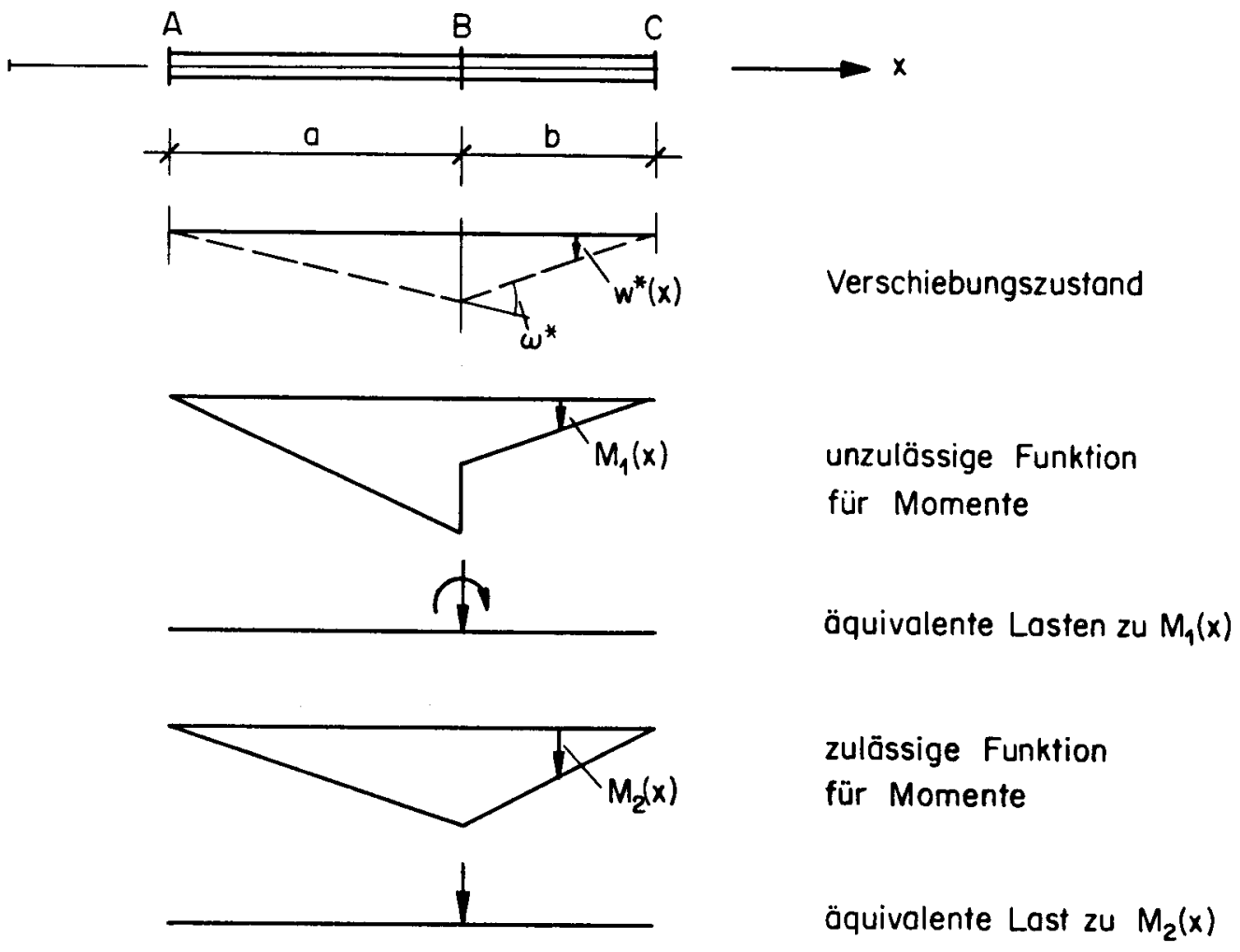

Bild 3.5: Funktionen für Verschiebung und Moment am Stabelement

Die Diskontinuität von $M_{1}(x)$ ist nicht zulässig, weil das Integral $\int M_{1}{ }^{*}{ }^{*} x x d x=M_{1 B^{\omega}}{ }^{*}$ nicht bestimmbar ist. Man sieht auch, dass der virtuelle Verschiebungszustand $w^{*}(x)$ nicht ausreicht, um das Gleichgewicht zu kontrollieren. Durch die korrekte Annahme des approximativen Verlaufes $M_{2}(x)$ ohne Sprung bei $B$ ergibt sich als äquivalente äussere Last für das Stabelement eine vertikale Einzellast. 
Eine weitere Ueberlegung bezieht sich auf das Verhältnis der Anzahl R der Parameter für den Momentenzustand zur Anzahl M der Parameter für den Verschiebungszustand. Beim Satz über den untern Grenzwert werden an die R Parameter des Momentenzustandes $M$ Gleichgewichtsbedingungen gestellt (Tableau 3.1). Damit verbleiben für die Optimierung noch R-M unabhängige Variable. Ist $M=R$, so ist das Problem statisch bestimmt. Als ideales Verhältnis für Platten wird

$$
R / M=3
$$

angenommen [3]. Dieses Verhältnis entspricht demjenigen der Anzahl unabhängiger Funktionen für Momente zur Anzahl unabhängiger Funktionen für Verschiebungen der Platte (Bild 3.1).

Schliesslich müssen die Näherungsfunktionen so gewählt werden, dass der Momentenzustand bezüglich der Plastizitätsbedingungen auf einfache Weise vollständig kontrolliert werden kann.

\subsection{Gewählte Ansätze}

Für die Formulierung des Gleichgewichtes wird die Methode der finiten Elemente benutzt, d.h. die Drtsfunktionen $m_{x}(x, y), m_{y}(x, y), m_{x y}(x, y)$ und $w(x, y)$ werden durch ein R-parametriges Momenten- resp. ein M-parametriges Verschiebungsfeld approximiert. Um eine gute Approximation der Geometrie zu erreichen, werden dreieckige Elemente gewählt (Bild 3.3). Die Anzahl Knoten wird mit NK, die Anzahl Seiten mit NS und die Anzahl Elemente mit NE bezeichnet.

Eine einfache Kontrolle der Plastizitätsbedingungen ist möglich, wenn die Momente im Element konstant oder linear verlaufen. Bei nichtlinearen Ansätzen ergeben sich beträchtliche Schwierigkeiten [12], weil der Ort der extremalen Beanspruchung an einem noch zu bestimmenden Ort innerhalb des Elementes liegen kann. Eine einfache Kontrolle des Gleichgewichtes ist möglich, indem in jedem knoten die vertikale verschiebung variiert wird. Damit ergeben sich etwa NK Verschiebungsparameter (genau genommen werden noch einige Verschiebungsparameter wegfallen, weil in einigen Knoten $w^{*}=0$ sein wird). 
Die Anzahl der Momentenparameter soll gemäss der Gleichung (3.23) etwa 3 NK sein. Das ist bei den folgenden zwei Ansätzen der Fall:

TLL Drei Momente pro Knoten als Parameterwerte, Verlauf linear über das Element [7].

TLC Ein Moment $m_{n}$ pro Seite als Parameterwert, Verlauf konstant im Element (NS

Die Abkürzung "TLL" steht für "triangular element, linear displacements, linear moments", "TLC" steht für "triangular element, linear displacements, constant moments"; $m_{n}$ ist das Biegemoment, das durch die Spannungen normal zur Seite des Elementes aufgebaut wird.

Die Randbedingungen werden nicht beachtet, weil die Gleichung (3.23) nur für eine Einteilung in sehr viele Elemente sinnvoll ist und die Anzahl der Randbedingungen in diesem Fall wesentlich kleiner als $R$ ist.

Man stellt fest, dass $m_{n}$ bei beiden Ansätzen kontinuierlich ist, d.h. einen eindeutig definierten Wert entlang den Elementrändern hat, was notwendig ist wegen der Diskontinuität von ${ }^{*}{ }^{*}, \mathrm{nn}$.

Beim Ansatz TLL genügt es, die linearisierten Plastizitätsbedingungen (2.15) in den Knoten zu kontroliieren, um sicher zu sein, dass sie in jedem Punkt der Platte erfüllt sind. Damit ergeben sich 8 NK Ungleichungen. Beim Ansatz TLC sind die (konstanten) Momente in jedem Element zu kontroliieren, d.h. es ergeben sich $8 \mathrm{NE} \cong 16$ NK Ungleichungen. 


\subsection{Ansätze und Bezeichnungen}

Die Momente und die vertikalen Verschiebungen werden mit den topologischen Bezeichnungen im Bild 4.1 definiert.

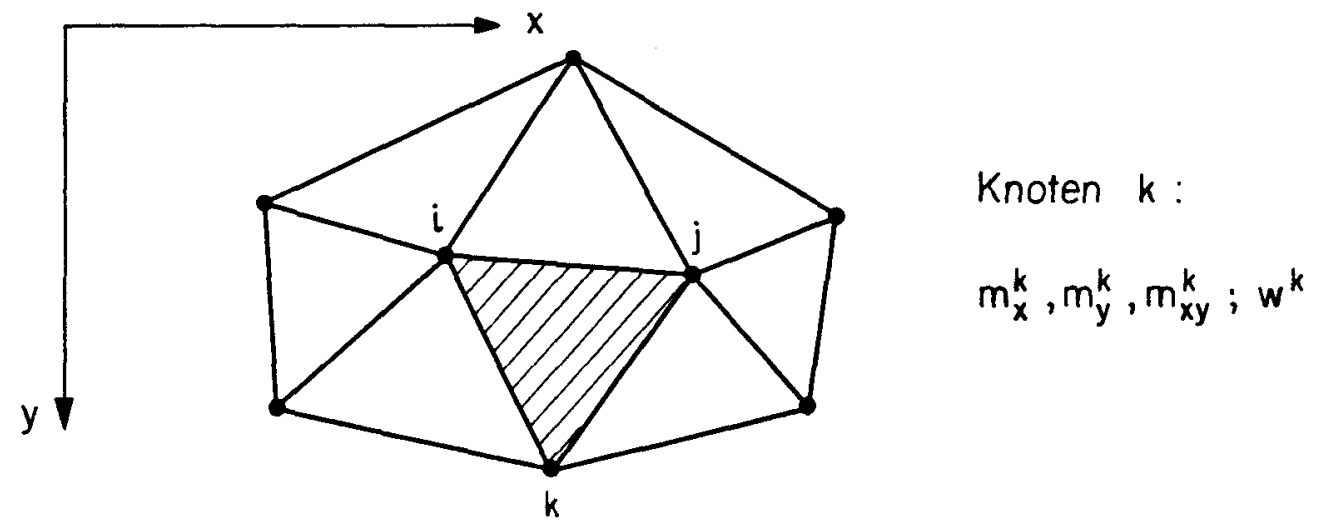

Bild 4.1 : Topologie

Die Werte der Mamente und Verschiebungen innerhalb der Elemente lassen sich wie folgt durch die Werte in den Knotenpunkten ausdrücken:

$$
\left\{\begin{array}{c}
m_{x} \\
m_{y} \\
m_{x y} \\
\cdots w
\end{array}\right\}=\left[\begin{array}{ccc}
m_{x}^{i} & m_{x}^{j} & m_{x}^{k} \\
m_{y}^{i} & m_{y}^{j} & m_{y}^{k} \\
m_{x y}^{i} & m_{x y}^{j} & m_{x y}^{k} \\
\hdashline w^{i} & w j & w^{k}
\end{array}\right] \quad\left\{\begin{array}{l}
a_{1} \\
a_{2} \\
a_{3}
\end{array}\right\}
$$

Die natürlichen Koordinaten $a_{1}, a_{2}, a_{3}$ des Dreieckes ijk stellen den linearen Verlauf der Funktionen innerhalb der Elemente auf einfache Weise dar. Sie sind im Bild 4.2 definiert. 


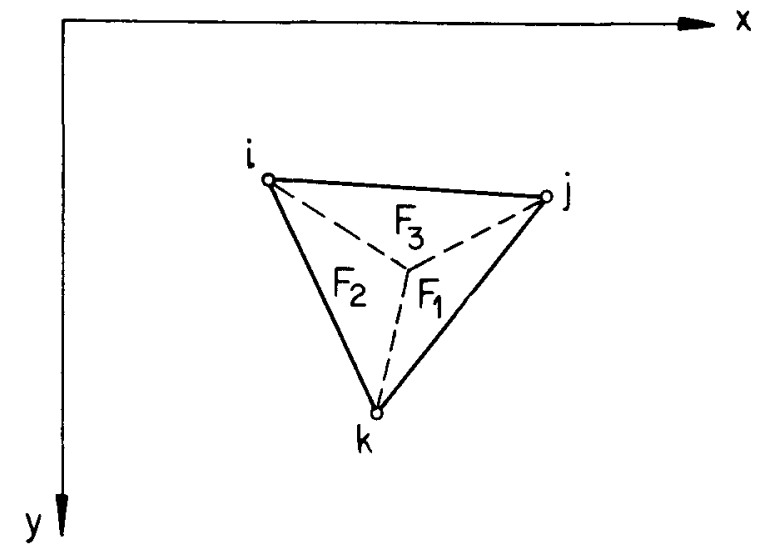

$$
\begin{aligned}
& a_{1}=F_{1} / F \\
& a_{2}=F_{2} / F \\
& a_{3}=F_{3} / F \\
& F_{1}+F_{2}+F_{3}=F \\
& \text { oder: } a_{1}+a_{2}+a_{3}=1
\end{aligned}
$$

Bild 4.2: Natürliche Koordinaten

Die Bezeichnung und die Vorzeichenregel für die Momente sind im Bild 2.4 angegeben worden. Die Transformationsformel (2.5) für die Momente in einem Punkt $P(x, y)$ soll noch in Matrixschreibweise angegeben werden:

$$
\left\{m_{n-s}^{p}\right\}=\left[T_{\varphi}\right] \quad\left\{m_{x-y}^{p}\right\}
$$

In Funktion von $\beta$ (Bild 2.5) lautet die Beziehung (2.5):

$$
\begin{aligned}
& m_{n}=m_{x} \sin ^{2} \beta+m_{y} \cos ^{2} \beta-2 m_{x y} \sin \beta \cos \beta \\
& m_{s}=m_{x} \cos ^{2} \beta+m_{y} \sin ^{2} \beta+2 m_{x y} \sin \beta \cos \beta \\
& m_{n s}=m_{x} \sin \beta \cos \beta-m_{y} \sin \beta \cos \beta-m_{x y}\left(\cos ^{2} \beta-\sin ^{2} \beta\right) \\
& \text { oder: }\left\{m_{n-s}^{P}\right\}=\left[T_{\beta}\right]\left\{m_{x-y}^{P}\right\}
\end{aligned}
$$

\subsection{Gleichgewichtsbedingungen}

Die allgemeine Formulierung des Gleichgewichtes wurde im letzten Kapitel hergeleitet. Bei der mathematischen Behandlung des Problems ergeben sich insofern Schwierigkeiten, als die Funktion $b_{m, i j}$ im Integral (3.15) entlang der Seiten der Elemente Pole aufweist, wenn der Ansatz (4.1) benutzt wird. Bevor diese Schwierigkeit auf mathematischem weg behoben wird, sollen die 
uleichgewichtsbedingungen auf anschauliche, "baustatische" Weise hergeleitet werden.

Für jeden Knoten lässt sich mittels einer vertikalen virtuellen Verschiebung eine Gleichgewichtsgleichung formulieren. Aus den Gleichgewichtsgleichungen für die freien Knoten werden die Gleichgewichtsbedingungen (3.17), aus den Gleichgewichtsgleichungen für die Auflagerknoten Reaktionen in Form von vertikalen Einzelkräften resultieren.

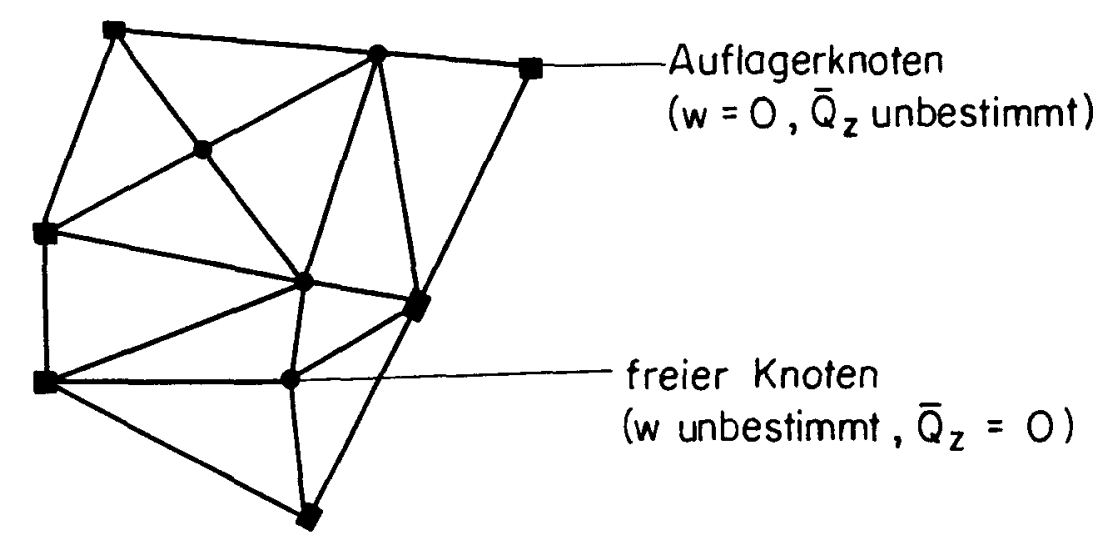

\section{Bild 4.3 : Auflogerknoten und freie Knoten}

Die Gleichgewichtsgleichungen beim Erreichen der Traglast können im folgenden Gleichungssystem zusammengefasst werden:

$$
\left\{\begin{array}{c}
O \\
-\bar{Q}_{z}
\end{array}\right\}=\left[\begin{array}{c}
G_{f} \\
-G_{0}
\end{array}\right]\left\{m_{x-y}\right\}-\lambda \cdot\left\{\begin{array}{c}
P_{f} \\
P_{0}
\end{array}\right\}
$$

Die Dimensionen der Matrizen ergeben sich aus den Dimensionen der Vektoren

$$
\left\{m_{x-y}\right\}_{3 N K}=\left\{\begin{array}{c}
m_{x}^{1} \\
m_{y}^{1} \\
m_{x y}^{1} \\
\hdashline \vdots \\
\vdots \\
\hdashline m_{x}^{N K} \\
m_{y}^{N K} \\
m_{x y}^{N K}
\end{array}\right\}
$$


sowie $\{0\}_{N F K}$ und $\left\{\bar{Q}_{Z}\right\}(N K-N F K)$, wobei mit NK die Anzahl Knoten und mit NFK die Anzahl der freien Knoten bezeichnet wird. Die Reaktionen $\bar{Q}_{z}^{i}$ können erst bestimmt werden, wenn die Momente berechnet sind. Für das lineare Programm ist deshalb nur das Gleichungssystem

$$
\{0\}=\left[G_{f}\right] \quad\left\{m_{x-y}\right\}-\lambda \cdot\left\{p_{f}\right\}
$$

in Betracht zu ziehen.

Die sogenannten globalen Gleichgewichtsmatrizen $\left[G_{f}\right]$ und $\left[G_{a}\right]$ und Lastvektoren $\left\{p_{f}\right\}$ und $\left\{p_{a}\right\}$ ergeben sich wie folgt: Die Koeffizienten $g^{i j}$ resp. $p^{i}$ einer Zeile $i$ des Gleichungssystems sind die Beiträge der Momente resp. der äussern Lasten zur innern resp. äussern Arbeit infolge einer virtuellen Verschiebung $w^{*} i=1$.

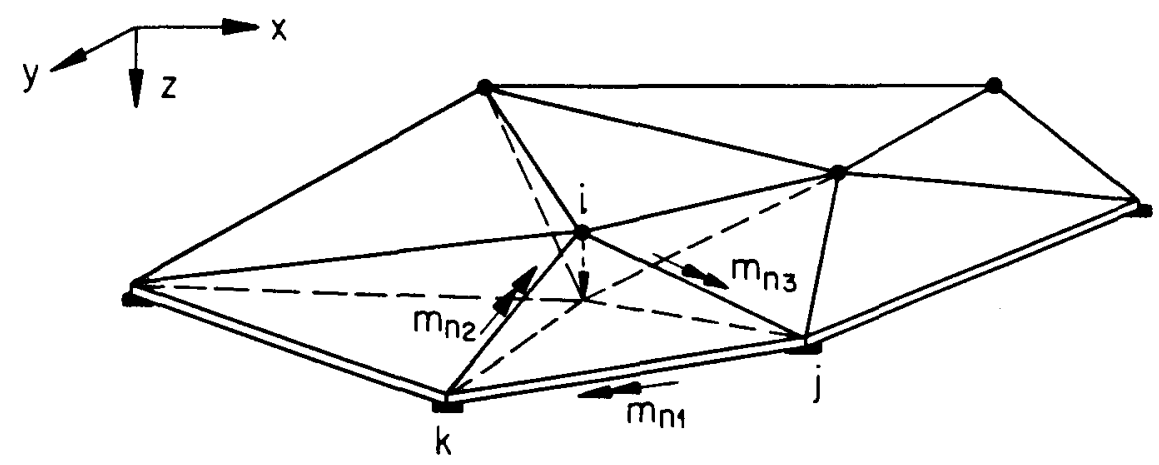

Bild 4.4: Gleichgewicht freier Knoten

Aus dem Bild 4.4 ist leicht ersichtlich, dass diejenigen Koeffizienten $g^{i j}$ einer Zeile $i$ von Null verschieden sind, die in der Kolonne eines Momentes stehen, das einen Beitrag zur innern Arbeit infolge der virtuellen Einheitsverschiebung des Knotens $i$ leistet. Man sieht auch, dass die innere Arbeit am einfachsten in Funktion der Momente $m_{n}$ ausgedrückt wird.

Für die Berechnung mit dem Computer eignet sich am besten ein Verfahren, das demjenigen der "Direct Stiffness Method" gleicht. Zuerst werden die globalen Gleichgewichtsmatrizen und Lastvektoren null gesetzt. Dann werden für jedes Element die zugehörigen Beiträge zur innern und äussern Arbeit infolge der virtuellen Einheitsverschiebungen der Eckpunkte berechnet. Schliesslich werden diese Beiträge am richtigen Ort in die globalen Matrizen addiert. Das Tableau 4.1 zeigt, wo die Beiträge eines Elementes ijk dazugezählt werden. 

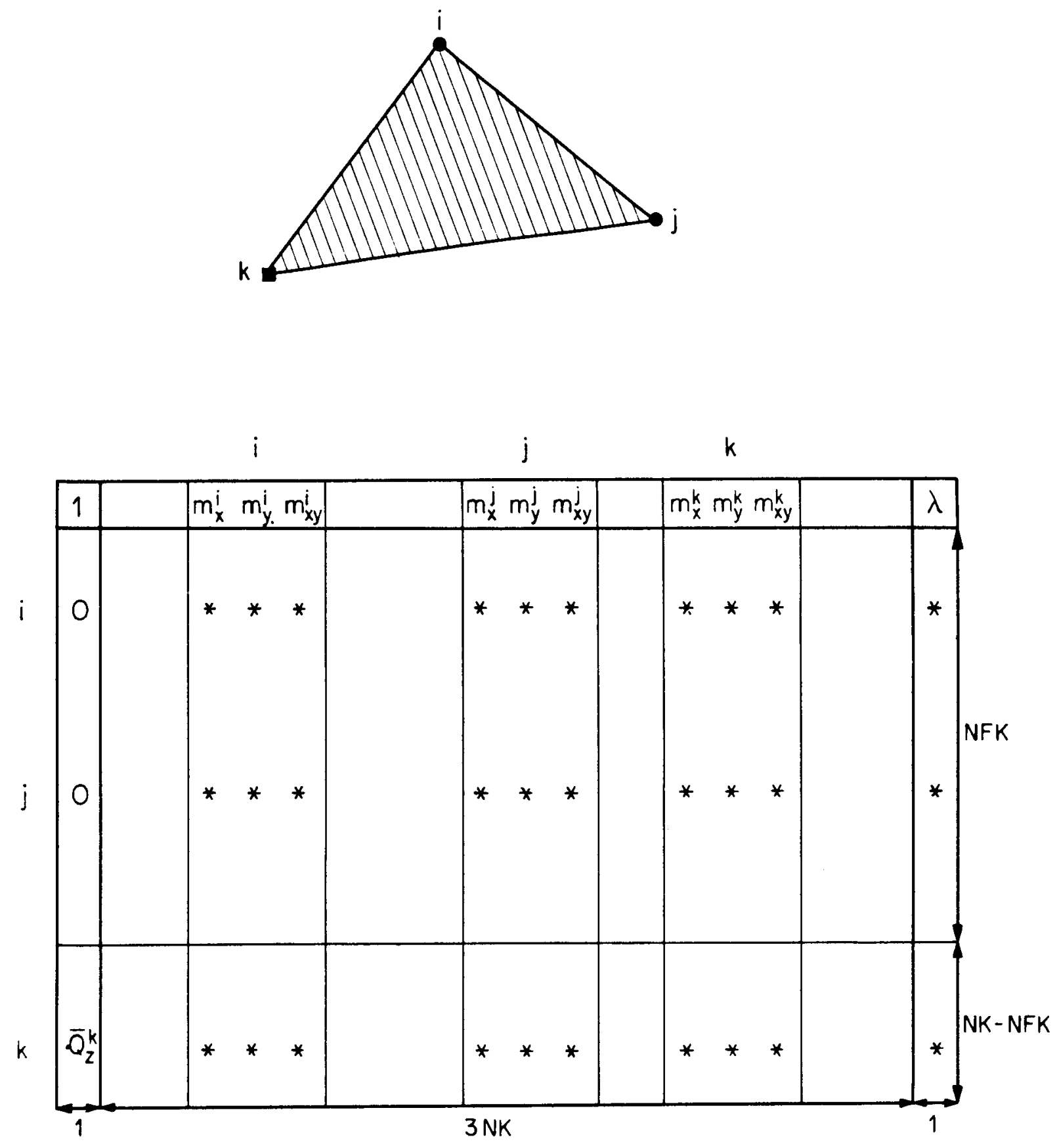

Tableou 4.1: Beiträge eines Elementes.

Das Nullsetzen der Matrizen $\left[G_{f}\right],\left[G_{a}\right],\left\{p_{f}\right\}$ und $\left\{p_{a}\right\}$ bedarf keiner Erläuterung, weil die Dimensionen der Matrizen bereits angegeben wurden. Für die Bestimmung der Beiträge der innern und äussern Arbeit für ein beliebiges Element wird den Knoten und Seiten die lokale Numerierung gemäss Bild 4.5 zugeordnet. 


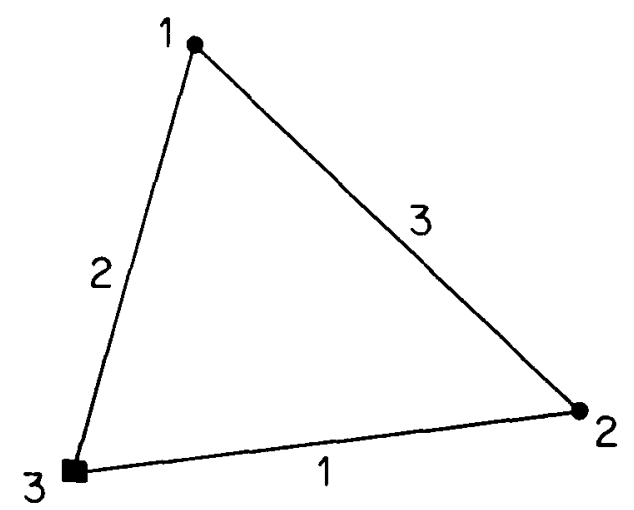

\section{Bild 4.5: Lokale Numerierung}

Die virtuellen Krümmungen treten beim Modell konzentriert entlang der Seiten auf. Der Beitrag eines Elementes zur innern Arbeit $A_{i}^{*}$ infolge einer virtuellen Verschiebung $w^{*} 1$ kann deshalb geschrieben werden als

$$
A_{i}^{* e}\left(w^{* 1}\right)=\oint_{c^{e}} w_{n}^{*}\left(w^{* 1}\right) \cdot m_{n} d s^{e}
$$

Die $\omega_{n}^{*}$ sind die Winkel $w^{*}, n$ entlang der seiten bezüglich der ursprünglichen horizontalen Lage (Bild 4.6) und $C^{e}$ ist der Rand des Elementes. Der Ausdruck (4.6) wird noch mathematisch hergeleitet werden.

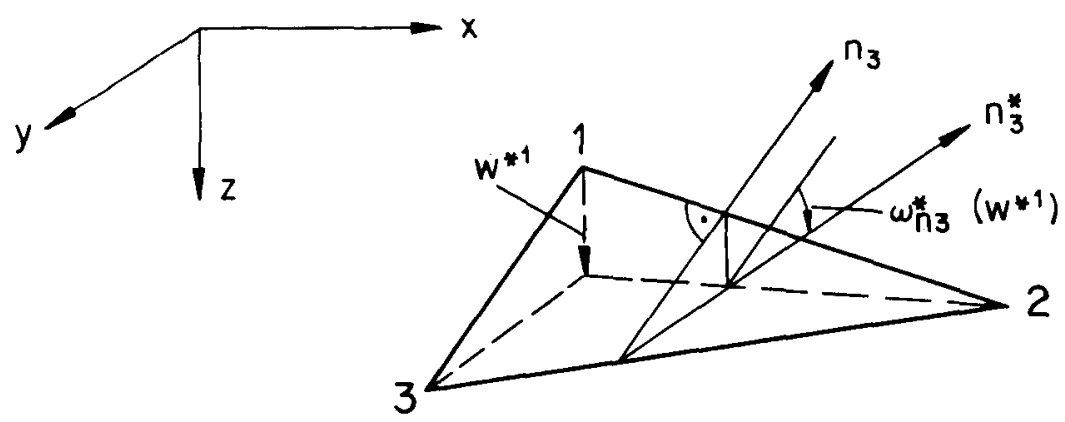

Bild 4.6: Definition von $\omega_{n}^{*}$

Die $\omega_{n}^{*}$ sind pro Seite konstant und lassen sich nach Bild 4.7 geometrisch bestimmen. 


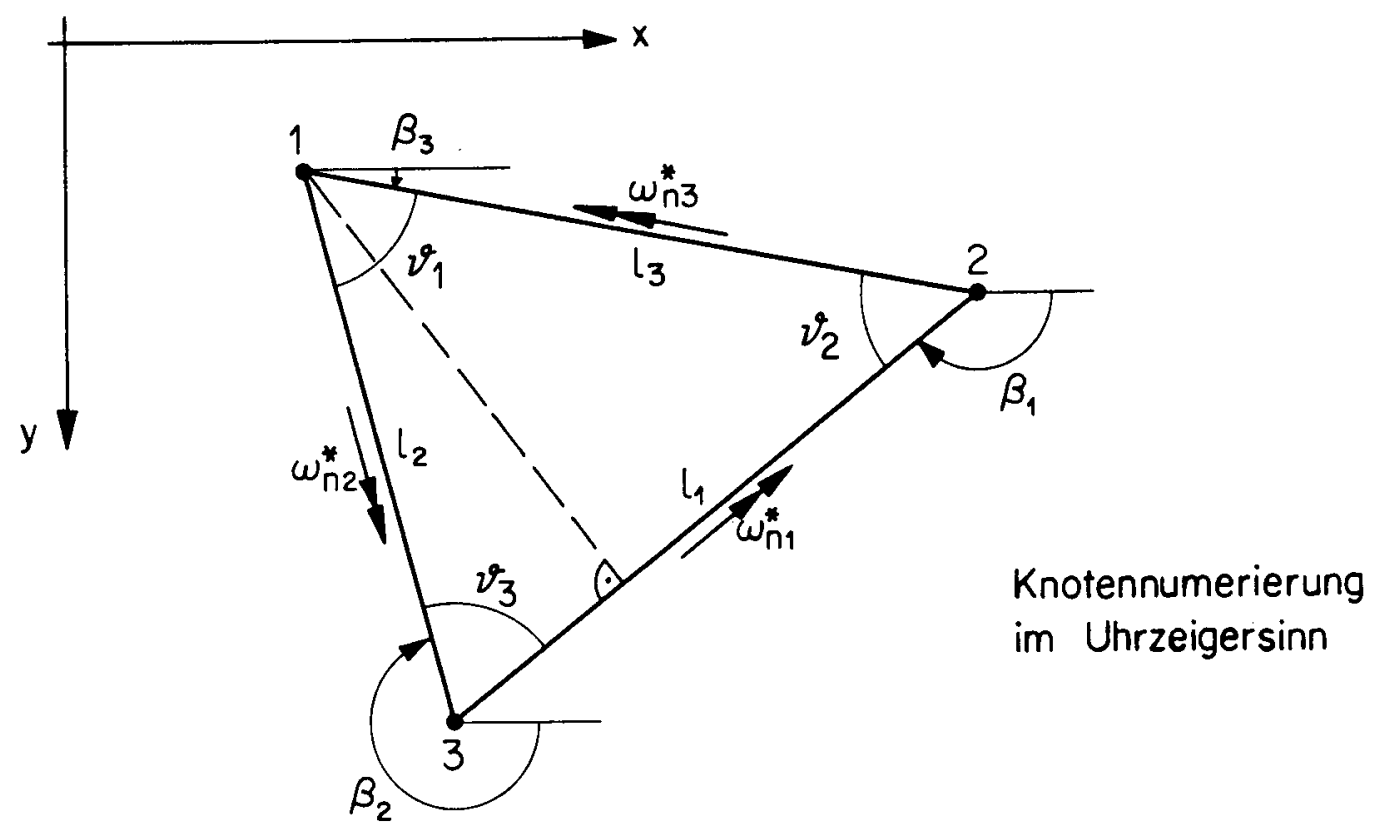

$$
\begin{aligned}
& w^{* 1}=1 \text { (nach unten): } \\
& w_{n 1}^{*}=\frac{-1}{l_{3} \cdot \sin v_{2}}=\frac{-1}{l_{2} \sin v_{3}}, l_{1}=l_{3} \cos v_{2}+l_{2} \cos v_{3} \\
& w_{n 2}^{*}=\frac{\cos v_{3}}{l_{2} \cdot \sin v_{3}}, \quad \omega_{n 3}^{*}=\frac{\cos v_{2}}{l_{3} \sin v_{2}}
\end{aligned}
$$

Bild 4.7: Rotationskomponenten $\omega_{n}^{*}$

Die $\omega_{n d}^{*}(d=1,2,3)$ infolge $w^{*}=1$ sind auch gleich den Reaktionen im Knoten 1 infolge $m_{n d}=1$ an der Seite d für ein Dreieck, das in den knoten vertikal aufgelagert ist (statisch bestimmte Lagerung).

Mit den Abkürzungen

$$
c_{d}=\cot v_{d}, \quad \bar{\omega}_{n d}^{*}=\omega_{n d}^{*} l_{d} \quad(d=1,2,3)
$$

können die Relationen zwischen den Knotenverschiebungen $\left\{w^{*}\right\}$ und den $\left\{\bar{\omega}_{n}^{*}\right\}$ wie folgt geschrieben werden:

$$
\begin{aligned}
& \left\{\begin{array}{l}
\bar{\omega}_{n 1}^{*} \\
\bar{\omega}_{n 2}^{*} \\
\bar{\omega}_{n 3}^{*}
\end{array}\right\}=\left[\begin{array}{ccc}
-\left(c_{2}+c_{3}\right) & c_{3} & c_{2} \\
c_{3} & -\left(c_{3}+c_{1}\right) & c_{1} \\
c_{2} & c_{1} & -\left(c_{1}+c_{2}\right)
\end{array}\right]\left\{\begin{array}{l}
w^{* 1} \\
w^{* 2} \\
w^{* 3}
\end{array}\right\} \\
& \text { oder: }\left\{\bar{\omega}_{n}^{* e}\right\}=\left[Q^{e}\right]\left\{w^{* e}\right\}
\end{aligned}
$$


Die Momente verlaufen entlang der Seiten linear. Die Werte von $m_{n}$ in der Mitte der Seiten ergeben sich aus den Momenten in den Knoten unter Benutzung der Transformation (4.3) zu

$\left\{\begin{array}{l}m_{n 1} \\ m_{n 2} \\ m_{n 3}\end{array}\right\}=\frac{1}{2}\left[\begin{array}{ccccccc}0 & 0 & 0 & \sin ^{2} \beta_{1} & \cos ^{2} \beta_{1}-\sin 2 \beta_{1} & \sin ^{2} \beta_{1} & \cos ^{2} \beta_{1}-\sin 2 \beta_{1} \\ \sin ^{2} \beta_{2} & \cos ^{2} \beta_{2}-\sin 2 \beta_{2} & 0 & 0 & 0 & \sin ^{2} \beta_{2} & \cos ^{2} \beta_{2}-\sin 2 \beta_{2} \\ \sin ^{2} \beta_{3} & \cos ^{2} \beta_{3}-\sin 2 \beta_{3} & \sin ^{2} \beta_{3} & \cos ^{2} \beta_{3}-\sin 2 \beta_{3} & 0 & 0 & 0\end{array}\right]\left\{\begin{array}{l}m_{x}^{1} \\ m_{y}^{1} \\ m_{x y}^{1} \\ m_{x}^{2} \\ m_{y}^{2} \\ m_{x y}^{2} \\ m_{x}^{3} \\ m_{y}^{3} \\ m_{x y}^{3}\end{array}\right\}$

oder: $\left\{m_{n}^{e}\right\}=\left[T_{x-y}^{e}\right]\left\{m_{x-y}^{e}\right\}$

Der Beitrag des Elementes zur äussern Arbeit der Belastung $\lambda_{p}(x, y)$ infolge einer virtuellen Verschiebung $w^{* 1}$ ist

$$
A_{a}^{* e}\left(w^{* 1}\right)=-\iint_{F^{e}} w^{*}\left(w^{* 1}\right) \cdot \lambda p(x, y) d F^{e}
$$

$F^{e}$ ist die Fläche des Elementes. Bei gleichmässig verteilter Belastung $\lambda p_{0}^{e}$ ist z.B.

$$
A_{0}^{* e}\left(w^{* 1}\right)=-\frac{1}{3} \lambda p_{0}^{e} F^{e} w^{* 1}
$$

Die Beiträge eines Elementes zur innern resp. äussern Arbeit infolge von virtuellen Einheitsverschiebungen der Knoten können zu einer "lokalen Gleichgewichtsmatrix" $\left[G^{e}\right]$ resp. zu einem "lokalen Lastvektor" $\left\{p^{\theta}\right\}$ zusammengefasst werden.

$$
\begin{aligned}
& A_{i}^{* e}=\left\{w^{* e}\right\}^{\top} \quad\left[G^{e}\right]_{3 \times 9}\left\{m_{x-y}^{e}\right\} \\
& A_{0}^{* e}=-\left\{w^{* e}\right\}^{\top} \quad\left\{P^{e}\right\}_{3 \times 1}
\end{aligned}
$$


Aus den Gleichungen (4.6) bis (4.8) geht hervor, dass

$$
\left[G^{e}\right]=\left[Q^{e}\right]\left[\begin{array}{l}
T_{x-y}^{e} \\
{[}
\end{array}\right]
$$

Aus der Gleichung (4.10) geht hervor, dass für gleichmässig verteilte Belastung

$$
\left\{p^{e}\right\}=\frac{1}{3} p_{0}^{e} F^{e} \cdot\left\{\begin{array}{l}
1 \\
1 \\
1
\end{array}\right\}
$$

Die Zuordnung der Elementgrössen zu den globalen Grössen (Tableau 4.1) kann - ähnlich wie bei der "Direct Stiffness Method" - mittels Boole'schen Matrizen dargestelit werden.

$$
\begin{aligned}
& \left\{w^{* e}\right\}=\left[L_{w}^{e}\right]_{3 \times N K}\left\{w^{*}\right\} \\
& \left\{m_{x-y}^{e}\right\}=\left[L_{m}^{e}\right]_{9 \times 3 N K}\left\{m_{x-y}\right\}
\end{aligned}
$$

Die Matrizen $\left[L_{W}^{e}\right]$ und $\left[L_{m}^{e}\right]$ sind im Bild 4.8 angegeben. Sie entsprechen den Matrizen $a_{\mathrm{km}}^{e}$ und $\mathrm{b}_{\mathrm{lr}}^{e}$ der allgemeinen topologischen Formulierung im Abschnitt 3.1 (Gleichungen (3.5) bis (3.8)).

Die globale Gleichgewichtsmatrix ergibt sich damit aus

$$
\begin{aligned}
A_{i}^{*}=\sum_{e} A_{i}^{*} & =\sum_{e}\left\{w^{*}\right\}^{\top}\left[L_{w}^{e}\right]^{\top}\left[Q^{e}\right]\left[T_{x-y}^{e}\right]\left[L_{m}^{e}\right]\left\{m_{x-y}\right\} \\
& =\left\{w^{*}\right\}^{\top}\left[\begin{array}{c}
G_{f} \\
\hdashline G_{0}
\end{array}\right]\left\{m_{x-y}\right\}
\end{aligned}
$$

als die folgende Summe:

$$
\left[\begin{array}{c}
G_{f} \\
\hdashline G_{a}
\end{array}\right]=\sum_{e}\left[L_{w}^{e}\right]^{\top}\left[Q^{e}\right]\left[T_{x-y}^{e}\right]\left[L_{m}^{e}\right]
$$




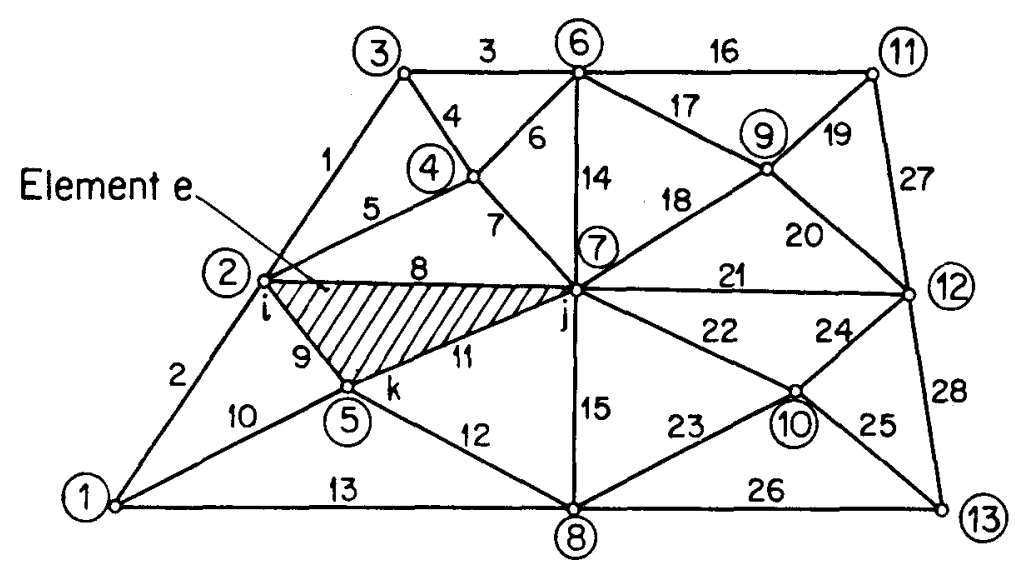

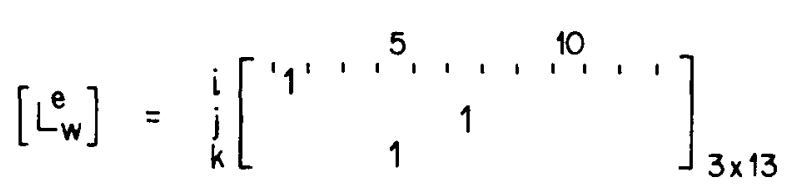

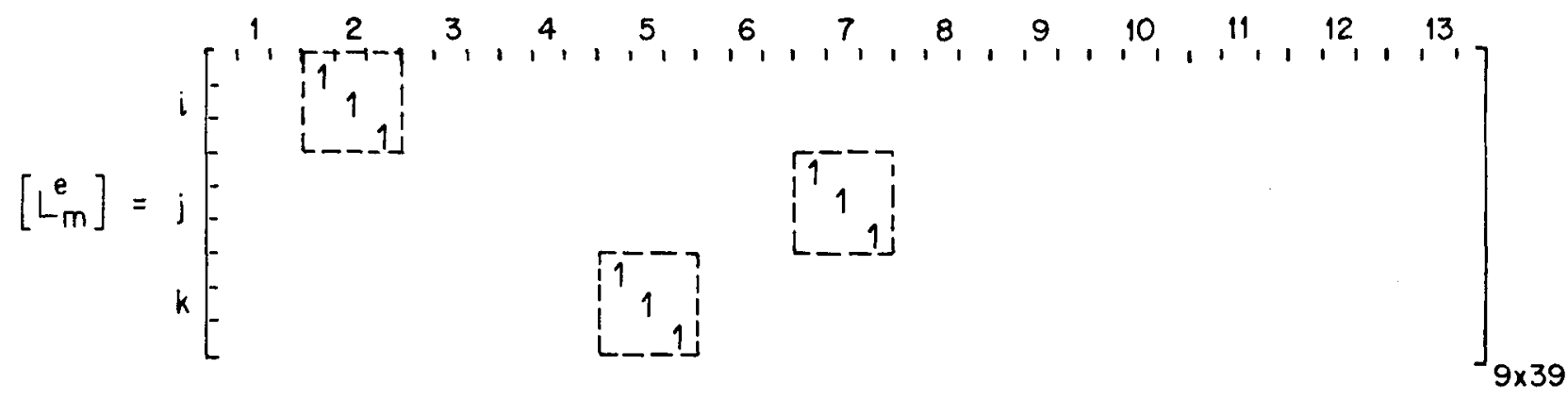

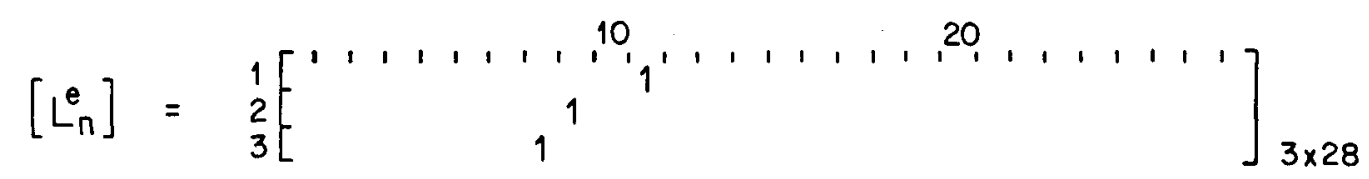

Bild 4.8: Definition der Boole'schen Matrizen

Auf die gleiche Weise erhält man den globalen Lastvektor zu

$$
\left\{-P_{f}\right\}=\sum_{e}\left[L_{w}^{e}\right]^{\top}\left\{p^{e}\right\}
$$

Bei der effektiven Berechnung werden diese Matrix-Multiplikationen selbstverständlich nicht ausgeführt. Die Beiträge der Elemente werden einfach am richtigen Ort in die globalen Matrizen addiert.

Aus der Gleichung (4.4) können ferner vertikale Kräfte $\bar{Q}_{z}$ in den Auflagerknoten als resultierende Reaktionen berechnet werden. 


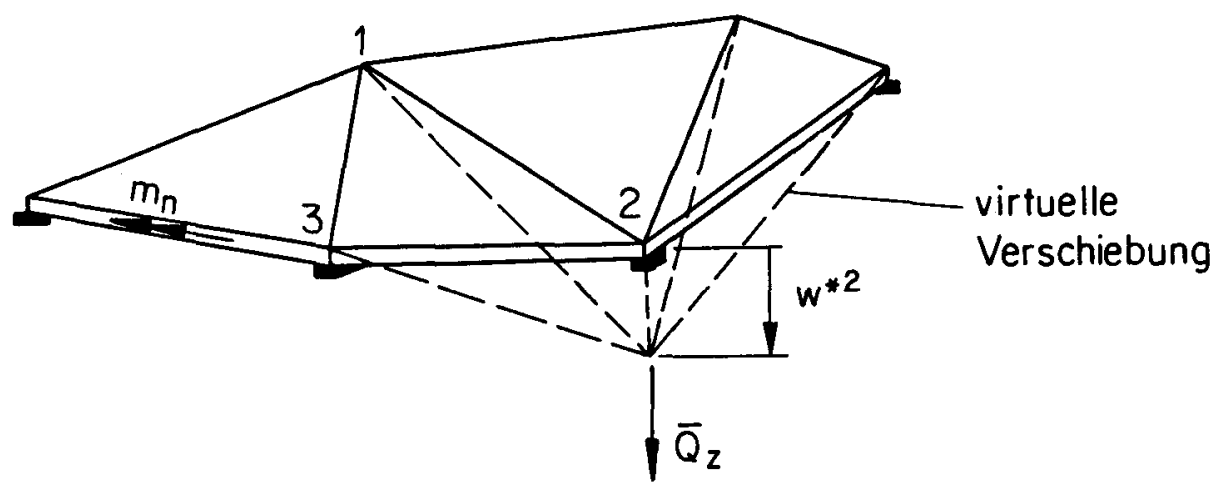

Bild 4.9: Reoktionen

\subsection{Mathematische Formulierung des Gleichgewichtes}

Das Gleichgewicht wird mittels stückweise linearer virtueller Verschiebungen $w^{*}(x, y)$ kontrolliert:

$$
-\iint_{F}\left(m_{x} w_{, x x}^{*}+2 m_{x y} w_{, x y}^{*}+m_{y} w_{i y y}^{*}\right) d x d y-\lambda \iint_{F} p(x, y) w^{*} d x d y=0
$$

Mit $F$ wird die Mittelebene der Platte bezeichnet. Die Gleichung (4.18) könnte auch als Variationsgleichung formuliert werden [23, S. 155]. Sie kann nicht direkt ausgewertet werden, weil die virtuellen Krümmungen ${ }^{*}{ }^{*}, n n$ entlang der Seiten der Elemente unendlich gross sind. Im Anhang B wird gezeigt, dass man durch partielle Integrationen eine Bestätigung der Gleichung (4.6) erhält.

$$
\sum_{e} \oint_{c^{e}} m_{n}^{e} w_{n}^{* e} d s^{e}-\lambda \iint_{F} p w^{*} d F=0
$$

Damit sind die Gleichgewichtsbedingungen des Modelles mathematisch hergeleitet.

Weiter ist zu untersuchen, ob die NK-NFK Gleichgewichtsbedingungen des Modelles eine exakte Erfüllung der allgemein gültigen Gleichgewichtsbedingungen (4.18) gewährleisten. Dazu betrachtet man diejenigen Lasten $\lambda p_{e}(x, y)$, die mit dem Momentenfeld des Modelles im Gleichgewicht stehen (Gleichgewichtslasten), und überprüft, ob sie den äussern Lasten $\lambda p(x, y)$ und den 
Reaktionen entsprechen. Wenn $\lambda p(x, y)=\lambda p_{e}(x, y)$ ist, dann ist das Momentenfeld mit den äussern Lasten im Gleichgewicht.

Die Gleichgewichtslasten lassen sich aus der Anschauung gewinnen oder mathematisch herleiten (Anhang B, Gleichung (B.18)). Sie sind aus dem Bild 4.10 ersichtlich: gleichmässig verteilte Lasten infolge $m_{n, n}$ entlang allen Seiten, gleichmässig verteilte Lasten entlang des Randes infolge $m_{n s, s}$ und Einzellasten in den Randknoten, wenn $m_{n s}$ dort diskontinuierlich ist (Ecke).

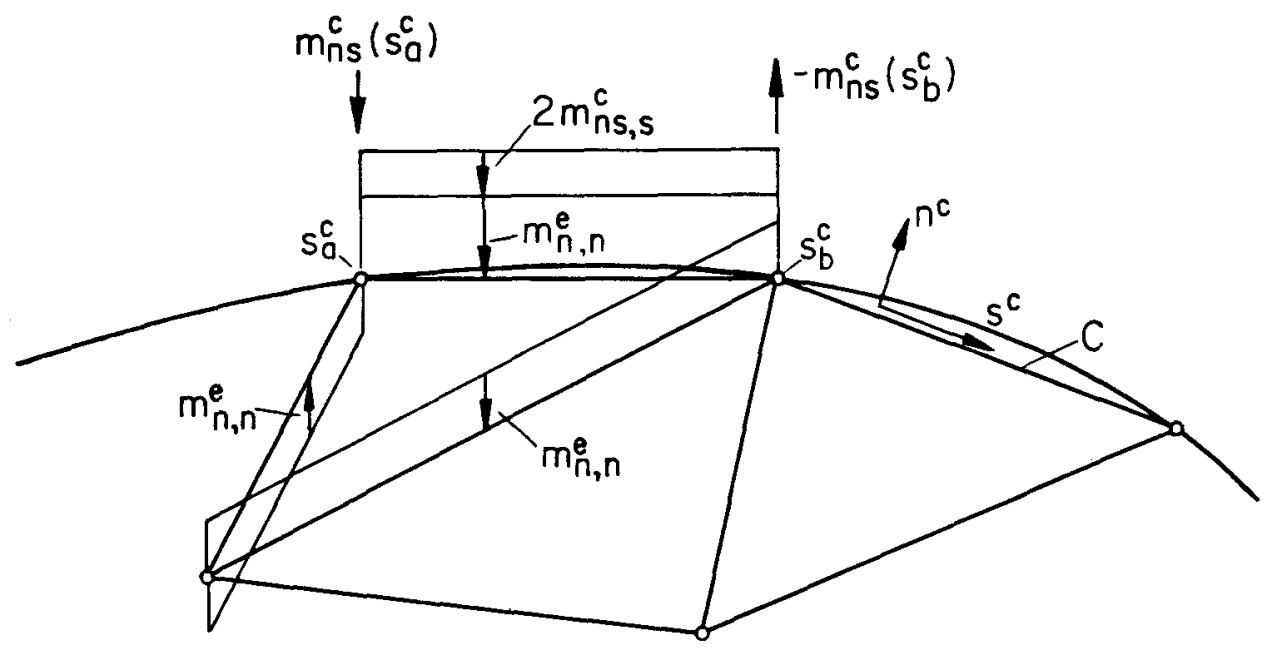

Bild 4.10: Gleichgewichtslosten für ein Element

Man sieht, dass die Gleichgewichtslasten den äussern Lasten im allgemeinen nicht entsprechen. Die Gleichung (4.19) gewährleistet nur, dass die Gleichgewichtslasten bei stückweise linearen virtuellen Verschiebungen die gleiche Arbeit leisten wie die äussern Lasten. Bei einem freien Knoten einer Platte, die nur mit Einzellasten $\lambda P$ in den Knoten belastet ist (Bild 4.11) bedeutet das beispielsweise, dass Betrag und Richtung von $\lambda P$ und der Resultierenden $\lambda P_{e}$ der Linienlasten $\frac{1}{2} \lambda p_{e}(s)$ übereinstimmen, dass aber $\lambda P_{e}$ im allgemeinen neben dem knoten angreift. 


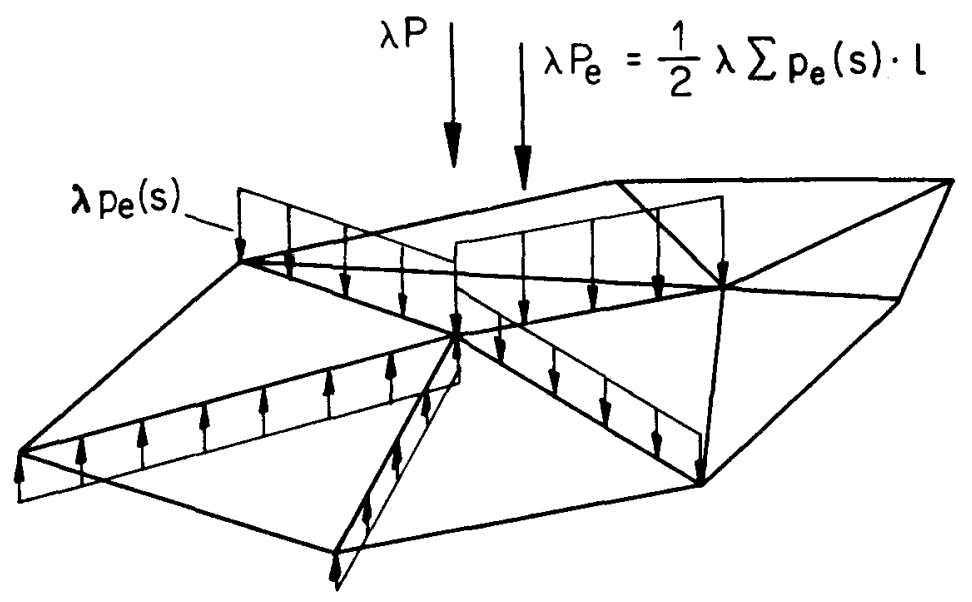

Bild 4.11: Resultierende der Gleichgewichtslosten

Eine vollständige Kontrolle des Gleichgewichtes ist nicht möglich. Es wären NS $\cong 3$ NK Gleichgewichtsbedingungen nätig, um die NS Gleichgewichtslasten zu kontrollieren. Damit wäre das Problem für eine Einteilung in sehr viele Elemente statisch bestimmt, d.h. alle Momentenparameter könnten aus Gleichgewichtsgleichungen allein bestimmt werden.

Das Gleichgewicht wird also beim Modell "TLL" mit stückweise linearem Verlauf der Momente durch äussere Lasten, die nicht mit den Gleichgewichtslasten übereinstimmen können, lokal verletzt. Es kann weder ein aberer noch ein unterer Grenzwert berechnet werden, sondern nur eine Approximation der Traglast. Die resultierende Traglast ist aber ein unterer Grenzwert für die Gleichgewichtslasten und die Resultate werden befriedigend genau, weil die Verletzungen des Gleichgewichtes nur lokal sind.

\section{4 Randbedingungen}

Die geometrischen Randbedingungen $w^{*} k=0$ bewirken, wie im Abschnitt 4.2 festgestellt wurde, dass für die Auflagerknoten keine Gleichgewichtsrestriktion auftritt.

Die statischen Randbedingungen werden gemäss der Gleichung (B.8) im Anhang B berücksichtigt, indem man die entsprechenden Biegemomente null setzt. Die zugehörigen Kolonnen im Gleichungssystem und bei den Plastizitätsbedingungen (Tableau 3.1) können also weggelassen werden. Für die Berechnung mit dem Computer könnte man die verschwindenden Momentenparameter mit einem Index versehen, der anzeigt, dass sie für die weitere Berechnung ohne Belang sind. 
Die Einsparung an Speicherplatz und Rechenzeit durch die Neuordnung der Parameter ist von viel grösserer Bedeutung als bei der Berechnung des elastischen Verhaltens, weil hier nicht mit Bandmatrizen gearbeitet werden kann. Ein Beispiel dafür wird im Abschnitt 8.5 gezeigt.

Die neu geordnete Beziehung (4.5) lautet

$$
\{0\}=\left[\begin{array}{l:l}
G_{0} & G_{x-y}
\end{array}\right]\left\{\begin{array}{c}
0 \\
\hdashline \bar{m}_{x-y}
\end{array}\right\}-\lambda\{p\}
$$

Das Gleichungssystem reduziert sich damit auf

$$
\{0\}=\left[G_{x-y}\right]\left\{\bar{m}_{x-y}\right\}-\lambda\{p\}
$$

Die möglichen Randbedingungen sind im Bild 4.12 zusammengestelit.

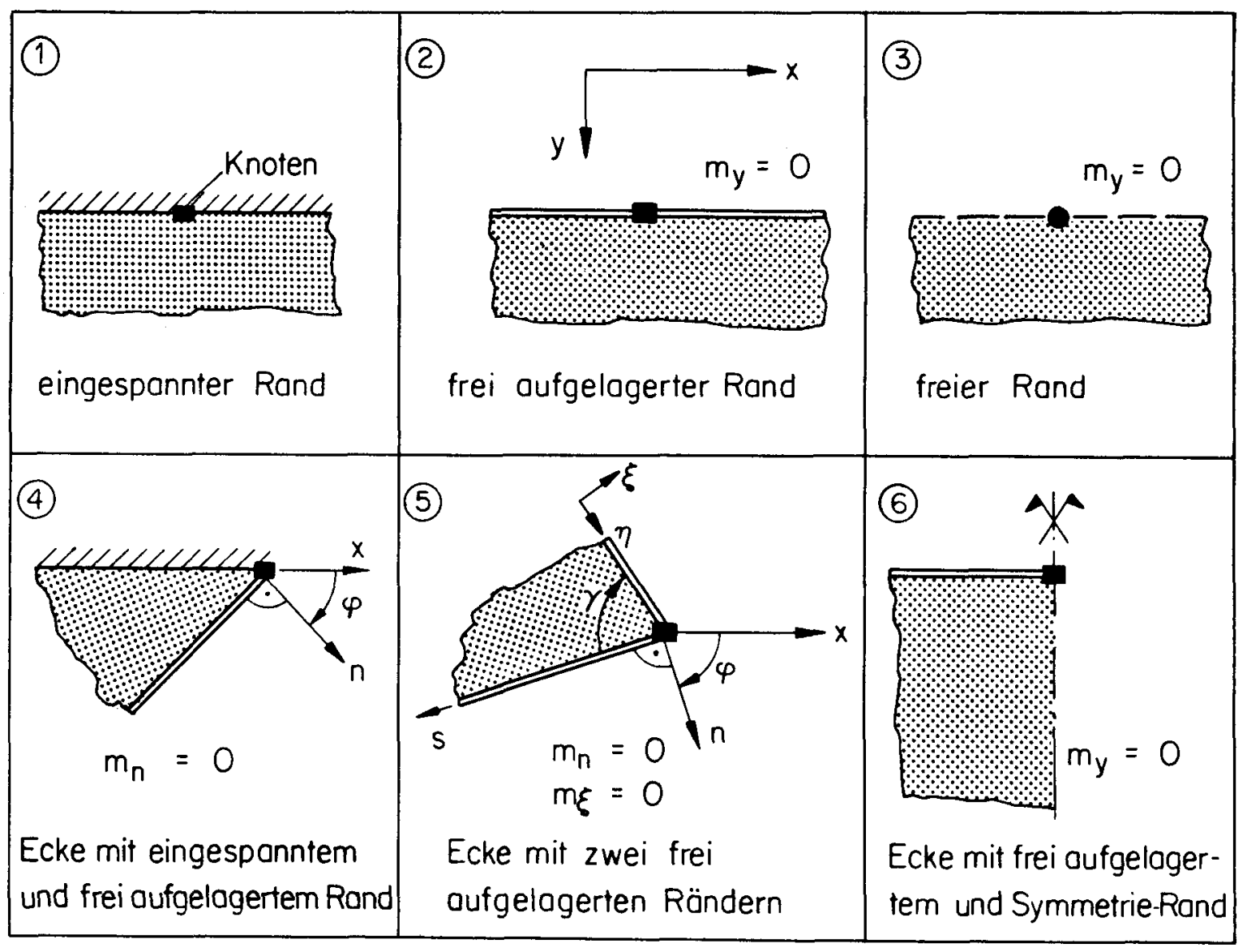

Bild 4.12: Randbedingungen 
In den ersten drei Beispielen ist der Rand parallel zur Koordinatenrichtung

$x$. Ist das nicht der Fall (Beispiel 4), so werden die Momente in diesem Knoten in das (n-s)-Koordinatensystem transformiert (Gleichung (4.2)).

$$
\left\{m_{x-y}^{P}\right\}=\left[T_{\varphi}\right]^{-1}\left\{m_{n-s}^{P}\right\}
$$

Dabei ist

$$
\left[T_{\varphi}\right]^{-1}=\left[\begin{array}{ccc}
\cos ^{2} \varphi & \sin ^{2} \varphi & -2 \sin \varphi \cos \varphi \\
\sin ^{2} \varphi & \cos ^{2} \varphi & 2 \sin \varphi \cos \varphi \\
\sin \varphi \cos \varphi & -\sin \varphi \cos \varphi & \cos ^{2} \varphi-\sin ^{2} \varphi
\end{array}\right]
$$

Es können Randbedingungen für eine beliebige Richtung $n$ und auch Bedingungen für Momente im Innern der Platte formuliert werden.

Wenn auch für den zweiten Rand einer schiefwinkligen Plattenecke eine Randbedingung zu erfüllen ist (Beispiel 5), wird die sich daraus ergebende Beziehung direkt in die Gleichgewichtsmatrix eingesetzt. Es sei $m_{n}=m_{\xi}=0$. Aus

$$
m_{\xi}=m_{n} \cos ^{2} \gamma+m_{s} \sin ^{2} \gamma+2 m_{n s} \sin \gamma \cos \gamma=0
$$

bekommt man

$$
m_{\mathrm{s}}=-m_{\mathrm{ns}} \frac{2 \sin \gamma \cos \gamma}{\sin ^{2} \gamma}=-m_{\mathrm{ns}} \frac{2}{\tan \gamma}
$$

Die Koeffizienten der Gleichgewichtsmatrix in der Kolonne $j$ von $m_{s}$ können also mit denjenigen in der kolonne $k$ von $m_{n s}$ verrechnet werden, indem die $g_{i k}(i=1$, NFK) ersetzt werden durch

$$
g_{i k}+g_{i j} \cdot \frac{-2}{\tan \gamma}
$$

Eigentlich wären jetzt die entsprechenden Kolonnen der Ungleichungsmatrix (Plastizitätsbedingung) zu modifizieren. In diesen Plastizitätsbedingungen 
kommt jedoch nur noch ein Moment vor. Es ist daher von Vorteil, die plastischen Widerstände zu modifizieren, so dass die einfache Form der Ungleichungsmatrix erhalten bleibt.

$\begin{array}{ll}\text { I } & P_{n}+P_{n s}-0-m_{n s} \geqq 0 \\ \text { II } & P_{n}-P_{n s}-0+m_{n s} \geqq 0 \\ \text { III } & P_{s}+P_{n s}-m_{s}-m_{n s} \geqq 0 \\ \text { IV } & P_{s}-P_{n s}-m_{s}+m_{n s} \geqq 0 \\ \text { Z } & N_{n}+N_{n s}+0-m_{n s} \geqq 0 \\ \text { ZII } & N_{n}-N_{n s}+0+m_{n s} \geqq 0 \\ \text { ZII } & N_{s}+N_{n s}+m_{s}-m_{n s} \geqq 0 \\ \text { ZIII } & N_{s}-N_{n s}+m_{s}+m_{n s} \geqq 0\end{array}$

Mit Gleichung (4.25)

$$
\begin{array}{ll}
\text { III } & P_{s}+P_{n s}-m_{n s}(-2 / \tan \gamma+1) \geqq 0 \\
\text { IV } & P_{s}-P_{n s}+m_{n s}(2 / \tan \gamma+1) \geqq 0 \\
\text { III } & N_{s}+N_{n s}-m_{n s}(2 / \tan \gamma+1) \geqq 0 \\
\text { IIII } & N_{s}-N_{n s}+m_{n s}(-2 / \tan \gamma+1) \geqq 0
\end{array}
$$

Der Ausdruck in der klammer wird mit $k$ bezeichnet; er ist negativ, wenn

$$
\begin{aligned}
0 & <\tan y<2 \\
-2 & <\tan \gamma<0 \\
-2 & <\tan \gamma<0 \\
0 & <\tan \gamma<2
\end{aligned}
$$

Wenn $k>0$ ist, können die Ungleichungen durch $k$ dividiert werden, ohne dass sich das Ungleichungszeichen ändert. Für $k=0$ ist die entsprechende Plastizitätsbedingung für jedes $m_{n s}$ erfüllt; $m_{n s}$ ist aber durch die übrigen Plastizitätsbedingungen beschränkt. 
diert, d.h. das Vorzeichen von $m_{n s}$ ändert sich. Zum Beispiel:

$$
\begin{aligned}
& \text { III } \quad P_{s}+P_{n s}-m_{n s} \cdot k \geqq 0 \\
& \text { VIII } \quad N_{s}-N_{n s}+m_{n s} \cdot k \geqq 0 \\
& \text { III } \quad \frac{1}{-k}\left(P_{s}+P_{n s}\right)+m_{n s} \geqq 0 \rightarrow \text { VIII } \\
& \text { VIII } \frac{1}{-K}\left(N_{s}-N_{n s}\right)-m_{n s} \geqq 0 \rightarrow \text { III }
\end{aligned}
$$

Die einfache Form der Plastizitätsbedingungen für einen Knoten

$$
\left\{\begin{array}{l}
c_{I} \\
\cdot \\
\cdot \\
\cdot \\
\cdot \\
c_{\text {III }}
\end{array}\right\}+\left[\begin{array}{rrr}
-1 & 0 & -1 \\
-1 & 0 & 1 \\
0 & -1 & -1 \\
0 & -1 & 1 \\
1 & 0 & -1 \\
1 & 0 & 1 \\
0 & 1 & -1 \\
0 & 1 & 1
\end{array}\right]\left\{\begin{array}{l}
m_{n} \\
m_{s} \\
m_{n s}
\end{array}\right\} \geqq\left\{\begin{array}{l}
0 \\
\cdot \\
\cdot \\
\cdot \\
\cdot \\
\cdot \\
\cdot \\
0
\end{array}\right\}
$$

bleibt somit erhalten, wenn man zwei modifizierte plastische Widerstände vertauscht, z.B.:

$$
\begin{aligned}
& c_{\text {III }}=\frac{1}{-k}\left(N_{s}-N_{n S}\right) \\
& c_{\text {IIII }}=\frac{1}{-k}\left(P_{s}+P_{n s}\right)
\end{aligned}
$$

Das Gleichungssystem (4.21) wird durch die Variablentransformation (4.22) und die soeben gezeigte Verrechnung der Koeffizienten abhängiger Parameter zu

$$
\{0\}=[G]\{m\}-\lambda\{p\}
$$




\subsection{Plastizitätsbedingungen}

Die Momente verlaufen stückweise linear und sind kontinuierlich. Es genügt deshalb, die linearen Plastizitätsbedingungen (2.15) in den Knoten zu kontrollieren. Die Momente in den Knoten sind nach Gleichung (3.19) allgemein

$$
\left\{m^{q}\right\}=\left[\psi^{q}\right]\{m\}
$$

Da die Variablen bei diesem Modell gerade den Momenten in den Knoten entsprechen, wird $\left[\psi^{Q}\right]$ für den Knoten $q=j$ eine einfache Boole'sche Zuordnungsmatrix

$$
\left[\psi^{q}\right]=\left[L_{m}^{j}\right]
$$

Sie besteht aus den drei Zeilen der Matrix $\left[L_{m}^{e}\right]$ (Bild 4.8), die zum Knoten j gehören.

Wenn man die $Q$ Sätze von Ungleichungen (Tableau 3.1) zu einem System von Ungleichungen zusammenfasst, lässt sich die im linearen Programm (3.21) verwendete Ungleichungsmatrix anschreiben als

$$
[U]=\sum_{j}\left[L_{q}^{j}\right]\left[U^{q}\right]_{8 \times 3}\left[L_{m}^{i}\right]_{3 \times 3 N K}
$$

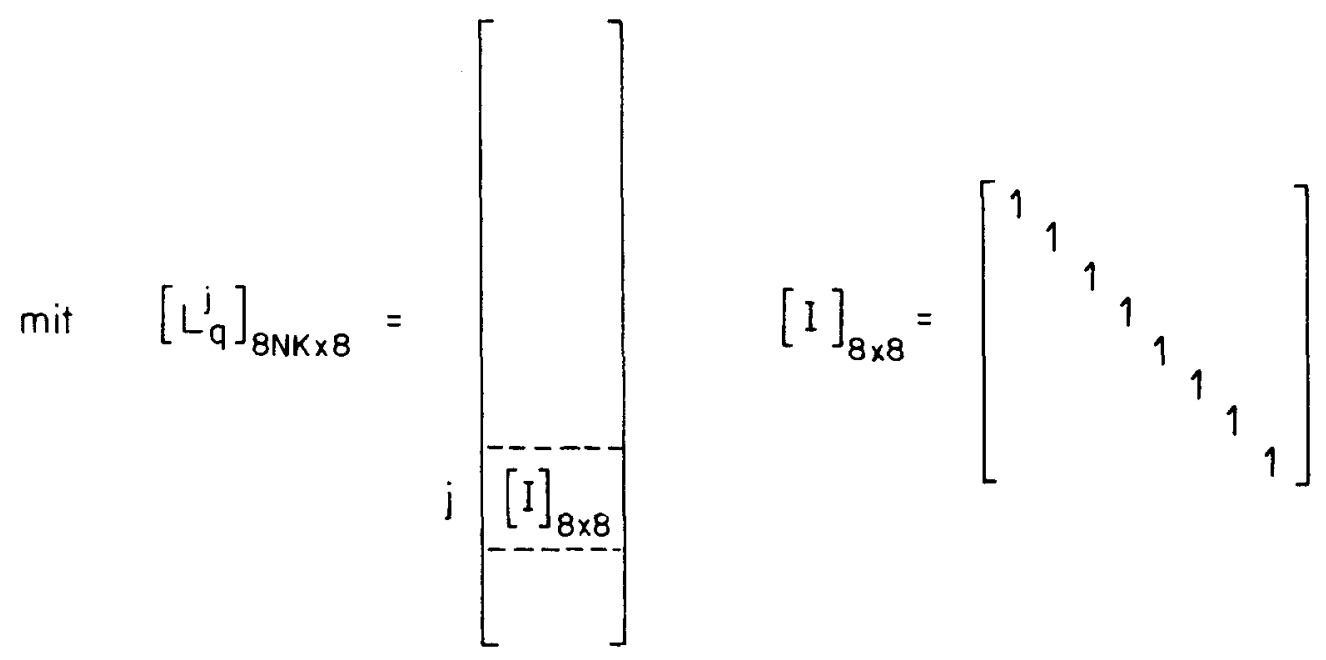

Nicht eingezeichnete Elemente sind null. Der Summationsindex läuft über alle Knoten. 
Die Ungleichungsmatrix ist im Tableau 4.2 für den Fall dargestellt, dass keine Momente infolge Randbedingungen verschwinden.

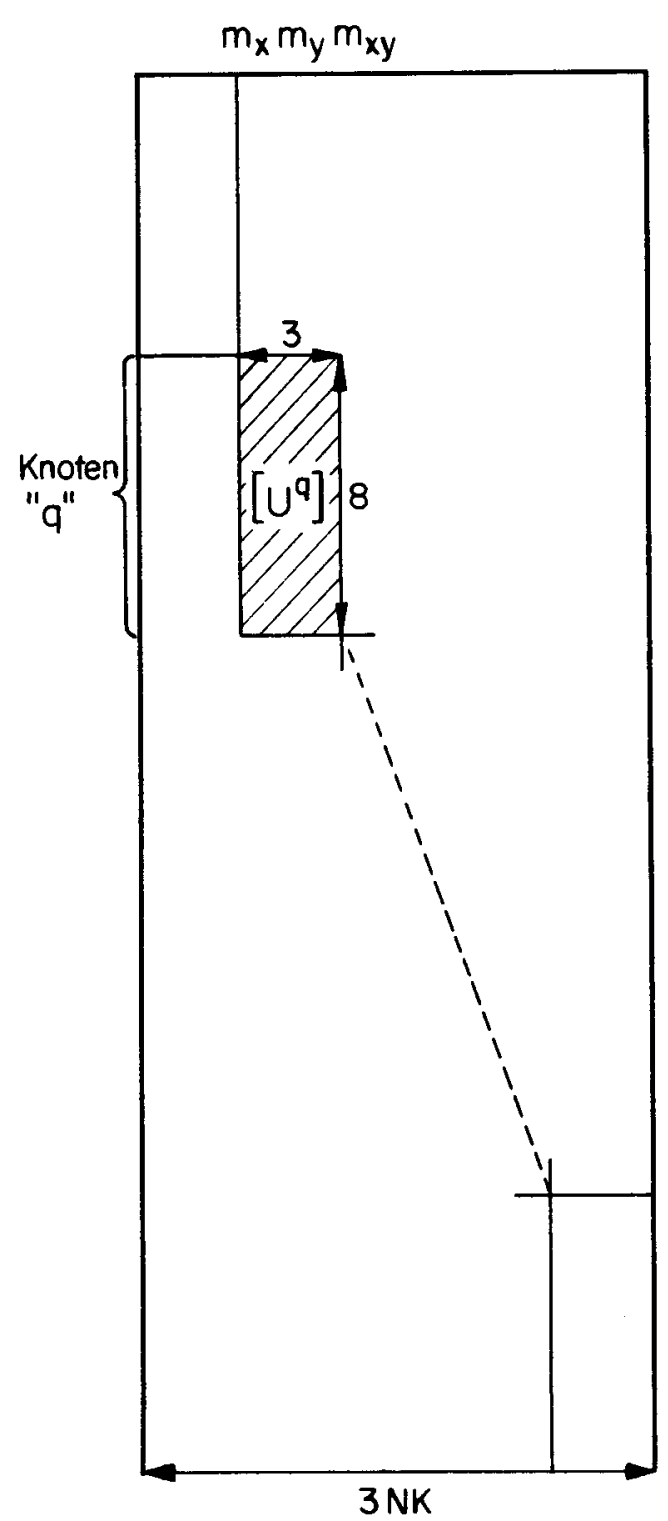

Tableau 4.2: Ungleichungsmatrix

Wenn Randbedingungen auftreten, sind die entsprechenden Kolonnen wegzulassen. Das resultierende System von Ungleichungen wird allgemein geschrieben als

$$
[U]\{m\}+\{c\} \geqq\{0\}
$$


5.1 Ansatz für die Momente

Die Momente $m_{x}, m_{y}$ und $m_{x y}$ werden als konstant innerhalb eines dreieckigen finiten Elementes angenommen. Als zusätzliche Bedingung müssen die im Element ebenfalls konstanten Momente $m_{n}$ für je zwei angrenzende Elemente (+) und (-) gleich sein, weil die Ableitung $w$, n der Verschiebungsfunktion entlang der Seiten diskontinuierlich ist.

Bild 5.1: Sponnungsansatz

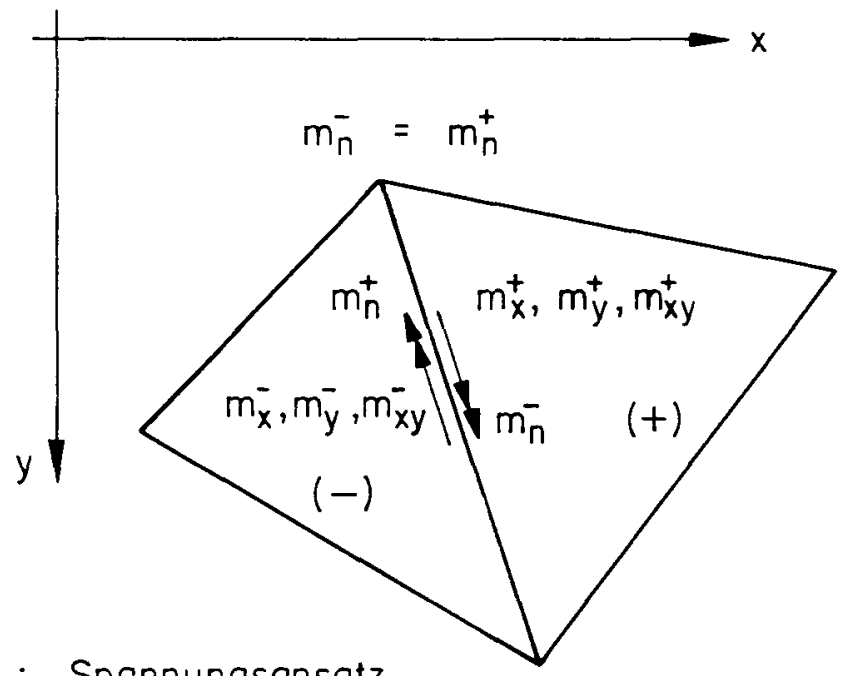

Deshalb werden nicht die Momente $m_{x}^{e}, m_{y}^{e}, m_{x y}^{e}(e=1$, NE) als Parameter gewählt, sondern die Momente $m_{n}^{d}(d=1$, NS). Mit NE wird die Anzahl Elemente, mit NS die Anzahl Seiten und mit NK die Anzahl Knoten bezeichnet. Für eine grosse Anzahl von Elementen gelten die Beziehungen NE $\cong 2$ und NS $\cong 1,5 \mathrm{NE} \cong 3 \mathrm{NK}$.

Im folgenden werden die im Bild 5.2 angegebenen Bezeichnungen verwendet. 


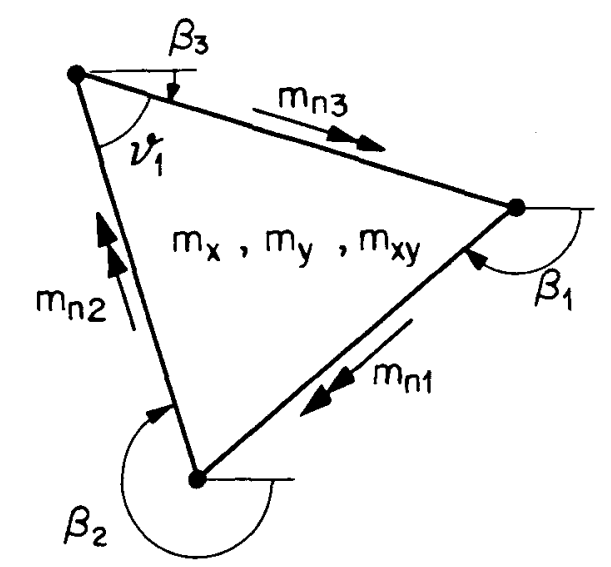

\section{Bild 5.2: Bezeichnungen}

Der Zusammenhang zwischen den Momenten ist gegeben durch die erste Gleichung von (4.3) für das infinitesimale Gleichgewicht.

$$
\begin{aligned}
m_{n 1} & =m_{x} \sin ^{2} \beta_{1}+m_{y} \cos ^{2} \beta_{1}-2 m_{x y} \sin \beta_{1} \cos \beta_{1} \\
m_{n 2} & =m_{x} \sin ^{2} \beta_{2}+m_{y} \cos ^{2} \beta_{2}-2 m_{x y} \sin \beta_{2} \cos \beta_{2} \\
m_{n 3} & =m_{x} \sin ^{2} \beta_{3}+m_{y} \cos ^{2} \beta_{3}-2 m_{x y} \sin \beta_{3} \cos \beta_{3} \\
& \text { oder: }\left\{m_{n}^{e}\right\}=\left[T_{n}\right]_{3 x 3}\left\{m_{x-y}^{e}\right\}
\end{aligned}
$$

Da die Matrix $\left[_{n}\right]$ nichtsingulär ist (umkehrbar eindeutiger Zusammenhang), können aus den Normalmomenten wieder die Momente im Element berechnet werden:

$$
\left\{m_{x-y}^{e}\right\}=\left[T_{n}\right]_{3 \times 3}^{-1}\left\{m_{n}^{e}\right\}
$$

Die Drillungsmomente erhält man aus den Transformationsformeln

$$
\begin{aligned}
m_{n s 1} & =m_{x} \sin \beta_{1} \cos \beta_{1}-m_{y} \sin \beta_{1} \cos \beta_{1}-m_{x y}\left(\cos ^{2} \beta_{1}-\sin ^{2} \beta_{1}\right) \\
m_{n s 2} & =m_{x} \sin \beta_{2} \cos \beta_{2}-m_{y} \sin \beta_{2} \cos \beta_{2}-m_{x y}\left(\cos ^{2} \beta_{2}-\sin ^{2} \beta_{2}\right) \\
m_{n s 3} & =m_{x} \sin \beta_{3} \cos \beta_{3}-m_{y} \sin \beta_{3} \cos \beta_{3}-m_{x y}\left(\cos ^{2} \beta_{3}-\sin ^{2} \beta_{3}\right) \\
& \text { oder: }\left\{m_{n s}^{e}\right\}=\left[T_{n s}\right]_{3 x 3}\left\{m_{x-y}^{e}\right\}
\end{aligned}
$$


Dieser Zusammenhang ist nicht mehr umkehrbar, weil z.B. bei $v_{1}=\frac{\pi}{2}$ die Momente $m_{n s 2}$ und $m_{n s 3}$ gleich sind und damit [ $T_{n s}$ ] singulär wird.

\subsection{Gleichgewichtsbedingungen}

Es sind die Koeffizienten des Gleichungssystems

$$
\left\{\begin{array}{c}
O \\
\hdashline \bar{Q}_{z}
\end{array}\right\}=\left[\begin{array}{c}
G_{f} \\
\hdashline G_{0}
\end{array}\right]\left\{m_{n}\right\}-\lambda\left\{\begin{array}{c}
p_{f} \\
\hdashline P_{o}
\end{array}\right\}
$$

zu bestimmen. Die Dimensionen der Matrizen in der Gleichung (5.4) ergeben sich aus den Dimensionen der Vektoren $\left\{m_{n}\right\}_{N S}$, $\{0\}_{N F K}$ und $\left\{\bar{Q}_{z}\right\}_{N K-N F K}$, wobei mit NS die Anzahl Seiten, mit NK die Anzahl Knoten und mit NFK die Anzahl freier knoten bezeichnet wird.

Für die Berechnung der Beiträge eines Elementes zur innern und äussern Arbeit wird wie im Abschnitt 4.2 das Prinzip der virtuellen Verschiebungen benutzt. Es stellt sich die Frage, ob nicht auch die Drillungsmomente einen Anteil zur innern Arbeit leisten, weil $\mathrm{m}_{\mathrm{ns}}^{-} \neq \mathrm{m}_{\mathrm{ns}}^{+}$ist. Da jedoch $\mathrm{w}^{*}$, ns in der Gleichung (4.18) entlang der Seiten null ist, entsteht keine innere Arbeit. Mit konstanten Drillungsmomenten allein kann kein Gleichgewicht zu vertikalen äussern Lasten innerhalb der Platte hergestellt werden. Bei reiner Torsionsbeanspruchung (Bild 5.3) treten keine äussern Lasten innerhalb der Platte auf.

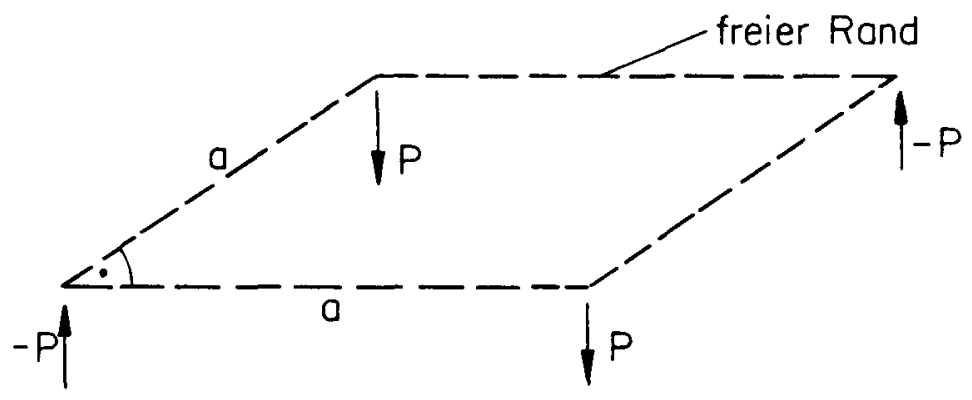

\section{Bild 5.3: Reine Torsionsbeanspruchung}

Der Beitrag eines Elementes zur innern Arbeit infolge einer virtuellen Verschiebung. $w^{* 1}$ ist demnach wieder

$$
A_{i}^{* e}\left(w^{* 1}\right)=\oint_{c^{e}} w_{n}^{*}\left(w^{* 1}\right) \cdot m_{n} d s^{e}
$$


Dieser Ausdruck wird noch mathematisch hergeleitet werden. Die "lokale Gleichgewichtsmatrix" resp. der "lokale Lastvektor" sind definiert durch

$$
\begin{aligned}
& A_{i}^{* e}=\left\{w^{* e}\right\}^{\top}\left[G^{e}\right]_{3 \times 3}\left\{m_{n}^{e}\right\} \\
& A_{0}^{* e}=-\left\{w^{* e}\right\}^{\top}\left\{p^{e}\right\}_{3 \times 1}
\end{aligned}
$$

Die Matrix $\left[G^{e}\right]$ ergibt sich direkt aus der Gleichung (4.7), weil die $m_{n}$ bei diesem Modell als Variable auftreten.

$$
\left[G^{e}\right]=\left[Q^{e}\right]
$$

Im Tableau 5.1 ist angegeben, bei welchen Koeffizienten der globalen Matrizen die Beiträge eines Elementes ijk dazuzuzählen sind. Die Summation über alie Elemente kann auch mittels Boole'scher Matrizen formuliert werden. Die Zuordnung der Elementgrössen zu den globalen Grössen lässt sich darstellen als

$$
\begin{aligned}
& \left\{w^{* e}\right\}=\left[L_{w}^{e}\right]_{3 \times N K}\left\{w^{*}\right\} \\
& \left\{m_{n}^{e}\right\}=\left[L_{n}^{e}\right]_{3 \times N S}\left\{m_{n}\right\}
\end{aligned}
$$

Die Matrizen $\left[\mathrm{L}_{w}^{\mathrm{e}}\right]$ und $\left[\mathrm{L}_{n}^{\mathrm{e}}\right]$ sind im Bild 4.8 angegeben. Sie entsprechen den Matrizen $a_{\mathrm{km}}^{e}$ und $b_{\mathrm{ln}}^{e}$ der allgemeinen topologischen Formulierung im Abschnitt 3.1 (Gleichungen (3.5) bis (3.8)). Die globale Gleichgewichtsmatrix bekommt man somit als

$$
\left[\frac{G_{f}}{G_{a}}\right]=\sum_{e}\left[L_{w}^{e}\right]^{\top}\left[Q^{e}\right]\left[L_{n}^{e}\right]
$$

Die Randbedingungen sind sehr einfach zu berücksichtigen. Man setzt gegebenenfalls $m_{n}=0$. 

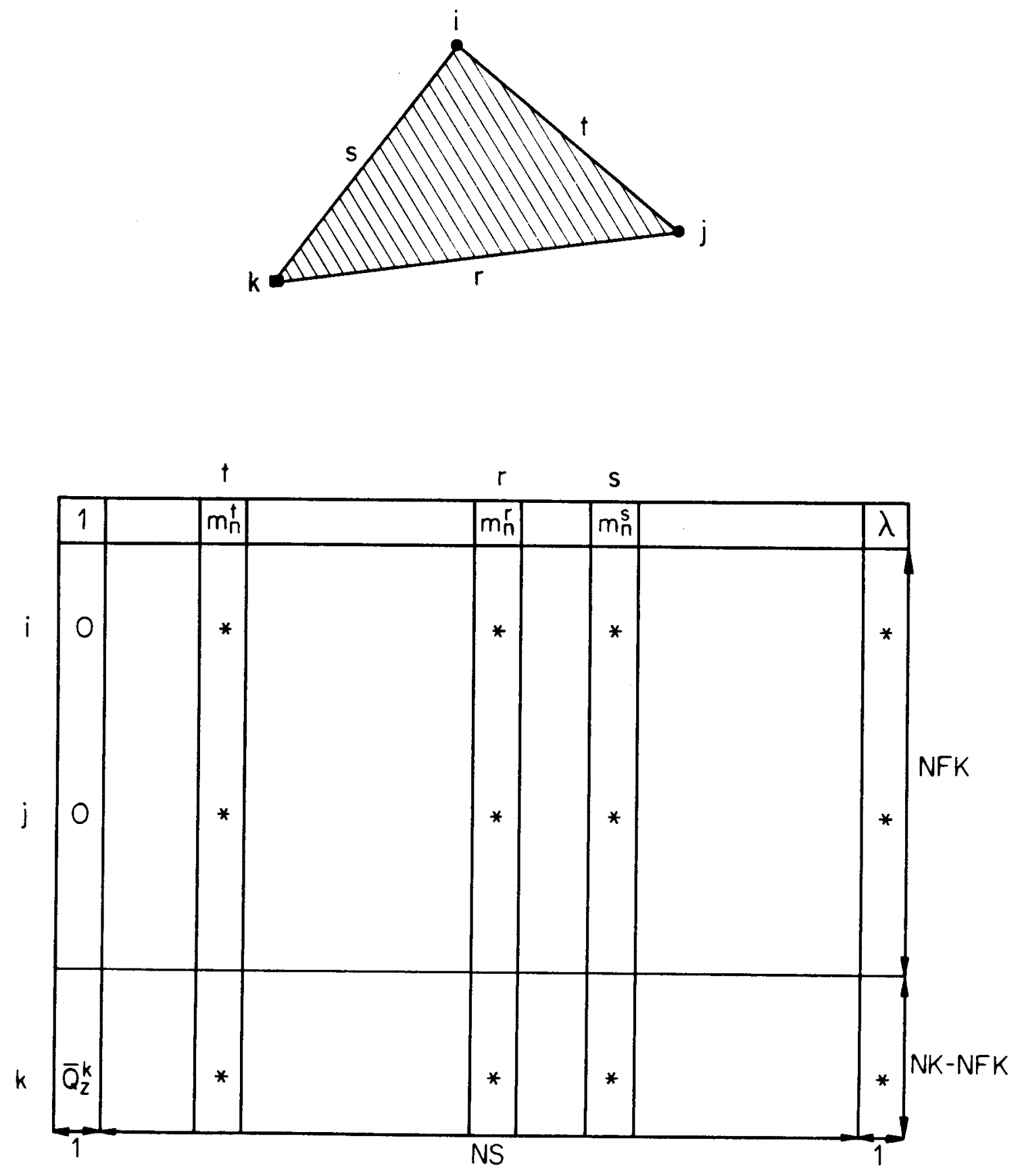

Tableau 5.1: Beiträge eines Elementes 
Die aus der Gleichung (5.4) hervorgehenden Gleichgewichtsbedingungen werden schliesslich in allgemeiner form geschrieben als

$$
\{0\}=[G]\{m\}-\lambda\{p\}
$$

\subsection{Mathematische Formulierung des Gleichgewichtes}

Die Gleichung (4.18) für das Gleichgewicht kann auch für dieses Modell nicht direkt ausgewertet werden, weil die virtuellen krümmungen ${ }^{*}{ }^{*}, n n$ entlang der Seiten der Elemente unendlich gross sind. Im Anhang $B$ wird gezeigt, dass man durch partielle Integration wiederum eine Bestätigung der Gleichung (4.6) erhält

$$
\sum_{e} \oint_{c^{e}} m_{n}^{e} w_{, n}^{* e} d s^{e}-\lambda \iint_{F} p w^{*} d F=0
$$

Damit sind die Gleichgewichtsbedingungen des Modelles mathematisch hergeleitet.

Die Gleichgewichtslasten lassen sich aus der Anschauung gewinnen oder mathematisch herleiten (Anhang B, Gleichung (B.34)). Es sind bei diesem Modell Einzellasten in den Knoten, weil die Drillungsmomente im Rahmen der Kirchhoff'schen Plattentheorie durch statisch gleichwertige Schubkräfte ersetzt werden können.

Wenn die äussere Belastung aus Knotenlasten besteht, ist somit $\lambda P^{k}=\lambda P_{e}^{k}$ und das Gleichgewicht ist auch lokal erfülit. Deshalb liefert das Modell mit elementweise konstanten Momenten für diesen Belastungsfall einen untern Grenzwert der Traglast.

\subsection{Plastizitätsbedingungen}

Die Momente sind innerhalb eines Elementes konstant. Die linearen Plastizitätsbedingungen (2.15) sind deshalb in jedem Element zu kontrollieren. Die Momente in einem Element müssen in Funktion der Variablen, also der Momente $m_{n}$ entleng der Seiten ausgedrückt werden. Aus den Beziehungen (5.2) und (5.7) ergitt sich 


$$
\left\{m_{x-y}^{e}\right\}=\left[T_{n}\right]^{-1}\left[L_{n}^{e}\right]\left\{m_{n}\right\}
$$

Der Vergleich mit der Gleichung (3.19) zeigt, dass bei diesem Modell

$$
\left[\psi^{q}\right]=\left[T_{n}\right]^{-1}\left[L_{n}^{e}\right]
$$

Wenn man die $Q$ Sätze von Ungleichungen (Tableau 3.1) zu einem System von Ungleichungen zusammenfasst, lässt sich die im linearen Programm (3.21) verwendete Ungleichungsmatrix unter Benutzung der Abkürzung

$$
\left[U_{n}^{q}\right]=\left[U^{q}\right]\left[T_{n}\right]^{-1}
$$

anschreiben als

$$
[U]=\sum_{\mathbf{e}}\left[L_{q}^{e}\right] \quad\left[U_{n}^{q}\right]_{8 \times 3}\left[L_{n}^{e}\right]_{3 \times N S}
$$

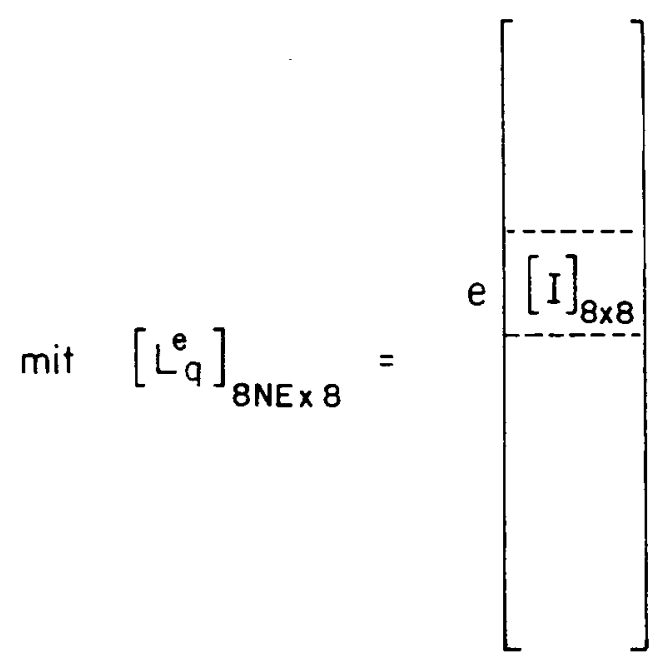

Nicht eingezeichnete Elemente in der Matrix $\left[L_{q}^{B}\right]$ sind null.

Die Koordinatenaxen $x^{e}$ und $y^{e}$ für die lokalen Momente $m_{x-y}^{e}$ (Gleichung 5.9) werden pro Element parallel zu den Hauptrichtungen der Armierung gewählt. Das ist hier im Gegensatz zum Modell "TLL" in jedem Falle möglich, weil für die Randbedingungen keine Rotation des Koordinatensystems nötig ist. Bei verschiedenen Hauptrichtungen von positiver (Index $P$ ) und negativer (Index N) Armierung können die Plastizitätsbedingungen in verschiedener Richtung 
kontrolliert werden:

$$
\begin{aligned}
& \left\{U_{n P}^{q}\right\}=\left[U_{P}^{q}\right]\left[T_{n P}\right]^{-1} \\
& \left\{U_{n N}^{q}\right\}=\left[U_{N}^{q}\right]\left[T_{n N}\right]^{-1} \\
& {\left[U_{n}^{q}\right]=\left[\frac{U_{n P}^{q}}{U_{n N}^{q}}\right]}
\end{aligned}
$$

Die Matrizen $\left[U_{n}^{q}\right]$ müssen während des Optimierungsprozesses (Kapitel 7) verfügbar sein.

Die Ungleichungsmatrix ist im Tableau 5.2 für den Fall dargestellt, dass keine Momente infolge Randbedingungen verschwinden. Wenn Randbedingungen auftreten, ist [U] um die entsprechenden Kolonnen zu reduzieren. Das resultierende System von Ungleichungen kann wieder allgemein bezeichnet werden mit

$$
[U]\{m\}+\{c\} \geqq\{0\}
$$

Es ist aber zu beachten, dass beim Modell "TLC" rund zweimal soviele Ungleichungen wie beim Modell "TLL" auftreten. 


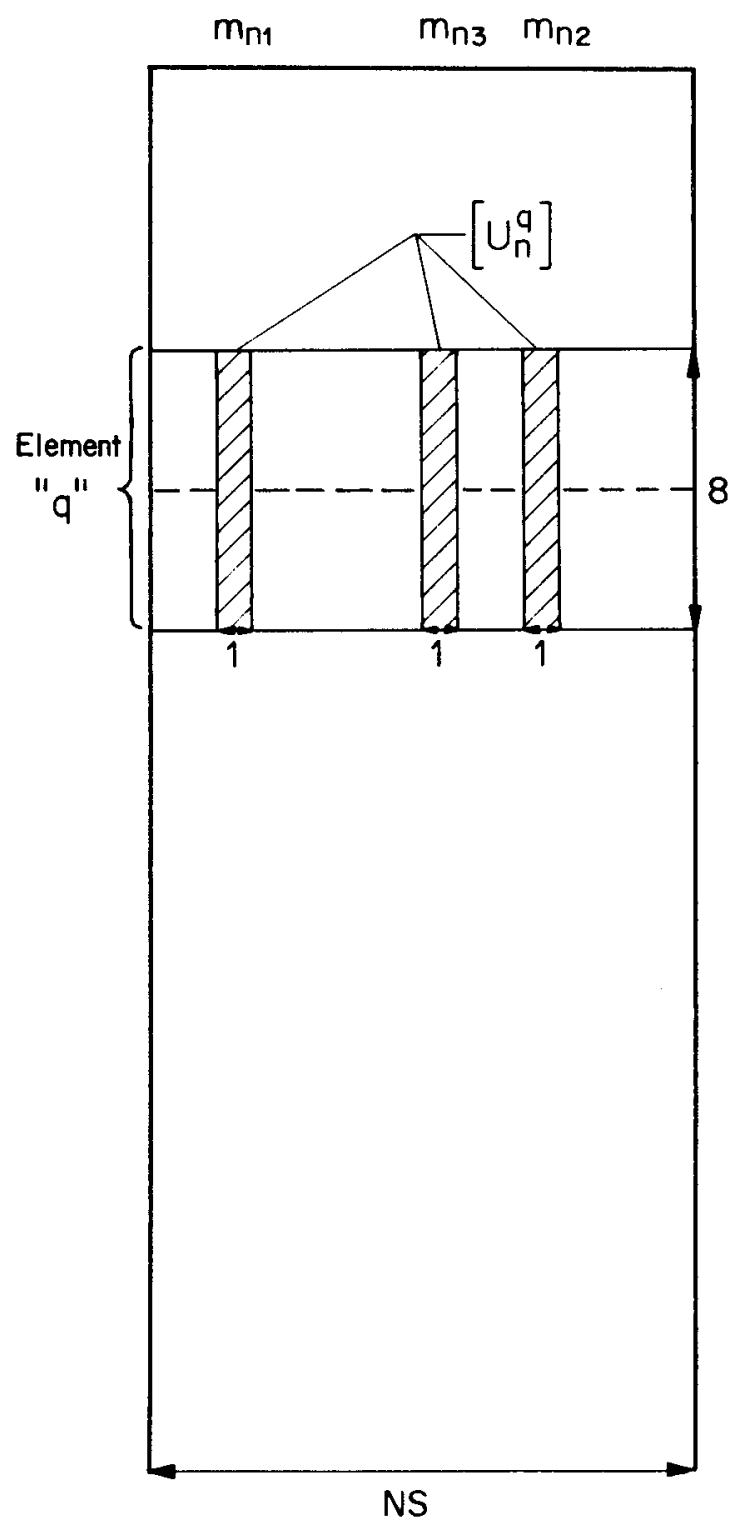

Tableau 5.2: Ungleichungsmatrix 


\subsection{Problemstellung}

Nach dem Satz über den untern Grenzwert (statischer Grenzwertsatz) ergeben alle Momentenzustände, die einerseits die Gleichgewichtsbedingungen

$$
[G]\{m\}-\lambda\{p\}=\{0\}
$$

und andererseits die Plastizitätsbedingungen

$$
[U]\{m\}+\{c\} \geqq\{0\}
$$

exakt erfüllen, einen untern Grenzwert für den Traglastfaktor $\lambda$. Wenn lokale Gleichgewichtsverletzungen auftreten, erhält man einen approximativen Wert für $\lambda$. Gesucht sind also das grösste $\lambda$ und der zugehörige Momentenzustand, welche die Bedingungen (4.28) und (4.31) erfüllen. Dieses Problem kann als lineares Programm (Tableau 6.1 und 6.2) dargestellt werden.

Die Untersuchung des dualen linearen Programmes lieferte bei ökonomischen und statischen Problemen interessante Resultate. So wurde zuerst für Stabtragwerke [24], dann für beliebige starr-plastische Körper [3], gezeigt, dass die Formulierung der Sätze über den obern und untern Grenzwert bei einer linearisierten Fliessfigur und einer endlichen Zahl von Spannungs- und Verschiebungsgeschwindigkeitsparametern auf zwei zueinander duale lineare Programme führt. Unter einem dualen Paar linearer Optimierungsprobleme versteht man die Aufgaben

$$
\begin{aligned}
& \lambda=0+\{d\}^{\top}\{x\} \rightarrow \operatorname{Mox} \\
& \{y\}=\{c\}+[A]\{x\} \geqq\{0\} \\
& \{x\} \geqq\{0\} \\
& \lambda=0+\{c\}^{\top}\{\bar{y}\} \rightarrow \operatorname{Min} \\
& \{\bar{x}\}=-\{d\}-\{A\}^{\top}\{\bar{y}\} \geqq\{0\} \\
& \{\bar{y}\} \geqq\{0\}
\end{aligned}
$$




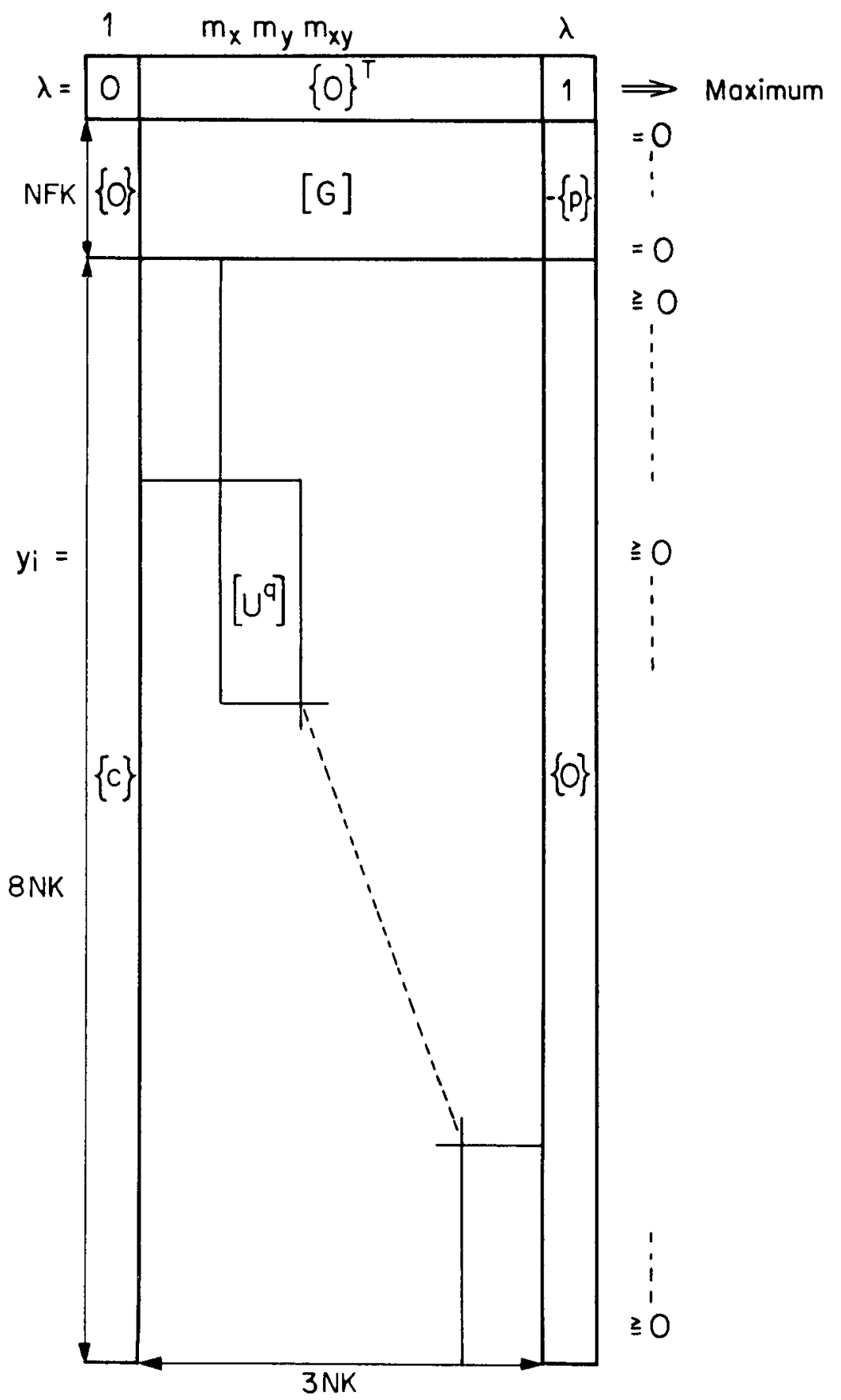

Tableau 6.1: Lineares Programm für das primale Problem (Modell mit lineoren Momenten) 


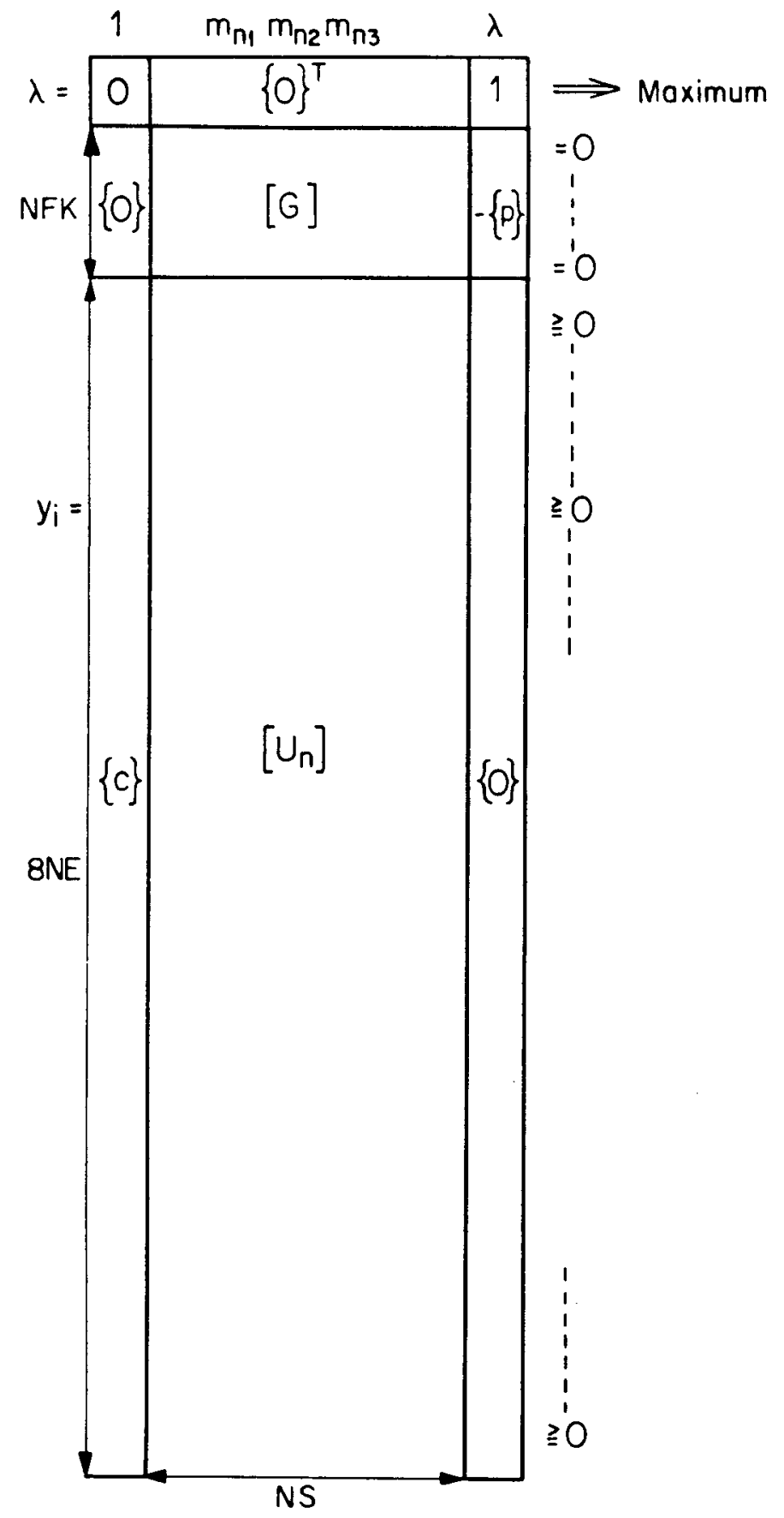

Tableau 6.2 : Lineares Programm für das primale Problem (Modell mit konstanten Momenten) 
Die entsprechenden zwei Aufgaben für unsere Traglastberechnung sind im Tableau 6.3 dargestellt. Die Komponenten des Vektors $\{m\}$ sind positiv oder negativ, diejenigen des Vektors $\{c\}$ sind positiv. Weil die komponenten von $\{m\}$ freie Variable sind und auch $\lambda$ nicht als positive Variable definiert wird, müssen im dualen Problem lauter Gleichungen auftreten.

Es stellt sich jetzt die Frage, welche physikalischen Grössen den unbekannten Variablen des dualen Problems entsprechen. Beim dualen Problem handelt es sich um die Traglastberechnung nach dem Satz über den obern Grenzwert (kinematischer Grenzwertsatz). Deshalb wird es sich um Beziehungen zwischen den kinematischen Grössen handeln.

\subsection{Kinematischer Aspekt der Fliessbedingung}

Man betrachtet einen Kontrollpunkt $q(x, y)$, in dem der Mamentenvektor eine Fliessfläche (Bild 2.6) erreicht hat. Nach der Theorie des plastischen Potentials steht dann der Vektor der Krümmungsgeschwindigkeiten senkrecht zur Fliessfläche und zeigt nach aussen. Dieser Zusammenhang ist im Bild 6.1 für vektoren mit zwei Komponenten $\left(m_{y}=0\right)$ aufgezeichnet.

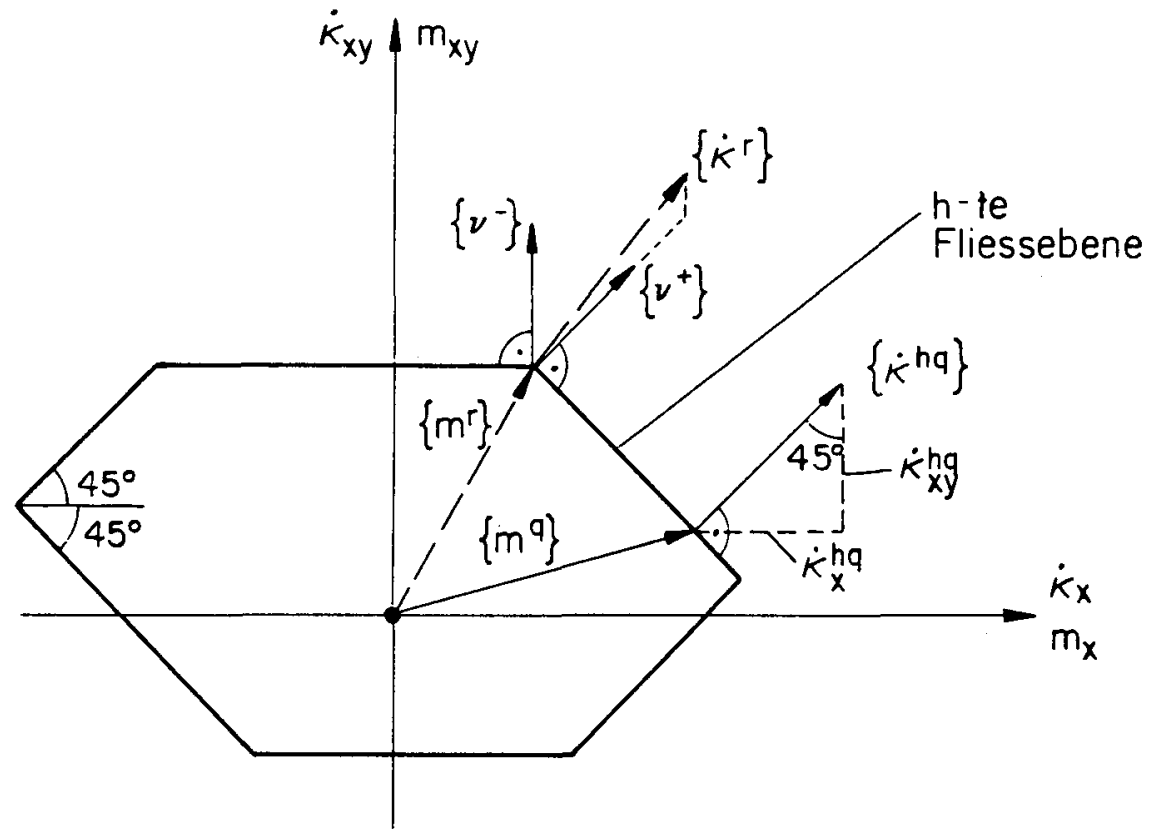

Bild 6.1: Kinematische Grössen aus Fliessfigur 

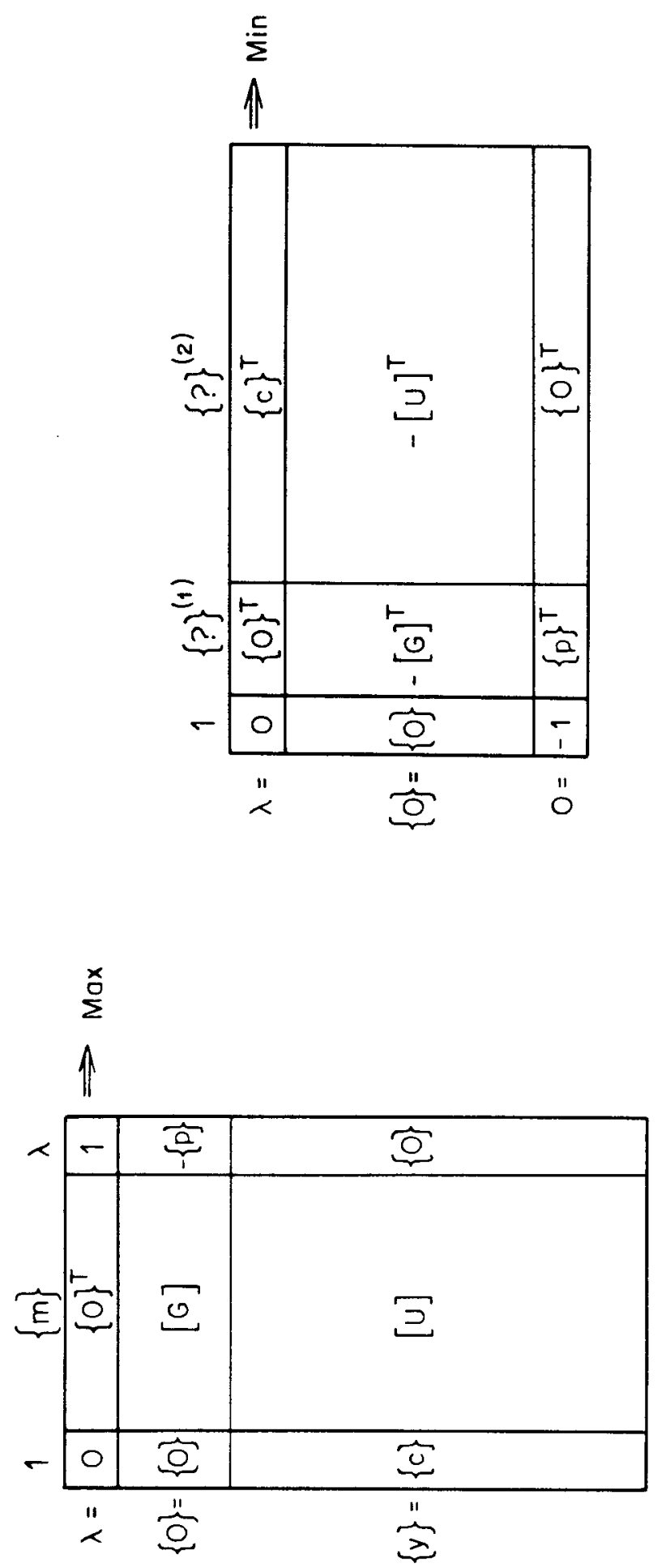

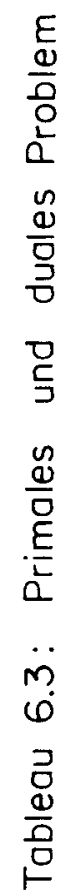


Wenn die Fliessfigur Knicke aufweist, ist die Richtung des Vektors $\left\{\dot{K}^{\Gamma}\right\}$ nicht bestimmt. Er kann eine beliebige lineare Kombination mit positiven Koeffizienten

$$
\left\{\dot{K}^{r}\right\}=a_{1}\left\{\nu^{-}\right\}+a_{2}\left\{\nu^{+}\right\} \quad\left(a_{1}, a_{2}>0\right)
$$

der angrenzenden Normalen sein.

Sowohl die nichtlineare Fliessbedingung (2.13), wie auch die linearisierte Fliessbedingung (2.14) stellen im euklidischen $\left(m_{x}-m_{y}{ }^{-m_{x y}}\right)$-Raum konvexe Körper dar. Damit gilt das Prinzip der grössten Dissipationsleistung (Bild 6.2), das heisst, der Vektor der krümmungsgeschwindigkeiten in einer

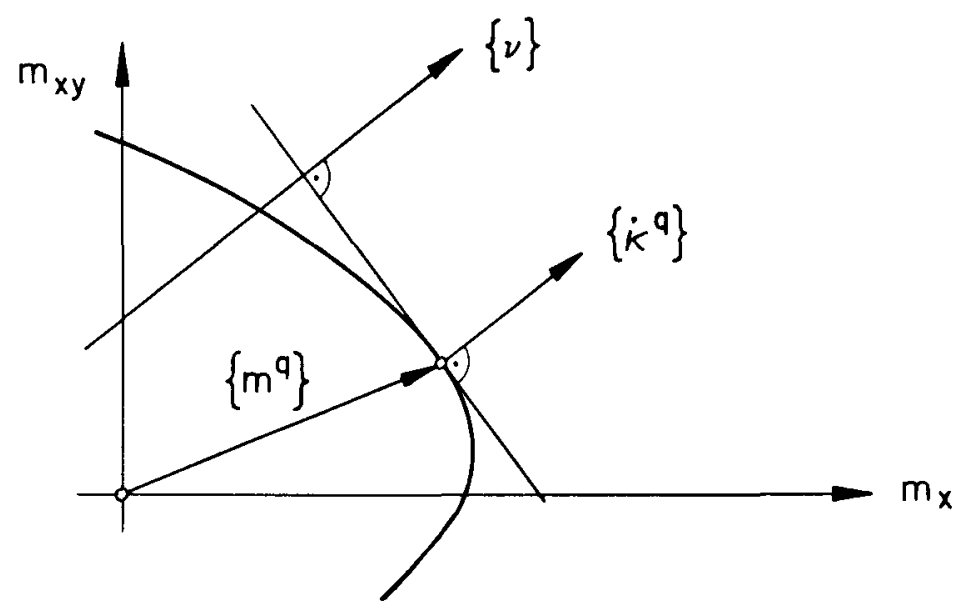

Bild 6.2: Orthogonalitäł des Vektors der Krümmungsgeschwindigkeiten

bestimmten Richtung $\{v\}$ ist demjenigen Vektor $\left\{m^{q}\right\}$ zugeordnet, der die grösste Dissipationsleistung

$$
d \dot{A}_{i}^{q}=\left\{m^{q}\right\}^{\top}\left\{\dot{k}^{q}\right\}
$$

erwirkt [25]. Es ist zu beachten, dass $\left\{\dot{k}^{q}\right\}$ nach dieser Bedingung zugeordnet wird und nicht nach der folgenden: Gesucht diejenige Richtung von $\left\{\dot{k}^{q}\right\}$, die bei gegebenem $\left\{m^{q}\right\}$ und gegebenem Betrag von $\left\{\dot{\kappa}^{q}\right\}$ die grösste Dissipationsleistung ergibt.

Gemäss Bild 6.1 sind die beiden Komponenten von $\left\{\dot{k}^{h q}\right\}$ gleich. Sie werden kurz mit

$$
\dot{\alpha}^{h q}=\dot{\kappa}_{x}^{h q}=\dot{K}_{x y}^{h q}
$$


bezeichnet. Wenn alle acht Fliessebenen der linearisierten Fliessfigur einbezogen werden, sind die Komponenten der Krümmungsgeschwindigkeit $\left\{\dot{K}^{\natural}\right\}$ im Punkt q

$$
\left\{\begin{array}{c}
\dot{K}_{x}^{q} \\
\dot{K}_{y}^{q} \\
\dot{K}_{x y}^{q}
\end{array}\right\}=\left[\begin{array}{cccccccc}
1 & 1 & 0 & 0 & -1 & -1 & 0 & 0 \\
0 & 0 & 1 & 1 & 0 & 0 & -1 & -1 \\
1 & -1 & 1 & -1 & 1 & -1 & 1 & -1
\end{array}\right]\left\{\begin{array}{c}
\dot{\alpha}^{1 q} \\
\dot{\alpha}^{2 q} \\
\dot{\alpha}^{3 q} \\
\dot{\alpha}^{4 q} \\
\dot{\alpha}^{5 q} \\
\dot{\alpha}^{6 q} \\
\dot{\alpha}^{7 q} \\
\dot{\alpha}^{8 q}
\end{array}\right\}
$$

Dabei ist $\dot{\alpha}^{\text {hq }} \neq 0$, wenn $\left\{\mathrm{m}^{q}\right\}$ die h-te Fliessebene erreicht. Die Gleichung (6.6) kann mit der Definition (2.16) geschrieben werden als

$$
\left\{\dot{K}^{q}\right\}=-\left[U^{q}\right]^{T}\left\{\dot{\alpha}^{q}\right\}
$$

\subsection{Kontrolle des Mechanismus}

Das Geschwindigkeitsfeld im Kollapszustand (Mechanismus) wird durch den stückweise linearen Ansatz

$$
\dot{w}(x, y)=\left\langle\dot{w}^{i} \quad \dot{w}^{j} \quad \dot{w}^{k}\right\rangle \quad\left\{\begin{array}{l}
a_{1} \\
a_{2} \\
a_{3}
\end{array}\right\}
$$

approximiert. Die Punkte bedeuten Ableitungen nach der Zeit; die Bezeichnungen entsprechen im übrigen denjenigen in der Gleichung (4.1). Das Geschwindigkeitsfeld in der Umgebung des Knotens $i$ ist im Bild 6.3 dargestellt. 

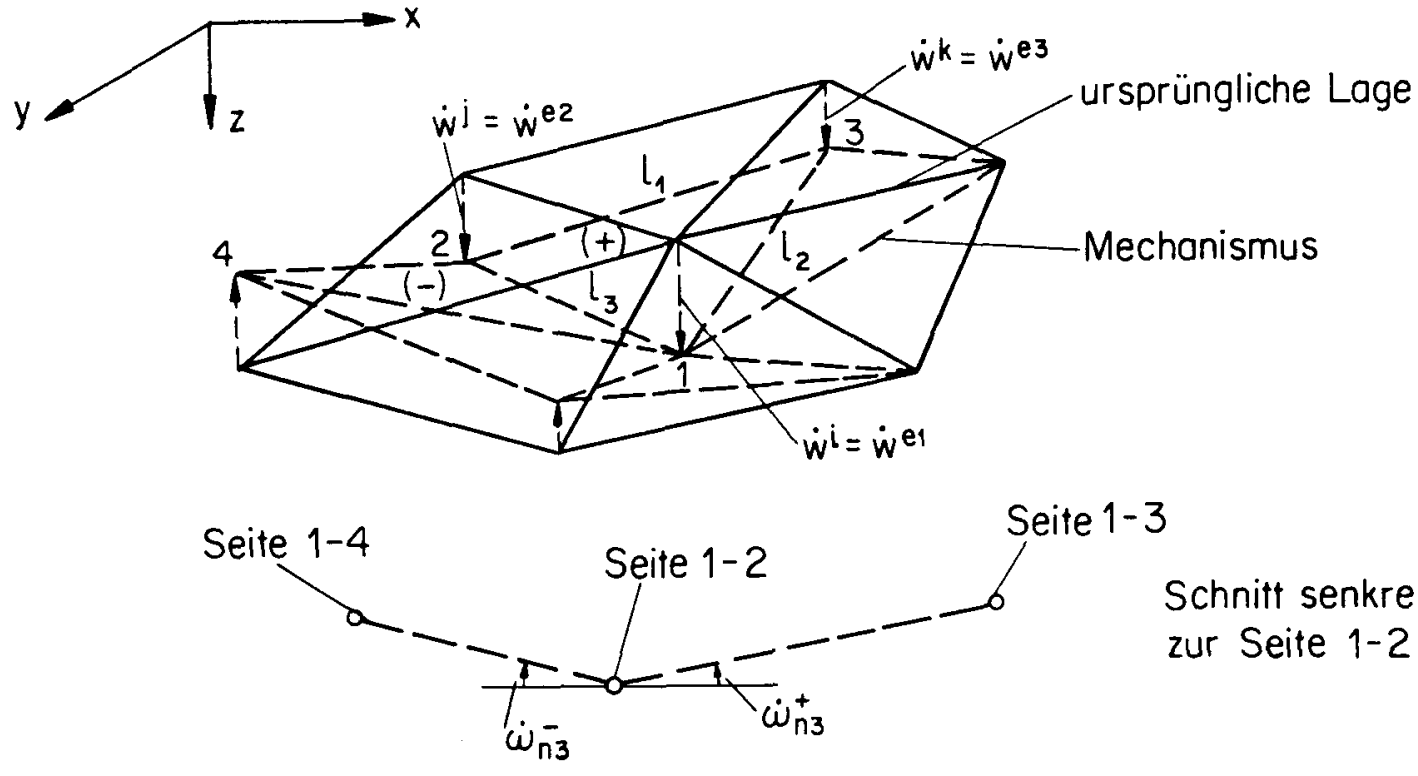

Schnitt senkrecht zur Seite 1-2

\section{Bild 6.3: Geschwindigkeitsfeld}

Dieses Geschwindigkeitsfeld muss mit den Krümmungsgeschwindigkeiten aus der Fliessfigur verträglich sein.

Es ist zweckmässig, für diese Kompatibilitätskontrolle das Prinzip der virtuellen Spannungen zu benutzen. Die Kompatibilitätsbedingungen für Platten sind erfültt, wenn für jedes virtuelle Momentenfeld $m_{i j}^{*}(x, y)$, welches die statischen Randbedingungen erfüllt,

$$
\iint_{F} m_{i j}^{*}(x, y) \dot{w}_{i j}(x, y) d F+\iint_{F} m_{i j}^{*} \dot{\kappa}_{i j}(x, y) d F=0
$$

Die Bezeichnungen sind im Abschnitt 3.1 definiert. Die Krümmungsgeschwindigkeiten $\dot{k}_{i j}$ sind der Fliessfigur, die partiellen Ableitungen $\dot{w}_{\text {, ij }}$ sind dem Mechanismus zu entnehmen. Man setzt die Näherungsfunktionen (3.1) und (3.2) ein und erhält

$$
\iint_{F} m_{r}^{*} \psi_{r i j} \varphi_{m, i j} \dot{w}_{m} d F+\iint_{F} m_{r}^{*} \psi_{r i j} \dot{\kappa}_{i j} d F=0
$$

Die Kompatibilitätsbedingungen werden nicht mehr für alle $m_{i j}^{*}(x, y)$ kontrolliert, sondern nur noch für die virtuellen Momentenfelder, die sich aus der Variation der Momentenparameter $m_{r}(r=1, R)$ ergeben. Für jedes virtuelle Moment $m_{r}^{*}$ erhalten wir eine Kompatibilitätsgleichung 


$$
m_{r}^{*}\left[\left(\iint_{F} \psi_{r i j} \varphi_{m, i j} d F\right) \dot{w}_{m}+\iint_{F} \psi_{r i j} \dot{\kappa}_{i j} d F\right]=0
$$

Wie ein Vergleich mit den Gleichungen (3.13) und (3.15) zeigt, besteht das erste Integral aus den Koeffizienten

$$
\iint_{F} \psi_{r i j} \varphi_{m, i j} d F=-g_{r m}
$$

Diese Koeffizienten können bei den verwendeten Modellen auch auf einfache Weise geometrisch bestimmt werden (Bild 6.3). Das zweite Integral der Gleichung (6.11) ist schwierig auszuwerten, weil die fliessbedingung nur an den Q Kontrollpunkten definiert ist. Das Integral kann nicht ohne zusätzliche Annahmen über den Verlauf von $\dot{k}_{i j}(x, y)$ berechnet werden. Man kann eine "konzentrierte krümmungsgeschwindigkeit" $\dot{k}_{i j}^{q}$ im Kontrollpunkt einführen (ähnlich wie man verteilte Lasten durch Knotenlasten approximiert). Damit wird das zweite Integral zu

$$
\iint_{F} \psi_{\text {rij }} \dot{K}_{i j} d F=\sum_{q} \psi_{r i j}^{q} \dot{k}_{i j}^{q}=\sum_{q}\left[\psi^{q}\right]^{\top}\left\{\dot{k}^{q}\right\}
$$

Die Gleichung (6.7) kann auch für $\dot{k}_{i j}^{q}$ geschrieben werden

$$
\left\{\dot{k}^{q}\right\}=-\left[U^{q}\right]^{\top}\left\{\dot{\beta}^{q}\right\}
$$

Der Ausdruck (6.13) wird dann zu

$$
\sum_{q}\left[\psi^{q}\right]^{\top}\left\{\dot{k}^{q}\right\}=-\sum_{q}\left[\psi^{q}\right]^{\top}\left[U^{q}\right]^{\top}\left\{\dot{\beta}^{q}\right\}
$$

Wenn ein Vektor $\{\dot{\beta}\}$, der sich aus den Subvektoren $\left\{\dot{\beta}^{q}\right\}(q=1, Q)$ zusammensetzt, und die im Tableau 3.1 dargestellte Matrix [U] eingeführt werden, so kann der Ausdruck (6.15) geschrieben werden als

$$
-\sum_{q}\left[\psi^{q}\right]^{\top}\left[U^{q}\right]^{\top}\left\{\dot{\beta}^{q}\right\}=-[U]^{\top}\{\dot{\beta}\}
$$


Die Gleichungen (6.16) und (6.12) werden in die Gleichung (6.11) eingesetzt.

$$
-[G]^{\top}\{\dot{w}\}-[U]^{\top}\{\dot{\beta}\}=\{0\}
$$

In diesem Gleichungssystem sind alle R Kompatibilitätsbedingungen enthalten.

Für den Spezialfall, dass die virtuellen Momente elementweise konstant sind, lässt sich das Integral in der Gleichung (6.13) noch auf eine andere weise vereinfachen:

$$
\begin{aligned}
& \iint_{F} \psi_{\text {rij }} \dot{\kappa}_{i j} d F=\sum_{e} \psi_{\text {rij }}^{e} \iint_{F^{e}} \dot{K}_{i j} d F \\
& =-\sum_{e}\left[\psi^{e}\right]^{T}\left[U^{e}\right]^{\top} \iint_{F^{e}}\{\dot{\alpha}\} d F
\end{aligned}
$$

In diesem Fall ist also $\left\{\dot{\beta}^{q}\right\}$ definiert als

$$
\left\{\dot{\beta}^{q}\right\}=\left\{\dot{\beta}^{e}\right\}=\iint_{F^{e}}\{\dot{\alpha}\} d F
$$

Nun werden die Kompatibilitätsbedingungen für die beiden Modelle "TLL" und "TLC", die in den Kapiteln 4 und 5 verwendet wurden, formuliert.

Zuerst sei das Modell "TLC" untersucht. Das entsprechende duale Problem (Tableau 6.3) enthält pro Seite eine kinematische Kontrolle, weil die Matrizen [G] ${ }^{\top}$ und $[U]^{\top}$ NS Zeilen aufweisen. Es ist also zu erwarten, dass die Rotationsgeschwindigkeiten $\Delta \dot{\omega}_{n}=\dot{\omega}_{n}^{-}+\dot{\omega}_{n}^{+}$entlang den Seiten kontrolliert werden.

Das Element $1,2,3$ im Bild 6.3 hat die Seitenlängen $l_{1}, l_{2}$ und $l_{3}$. Die Geschwindigkeiten $\dot{\omega}_{n}$ lassen sich gemäss Gleichung (4.7) wie folgt in Funktion der Verschiebungsgeschwindigkeiten der Knoten ausdrücken

$$
\begin{aligned}
& \left\{\begin{array}{c}
\dot{\omega}_{n 1} l_{1} \\
\dot{\omega}_{n 2} l_{2} \\
\dot{\omega}_{n 3} l_{3}
\end{array}\right\}=\left[\begin{array}{ccc}
-\left(c_{2}+c_{3}\right) & c_{3} & c_{2} \\
c_{3} & -\left(c_{3}+c_{1}\right) & c_{1} \\
c_{2} & c_{1} & -\left(c_{1}+c_{2}\right)
\end{array}\right]\left\{\begin{array}{l}
\dot{w}^{1} \\
\dot{w}^{2} \\
\dot{w}^{3}
\end{array}\right\} \\
& \text { oder : } \quad\left\{\dot{w}_{n}^{e} l^{e}\right\}=\left[Q^{e}\right]\left\{\dot{w}^{e}\right\}
\end{aligned}
$$


Dabei folgen $c_{d}=\cot \vartheta_{d}(d=1,2,3)$ aus Bild 4.7 .

Man führt nun die Summation über alle Elemente durch und erhält

$$
\left\{\Delta \dot{\omega}_{n} l\right\}=\sum_{e}\left[L_{n}^{e}\right]^{T}\left[Q^{e}\right]\left\{\dot{w}^{e}\right\}=\left\{\dot{k}_{n}\right\}
$$

Die Definition der Matrizen $\left[L_{n}^{e}\right]$ ist im Bild 4.8 angegeben. Der Vektor $\left\{\Delta \dot{\omega}_{n} l\right\}$ hat NS Komponenten und wird abgekürzt als $\left[\dot{k}_{n}\right]$ bezeichnet, weil er die Dimension einer konzentrierten Krümmungsgeschwindigkeit hat. Unter Berücksichtigung der Gleichung (4.14) ergibt sich

$$
\left\{\dot{k}_{n}\right\}=\sum_{e}\left[L_{n}^{e}\right]^{\top}\left[Q^{e}\right]\left[L_{w}^{e}\right]\{\dot{w}\}
$$

Wenn man schliesslich die Gleichung (5.8) einsetzt und berücksichtigt, dass nur freie knoten eine Verschiebungsgeschwindigkeit haben können, bekommt man die "duale Beziehung" zur Gleichung (4.28)

$$
\left\{\dot{k}_{n}\right\}=[G]^{\top}\{\dot{w}\}
$$

Die Komponenten des Vektors $\left\{\dot{\Delta}_{n} l\right\}$ können andererseits aus der Gleichung (6.7) berechnet werden. Zur Berechnung der Komponente $\Delta_{*} \dot{\omega}_{n 1}{ }^{l_{1}}$ bringen wir ein virtuelles Momentenfeld mit $m_{n 1}^{*} \neq 0\left(m_{n 2}^{*}=m_{n 3}^{*}=0\right)$ an.

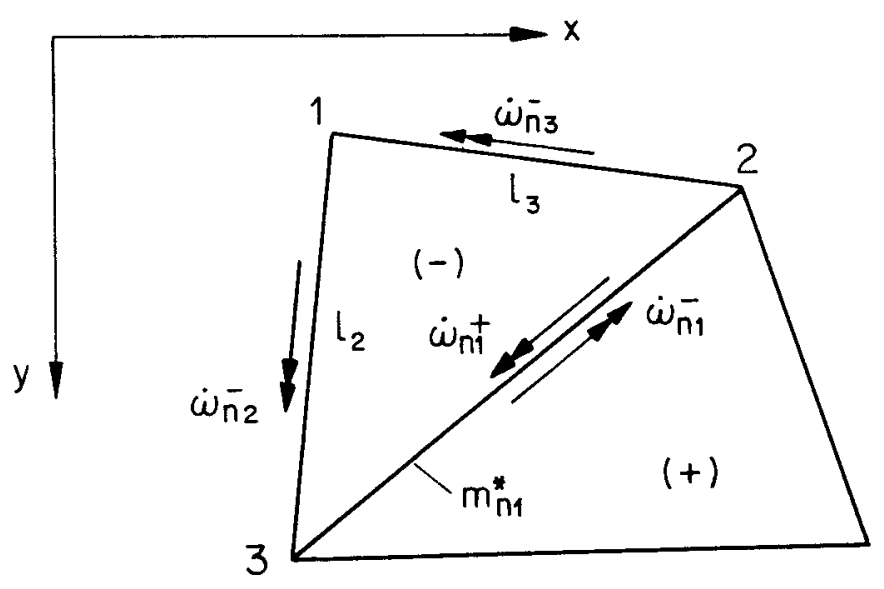

Bild 6.4: Berechnung von $\Delta \dot{\omega}_{n 1} l_{1}$ 
Die Momente in einem Element sind nach Gleichung (5.2)

$$
\left\{m_{x-y}^{* e}\right\}^{(1)}=\left[T_{n}\right]^{-1}\left\{m_{n}^{* e}\right\}^{(1)}
$$

Die äussere und innere Leistung der Krümmungsgeschwindigkeiten für ein Element infolge des virtuellen Momentenfeldes sind

$$
\begin{aligned}
& \dot{A}_{0}^{* e}=-\left\{\dot{\omega}_{n} l\right\}^{\top}\left\{m_{n}^{* e}\right\}^{(1)}=-\dot{\omega}_{n 1}^{e} l_{1} m_{n 1}^{*} \\
& \dot{A}_{i}^{* e}=\iint_{F^{e}}\left\{\dot{K}^{q}\right\}^{\top}\left\{m_{x-y}^{* e}\right\}^{(1)} d x d y
\end{aligned}
$$

Alle Komponenten von $\left\{m_{x-y}^{*}{ }^{*}\right\}$ sind über den Bereich $F^{e}$ eines Elementes konstant. Da die Beanspruchung $\left\{\mathrm{m}_{x-y}^{e}\right\}$ ebenfalls als konstant vorausgesetzt wurde (Kapitel 5), wird angenommen, dass auch $\left\{\dot{K}^{q}\right\}$ über den Bereich $F^{e}$ konstant ist. Ferner wird die Gleichung (6.23) eingesetzt.

$$
\dot{A}_{i}^{* e}=\left\{\dot{\kappa}^{e}\right\}^{\top}\left[T_{n}\right]^{-1}\left\{m_{n}^{* e}\right\}^{(1)} F^{e}
$$

Die Summation über alle Elemente ergibt für die innere und äussere Leistung

$$
\begin{aligned}
& \dot{A}_{0}^{*}=-\sum_{e}\left\{\dot{\omega}_{n} l\right\}^{\top}\left[L_{n}^{e}\right] \cdot\left\{m_{n}^{*}\right\}^{(1)}=-\Delta \dot{\omega}_{n 1} l_{1} m_{n 1}^{*} \\
& \dot{A}_{i}^{*}=\sum_{e}\left\{\dot{K}^{e}\right\}^{\top}\left[T_{n}\right]^{-1}\left[L_{n}^{e}\right] F^{e} \cdot\left\{m_{n}^{*}\right\}^{(1)}
\end{aligned}
$$

Wie bei der Gleichung (6.20) führt man konzentrierte Krümmungsgeschwindigkeiten ein.

$$
\left\{\dot{k}_{n}\right\}=\left\{\Delta \dot{\omega}_{n} l\right\}=\left(\sum_{e}\left\{\dot{\omega}_{n} l\right\}^{\top}\left[L_{n}^{e}\right]\right)^{\top}
$$

Aus der Bedingung $\dot{A}_{a}^{*}+\dot{A}_{i}^{*}=0$ und der Gleichung (6.26) ergibt sich dann

$$
\dot{k}_{n 1} m_{n 1}^{*}=\sum_{e}\left\{\dot{\kappa}^{e}\right\}^{\top}\left[T_{n}\right]^{-1}\left[L_{n}^{e}\right] F^{e}\left\{m_{n}^{*}\right\}^{(1)}
$$


Die übrigen Komponenten von $\left\{\dot{k}_{n}\right\}$ können auf dieselbe Weise berechnet werden.

$$
\left\{\dot{k}_{n}\right\}=\sum_{e}\left[L_{n}^{e}\right]^{\top}\left[T_{n}^{-1}\right]^{\top}\left\{\dot{\kappa}^{e}\right\} F^{e}
$$

Nun wird die Beziehung (6.7) eingesetzt.

$$
\left\{\dot{k}_{n}\right\}=-\sum_{e}\left[L_{n}^{e}\right]^{\top}\left[T_{n}^{-1}\right]^{\top}\left[U^{e}\right]^{\top}\left\{\dot{\alpha}^{e}\right\} F^{e}
$$

Der in der Gleichung $(6.18)$ definierte Vektor $\left\{\dot{\beta}^{q}\right\}$ wird für diesen Spezialfall

$$
\left\{\dot{\beta}^{q}\right\}=\left\{\dot{\alpha}^{e}\right\} \quad F^{e}
$$

Der lokale Vektor $\left\{\dot{\beta}^{q}\right\}$ kann mittels der Matrix $\left[L_{q}^{e}\right]$ aus der Gleichung (5.12) einem globalen Vektor $\{\dot{\beta}\}$ zugeordnet werden

$$
\left\{\dot{\beta}^{q}\right\}_{8 \times 1}=\left[L_{q}^{e}\right]^{\top}\{\dot{\beta}\}_{8 N E \times 1}
$$

Damit erhält man aus der Gleichung (6.29)

$$
\left\{\dot{k}_{n}\right\}=-\sum_{e}\left(\left[L_{n}^{e}\right]^{\top}\left[T_{n}^{-1}\right]^{\top}\left[U^{e}\right]^{\top}\left[L_{q}^{e}\right]^{\top}\right)\{\dot{\beta}\}
$$

Der Vergleich mit den Gleichungen (5.11) und (5.12) ergibt

$$
\left\{\dot{k}_{n}\right\}=-[U]^{\top}\{\dot{\beta}\}
$$

Das ist die "duale Beziehung" zur Gleichung (4.31).

Wenn für einen Parameter eine statische Randbedingung existiert, fällt eine der Kompatibilitätsbedingungen (6.11) weg, weil ein zugehöriges virtuelles Moment $m_{r}^{*} \neq 0$ die statische Randbedingung verletzen würde. Die statischen Randbedingungen haben zur Folge, dass die Komponente von $\left\{\dot{k}^{q}\right\}$ in Richtung der zugehörigen Koordinatenaxe keinen Beitrag zur Dissipationsleistung ergibt. Diese Komponente (z.B. $\dot{k}_{y}$ im Bild 6.1) ist unbestimmt und 
die entsprechende Zeile in der Gleichung (6.33) fällt weg.

Die Gleichungen (6.33) und (6.22) bilden die gesuchte Beziehung zwischen den Kinematischen Grössen (Kompatibilitätsbedingung)

$$
-[G]^{\top}\{\dot{w}\}=[U]^{\top}\{\dot{\beta}\}=\{0\}
$$

Damit ist die physikalische Bedeutung der Vektoren $\{\text { ? }\}^{(1)}$ und $\{\text { ? }\}^{(2)}$ im Tableau 6.3 gefunden: $\{\text { ? }\}^{(1)}$ ist der Vektor $\{\dot{w}\}$ der vertikalen Verschiebungsgeschwindigkeiten und $\{\text { ? }\}^{(2)}$ ist der Vektor $\{\dot{\beta}\}$ der konzentrierten Krümmungsgeschwindigkeitskomponenten pro Element und Fliessebene. Die $\dot{w}^{i}$ sind freie Variable, die $\dot{\beta}^{j}$ sind nichtnegativ.

Es ist zu beachten, dass die Kompatibilitätskontrolle nicht vollständig ist. Es wird lediglich kontrolliert, ob die relative Rotationsgeschwindigkeit $\Delta \dot{\omega}_{n}=\dot{\omega}_{n}^{-}+\dot{\omega}_{n}^{+}$verträglich ist. Der resultierende Traglastfaktor $\lambda$ ist kein oberer Grenzwert.

Beim zweiten Modell, dem Modell "TLL", werden die konzentrierten Krümmungsgeschwindigkeiten in den knoten kontrolliert. Durch die Matrix [G] wurde der Zusammenhang zwischen den resultierenden Knotenkräften $\{p\}$ und den Momenten in den knoten hergestellt, indem man virtuelle verschiebungsfelder $w^{*} i$ anbrachte. Durch [G] ${ }^{\top}$ wird nun der Zusammenhang zwischen den resultierenden (in den knoten konzentrierten) Krümmungsgeschwindigkeiten und den Geschwindigkeiten der knotenverschiebungen $\{\dot{w}\}$ hergestellt, indem man virtuelle Momentenfelder ${ }^{*} i$ anbringt.

Zuerst werden die konzentrierten Krümmungsgeschwindigkeiten in den knoten aus den vertikalen Verschiebungsgeschwindigkeiten berechnet. Beim Modell "TLC" wurde dieser Zusammenhang geometrisch ermittelt. Hier soll das generelle Verfahren, das Prinzip der virtuellen Spannungen, angewendet werden. Die innere und äussere Leistung der Krümmungsgeschwindigkeiten infolge des virtuelien Momentenfeldes $m_{x}^{* 1}$ (Bild 6.5)

$$
\left\{m_{x-y}^{* e}\right\}^{(1, x)}=\left\{\begin{array}{c}
m_{x}^{* 1} \\
0 \\
0 \\
\cdots \\
0 \\
0 \\
0 \\
\cdots \\
0 \\
0 \\
0
\end{array}\right\}
$$




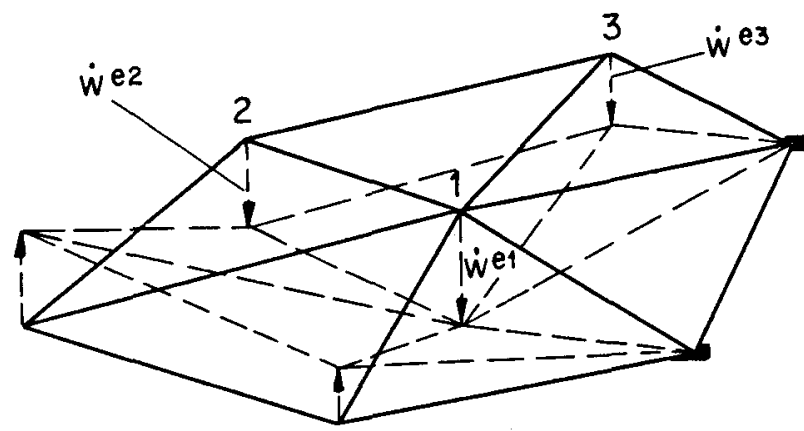

Geschwindigkeitsfeld $\dot{w}(x, y)$

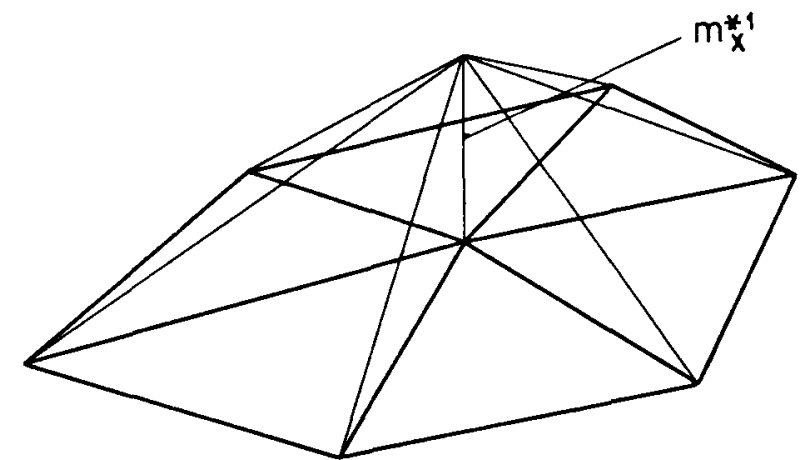

virtuelles

Momentenfeld

$m_{x}^{* 1}(x, y)$

Bild 6.5: Anwendung des Prinzipes der virtuellen Spannungen

für ein Element sind zum Beispiel

$$
\begin{aligned}
& \dot{A}_{0}^{* e}=-\left\{\dot{k}^{e}\right\}^{\top}\left\{m_{x-y}^{* e}\right\}^{(1, x)}=-\dot{k}_{x}^{1 e} \cdot m_{x}^{* 1} \\
& \dot{A}_{i}^{* e}=\iint_{\dot{F}^{e}}\left\{\dot{K}^{e}\right\}^{\top}\left\{m_{x-y}^{* e}\right\}^{(1, x)} d x d y
\end{aligned}
$$

Krümmungsgeschwindigkeiten treten beim Geschwindigkeitsfeld nach Gleichung (6.8) nur entlang den Seiten auf und sind in der Gleichung (6.19) angegeben. Die Momente entlang den Seiten berechnen sich aus Gleichung (6.35) mittels der Gleichung (4.8). Der Beitrag eines Elementes ist

$$
\begin{aligned}
\dot{A}_{i}^{* e} & =\left\{\dot{\omega}_{n}^{e} \cdot l\right\}^{\top}\left\{m_{n}^{* e}\right\}^{(1, x)} \\
& =\left\{\dot{w}^{e}\right\}^{\top}\left[Q^{e}\right]^{\top}\left[T_{x-y}^{e}\right] \quad\left\{m_{x-y}^{* e}\right\}^{(1, x)}
\end{aligned}
$$

Die Summation über alle Elemente erfolgt wie bei der Herleitung der Gleichung (4.16). 


$$
\dot{A}_{i}^{*}=\sum_{e}\{\dot{w}\}^{\top}\left[L_{w}^{e}\right]^{\top}\left[Q^{e}\right]^{\top}\left[T_{x-y}^{e}\right]\left[L_{m}^{e}\right]\left\{m_{x-y}^{*}\right\}^{(4, x)}
$$

Nach dem Prinzip der virtuellen Spannungen ist $\dot{A}_{a}^{*}+\dot{A}_{i}^{*}=0$ und damit

$$
\dot{k}_{x}^{\prime} m_{x}^{* 1}=\sum_{e}\{\dot{w}\}^{\top}\left[L_{w}^{e}\right]^{\top}\left[Q^{e}\right]^{\top}\left[T_{x-y}^{e}\right]\left[L_{m}^{e}\right]\left\{m_{x-y}^{*}\right\}^{(1, x)}
$$

Auch die übrigen Komponenten der konzentrierten Krümmungsgeschwindigkeiten lassen sich durch Anbringen entsprechender virtueller Momentenfelder berechinen.

$$
\{\dot{k}\}=\sum_{e}\left[L_{m}^{e}\right]^{\top}\left[T_{x-y}^{e}\right]^{\top}\left[Q^{e}\right]\left[L_{w}^{e}\right]\{\dot{w}\}
$$

Aus dem Vergleich mit der Gleichung (4.16) bekommt man schliesslich die "duale Beziehung" zur Gieichung (4.28)

$$
\{\dot{k}\}=[G]^{\top}\{\dot{w}\}
$$

Auf der anderen Seite sollten die konzentrierten Krümmungsgeschwindigkeiten in den Knoten aus der Gleichung (6.7) berechnet werden. Die Krümmungsgeschwindigkeiten aus der Fliessfigur können aber nur in den Knoten angegeben werden. Eine Integration des zweiten Integrals in der Gleichung (6.11) ist deshalb nicht möglich. Man kann im Bild 6.1 anstelle der Krümmungsgeschwindigkeiten die konzentrierten Krümmungsgeschwindigkeiten einzeichnen und er$h \ddot{E} I t$

$$
\left\{\dot{k}^{q}\right\}=-\left[U^{q}\right]^{\top}\left\{\dot{\beta}^{q}\right\}
$$

Der lokele Vektor $\left\{\dot{\beta}^{q}\right\}$ kann einem globalen Vektor $\{\dot{\beta}\}$ mittels $\left[L_{q}^{j}\right]$ aus der Gleichung (4.30) zugeordnet werden

$$
\left\{\dot{\beta}^{q}\right\}_{8 \times 1}=\left[L_{q}^{j}\right]^{\top}\{\dot{\beta}\}_{8 N K \times 1}
$$


Damit wird aus der Gleichung (6.14) für den Knoten $j=q$

$$
\left\{\dot{k}^{q}\right\}=-\left[U^{q}\right]^{\top}\left[L_{q}^{j}\right]^{\top}\{\dot{\beta}\}
$$

Der Vektor $\left\{\dot{k}^{q}\right\}$ kann mittels $\left[L_{m}^{j}\right]$ aus der Gleichung (4.29) einem globalen Vektor $\{\dot{k}\}$ zugeordnet werden

$$
\{\dot{k}\}=\sum_{j}\left[L_{m}^{j}\right]^{\top} \quad\left\{\dot{k}^{q}\right\}
$$

womit schliesslich

$$
\{\dot{k}\}=-\sum_{j}\left(\left[L_{m}^{j}\right]^{\top}\left[U^{q}\right]^{\top}\left[L_{q}^{j}\right]^{\top}\right)\{\dot{\beta}\}
$$

Der Vergleich mit der Gleichung (4.30) ergibt

$$
\{\dot{k}\}=-[U]^{\top}\{\dot{\beta}\}
$$

Die Gleichungen (6.43) und (6.39) bilden die gesuchte Beziehung (6.22) zwischen den kinematischen Grössen (Kompatibilitätsbedingung)

$$
-[G]^{\top}\{\dot{w}\}-[U]^{\top}\{\dot{\beta}\}=\{0\}
$$

Man stellt auch hier fest, dass die Kontrolle nicht vollständig ist. Es wird lediglich verlangt, dass die "Resultierenden" der Krümmungsgeschwindigkeiten entlang den Seiten (die konzentrierten Krümmungsgeschwindigkeiten pro knoten) die Bedingungen erfüllen, die sich aus der Fliessbedingung ergeben.

Die physikalische Bedeutung der Vektoren $\{\text { ? }\}^{(1)}$ und $\{\text { ? }\}^{(2)}$ im Tableau 6.3 ist für das Modell "TLL" die folgende: \{?\} $(1)$ ist der Vektor $\{\dot{w}\}$ der vertikalen Verschiebungsgeschwindigkeiten, \{\}$^{(2)}$ ist der Vektor $\{\dot{\beta}\}$ der konzentrierten Krümmungsgeschwindigkeitskomponenten pro Knoten und Fliessebene. Die $\dot{w}^{i}$ sind freie Variable; die $\dot{\beta}^{j}$ sind nichtnegative Variable. 
Die Leistung der Knotenlasten $\lambda\{p\}$ für das in der Gleichung (6.8) definierte Geschwindigkeitsfeld im Kollapszustand ist

$$
\dot{A}_{0}=-\lambda\{p\}^{\top}\{\dot{w}\}
$$

Die Dissipationsleistung ist allgemein

$$
\dot{A}_{i}=\iint_{F}\{\dot{K}\}^{\top}\left\{m_{x-y}\right\} d x d y
$$

wobei

$$
\{\dot{k}\}=\left\{\begin{array}{c}
\dot{\kappa}_{x} \\
\dot{k}_{y} \\
\dot{k}_{x y}
\end{array}\right\} \quad\left\{m_{x-y}\right\}=\left\{\begin{array}{l}
m_{x} \\
m_{y} \\
m_{x y}
\end{array}\right\}
$$

Ortsfunktionen im Koordinatensystem $(x-y)$ sind.

Zur Berechnung von $\left\{\dot{k}^{q}\right\}^{\top}\left\{m_{x-y}^{q}\right\}$ nimmt man z.B. an, der Momentenvektor habe die h-te Fliessebene erreicht.

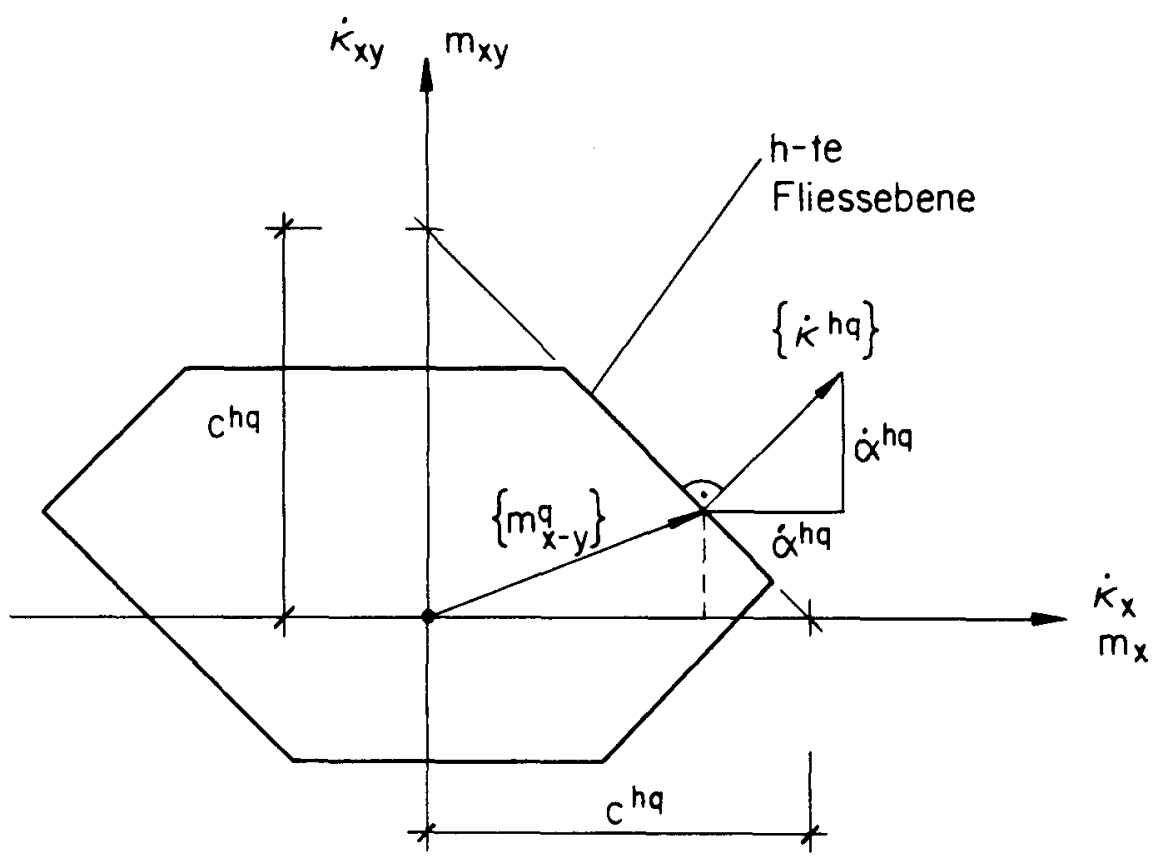

Bild 6.6: Berechnung der Dissipationsleistung 
In diesem Fall wird die h-te Zeile der Gleichung (2.15) zu

$$
c^{h q}=-\left\{u^{h q}\right\}^{\top}\left\{m_{x-y}^{q}\right\}
$$

Andererseits ist nach der Gleichung (6.7)

$$
\left\{\dot{\kappa}^{h q}\right\}=-\left\{u^{h q}\right\} \dot{\alpha}^{h q}
$$

Damit ist die Dissipationsleistung im Punkt q pro Flächeneinheit

$$
\begin{aligned}
d \dot{A}_{i}^{h q} & =\left\{\dot{\kappa}^{h q}\right\}^{\top}\left\{m_{x-y}^{q}\right\} \\
& =-\left\{u^{h q}\right\}^{\top}\left\{m_{x-y}^{q}\right\} \dot{\alpha}^{h q}=c^{h q} \cdot \dot{\alpha}^{h q}
\end{aligned}
$$

Wenn man z.B. als Kontrolle die erste Fliessebene betrachtet, ist aus dem Bild 6.6 leicht ersichtlich, dass

$$
\begin{aligned}
d \dot{A}_{i}^{q q} & =m_{x}^{q} \dot{\kappa}_{x}^{1 q}+m_{x y}^{q} \dot{\kappa}_{x y}^{q q} \\
& =\dot{\alpha}^{q q}\left(m_{x}^{q}+m_{x y}^{q}\right)=\dot{\alpha}^{1 q} c^{q q}
\end{aligned}
$$

Bezieht man alle Fliessebenen ein, so wird

$$
d \dot{A}_{i}^{q}=\left\{\dot{\alpha}^{q}\right\}^{\top} \quad\left\{c^{q}\right\}
$$

Die Integration der Gleichung (6.45) wurde im letzten Abschnitt bereits durchgeführt. Im folgenden wird das Resultat für das Modeli "TLC" angegeben; die Untersuchung des Modelles "TLL" führt auf analoge Ergebnisse.

Die Dissipationsleistung ist nach Gleichung (6.30) für ein Element

$$
\dot{A}_{i}^{e}=\left\{\dot{\alpha}^{q}\right\}^{\top} F^{e}\left\{c^{q}\right\}=\left\{\dot{\beta}^{q}\right\}^{\top}\left\{c^{q}\right\}
$$


Die Summation über alle Elemente führt auf

$$
\begin{aligned}
\dot{A}_{i} & =\sum_{e}\{\dot{\beta}\}^{\top}\left[L_{q}^{e}\right]\left[L_{q}^{e}\right]^{\top}\{c\} \\
& =\{\dot{\beta}\}^{\top}\{c\}
\end{aligned}
$$

Die Leistungen der Knotenlasten nach Gleichung (6.44) und die Dissipationsleistung nach Gleichung (6.50) müssen sich aufheben.

$$
\lambda\{p\}^{\top}\{\dot{w}\}=\{\dot{\beta}\}^{\top}\{c\}
$$

Nach dem Tableau 6.3 muss im dualen Problem

$$
\lambda=\{c\}^{\top}\{\dot{\beta}\}
$$

sein und folglich

$$
-1+\{p\}^{\top}\{\dot{w}\}=0
$$

Das Geschwindigkeitsfeld im Kollapszustand ist also so normiert, dass die Gleichung (6.53) erfüllt ist.

Wenn die Gleichungen (6.52), (6.22) und (6.53) zusammengefasst werden, erhält man das lineare Programm für den kinematischen Grenzwertsatz

$$
\begin{array}{rlr}
\lambda & =\{c\}^{\top}\{\dot{\beta}\} \rightarrow \text { Minimum } \\
\{0\} & =-[G]^{\top}\{\dot{w}\}-[U]^{\top}\{\dot{\beta}\} \\
0 & =-1+\{D\}^{\top}\{\dot{w}\}
\end{array}
$$

Das lineare Programm ist für das Modell "TLL" im Tableau 6.4 dargestellt. Nach dem Hauptsatz der linearen Programmierung [6] ist das nach Gleichung (E.1) berechnete $\lambda$ gleich dem nach Gleichung (6.2) berechneten $\lambda$. 


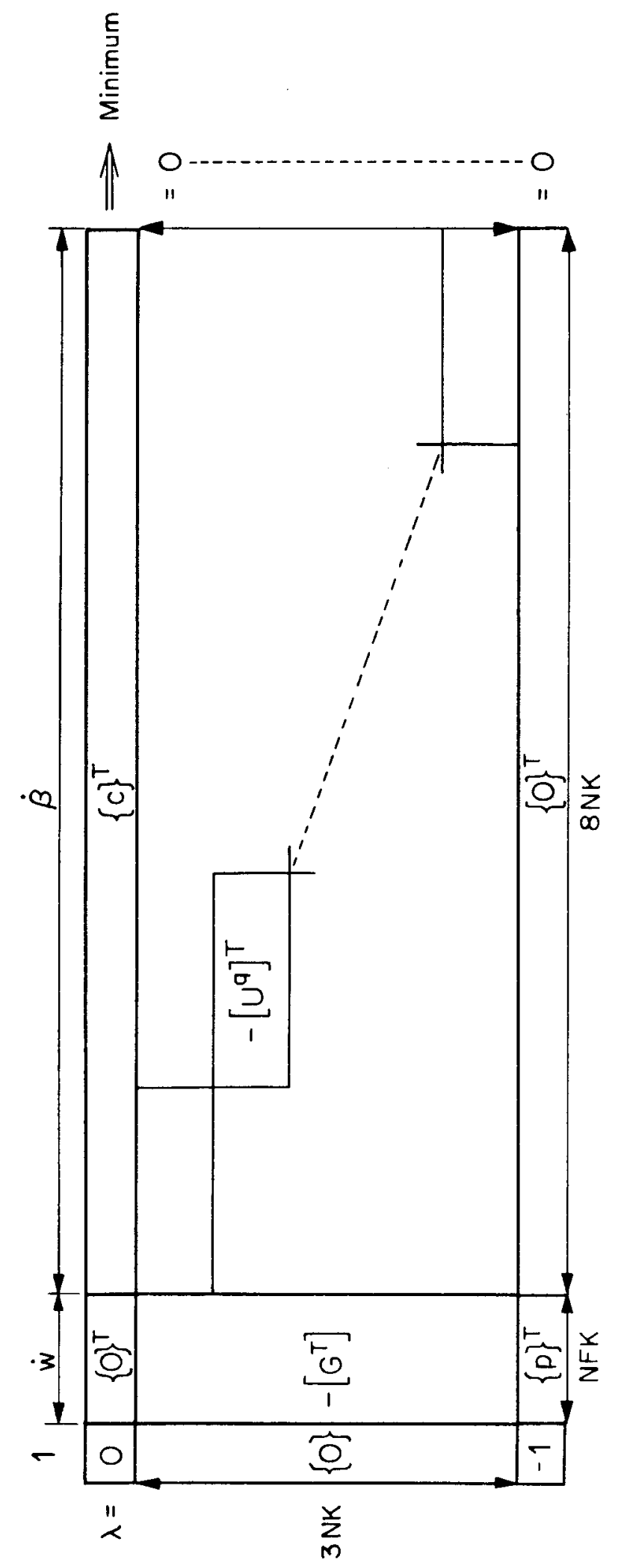

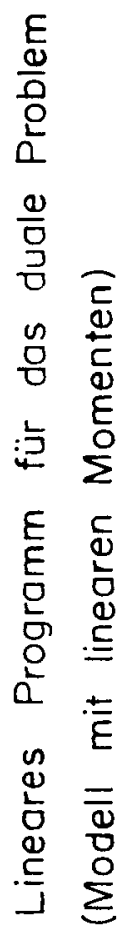

.

$\stackrel{\nabla}{\circ}$

뭉 


\section{OPTIMIERUNGSVERFAHREN}

\subsection{Aufgabe und Lösungsablauf}

Zuerst soll das primale Problem (Tableau 6.1 resp. Tableau 6.2) gelöst werden. Im Abschnitt 7.5 wird dann gezeigt, dass die Lösung des dualen Problems derjenigen des primalen problems vollständig entspricht.

Das primale Problem

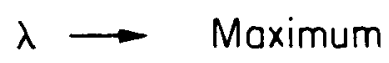

$\begin{array}{rlrl}\text { bezüglich } & \{y\} & =[U]\{m\}+\{c\} \\ \text { wobei } & \{y\} \geqq\{0\} \\ \text { und } & \{0\}=[G]\{m\}-\lambda\{p\}\end{array}$

ist ein lineares Optimierungsproblem für positive (genau genommen: nichtnegative) und freie Variable mit gemischten Restriktionen [6]. Ferner wurde im Abschnitt 2.2 festgelegt, dass die Koeffizienten des Konstantenvektors \{c\} positiv sein sollen. Damit ist die Basislösung $\{y\}=\{c\}$ zum Problem (7.1) zulässig. Sie ist aber nicht optimal, weil das zugehörige $\lambda$ null ist. Die optimale, zulässige Lösung des Problems wird in folgenden Stufen erreicht

- Mittels der Gleichgewichtsgleichungen können einige freie Variable $\left\{m_{1}\right\}$ durch die restlichen freien Variablen $\left\{m_{2}\right\}$ ausgedrückt werden.

- Auch die übrigen freien Variablen $\left\{m_{2}\right\}$ werden eliminiert, indem sie als Funktion eines Satzes von positiven Variablen $\left\{y_{a}\right\}$ ausgedrückt werden.

- Die positiven Variablen werden so lange nach der Optimierungsregel ausgetauscht, bis die optimale Lösung erreicht ist.

- Die Werte aller Variablen für die primale und die duale optimale Lösung werden berechnet. 


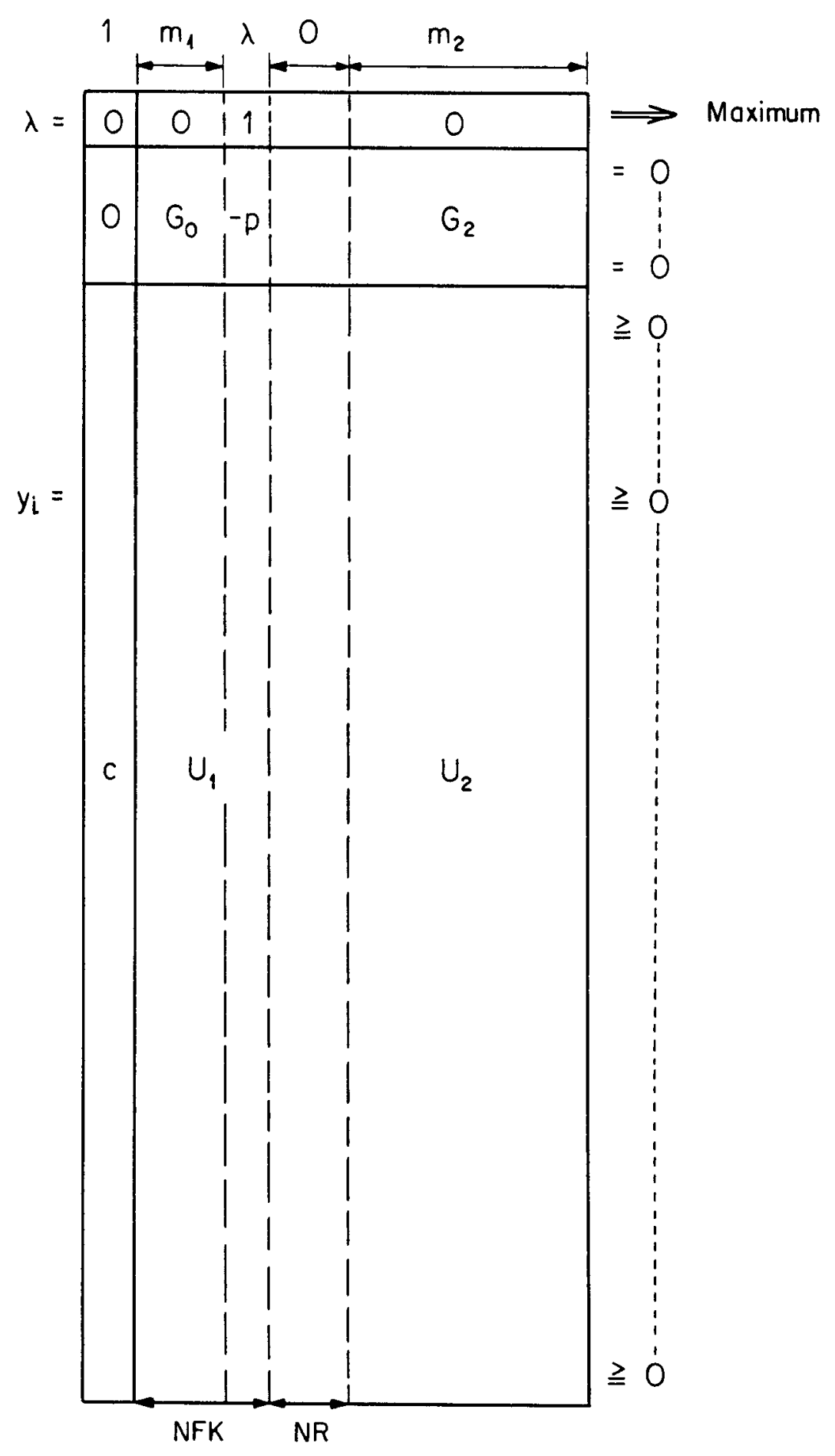

Tableau 7.1: Umordnung der freien Variablen 


\subsection{Elimination der Gleichungen}

Die Gleichgewichtsbedingungen werden dazu benützt, NFK-1 freie Variable $\left\{m_{1}\right\}$ und den Traglastfaktor $\lambda$ durch die übrigen freien Variablen $\left\{m_{2}\right\}$ auszudrücken. Mit NFK wird wieder die Anzahl der freien Knoten bezeichnet. Die Anzahl der freien Variablen, die infolge Randbedingungen verschwinden, sei NR. Die zugehörigen Kolonnen haben auf die folgende Berechnung keinen Einfluss und können weggelassen werden. Die Gleichgewichtsbedingungen können folgendermassen formuliert werden (Tableau 7.1):

$$
\begin{aligned}
& {\left[\begin{array}{c:c:c}
G_{0} & -p & G_{2}
\end{array}\right]\left\{\begin{array}{c}
m_{1} \\
\hdashline \lambda \\
\hdashline m_{2}
\end{array}\right]=\{0\}} \\
& \left\{\begin{array}{c}
m_{1} \\
\hdashline \lambda
\end{array}\right\}=-\left[G_{0}:-p\right]^{-1}\left[G_{2}\right]\left\{m_{2}\right\}=-\left[G_{1}\right]^{-1}\left[G_{2}\right]\left\{m_{2}\right\}
\end{aligned}
$$

Der Vektor $\left\{m_{1}\right\}$ ist so zu wählen, dass $\left[G_{1}\right]$ nichtsingulär wird. Die Ungleichungen können ebenfalls in Funktion der Momente $\left\{m_{2}\right\}$ ausgedrückt werden.

$$
\begin{aligned}
& \{y\}=\left(\left[U_{2}\right]-\left[U_{1}\right]\left[G_{1}\right]^{-1}\left[G_{2}\right]\right)\left\{m_{2}\right\}+\{c\} \geqq\{0\} \\
& \{y\}=\left[\bar{U}_{2}\right]\left\{m_{2}\right\}+\{c\}
\end{aligned}
$$

Die Beziehungen (7.3) und (7.4) können als Resultat von NFK Austauschschritten betrachtet werden, wobei die $\left\{m_{1}\right\}$ und $\lambda$ gegen die Nullen getauscht werden. Pivotsuche in der Zeile ist notwendig, damit $\left[G_{1}\right]$ nicht singulär wird. Der Traglastfaktor wird wie eine freie Variable ausgetauscht, und die entsprechende Zeile wird zur Zielfunktion. Die Situation am Ende der Elimination der Gleichungen ist im Tableau 7.2 dargestellt. Die oberste Zeile aus dem Tableau 7.1 würde dasselbe aussagen wie die unterste Gleichung im Tableau 7.2 und wird im folgenden weggelassen. 


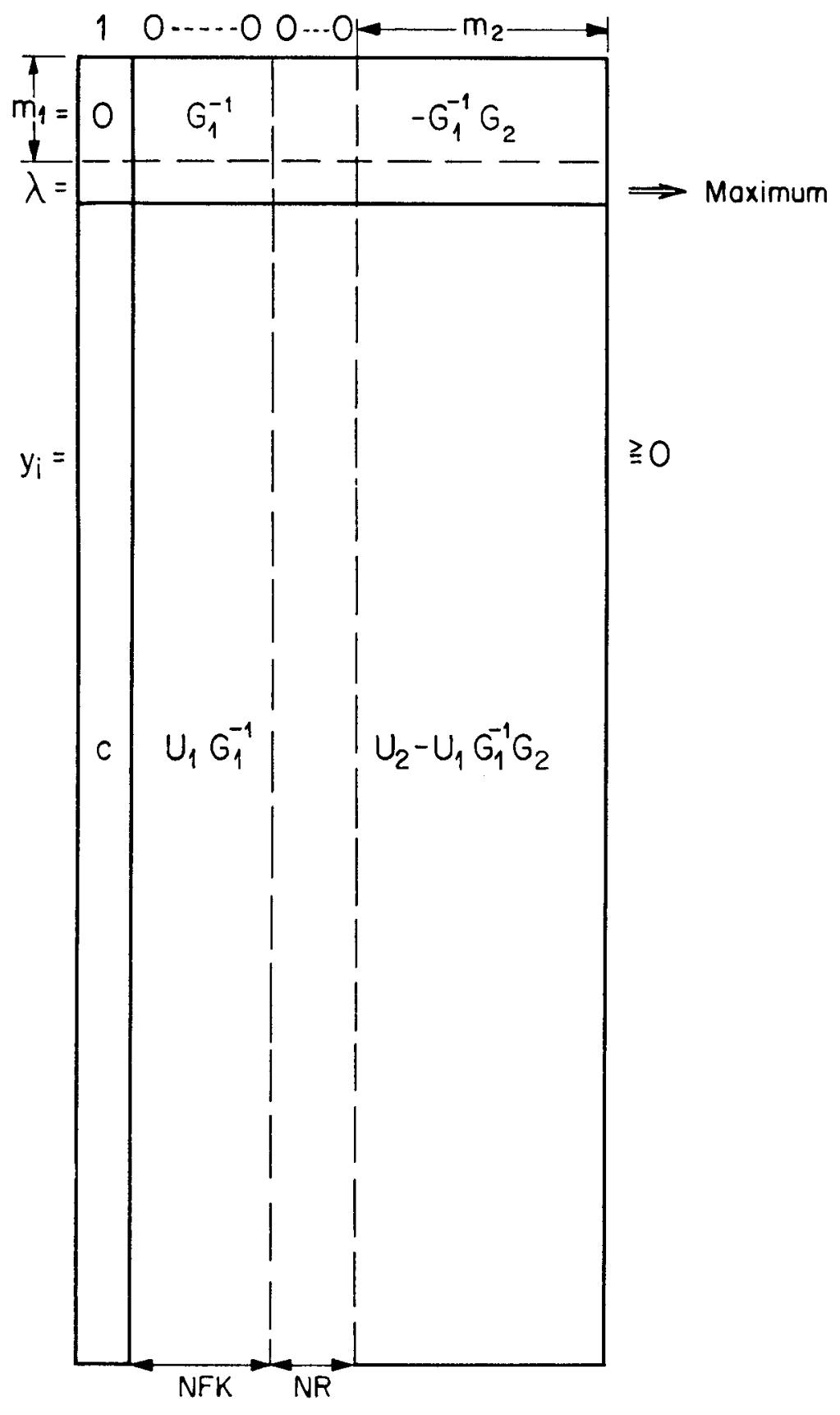

Tableau 7.2: Tableau nach Elimination der Gleichungen 


\subsection{Elimination der übrigen freien Variablen}

Bisher veränderte sich der Konstantenvektor $\{c\}$ nicht. Das lineare Programm liesse sich erweitern, indem man einen konstanten Lastanteil einführen würde.

$$
\{\bar{p}\}=\left\{p_{0}\right\}+\lambda\{p\}
$$

Dann würde sich $\{c\}$ während der Elimination der Gleichungen ändern und es müsste z.B. für den r-ten Austauschschritt verlangt werden, dass

$$
c_{j}^{(r+1)}=c_{j}^{(r)}+\frac{P_{o i} u_{j k}}{g_{i k}} \geqq 0
$$

d.h., es wäre eine zulässige Basislösung $\{y\}\left(\left\{m_{2}\right\}=0\right) \geqq\{0\}$ zu suchen. Die Sicherheitsfaktoren in den Normen sind jedoch so definiert, dass keine konstanten Lastanteile vorkommen.

Bei der Elimination der restlichen freien Variablen verändert sich $\{c\}$. Der Pivot wird aber so gewählt, dass die Basislösung zulässig bleibt. Als weiteres Kriterium wird verwendet, dass die Konstante der Zielfunktion nicht abnehmen soll. Für die Wahl der Pivotkolonne gibt es keine bestimmte Bedingung (z.B. Koeffizient der Zielfunktion möglichst gross), weil die ausgetauschte Nichtbasisvariable auch negativ sein kann.

Wenn mit $\left\{y_{a}\right\}$ die ausgetauschten positiven Variablen bezeichnet werden, folgt aus der Gleichung (7.4)

$$
\left\{m_{2}\right\}=[B]^{-1}\left\{y_{0}\right\}-[B]^{-1}\left\{c_{0}\right\}
$$

Die quadratische nichtsinguläre Matrix $[B]^{-1}=\left[\bar{U}_{2 a}\right]^{-1}$ wird als inverse Basismatrix bezeichnet. Aus den Gleichungen (7.4) und (7.5) folgt ferner die Beziehung zwischen den nicht ausgetauschten positiven Variablen $\left\{y_{b}\right\}$ und den ausgetauschten positiven Variablen $\left\{y_{a}\right\}$ :

$$
\left\{y_{b}\right\}=\left[\bar{U}_{2 b}\right]\left([B]^{-1}\left\{y_{a}\right\}-[B]^{-1}\left\{c_{a}\right\}\right)+\left\{c_{b}\right\}
$$




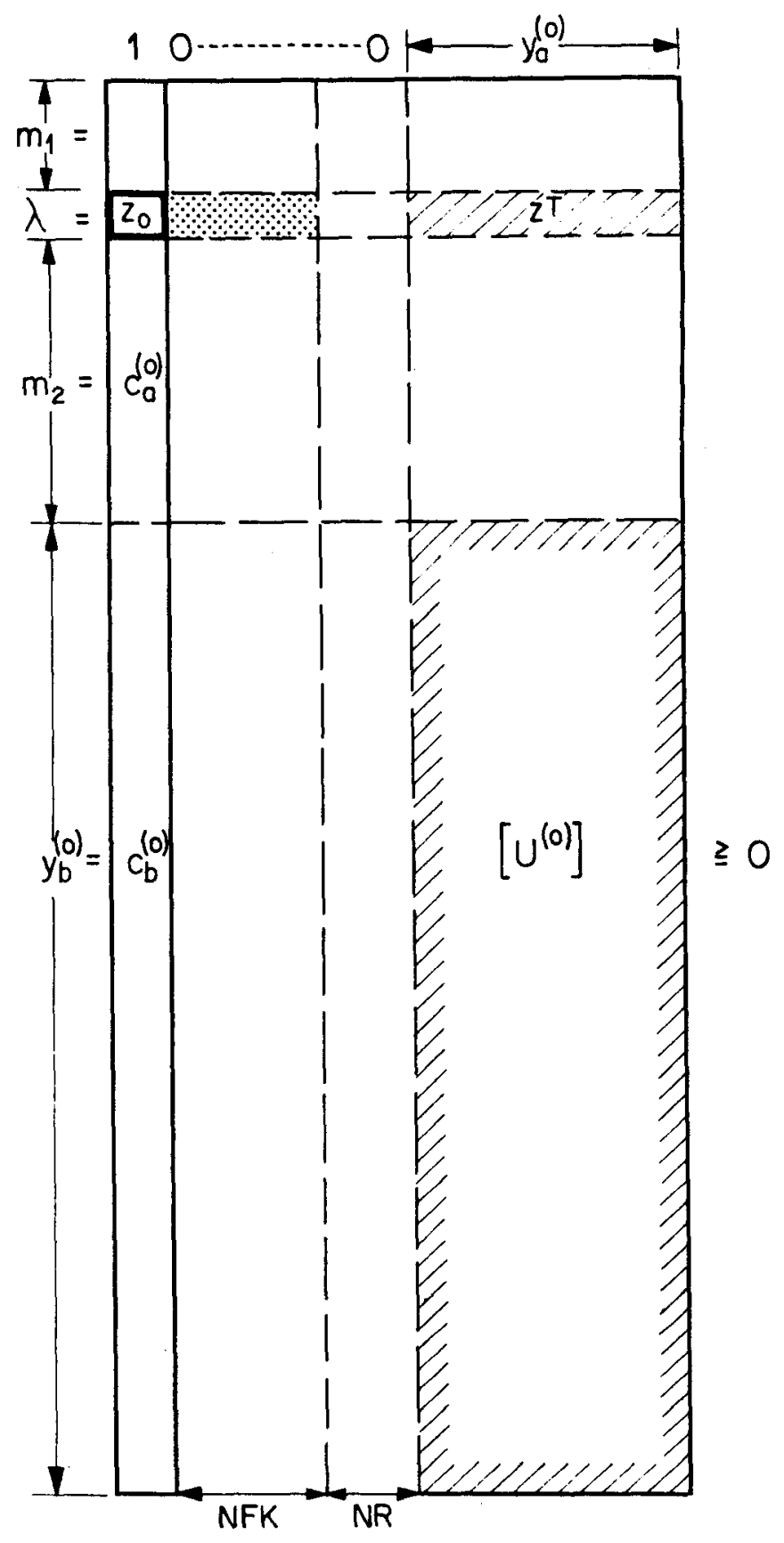

Tableau 7.3: Geordnetes Tableau nach der Elimination der freien Variablen. 
Das Tableau 7.3 zeigt die Ausgangslage für die Optimierung. Alle Basis- und Nichtbasisvariablen sind positive Variable und die Austauschschritte können gemäss den bekannten Kriterien [5] ausgeführt werden. Wenn alle Koeffizienten im schraffierten Teil der Zielfunktion negativ sind, ist der optimale Wert von $\lambda, d . h$. der optimale, zulässige Spannungszustand erreicht, indem $\left\{y_{a}\right\} \equiv 0$ gesetzt werden kann. Die Berechnung der Traglast von starr-plastischen Stahlbetonplatten führt meistens auf vielfach degenerierte systeme.

Ein degeneriertes System liegt vor, wenn sich der Traglastfaktor $\lambda$ bei einem Austauschschritt nicht verändert. Das ist dann der Fall, wenn im Tableau 7.3 entweder der Pivotzeilen-Koeffizient $c_{i}$ der Konstantenkolonne $\left\{c_{b}\right\}$ oder der Pivotkolonnen-Koeffizient $z_{j}$ der Zielfunktion $\{z\}^{\top}$ verschwindet. Bei praktischen Problemen sind meistens mehrere koeffizienten der konstantenkolonne $\{c\}$ im Tableau 7.1 gleich. Die Matrizen $\left[U_{1}\right]$ und $\left[U_{2}\right]$ haben ebenfalls zwei bis vier gleiche, von Null verschiedene Koeffizienten $u_{i j}$ in einer Kolonne. Aus dem Bild 7.1 geht hervor, dass das system degeneriert, wenn $u_{i_{0} j_{0}}=1$ oder $u_{i_{0} j_{0}}=d$ als Pivot gewählt wird.

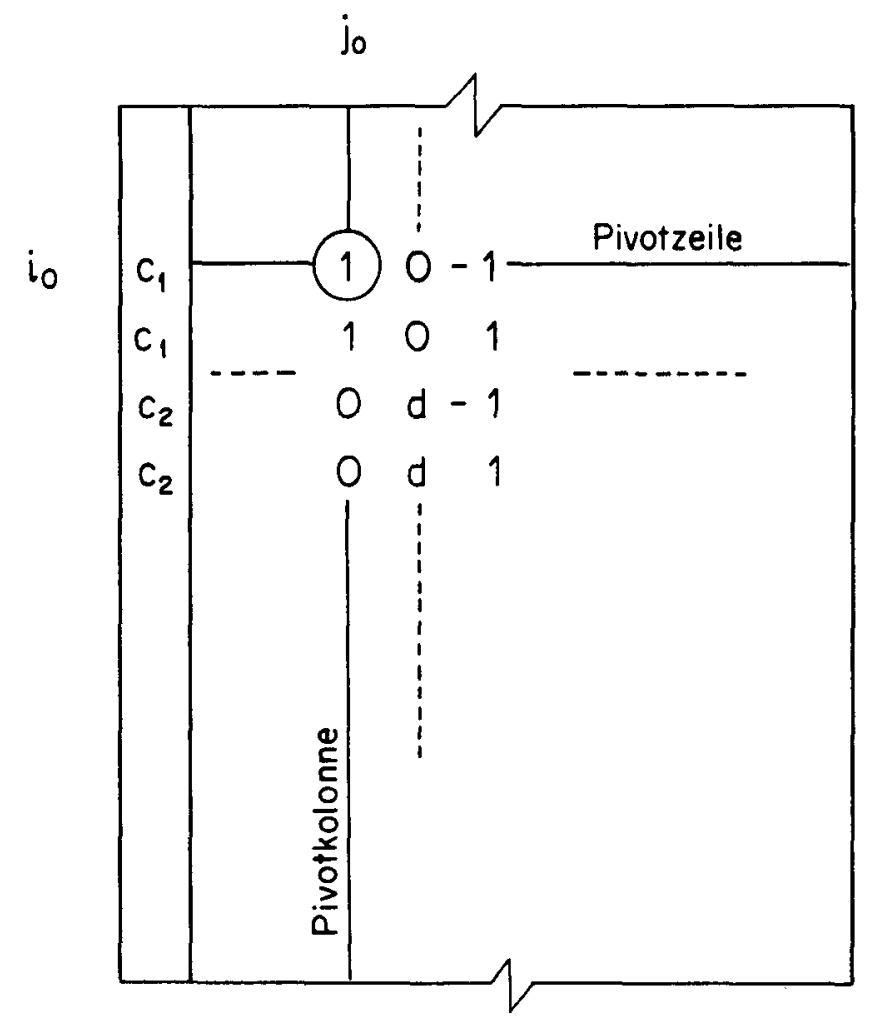

Bild 7.1: Degeneration 
Das bedeutet physikalisch, dass in einem Kontrollpunkt q eine zweite Fliessbedingung erreicht ist (Bild 7.2), ohne dass sich die zugehörige Variable $y_{i}$ ausserhalb der Basis befindet.

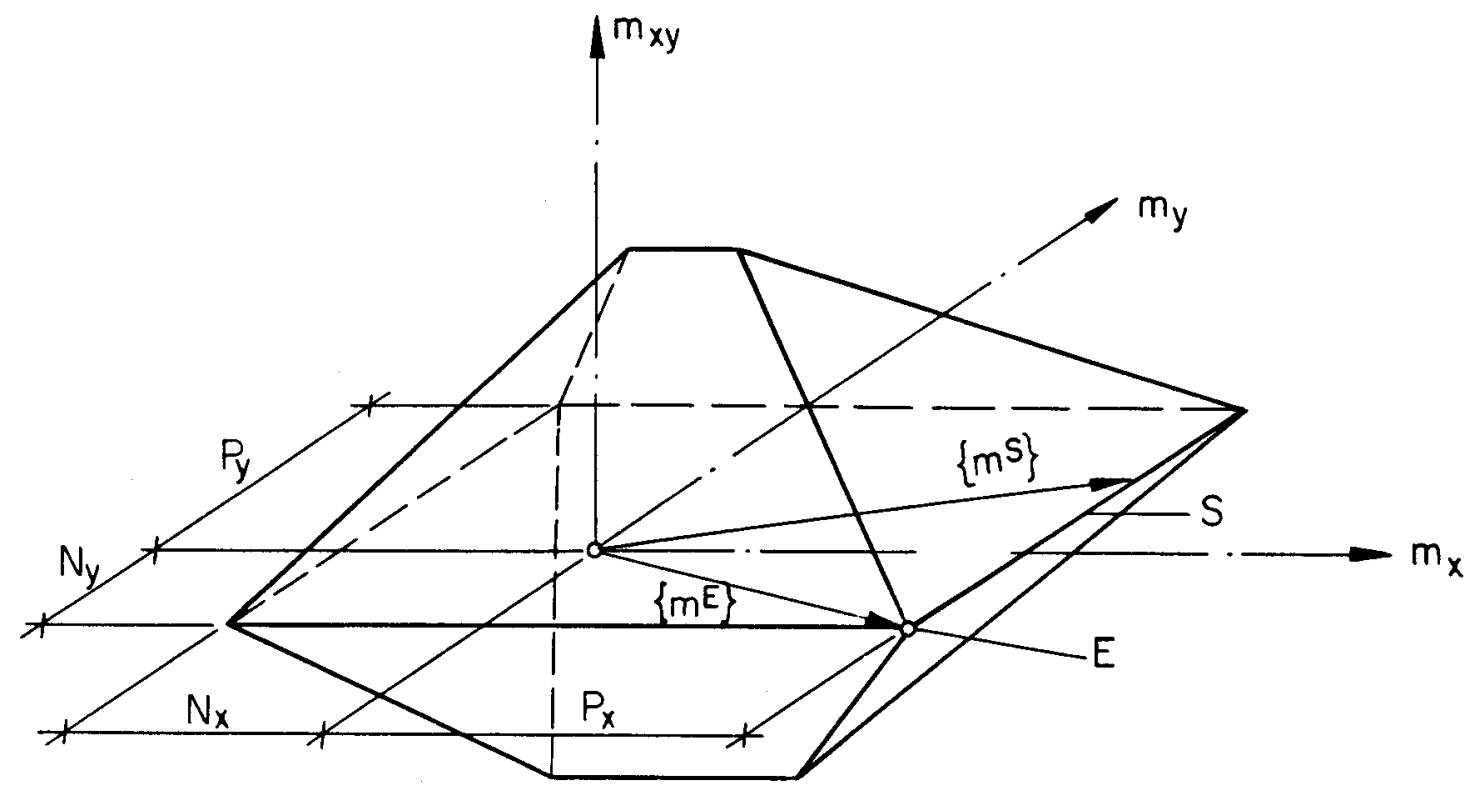

Bild 7.2: Kontrollpunkt mit mehreren erreichten Fliessbedingungen

Die Zeile der Zielfunktion $\{z\}^{\top}$ hat etwa 2 NK Koeffizienten. Wenn alle verschieden von Null wären, müssten für die Basislösung $\left\{y_{a}\right\}=\{0\}$ zusätzlich $z u$ den wegen $c_{i_{0}}=0$ erreichten Fliessbedingungen noch etwa 2 NK Fliessbedingung erreicht sein. Oft sind aber ganze Bereiche der Platte beim Erreichen der Traglast nicht plastifiziert, so dass weniger als 2 NK Fliessbedingungen erreicht sind und somit koeffizienten der Zielfunktion verschwinden müssen.

Die strenge Bedingung $\lambda^{(r+1)}>\lambda^{(r)}$ ist also meistens nicht erfüllt, sondern nur $\lambda^{(r+1)} \geqq \lambda^{(r)}$. Mit $(r)$ wird der $r$-te Austauschschritt bezeichnet. Trotzdem sich theoretische mathematische Beispiele konstruieren lassen, die auf zyklisches Verhalten führen, wurde das Optimum bei allen berechneten Beispielen erreicht, was aus physikalischen Gründen zu erwarten war.

Das Simplexverfahren wird numerisch instabil, wenn ein plastischer Widerstand $c_{i} \cong 0$ ist. Das Vorzeichen des Quotienten $c_{i} / u_{i_{0}} j_{0}$ ändert, wenn $c_{i}=+\varepsilon$ in $c_{i}=-\varepsilon$ übergeht. Infolge Rundungsfehlern und Degeneration kann $c_{i}=-\varepsilon$ werden, wenn $c_{i} \cong 0$. Deshalb muss für die numerische Berechnung zur Bedingung

$$
\frac{c_{i_{0}}}{u_{i_{0} j_{0}}} \geqq \frac{c_{i}}{u_{i} j_{0}} \quad\left(u_{i_{0} j_{0}}<0, c_{i_{0}}>0\right)
$$


für die Wahl der auszutauschenden Basisvariablen noch die Bedingung

$$
\left|u_{\text {iojo }}\right| \geqq \epsilon_{0} \quad\left(z . B . \quad \epsilon_{0}=10^{-7}\right)
$$

für den Pivotkoeffizient hinzugefügt werden. Die Grösse von $\varepsilon_{0}$ kann als absoluter Wert angegeben werden, weil die Koeffizienten der Ungleichungsmatrix dimensionslose Grössen sind. Die Wahl von $\varepsilon_{0}$ kann nur auf empirischem Weg erfolgen. Es gibt zwar Grenzwerte und Erfahrungswerte für die Fortpflanzung von Rundungsfehlern für spezielle Fälle (z.B. Lösung von Gleichungssystemen). Im vorliegenden Fall ist jedoch der Austauschschritt eine kompliziertere Operation und vor allem ist die Anzahl der notwendigen Optimierungsschritte selbst von den Rundungsfehlern und damit von der Numerierung der Elemente abhängig. Wenn die Genauigkeit nicht mehr reicht, kann ein beliebiges Resultat für $\lambda$ erscheinen. Es ist deshalb wesentlich, dass der kleinste verwendete Pivot kontrolliert wird.

Als Abbruchkriterium des Optimierungsalgorithmus ist eine bezogene Grösse zu wählen. Für Quadrat- und Kreisplatten hat das Optimum die Form

$$
\lambda p=c_{\lambda} \frac{m_{p}}{l^{2}}, \lambda=c_{\lambda} \cdot \frac{m_{p}}{p l^{2}}
$$

Wenn die Zielfunktion noch kleine positive Koeffizienten $\varepsilon_{z i}$ hat, ist

$$
\lambda_{\text {max }}=c_{\lambda} \frac{m_{p}}{p l^{2}}+\left\{\epsilon_{z}\right\}^{\top}\left\{m_{p}\right\}
$$

Als Abbruchkriterium wurde deshalb für die Computerprogramme

$$
\left.\epsilon_{z i} \leqq \epsilon_{\lambda} \cdot \frac{1}{p^{2}}=\frac{\epsilon_{\lambda}}{P_{\text {tot }}} \quad \text { (z.B. } \epsilon_{\lambda}=10^{-3}\right)
$$

gewählt, wobei $P_{\text {tot }}$ die Summe der Komponentenbeträge des Belastungsvektors \{p\} ist.

Ist das Optimum ermittelt, so können die Traglast und der zugehörige Spannungszustand leicht gefunden werden.

Der Traglastfaktor $\lambda$, d.h. das Verhältnis der Traglast zur Last, die für die Berechnung des Vektors $\{p\}$ angenommen wurde, ist der koeffizient $z_{0}$ in der Konstantenkolonne der Zielfunktion. 
Die Momente bilden einen zulässigen Spannungszustand. Sie sind in den Vektoren $\left\{m_{1}\right\}$ und $\left\{m_{2}\right\}$ enthalten. Nach dem Tableau 7.3 ist

$$
\left\{m_{2}\right\}^{(o p t)}=\left\{c_{a}\right\}^{(o p t)}
$$

weil $\left\{y_{a}\right\}^{\text {(opt })}=\{0\}$ gesetzt werden darf. Der Vektor $\left\{m_{1}\right\}$ kann mittels der Gleichung (7.3) berechnet werden. Aus den Momenten können mit den Gleichungen (4.4) resp. (5.4) die zugehörigen Reaktionen berechnet werden.

Der mit dem linearen Programm berechnete Spannungszustand entspricht jedoch im allgemeinen dem wirklichen Spannungszustand beim kollaps nicht, weil mehrere optimale zulässige Spannungszustände möglich sind. Im Bild 7.3 ist als einfaches Beispiel ein Durchlaufträger dargestellt.
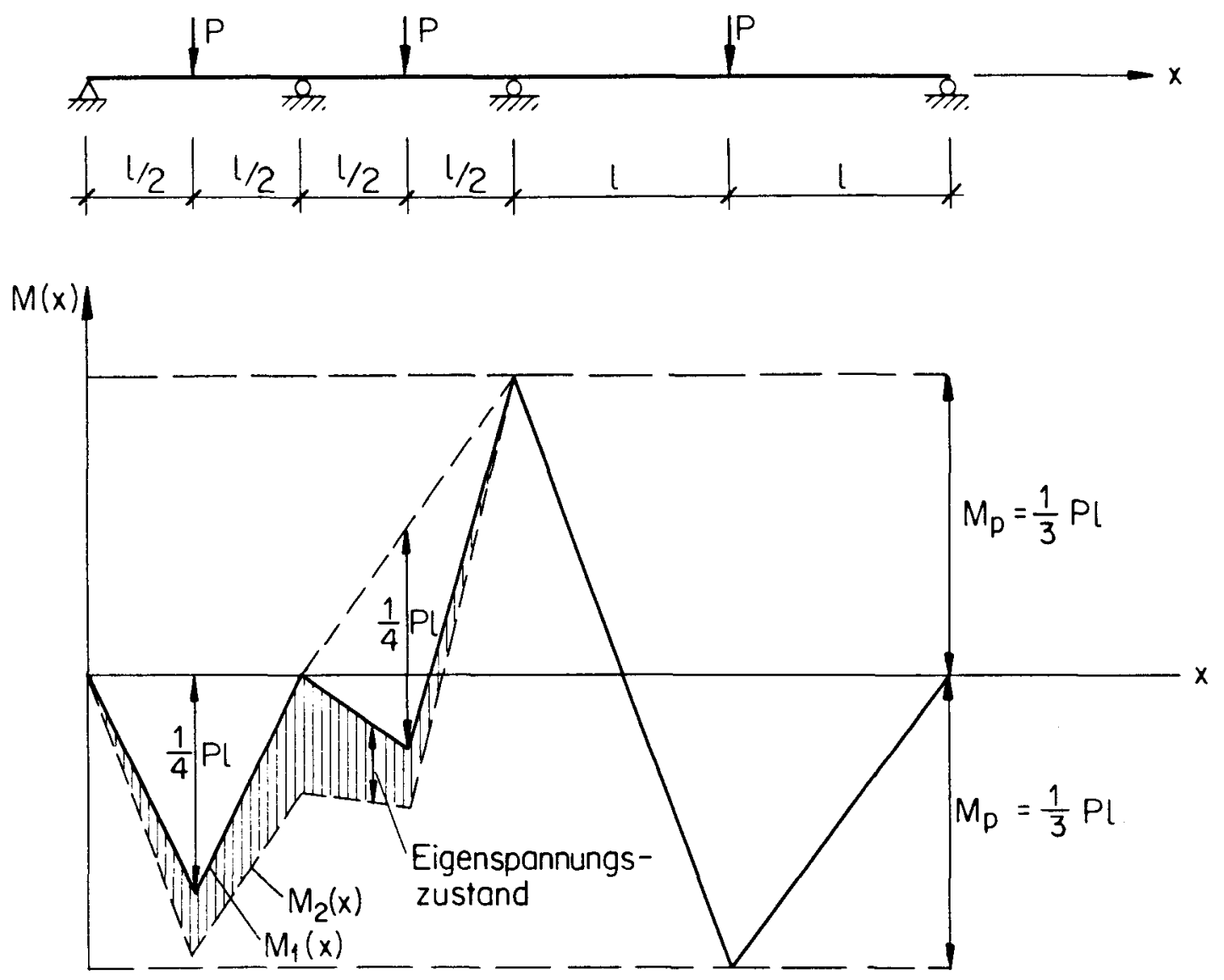

Bild 7.3: Zwei optimole zulässige Gleichgewichtszustönde

Der wirkliche Spannungszustand bei Erreichen der Traglast ist von der Belastungsgeschichte abhängig und kann mit der hier verwendeten Methode nicht ermittelt werden. Deshalb entsprechen die berechneten Momente und Reaktionen in der Regel nur einem möglichen, nicht aber dem wirklichen Zustand. 


\subsection{Duales Problem}

Die Lösung des dualen Problems entspricht bei jedem Schritt genau derjenigen des primalen Problems.

Die Elimination der Gleichungen im primalen Problem (Tableau 7.2) entspricht der Berechnung von $\{\dot{w}\}$ im dualen Problem (Tableau 6.4).

$$
\begin{aligned}
& \left\{\begin{array}{c}
0 \\
-1 \\
-1
\end{array}\right\}=\left[G_{1}^{\top}\right]\{\dot{w}\}+\left[U_{1}^{\top}\right]\{\dot{\beta}\} \\
& \{\dot{w}\}=-\left[G_{1}^{T}\right]^{-1}\left[U_{1}^{\top}\right]\{\dot{\beta}\}-\left[G_{1}^{\top}\right]^{-1}\left\{\begin{array}{c}
0 \\
-1
\end{array}\right\}
\end{aligned}
$$

Den Nullen resp. Momenten im primalen Problem entsprechen $\dot{w}^{i}$ resp. Nullen im dualen Problem. Die Situation nach Berechnung von $\{\dot{w}\}$ ist im Tableau 7.4 dargestellt.

Es stellt sich die Frage, ob die Basislösung $\{\dot{w}\}(\{\dot{\beta}\}=\{0\})$ auch beim dualen Problem zulässig sei. Man stellt fest, dass dies nicht der Fall ist, weil $-\{z\} \neq\{0\}$ ist. Dagegen ist die Basislösung optimal, weil die Lösung $\lambda=0$ zufolge der Bedingungen $\{c\} \geqq\{0\}$ und $\{\dot{\beta}\} \geqq\{0\}$ offensichtlich ein Minimum ist. Man startet also beim dualen Problem mit einer optimalen, nicht zulässigen Basislösung, währenddem man beim primalen Problem mit einer zulässigen, nicht optimalen Basislösung begann.

In der nächsten Phase kann aus den restlichen Gleichungen ein Teil der $\dot{\beta}_{i}$ berechnet werden. Dabei soll aber die Basislösung optimal bleiben, d.h. die Koeffizienten $c_{i}$ dürfen nicht negativ werden. Die gleiche Bedingung gilt auch für die Phase der Optimierung, so dass direkt diese Phase untersucht werden kann. Das primale und das duale Problem sind im Tableau 7.5 wiedergegeben.

Die Vorschrift für die Suche des Pivots im primalen Problem lautet: Suche den grössten Koeffizient $z_{j}$ der Zielfunktion und bestimme den Pivotkoeffizient $u_{i j}$ in der zugehörigen Kolonne so, dass beim Austausch alle Koeffizienten $c_{i}$ der Konstantenkolonne positiv bleiben: $\max \left(c_{i} / u_{i j}\right)$, wobei $u_{i j}<0$. Wenn alle $z_{j}$ nichtpositiv sind, ist das Maximum erreicht. Die Koeffizienten werden immer algebraisch aufgefasst, d.h. a < b bedeutet, dass a auf der Zahlengerade weiter links steht als $b$ : $-\infty<a<b<+\infty$. 


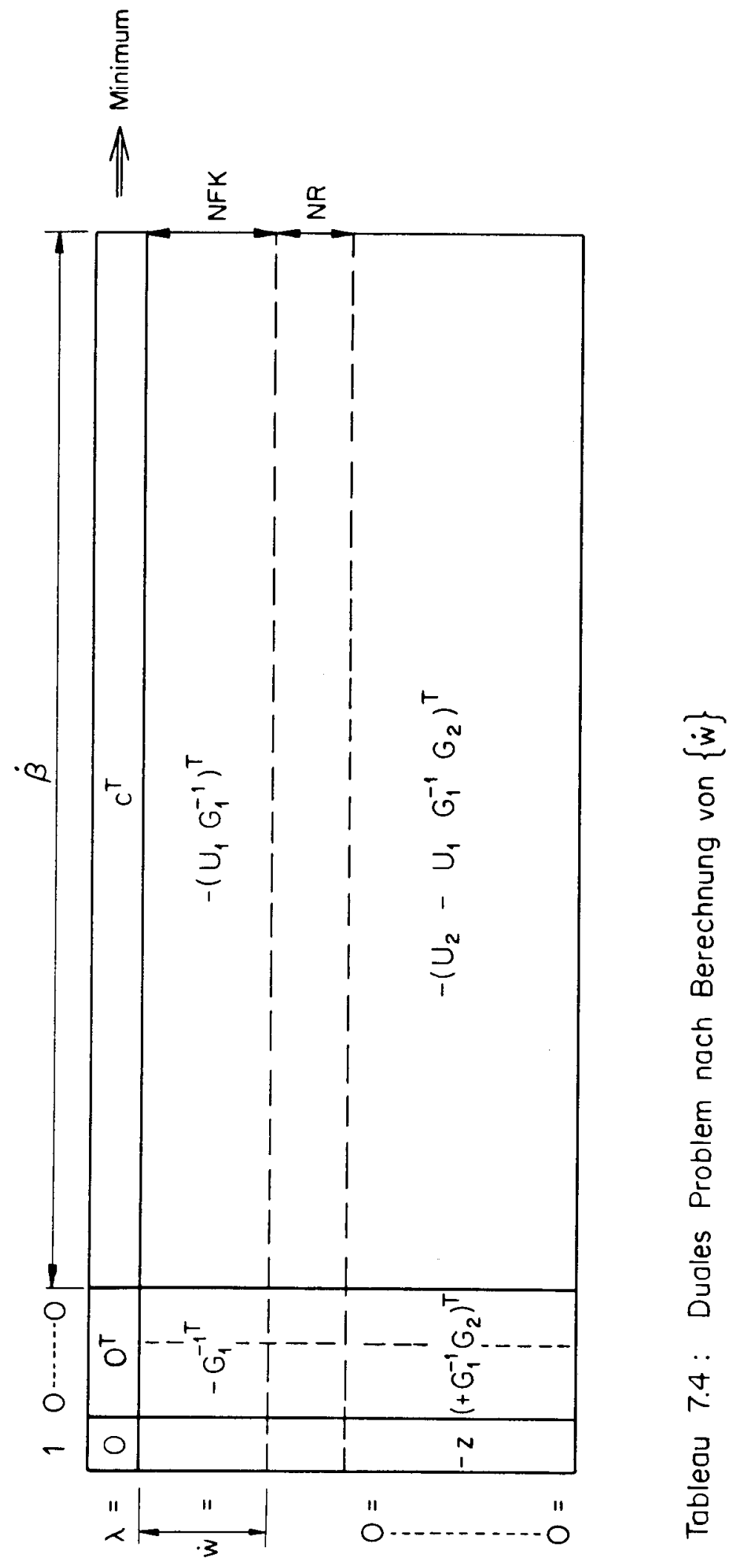




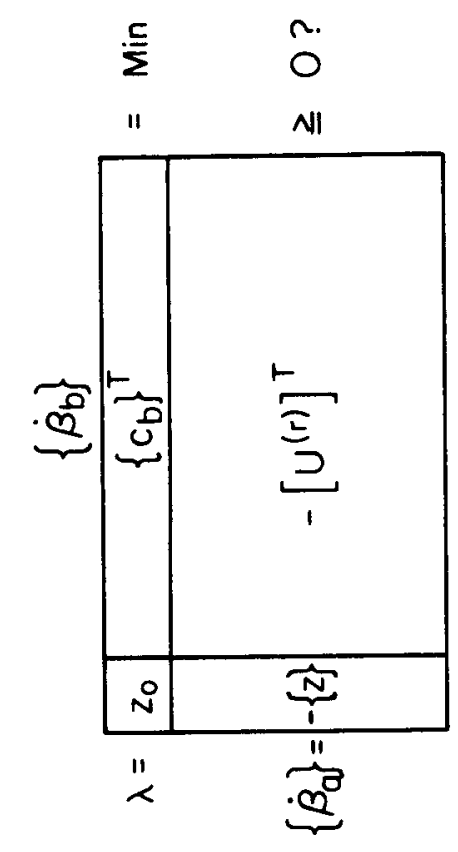

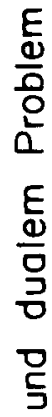

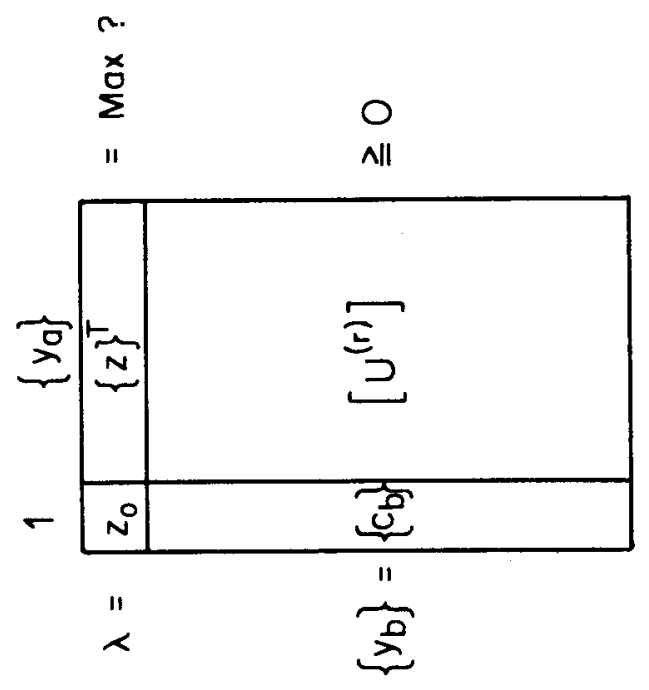

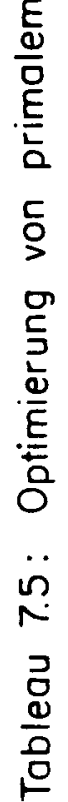


Die Vorschrift für die Suche des Pivots im dualen Problem lautet: Suche den kleinsten Koeffizient $-z_{j}$ der Konstantenkolonne und bestimme den Pivotkoeffizient $u_{j i}$ in der zugehörigen Zeile so, dass beim Austausch alle Koeffizienten $c_{j}$ der Zielfunktion positiv bleiben: min $\left(c_{i} /-u_{j i}\right)$, wobei $-u_{j i}>0$. Wenn alle $-z_{j}$ nichtnegativ sind, ist die Basislösung zulässig.

Man stellt fest, dass sich die Vorschriften vollständig entsprechen. Somit werden bei jedem Austauschschritt die sich entsprechenden Variablen ausgetauscht. Die Tableaux entsprechen sich in jedem Moment des Optimierungsverfahrens. Wenn also z.B. das primale Problem gelöst wurde (Tableau 7.3), können die Werte für die Variablen des dualen Problems direkt aus dem "transponierten Tableau" 7.6 herausgelesen werden. Die Lösung $\left\{\dot{\beta}_{b}\right\}=\{0\}$ und $\left\{\dot{\beta}_{a}\right\}=-\{z\}^{\text {(opt) }}$ ist zulässig und optimal.

Der Vektor $\{\dot{\beta}\}$ ist damit bekannt und der Mechanismus $\{\dot{w}\}$ kann mittels der Gleichung (7.13) berechnet werden.

Im Gegensatz zum Spannungszustand $\{m\}$ ist der Mechanismus $\{\dot{w}\}$ für die optimale Lösung im allgemeinen eindeutig bestimmt. Der folgende Spezialfall ist noch zu erwähnen: Haben zwei Mechanismen dieselbe Traglast, so können lineare Kombinationen der entsprechenden Mechanismen als Ergebnis der Berechnung erscheinen. Wie aus dem Bild 7.4 ersichtlich ist, kann trotz Symmetrie in system und Belastung ein unsymmetrischer Mechanismus resultieren.

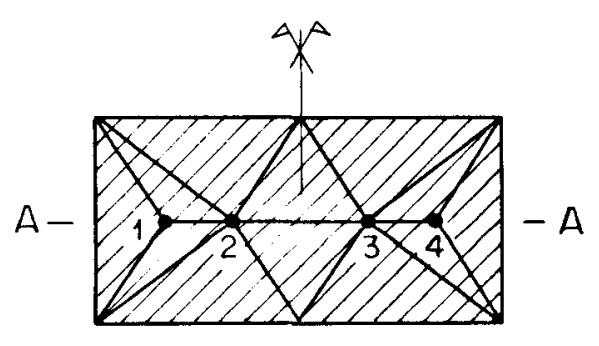

$m_{p} \equiv 1.0$

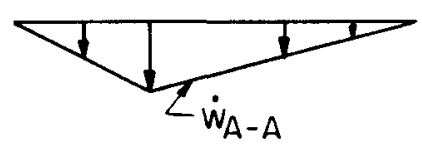

Platte
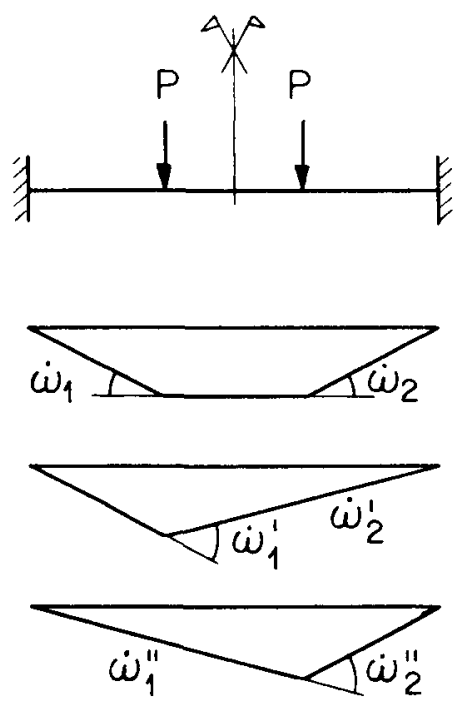

Balken

Bild 7.4: Nicht eindeutig bestimmter Mechanismus 


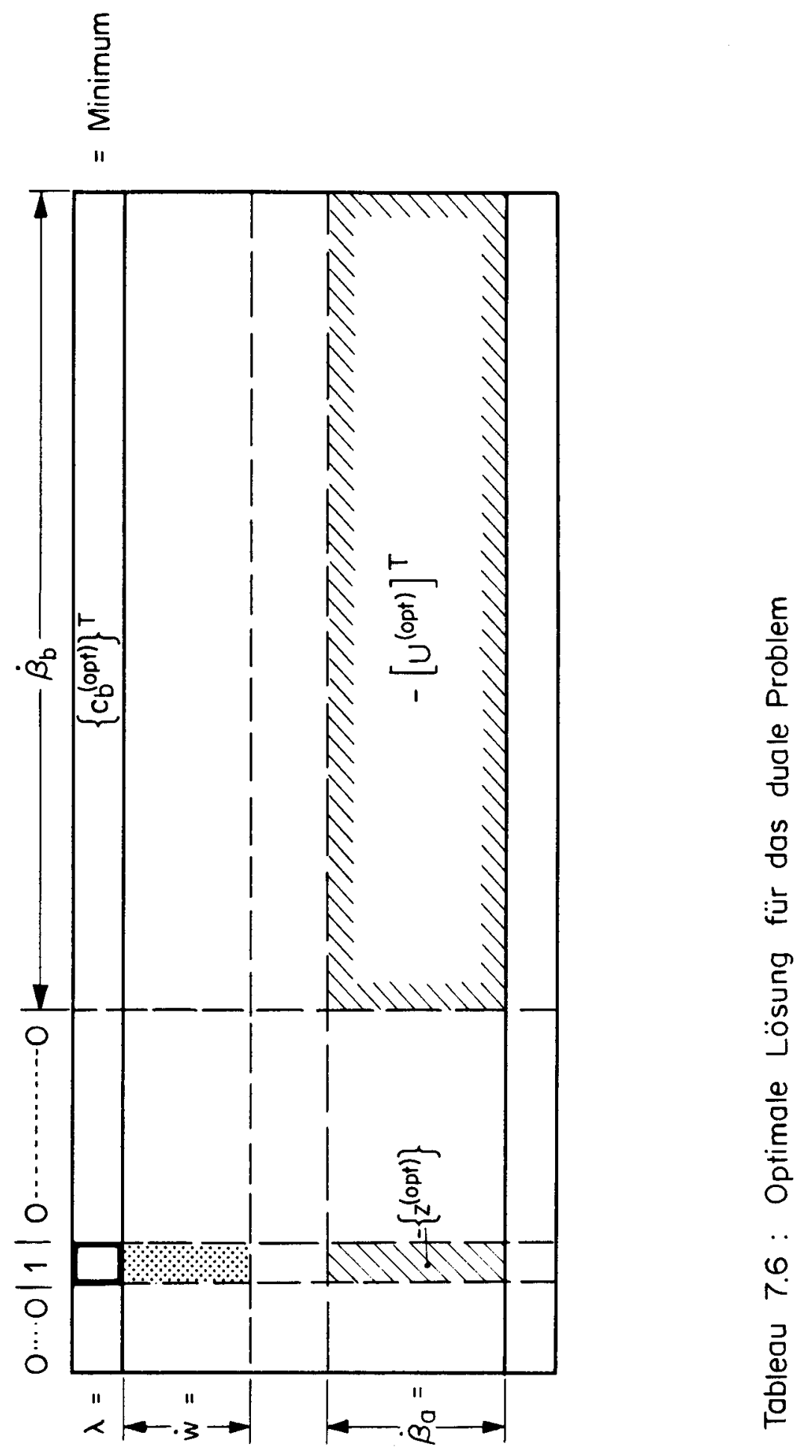


Der resultierende Mechanismus gibt dem Ingenieur ein anschauliches Bild vom Kollapszustand und zeigt auch an, an welchen Orten die grössten Rotationen auftreten müssen. Man erinnert sich auch an die ökonomische Interpretation des dualen Problems zum linearen Produktionsmodell [26]. Wird ein plastischer Widerstand $c_{i}$ um $\Delta c_{i}$ vergrössert, dann vergrössert sich der Traglastfaktor $\lambda$ um den Betrag $\Delta c_{i} \dot{\beta}_{i}$ (Tableau 6.4). Die $\dot{\beta}_{i}$ sind die sogenannten "Schattenpreise", d.h. die Erhöhungen des Traglastfaktors pro hinzugefügte Einheit des betreffenden plastischen Widerstandes. Es lohnt sich also besonders, diejenigen plastischen Widerstände zu vergrössern, die zu einem grossen $\dot{\beta}_{i}$ gehören. Diese Aussage gilt jedoch nur für eine beschränkte Erhöhung $\Delta c_{i}$, weil sich das System durch diese Erhöhung ändern kann (es werden dann andere Plastizitätsbedingungen massgebend).

Die Aehnlichkeit der Resultate mit denjenigen der sogenannten Mechanismusmethode [4] ist auffallend. Der Vergleich ist im Anhang $C$ durchgeführt.

\subsection{Revidiertes Simplexverfahren}

Die ursprüngliche Ungleichungsmatrix [U] hat einen sehr einfachen Aufbau. Es liegt daher nahe, ihre Koeffizienten bei der Berechnung mit dem Computer nicht zu speichern. Das ist möglich, wenn man mit der inversen Basismatrix des revidierten Simplexverfahrens arbeitet.

Man geht davon aus, dass die Gleichungen bereits eliminiert sind (Tableau 7.2). Die Koeffizienten der Ungleichungsmatrix

$$
\left[\bar{U}_{2}\right]=\left[U_{2}\right]-\left[U_{1}\right]\left[G_{1}\right]^{-1}\left[G_{2}\right]
$$

können jederzeit auf einfache Weise ermittelt werden, wenn $\left[G_{1}\right]^{-1}\left[G_{2}\right]$ gespeichert ist.

Cie Austauschoperationen für den Austausch der freien Variablen und diejenigen für den Austausch von positiven Variablen unterscheiden sich nicht. Daher können diese beiden Phasen gemeinsam behandelt werden. In [6] wird das revidierte simplexverfahren für den fall hergeleitet, dass mehr Variatle als Ungleichungen vorhanden sind.

$$
\{y\}=\left[A_{0}\right]\{x\}+\left\{b_{0}\right\}
$$


Dabei sind $y_{i}$ die Basisvariablen (abhängig) und die $x_{j}$ die Nichtbasisvariablen (unabhängig). Beim normalen Simplexverfahren wird bei jedem Austauschschritt die ganze Ungleichungsmatrix [A] verändert.

$$
\{y\}^{(r)}=[A]^{(r)}\{x\}^{(r)}+\{b\}^{(r)}
$$
Die Auswirkungen des r-ten Austauschschrittes $\left(y_{i_{0}}\right.$ gegen $x_{j_{0}}$ ) auf die Matrix
(A] sind im Bild 7.5 dargestellt.

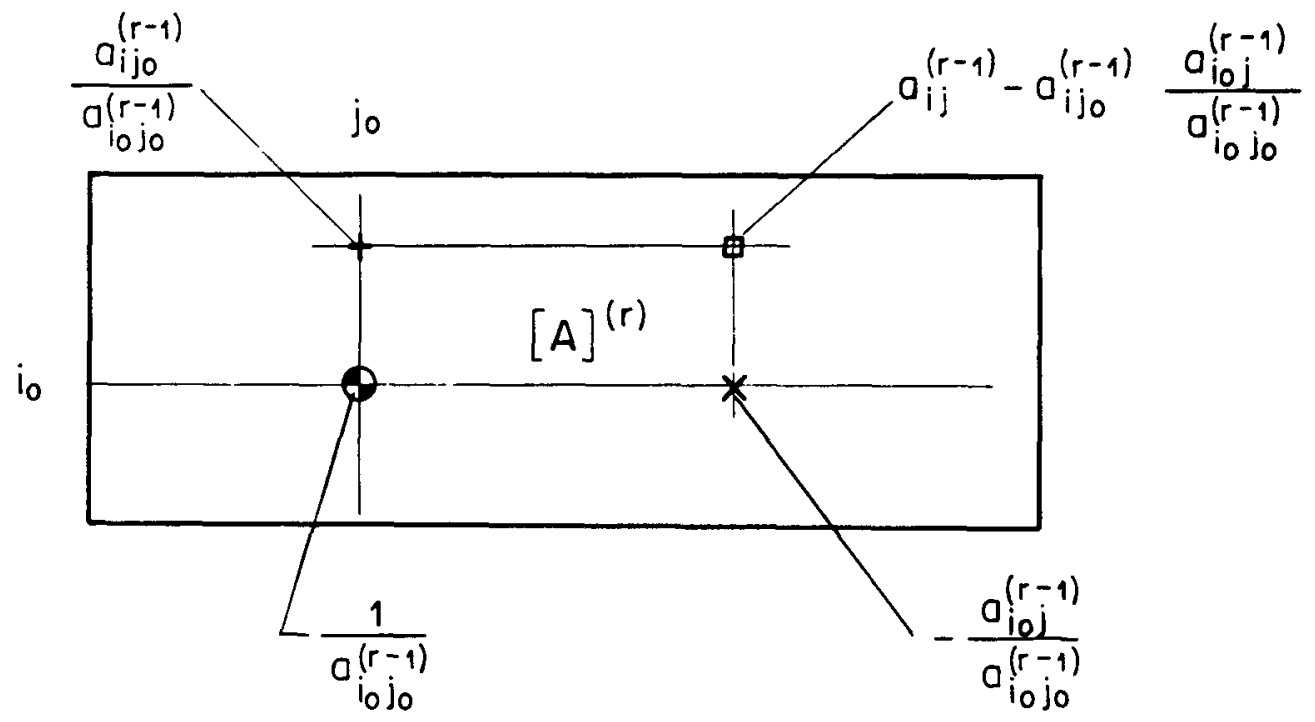

Bild 7.5: Austauschschritt

Man mächte nun eine Transformationsmatrix $\left[T^{T}\right]$ finden, die den r-ten Austauschschritt ausführt.

$$
[A]^{(r)}=\left[T^{T}\right]^{(r)}[A]^{(r-1)} ?
$$

Aus dem Bild 7.5 geht hervor, dass die Transformationsmatrix die folgende Form hat 


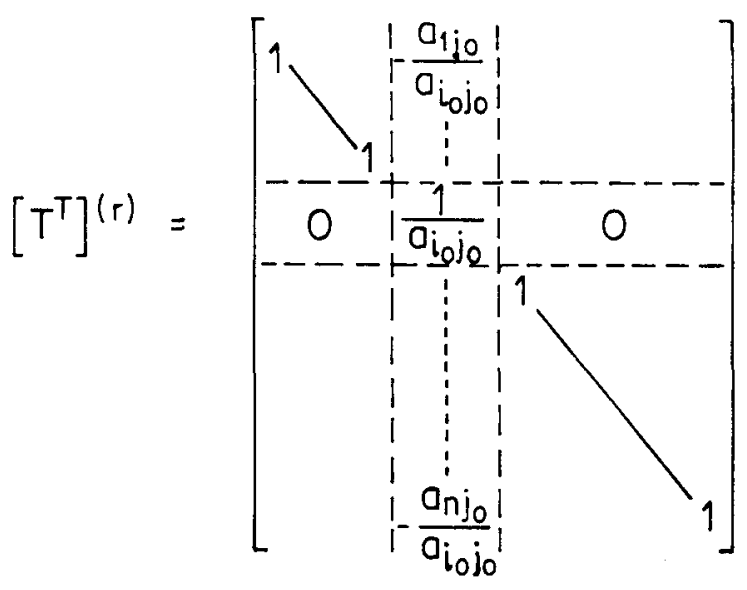

Diese Matrix transformiert alle Koeffizienten mit Ausnahme der Pivotkolonne richtig. Die Matrix $[A]^{(r-1)}$ bleibt praktisch unverändert; einzig die Pivotkolonne ist zu ersetzen durch den Vektor

$$
\left\{\bar{a}_{j 0}\right\}^{(r)}=\left\{\begin{array}{c}
0 \\
\vdots \\
0 \\
1 \\
0 \\
\vdots \\
0
\end{array}\right\}
$$

mit einer Eins in der Zeile $i_{0}$, weil die Variable $x_{j}$ zur abhängigen Variablen geworden ist. Wenn die so modifizierte Matrix $[\AA]]^{(r-1)}$ mit $[\bar{A}](r-1)$ bezeichnet wird, kann die Veränderung der Ungleichungsmatrix durch den r-ten Austauschschritt mittels der Gleichung

$$
[A]^{(r)}=\left[T^{T}\right]^{(r)}[\bar{A}]^{(r-1)}
$$

angegeben werden. Die Veränderungen durch die Austauschschritte (1) bis ( $r$ ) lassen sich folgendermassen in Produktform darstellen:

$$
[A]^{(r)}=\left[T^{T}\right]^{(r)}\left[T^{T}\right]^{(r-1)} \ldots \ldots \ldots \ldots\left[T^{T}\right]^{(1)}\left[A_{0}\right]^{(r)}
$$

Die Matrix $\left[A_{0}\right]^{(r)}$ erhält man aus der ursprünglichen Matrix $\left[A_{0}\right]$, indem man die Kolonnen, die als Pivotkolonnen gewählt wurden, durch die entsprechenden Vektoren (7.19) ersetzt. Das Produkt

$$
\left[B^{-1}\right]^{(r)}=\left[T^{T}\right]^{(r)}\left[T^{T}\right]^{(r-1)} \ldots \ldots \ldots \ldots . . \cdots\left[T^{T}\right]^{(1)}
$$


wird als inverse Basismatrix bezeichnet, weil es die Beziehungen zwischen den ursprünglichen und den aktuellen Basisvariablen darstellt.

Für den supponierten Fall, dass als Pivotkoeffizient der Reihe nach die Diagonalkoeffizienten gewählt werden, ist der Vorgang im Bild 7.6 veranschaulicht.

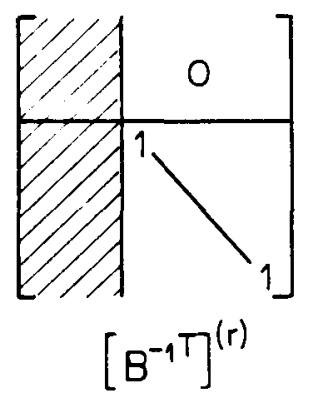

(Gleichung 7.22)

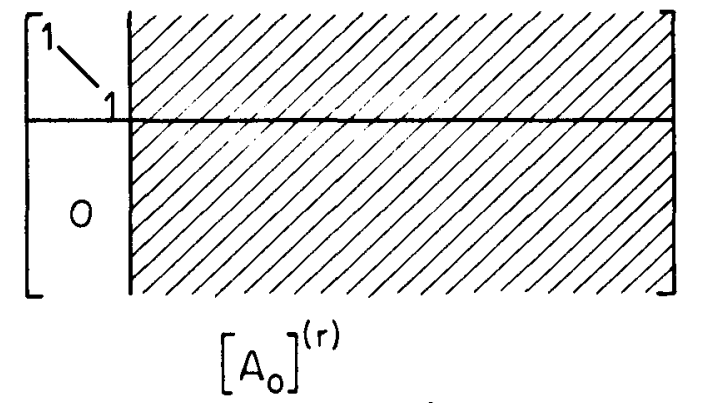

(Gleichung 7.21)

Bild 7.6 : Veranschoulichung des Austausches

Konstantenkolonne und Zielfunktion transformieren sich wie eine gewöhnliche Kolonne resp. Zeile. Es ist z.B.

$$
\{b\}^{(r)}=\left[B^{-1 T}\right]^{(r)}\left\{b_{0}\right\}
$$

Der Austausch der freien Variablen (Abschnitt 7.3) und die Optimierung (Abschnitt 7.4 J können ebenfalls in Produktform angegeben werden, wobei als Transformationsmatrix [T] anstelle von [T $\left.{ }^{\top}\right]$ auftritt. Die Beziehung (7.6) gilt also allgemein und man bekommt

$\left\{y_{b}\right\}^{(r)}=\left[\bar{U}_{2 b o}\right]^{(r)}\left[B^{-1}\right]^{(r)}\left\{y_{a}\right\}^{(r)}+\left\{c_{b o}\right\}^{(r)}-\left[\bar{U}_{2 b o}\right]^{(r)}\left[B^{-1}\right]^{(r)}\left\{c_{a o}\right\}^{(r)}$

In der Matrix $\left[\bar{U}_{2 b o}\right]^{(r)}$ stehen diejenigen Zeilen der ursprünglichen Matrix $\left[\bar{U}_{2}\right]$, die zu Basisvariablen gehören. Beim Konstantenvektor lohnt es sich nicht, die Produktform zu verwenden. Er wird gespeichert und bei jedem Austauschschritt verändert, weil die Pivotkolonne ohnehin bei der Pivotsuche berechnet werden muss. Durch Einsetzen der Gleichung (7.14) ergibt sich 
$\left\{y_{b}\right\}^{(r)}=\left(\left[U_{2 b o}\right]^{(r)}-\left[U_{1 b o}\right]^{(r)}\left[G_{1}^{-1}\right]\left[G_{2}\right]\right)\left[B^{-1}\right]^{(r)}\left\{y_{a}\right\}^{(r)}+\left\{c_{b}\right\}^{(r)}$

Es werden nur die Matrizen $\left[G_{1}^{-1}\right]\left[G_{2}\right]$ und $\left[B^{-1}\right](r)$, sowie die Konstantenkolonne $\{c\}^{(r)}$ und die Zielfunktion $\left\{z^{\top}\right\}^{(r)}$ gespeichert. Anstelle von $\left[G_{1}^{-1}\right]\left[G_{2}\right]$ kann gerade das Produkt $\left[G_{1}^{-1}\right]\left[G_{2}\right]\left[B^{-1}\right](r)$ gespeichert werden, was bedeutet, dass man die Austauschoperationen auch auf $\left[G_{1}^{-1}\right]\left[G_{2}\right]$ anwendet, d.h. die Gleichgewichtsbedingungen in Funktion der aktuellen Nichtbasisvariablen ausdrückt.

$\left\{y_{b}\right\}^{(r)}=\left(\left[U_{2 b o}\right]^{(r)}\left[B^{-1}\right]^{(r)}-\left[U_{1 b o}\right]^{(r)}\left(\left[G_{1}^{-1}\right]\left[G_{2}\right]\right)^{(r)}\right)\left\{y_{a}\right\}^{(r)}+\left\{c_{b}\right\}^{(r)}$

Ein Austauschschritt bedeutet, dass Gleichgewichts- und Plastizitätsbedingungen durch einen neuen Satz von Variablen ausgedrückt werden. Der neue Satz von Variablen unterscheidet sich vom alten dadurch, dass eine der Nichtbasisvariablen durch eine Basisvariable ersetzt wird. Ein Austauschschritt erfordert folgende Operationen:

- die Variablen, welche ausgetauscht werden sollen, werden bestimmt

- die inverse Basismatrix $\left[B^{-1}\right]$ und die Matrix $\left[G_{1}^{-1}\right]\left[G_{2}\right]$ werden mit der Transformationsmatrix [T] nachmultipliziert

- die Matrizen $\left[\bar{U}_{1}\right]$ und $\left[\bar{U}_{2}\right]$ werden gemäss der Gleichung (7.19) modifiziert

- Zielfunktion und Konstantenkolonne werden mittels Pivotzeile und -kolonne transformiert.

Das Tableau 7.7 gibt eine Uebersicht über die verwendeten Matrizen und Vektoren. Gespeicherte Grössen sind schraffiert dargestellt. 


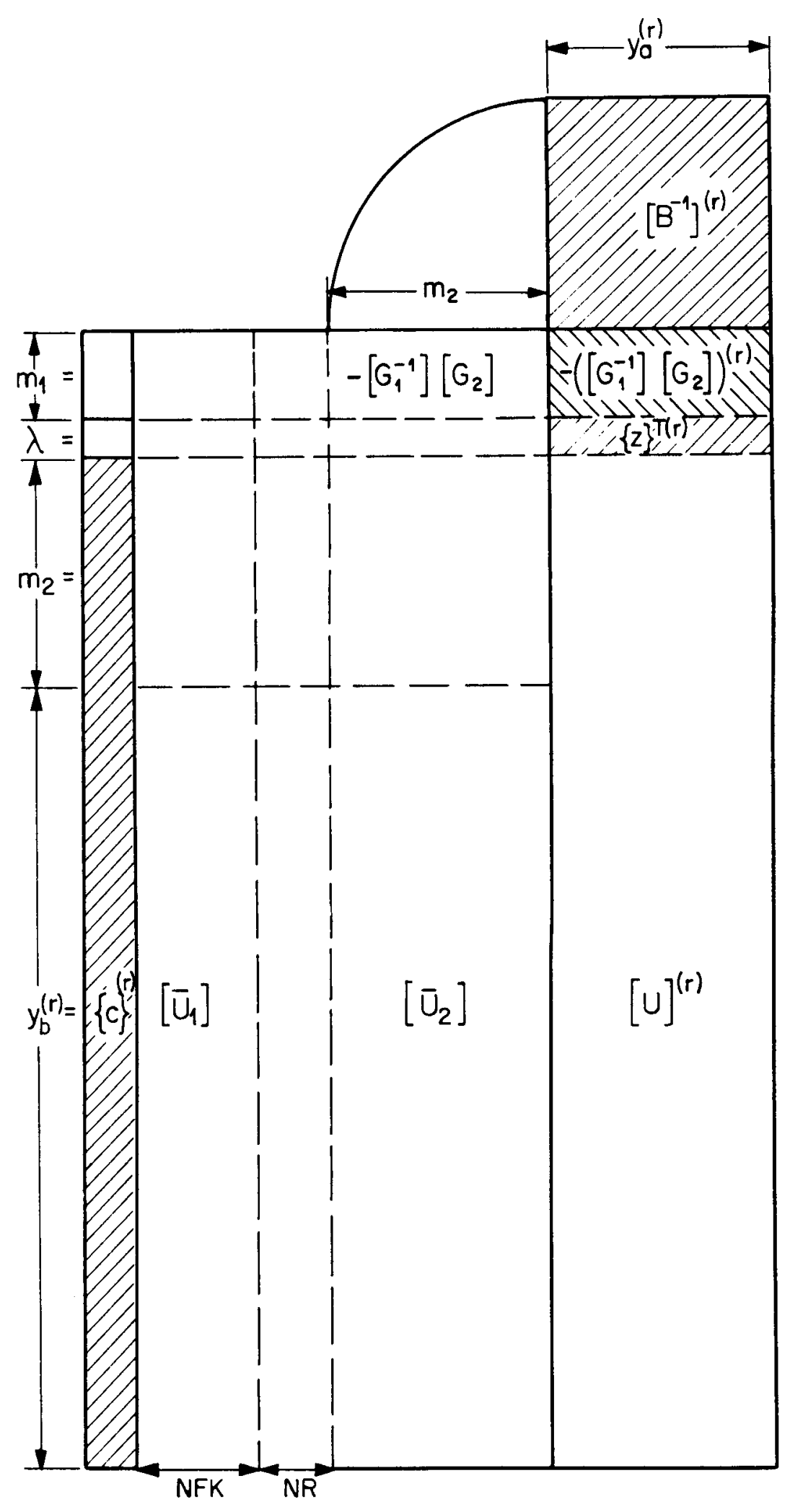

Tableau 7.7 : Optimierung mit dem revidierten Simplexverfohren. 


\section{ANWENDUNG AUF PRAKTISCHE BEISPIELE}

8.1 Computerprogramme

Mit den Computerprogrammen sollen einerseits die Zweckmässigkeit der Ansätze nachgewiesen werden und andererseits praktische Berechnungen wirtschaftlich durchgeführt werden können. Die Programme IRIS-L für das Modell "TLL" (Kapitel 4) und IRIS-C für das Modell "TLC" (Kapitel 5) sind - mit Ausnahme des Programmes für die Benutzung von Grossraumspeichern, für das die Assemblersprache verwendet wurde - in CDC 6400/6500 FORTRAN IV geschrieben. IRIS ist die Abkürzung für "ideal-plastic reinforced-concrete slab solver". Die Programme wurden am Rechenzentrum der Eidg. Technischen Hochschule Zürich (RZETH) geprüft. Sie sind operativ und können für ingenieurmässige Berechnungen verwendet werden. Die beiden Programme haben eine Overlay-Struktur mit dem Problem angepasster Speicherplatzorganisation und Zwischenspeicherung mehrfach benutzter Datenmengen in Grossraumspeichern.

Die Einteilung der Programme in Blöcke ist im Bild 8.1, die Unterteilung des aufwendigsten Blockes (III) im Bild 8.2 dargestellt.

\begin{tabular}{|c|c|c|}
\hline I & $\begin{array}{l}\text { Eingabe und Echoprint } \\
\text { interne Numerierung } \\
\text { Gleichgewichtsmatrix und Lostvektoren } \\
\text { Randbedingungen }\end{array}$ & Tabieau \\
\hline II & Elimination der Gleichungen & Tableau \\
\hline III & $\begin{array}{l}\text { Elimination der freien Voriablen } \\
\text { Optimierung }\end{array}$ & Tableau \\
\hline IV & $\begin{array}{l}\text { optimaler Sponnungszustand } \\
\text { Mechanismus } \\
\text { Ausgabe }\end{array}$ & Tobleau \\
\hline
\end{tabular}


Wahl der Variable, die neu in die Basis kommt Wahl der Variable, welche die Basis verlässt Neve inverse Bosismatrix Neue Zielfunktion und Konstantenvektor

\section{Bild 8.2: Wesentliche Operotionen von Block III}

Die notwendigen Eingabedaten für eine Berechnung sind:

(a) Grösse des Problems

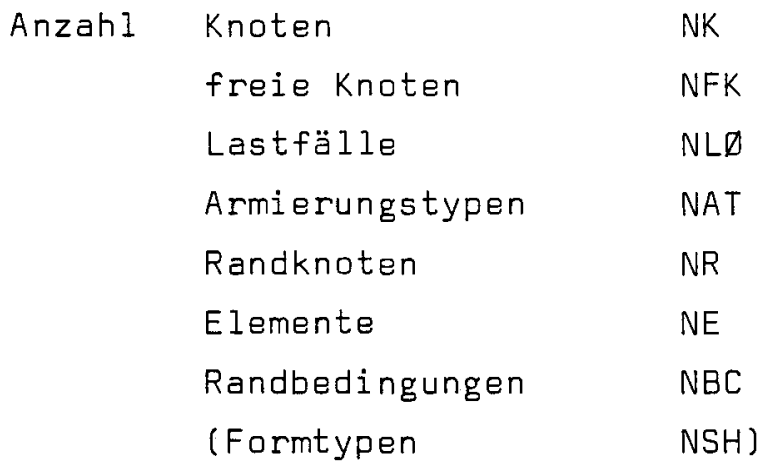

Die Anzahl Seiten kann aus NE und NR berechnet werden:

$$
N S=(3 * N E+N R) / 2
$$

Die Anzahl Formtypen ist nur bei IRIS-C einzugeben; sie gibt an, wieviele Matrizen $\left[U_{n}^{q}\right]$ gemäss Gleichung (5.11) benötigt werden.

(b) Geometrische Angaben und Topologie

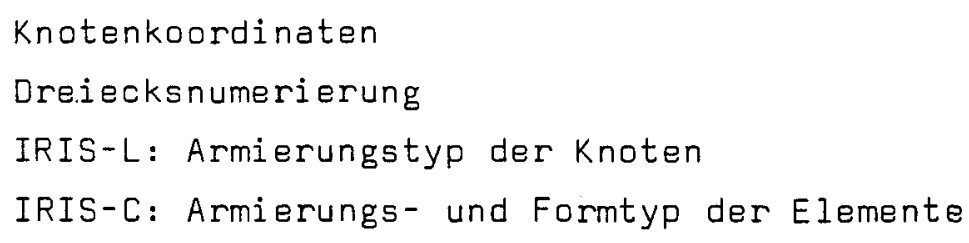

(c) Randbedingungen (RB)

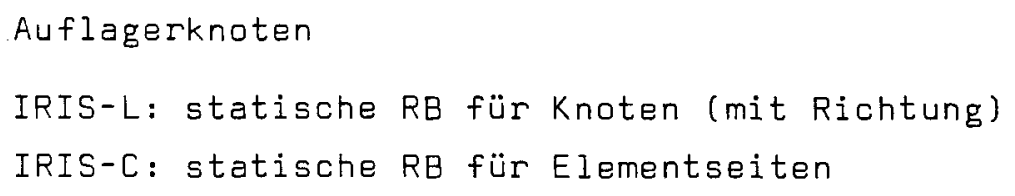


(d) Armierungstypen und Belastung

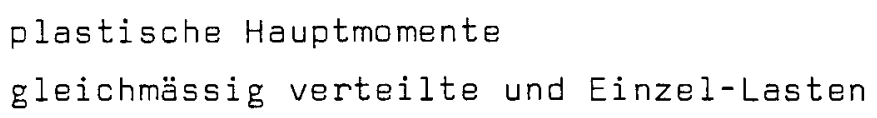

Die Resultate der Berechnung sind:

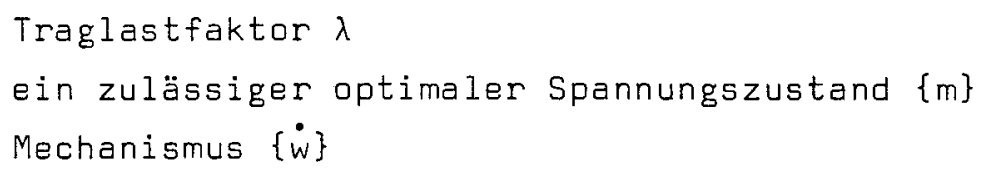

Die Rechenzeit des Zentralrechners für grössere Probleme beträgt bei beiden Programmen etwa

$$
{ }^{t} \mathrm{CP} \cong(0,4 \div 0,7) \cdot 10^{-4} \mathrm{NNB}^{3} \quad(\mathrm{sec})
$$

Dabei ist NNB die Dimension der inversen Basismatrix:

$$
\begin{array}{ll}
\text { IRIS-L: } & \text { NNB }=3 * N K+1-N F K-N B C \\
\text { IRIS-C: } & \text { NNB }=N S+1-N F K-N B C
\end{array}
$$

Der erforderliche Speicherplatz kann mit den unter (a) eingegebenen Grössen vor der Berechnung ermittelt werden.

\subsection{Eingespannte Quadratplatte}

Die Traglast der eingespannten Quadratplatte wird für gleichmässig verteilte Belastung berechnet. Aus Symmetriegründen muss nur ein Achtel der Platte betrachtet werden.

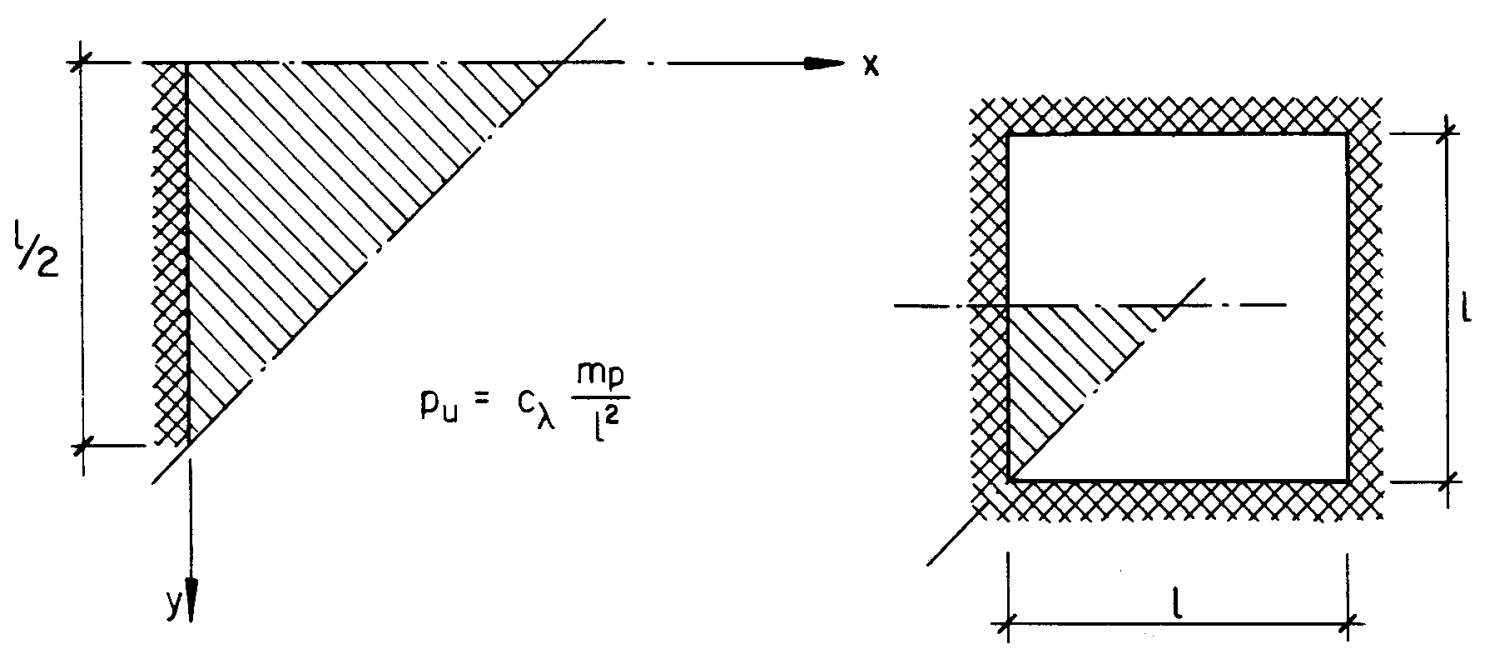

Bild 8.3: Gleichmössig belastete Quadratplatte 
Der exakte Wert der Traglast konnte noch nicht berechnet werden. Als oberer Grenzwert werden in der Literatur [2], [4], [8] je nach gewähltem Mechanismus und Fliessbedingung die Werte $c_{\lambda}=48, c_{\lambda}=43,5$ und $c_{\lambda}=42,9$ angegeben. Der früher in [2] angegebene Wert $c_{\lambda}=37,7$ kann kein oberer Grenzwert sein. Als unteren Grenzwert berechnete Wolfensberger [1] mittels eines vereinfachten Gleichgewichtsmodelles $c_{\lambda}=37,4$.

Die Resultate für $c_{\lambda}$ sind im Bild 8.4 aufgezeichnet. Das Modell "TLL" weist stückweise Iineare Momente, das Modell "TLC" elementweise konstante Momente auf. Man sieht, dass schon bei relativ wenigen Elementen eine für die Praxis

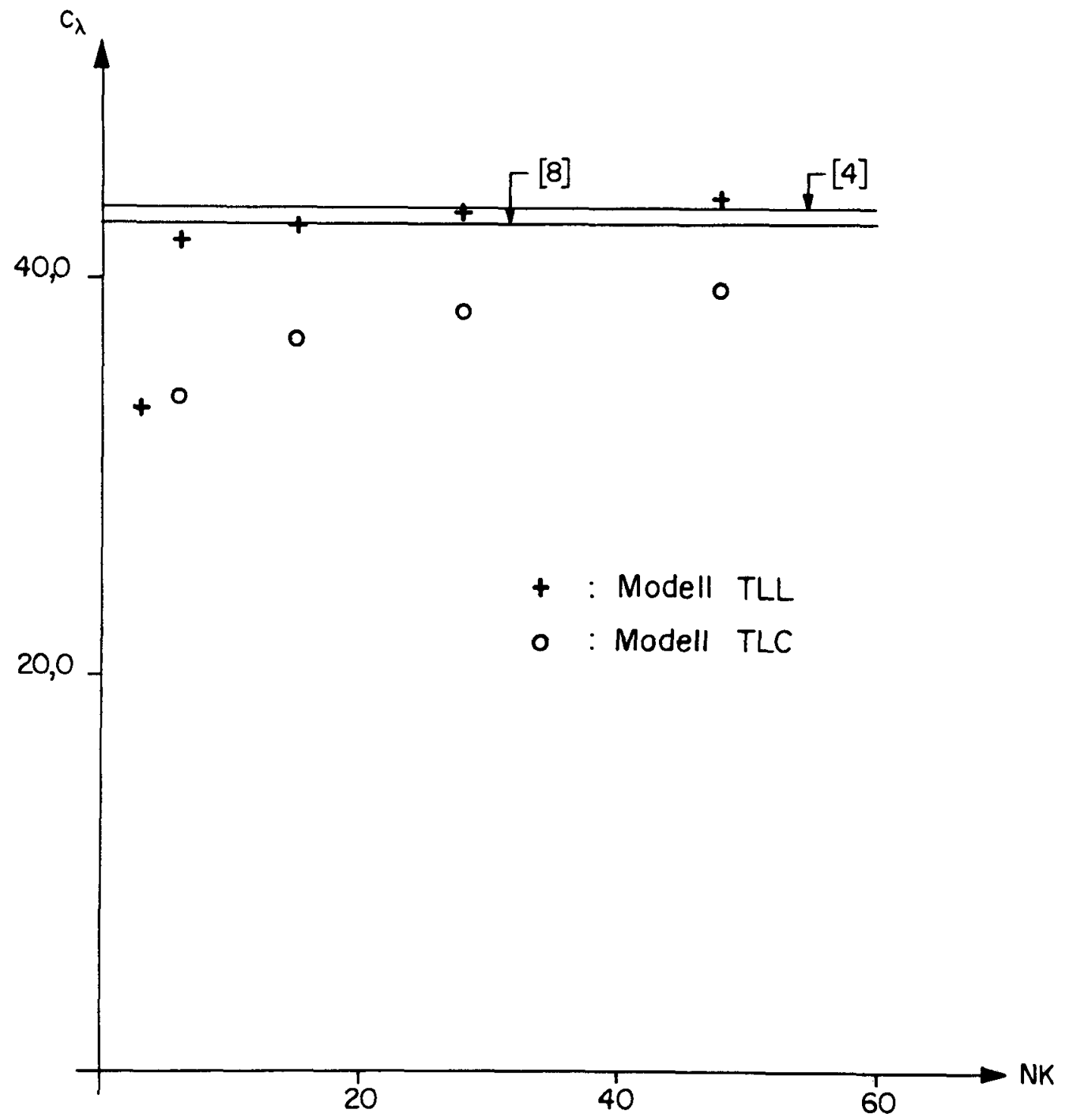

Bild 8.4: Eingespannte Quadratplatte mit konstanter Armierung und Belostung 
genügende Genauigkeit erreicht wird. Die zugehörigen Gleichgewichtslasten haben gezeigt, dass das lokale Gleichgewicht beim Modell "TLL" verletzt wird. Dieses Modell konvergiert nicht monoton gegen einen obern oder untern Grenzwert. Das Modell "TLC" gibt einen untern Grenzwert. Die linearisierte Fliessbedingung bedingt einen zu tiefen Wert. Die Konvergenz ist langsamer als beim Iinearen Ansatz für die Momente.

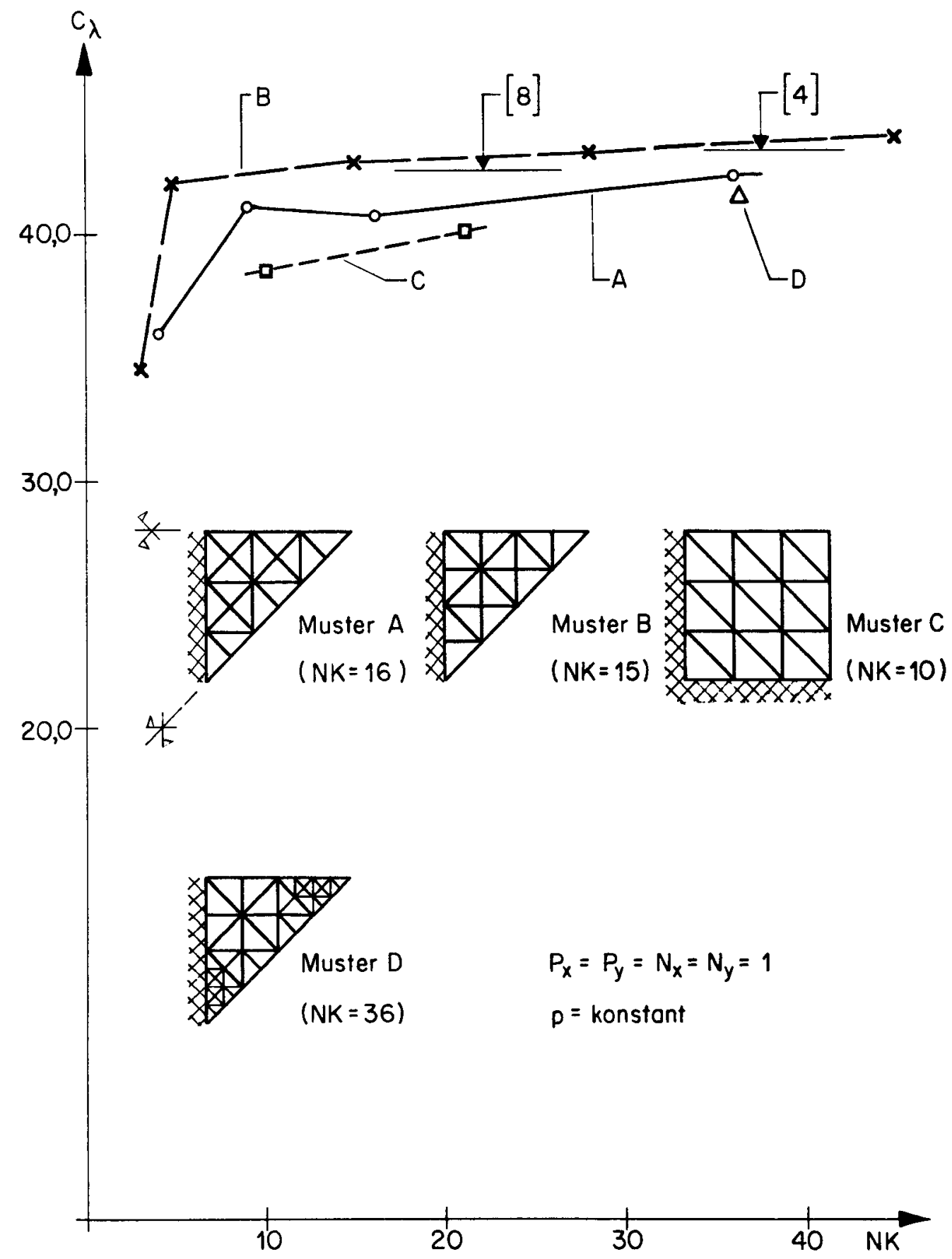

Bild 8.5: Traglasten der eingespannten Quadrotplatte für verschiedene Elementeinteilungen (IRIS-L) 
Der Einfluss der Einteilung in Elemente ist im Bild 8.5 aufgezeichnet. Die Gleichgewichtsbedingungen erfordern (vor allem beim Modell "TLC") eine gewisse Anzahl Elemente, damit der Spannungszustand mit genügender Genauigkeit approximiert werden kann. Der Mechanismus wird stückweise linear approximiert. Es ist daher günstig, wenn die Einteilung so gewählt wird, dass sie dem vermutlichen Mechanismus nicht widerspricht (Bild 8.6).
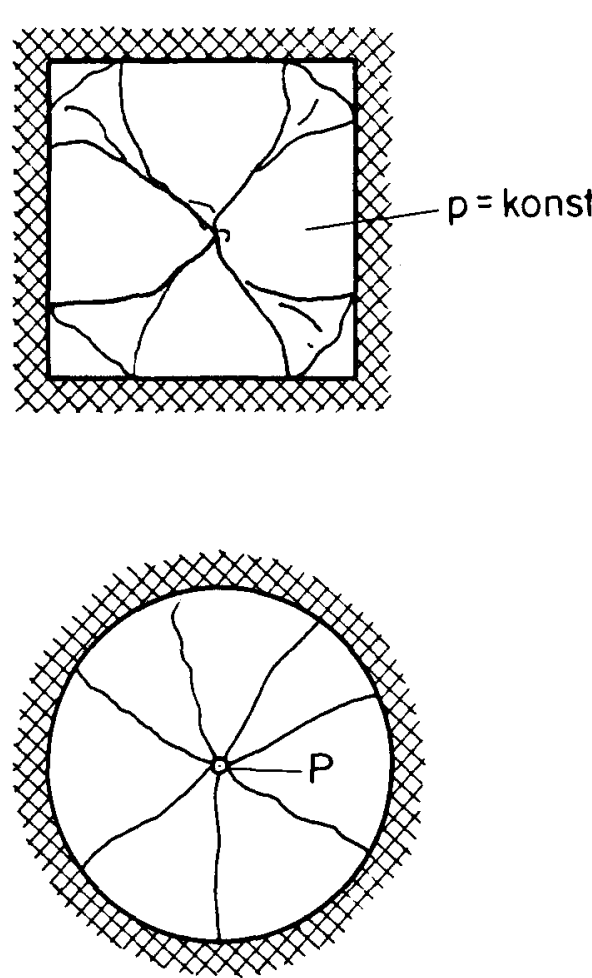

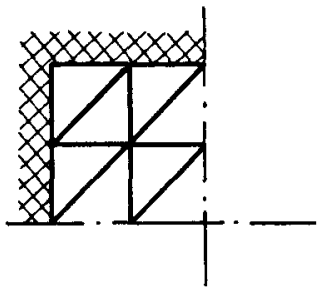

schlecht

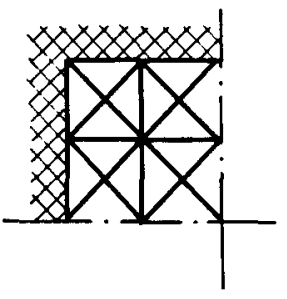

gut

\section{Bild 8.6: Wahl der Einteilung in Elemente}

Eine exakte Lösung ist bei den bekannten kegelförmigen Fächermechanismen nicht zu erreichen, weil Zustände konstanter krümmung nicht mäglich sind. (Für die allseitig frei gelagerte Quadratplatte mit gleichmässiger Belastung resultiert das exakte Resultat $c_{\lambda}=24,0$, wenn die Diagonalen mit Elementseiten zusammenfallen.)

\subsection{Kreisplatte}

Es werden eingespannte und frei aufgelagerte kreisplatten mit konstantem plastischem Moment betrachtet. Die Traglast für gleichmässig verteilte Last und diejenige für eine Einzellast in der Mitte sind bekannt [2], [4]. Die mittels der beiden Modelle berechneten Resultate sind im Bild 8.7 eingetragen. Sie wurden für ein Kreissegment (Symmetrie) von $\alpha \cong 53^{\circ}$ $(\tan \alpha / 2=0,5)$ bestimmt. 


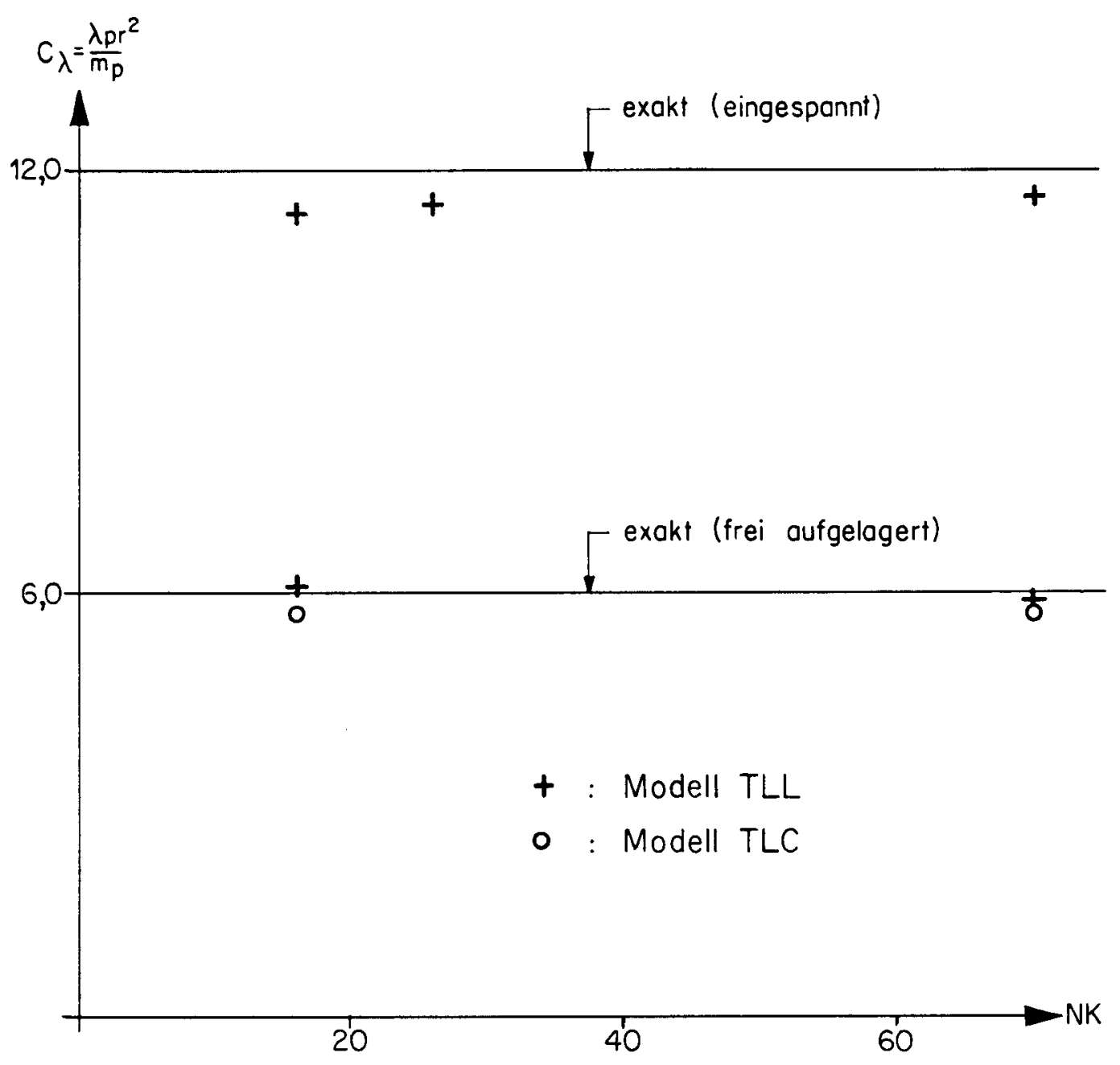

Bild 8.7: Kreisplatte mit konstanter Armierung und Belastung

\subsection{Die unendlich ausgedehnte Flachdecke}

Es wird eine Platte mit konstanter Armierung und Belastung berechnet. Wood [2] hat mittels der Mechanismusmethode für die punktgestützte Flachdecke mit quadratischem Raster gezeigt, dass ein Fächermechanismus mit $r=0$ den obern Grenzwert

$$
p_{u}=4 \pi \frac{m p}{l^{2}}=12,57 \frac{m_{p}}{l^{2}}
$$

hat. (Der gleiche Wert ergibt sich für eine Einzellast auf einer einge- 
spannten Platte, wobei der Radius des Fächermechanismus keine Rolle spielt.) Der mittels finiter Elemente (Bild 8.8 ) berechnete Wert

$$
P_{u} \cong 11,6 \frac{m_{p}}{l^{2}}
$$

stimmt mit dem Resultat (8.1) gut überein.

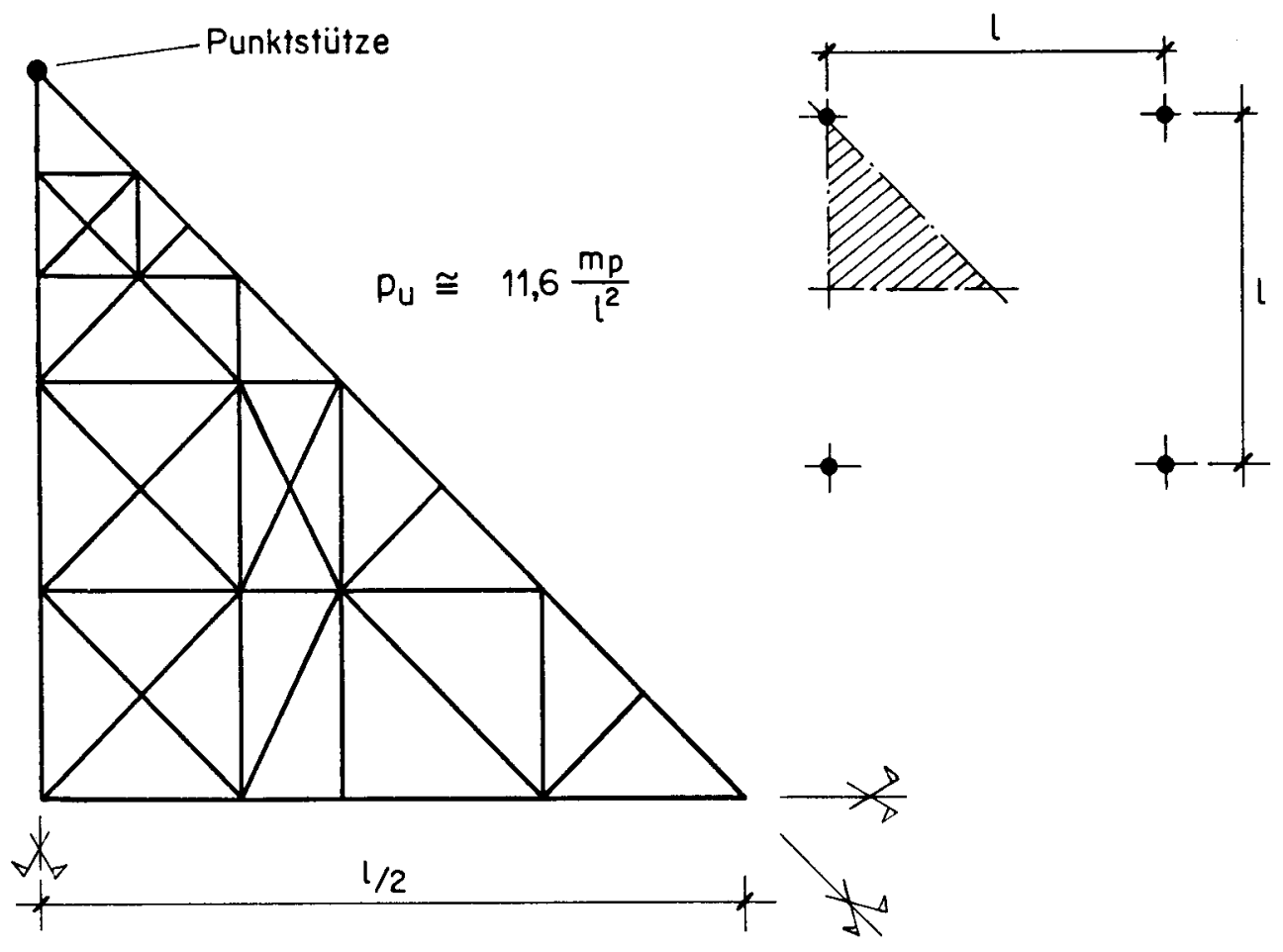

Bild 8.8: Flachdecke mit punktförmigen Stützen

Wolfensberger [1] hat für die Flachdecke auf quadratischen Stützen mit einem Querschnitt $F=\left(\frac{1}{7} 1\right)^{2}$ den untern Grenzwert von

$$
p_{u} \cong 13,0 \frac{m_{p}}{l^{2}}
$$

erhalten. Die Berechnung mit dem Modell "TLL" (Bild 8.9) ergab den Wert

$$
P_{u} \cong 18,9 \frac{m_{p}}{l^{2}}
$$




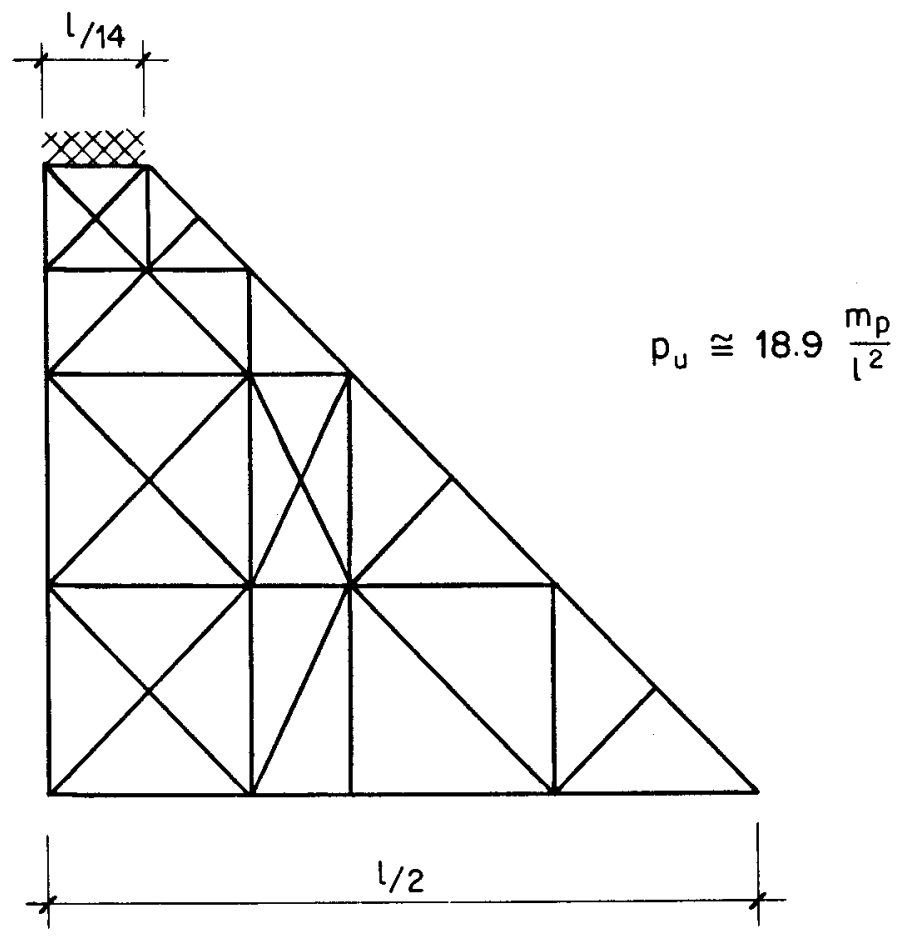

Bild 8.9: Flochdecke ouf Stützen mit $F=\left(\frac{1}{7} \mid\right)^{2}$

Die Einspannmomente der Stütze wurden vernachlässigt. Die berechnete Traglast ist etwas kleiner als diejenige für den im Bild 8.10 dargestellten einfachen Mechanismus.

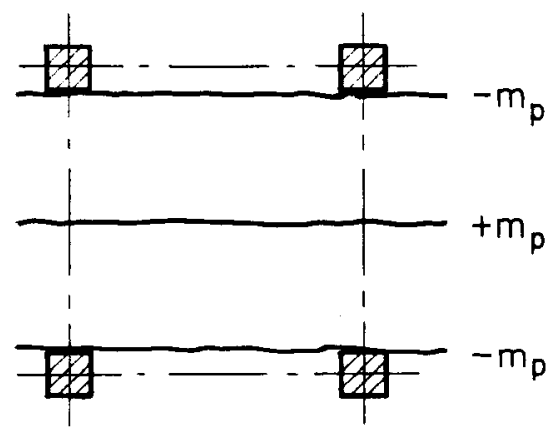

$$
p_{u}=\frac{16 m_{p}}{\left(\frac{6}{7} l\right)^{2}}=22,2 \frac{m_{p}}{l^{2}}
$$

Bild 8.10: Linienmechanismus für Flachdecke

Der mittels finiter Elemente berechnete Mechanismus ist im Bild 8.11 dargestellt. 


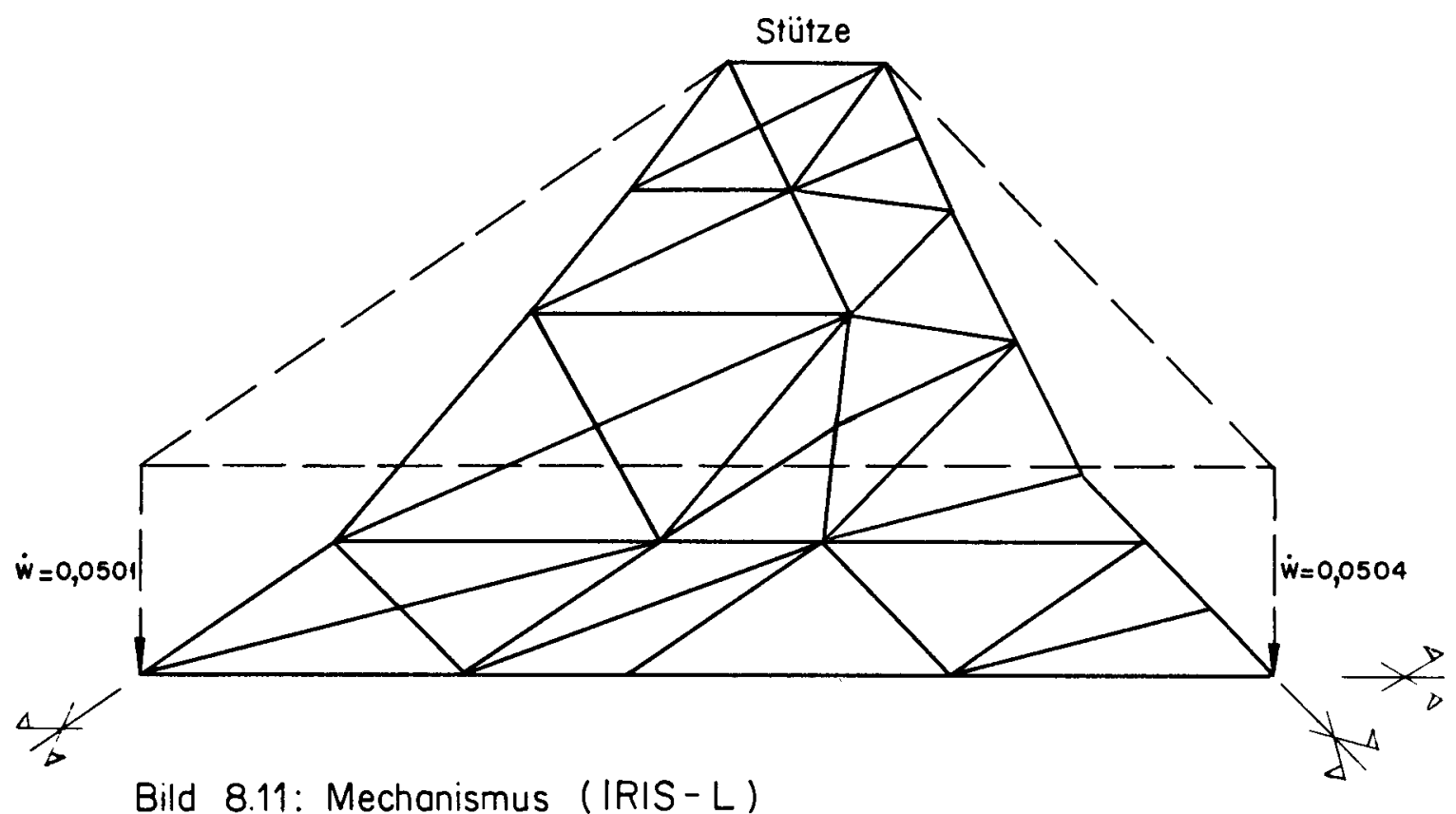

Bild 8.11: Mechanismus (IRIS-L)

\subsection{Orthotrope, schiefe Armierung}

Als Beispiel wurde die von Wood [2, S. 133] berechnete Quadratplatte mit gleichmässig verteilter Belastung verwendet. Die Platte ist auf drei Seiten frei drehbar gelagert; die vierte Seite ist frei (Bild 8.12).

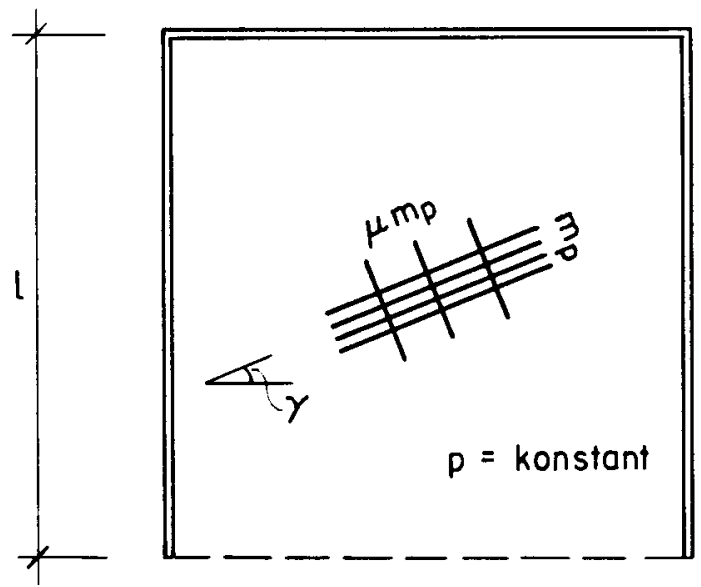

Numerische Werte

ton $\gamma=0,4$

$m_{p}=P_{1}=300,0$

$\mu=0,5$

Bild 8.12: Quadratplatte mit schiefer Armierung 
Die Auswertung der analytischen Ausdrücke [2] ergibt

$$
\begin{aligned}
\xi & =0,611 \\
Y & =0,458 \\
\lambda p l^{2} & =11,63 m_{p}=3490
\end{aligned}
$$

als obern Grenzwert der Traglast. Nach Sawzuk-Jaeger [18, S. 324] ergibt sich

$$
\begin{gathered}
\frac{q^{b}}{M_{0}} \cong 20 \\
\lambda p^{2} \cong 3400
\end{gathered}
$$



\section{Bild 8.13: Einteilung in Elemente}

Das Modell "TLL" mit der im Bild 8.13 aufgezeichneten, relativ groben Einteilung in Elemente ergab das Resultat

$$
\lambda p l^{2} \cong 3200
$$

Dabei ist zu beachten, dass entlang des aufgelegten Randes eine obere Armierung nötig ist, weil sonst kein Drillungsmoment aufgenommen werden kann. 
platzbedarf auswirkt. Die Anzahl Knoten ist NK = 25, die Anzahl freier Knoten NFK = 12. Es wurden infolge Randbedingungen total 20 Momentenparameter null gesetzt: $N B C=20$. In der untenstehenden Tabelle sind die Matrizen, die Gleichungen, in denen sie definiert sind und die Dimensionen angegeben.

\begin{tabular}{lccc} 
Matrix & Gleichung & \multicolumn{2}{c}{ Dimension } \\
& & \multicolumn{2}{c}{ mit } \\
& & $12 \times 75$ & $12 \times 75$ \\
{$\left[G_{f}\right]$} & $(4.5)$ & $12 \times 55$ & $12 \times 75$ \\
{$\left[G_{x-y}\right]$} & $(4.20)$ & $12 \times 55$ & $12 \times 75$ \\
{$[G]$} & $(4.28)$ & $12 \times 44$ & $12 \times 64$ \\
{$\left[G_{2}\right]$} & $(7.2)$ & $44 \times 44$ & $64 \times 64$ \\
{$[B]^{-1}$} & $(7.5)$ & &
\end{tabular}

Entscheidend für den Platzbedarf ist die inverse Basismatrix $[B]^{-1}$. Deren Koeffizienten müssen alle gespeichert und in der Regel bei jedem Austauschschritt neu berechnet werden. Die Umgruppierung der Momentenparameter bewirkt, dass sie etwa 2,1 mal kleiner wird. 
ANHANG A

Tatsächliches Verhalten von Stahlbetonplatten

Das wirkliche Verhalten von Stahlbetonplatten kann nur durch Versuche ermittelt werden. Die Abweichungen der Versuchsresultate ergeben sich - wenn man die Abweichungen infolge der Konstruktion der Versuchsanlage, die Ungenauigkeiten der Versuchskörper und die Messungenauigkeiten vernachlässigen kann - aus den im Modell vorgenommenen Idealisierungen.

Die bei den verwendeten Modellen angenommenen physikalischen Vereinfachungen sind:

A1. Starr-plastisches Verhalten: Die elastischen Deformationen und die Verfestigung des Materials werden nicht berücksichtigt (Fliessplateau).

A2. Johansen'sche Fliessbedingung: Wenn die Tragfähigkeit erschöpft ist, fliessen alle Armierungsstähle, die den plastifizierten Schnitt kreuzen.

A3. Plattentheorie 1. Ordnung: Die Normalen zur Mittelebene bleiben gerade und senkrecht zur Mittelebene, die Spannungen in der Mittelebene sind null (keine Membrantragwirkung).

Die mathematischen Vereinfachungen der beiden Modelle sind:

A4. Linearisierung der Fliessbedingungen: Die Tragfähigkeit wird ohne physikalischen Grund herabgesetzt.

A5. Diskretisation: Das Gleichgewicht wird lokal verletzt (Modell "TLL"); die Belastung wird durch Einzellasten in den Knoten ersetzt (Modell "TLC").

Ferner wird vorausgesetzt, dass kein Schubbruch eintritt (ausreichende Schubtragfähigkeit). Die äussere Belastung wird als bekannt angenommen. Schliesslich müsste die Frage gestellt werden, wie weit die Traglast als Bemessungskriterium geeignet ist [15]. Dieses Problem wird hier nicht weiter untersucht.

Während der Einfluss mathematischer Vereinfachungen durch mathematische Methoden erfasst werden kann (z.B. Verfeinerung der approximativen Fliess- 
bedingung, Verfeinerung der Einteilung in finite Elemente, sind die physikalischen Idealisierungen durch Versuche zu überprüfen.

Bei den heute vorliegenden Versuchen wurde vor allem die vereinfachende Annahme des starr-plastischen Verhaltens oder eines elastisch-plastischen Verhaltens ohne Beschränkung durch die Rotationsfähigkeit untersucht. Die nicht linearisierte Fliessbedingung (Gleichung (2.13)), die sich aus dieser Annahme ergibt, hat sich bei Versuchen mehrfach bestätigt [13], [14]. Die Mitwirkung von flach zur Bruchlinie liegenden Armierungsstäben wurde in [10] in Frage gestelit. Die anderen Experimente zeigen jedoch, dass bei Platten, die nicht überarmiert sind (Betonstauchung nicht massgebend), keine wesentliche Abminderung durch nicht fliessende Armierungsstäbe zu befürchten ist.

Platten ohne obere Armierung können nach der hier verwendeten Fliessbedingung im plastifizierten Schnitt keine Drillungsmomente übernehmen. Bei Versuchen an frei aufliegenden Platten [18] ist jedoch die daraus folgende Abminderung der Traglast nicht beobachtet worden. Es ist möglich, dass die Drillungsmomente durch die Zugfestigkeit des Betons aufgenommen wurden.

Dem Durchstanzen ist besonders bei Einzellasten und Stützen Beachtung zu schenken. Es stellt sich bei der praktischen Bemessung die Frage, wie weit es möglich ist, dieses Problem unabhängig vom Tragverhalten auf Biegung und Drillung zu behandeln. Es scheint, dass weitere experimentelle Untersuchungen einer analytischen Formulierung vorangehen müssten. 
Im ersten Teil dieses Anhanges wird das Gleichgewicht des Modelles "TLL" untersucht, im zweiten Teil dasjenige des Modelles "TLC". In beiden Fällen ist die Gleichgewichtsgleichung (4.18) zu integrieren. Die Integranden sind unstetige Funktionen.

Beim Modell "TLL" können die Unstetigkeiten (Pole) im Ausdruck (4.18) durch partielle Integration nach dem Green'schen Integralsatz

$$
\iint A \frac{\partial^{2} B}{\partial x_{i} \partial x_{j}} d x_{i} d x_{j}+\iint \frac{\partial A}{\partial x_{i}} \frac{\partial B}{\partial x_{j}} d x_{i} d x_{j}=\oint A \frac{\partial B}{\partial x_{j}} \nu_{i} d s
$$

behoben werden. Man erhält

$$
\begin{aligned}
& -\oint_{C}\left(m_{x} w_{, x}^{*} v_{x}+m_{x y} w_{, y}^{*} v_{x}+m_{x y} w_{, x}^{*} \nu_{y}+m_{y} w_{, y}^{*} v_{y}\right) d s \\
& +\iint_{F}\left(m_{x, x} w_{, x}^{*}+m_{x y, x} w_{, y}^{*}+m_{x y, y} w_{, x}^{*}+m_{y, y} w_{, y}^{*}\right) d x d y \\
& -\lambda \iint_{F} p w^{*} d x d y=0
\end{aligned}
$$

Die Winkelfunktionen $v_{x}$ und $\nu_{y}$, sowie der Rand $C$ sind im Bild B.1 angegeben. Der Vektor $\{\nu\}$ hat die Länge 1 .

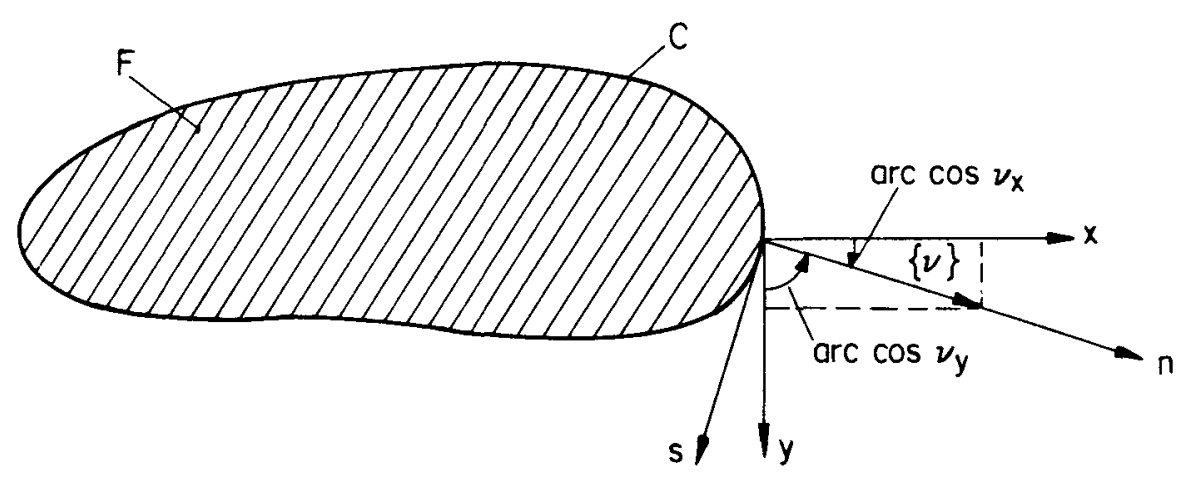

Bild B.1: Definition der Randwerte 
Das Integral über den Rand C kann noch mittels der Beziehungen

$$
\begin{aligned}
& m_{n}=m_{x} \nu_{x}^{2}+m_{y} \nu_{y}^{2}+2 m_{x y} \nu_{x} \nu_{y} \\
& m_{n s}=m_{y} \nu_{x} \nu_{y}-m_{x} \nu_{x} \nu_{y}+m_{x y} \nu_{x}^{2}-m_{x y} \nu_{y}^{2} \\
& w_{, n}^{*}=w_{, x}^{*} \nu_{x}+w_{, y}^{*} \nu_{y} \\
& w_{, s}^{*}=-w_{, x}^{*} \nu_{y}+w_{y}^{*} \nu_{x}
\end{aligned}
$$

umgeformt werden in

$$
-\oint_{C}\left(m_{x} w_{, x}^{*} v_{x}+\cdots \cdots\right) d s=-\oint_{C}\left(m_{n} w_{, n}^{*}+m_{n s} w_{, s}^{*}\right) d s
$$

Dieses Integral kann ausgewertet werden, weil am Rand $m_{n}$ oder $w^{*}, n$ und $m_{n s}$ oder ${ }^{*}{ }^{*}$ s kontinuierlich sind.

Das zweite Integral in der Gleichung (B.2) kann elementweise integriert werden

$$
\begin{array}{ll}
\iint_{F}\left(m_{x, x} w_{, x}^{*}+\cdots \cdots\right) d F= & \sum_{e} \iint_{F^{e}}\left(m_{x, x} w_{, x}^{*}+\cdots\right) d F^{e} \\
=\sum_{e} \iint_{F} m_{i j, j}^{e} w_{, i}^{* e} d F^{e} & (i=x, y ; j=x, y)
\end{array}
$$

weil die Integration über die Elementgrenzen keinen Beitrag ergibt:

$$
\lim _{\epsilon \rightarrow 0} \int_{\xi=-\epsilon}^{\xi=+\epsilon} m_{\xi, \xi} w_{, \xi}^{*} d \xi d \eta=0
$$

Die Gleichung (B.5) wird im Bild B.2 veranschaulicht. Hätte der Integrand in der Gleichung (B.5) einen Pol, so wäre der Grenzwert nicht null. 

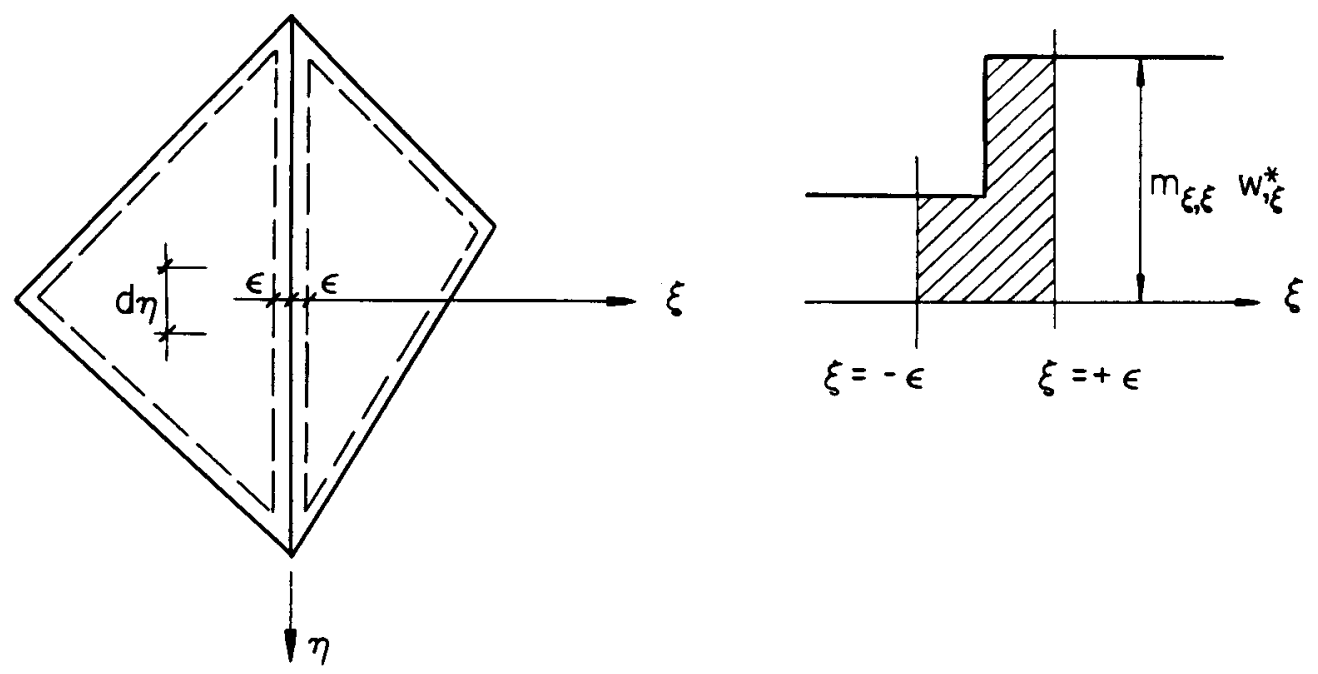

Bild B.2: Integration über Elementgrenzen

Die Integrale auf der rechten Seite der Gleichung (B.4) können mittels des Green'schen Integralsatzes wieder zurücktransformiert werden zu

$$
\iint_{F^{e}} m_{i j, j}^{e} w_{, i}^{* e} d F^{e}=\oint_{c^{e}}\left(m_{n}^{e} w_{, n}^{* e}+m_{n s}^{e} w_{, s}^{* e}\right) d s^{e}-\iint_{F^{e}} m_{i j}^{e} w_{, i j}^{* e} d F^{e} \quad \text { (B.6) }
$$

Das letzte Integral verschwindet, weil innerhalb der Elemente überall ${ }^{w}{ }^{*}{ }_{i j}=0$ ist. Der mittlere Ausdruck kann nur ausgewertet werden, weil am Rand des Elementes $m_{n}$ oder $w^{*}, n$ und $m_{n s}$ oder $w^{*}{ }^{*}$ s kontinuierlich sind. Diese Kontinuitätsbedingungen wurden schon im Abschnitt 3.2 erwähnt. Man stellt fest, dass die in diesem Modell vorkommende kontinuität von $m_{n s}$ nicht nötig wäre, weil $w^{*}, 5$ kontinuierlich ist.

Aus den Gleichungen (B.2), (B.3) und (B.6) ergibt sich

$$
-\oint_{C}\left(m_{n} w_{, n}^{*}+m_{n s} w_{, s}^{*}\right) d s+\sum_{e} \oint_{C}\left(m_{n}^{e} w_{, n}^{* e}+m_{n s}^{e} w_{, s}^{* e}\right) d s^{e}-\lambda \iint_{F} p w^{*} d F=0
$$

Entlang der inneren seiten heben sich die Beiträge $m_{n s^{e}}{ }^{*}{ }^{*}$ s der anschliessenden Elemente $(+)$ und $(-)$ im Bild $B .3$ weg, weil $m_{n s}^{e^{+}}=m_{n s} e^{e^{s}}$ und $w^{*} e^{+}=-w^{*} e^{-}$ ist. 


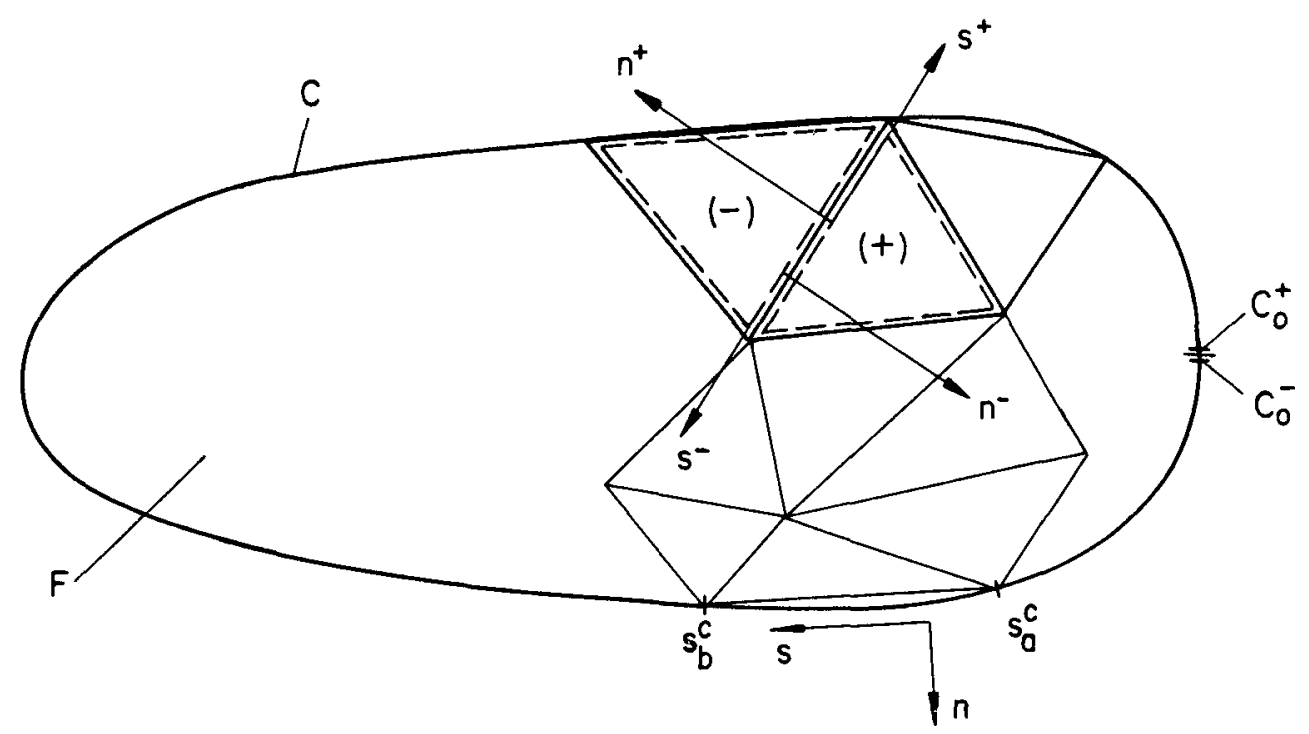

Bild B.3: Integrationsbereiche

Entlang der Randseiten werden die Beiträge $m_{n s}^{e}{ }^{*}{ }^{*}, s$ durch die Integrale

$$
-\oint_{C} m_{n s} w_{, s}^{*} d s
$$

aufgehoben, weil $m_{n s}=m_{n s}^{e}$ und aus Kompatibilitätsgründen $w^{*}, s=w^{*}$, s ist. Demgegenüber müssen ${ }^{*}, n$ und ${ }^{*}{ }^{*} e$, nicht übereinstimmen. Weil also $w^{,}, n$ unabhängig variiert werden kann, muss die statische Randbedingung

$$
m_{n}=0, \text { sofern } w_{, h}^{*} \neq 0
$$

erfüllt sein, wenn die Gleichgewichtsgleichung (B.7) gelten soll. Wenn $w^{*}, n \neq 0$, ist der Rand um die Axe $s$ frei drehbar. Für $m_{n s}$ können keine Randbedingungen formuliert werden, weil es nicht mäglich ist, w, unabhängig zu variieren.

Wenn die Randbedingungen erfüllt sind, reduziert sich die Gleichung (B.7) auf

$$
\sum_{e} \oint_{c^{e}} m_{n}^{e} w_{, n}^{* e} d s^{e}-\lambda \iint_{F} p w^{*} d F=0
$$

Unter Berücksichtigung, dass $\omega_{n}^{*}=w^{*}, n^{*}$ ist damit die Gleichung (4.6) für den Beitrag eines Elementes zur innern Arbeit verifiziert. 
Es soll jetzt noch untersucht werden, ob die Gleichgewichtsgleichung (4.18) exakt erfüllt ist. Gegeben ist also ein stückweise lineares Momentenfeld. Es ist anzugeben, ob die äussern Lasten $\lambda p(x, y)$ und die Reaktionen damit im Gleichgewicht stehen. Die sogenannten Gleichgewichtslasten, d.h. diejenigen Lasten, welche mit dem Momentenfeld im Gleichgewicht stehen, werden mit $\lambda \mathrm{p}_{e}(x, y)$ bezeichnet. Das Momentenfeld ist dann mit den äussern Lasten im Gleichgewicht, wenn $\lambda p(x, y)=\lambda p_{e}(x, y)$ ist.

Das Gleichgewicht kann wieder mittels des Prinzipes der virtuellen Verschiebungen formuliert werden:

$$
-\iint_{F} m_{i j} w_{, i j}^{*} d F-\lambda \iint P_{e} w^{*} d F=0
$$

Dabei nehmen $i$ und $j$ wieder die Werte $x$ und $y$ an. Ist die Randbedingung (B.B) erfüllt, so führen die Gleichungen (B.2), (B.3) und (B.4) auf

$$
-\oint_{C} m_{n s} w_{, s}^{*} d s+\sum_{e} \iint_{F^{e}} m_{i j, j}^{e} w_{, i}^{*} d F^{e}-\lambda \iint p_{e} w^{*} d F=0
$$

Wenn man auf die Gleichgewichtslasten kommen will, muss man nochmals partiell integrieren, damit die virtuelle Verschiebung $w^{*}$ anstelle ihrer Ableitungen auftritt.

Die partielle Integration des ersten Ausdruckes der Gleichung (B.11) ergibt

$$
-\oint_{C} m_{n s} w_{, s}^{*} d s=-\left.m_{n s} w^{*}\right|_{C_{0}^{-}} ^{C_{0}^{+}}+\oint_{C} m_{n s, s} w^{*} d s
$$

Der erste Ausdruck auf der rechten Seite verschwindet, weil der Rand geschlossen ist (Bild B.3). Ist der Rand C glatt, so ist der letzte Ausdruck leicht auszuwerten, weil $\mathrm{m}_{\mathrm{ns}}$ kontinuierlich ist. Wenn der Rand Ecken hat, so ist dort $m_{n s, s}=\infty$ und das Linienintegral muss seitenweise berechnet werden:

$$
-\sum_{c} \int_{s_{0}}^{s_{b}} m_{n s}^{c} w_{, s}^{* c} d s^{c}=-\sum_{c}\left(\left.m_{n s}^{c} w^{* c}\right|_{s_{0}^{c}} ^{s_{b}^{c}}-\int_{s_{0}^{c}}^{s_{b}^{c}} m_{n s, s}^{c} w^{* c} d s^{c}\right)
$$

Die Integrationsgrenzen $s_{a}^{c}$ und $s_{b}^{c}$ sind im Bild B.3 angegeben; der Index c läuft über alle Dreiecksseiten auf dem Rand $C$. 
gibt

$$
\sum_{e} \int_{F^{e}} m_{i j, j}^{e} w_{, i}^{* e} d F^{e}=\sum_{e}\left(\oint_{C^{e}} m_{i j, j}^{e} \nu_{i}^{e} w^{* e} d s^{e}-\iint_{F^{e}} m_{i j, i j}^{e} w^{* e} d F^{e}\right)
$$

Der letzte Ausdruck verschwindet, weil innerhalb des Elementes $m_{i j, i j}$ überall null ist. Der mittlere Ausdruck kann für jede der drei Seiten eines Elementes berechnet werden:

$$
\sum_{e} \underset{c^{e}}{\oint_{i j, j}} m_{i}^{e} w^{* e} d s^{e}=\sum_{e} \sum_{d=1}^{3} \int_{s_{a}^{d}}^{s_{b}^{d}}\left(m_{n, n}^{d}+m_{n s, s}^{d}\right) w^{* d} d s^{d}
$$

Entlang der inneren Seiten heben sich die Beiträge von $m_{n s, s}^{d}{ }^{*} d$ auf, so dass

$$
\sum_{e} \sum_{d} \int_{s_{a}^{d}}^{s_{b}^{d}} m_{n s, s}^{d} w^{* d} d s^{d}=\sum_{c} \int_{s_{0}^{c}}^{s_{b}^{c}} m_{n s, s}^{c} w^{* c} d s^{c}
$$

Weiter können die Beiträge von $m_{n, n}^{d}{ }^{*}{ }^{*}$ über die drei Seiten der Elemente zusammengefasst werden, weil der Integrand in den Ecken der Elemente keine Pole aufweist.

$$
\sum_{e} \sum_{d} \int_{s_{a}^{d}}^{s_{b}^{d}} m_{n, n}^{d} w^{* d} d s^{d}=\sum_{e} \oint_{c} m_{n, n}^{e} w^{* e} d s^{e}
$$

Werden die Gleichungen (B.12) bis (B.17) in die Gleichung (B.11) eingesetzt, so ergibt sich

$-\sum_{c}\left(\left.m_{n s}^{c} w^{* c}\right|_{s_{a}^{c}} ^{s_{b}^{c}}-2 \int_{s_{a}^{c}}^{s_{b}^{c}} m_{n s, s}^{c} w^{* c} d s^{c}\right)+\sum_{e} \oint_{c^{e}} m_{n, n}^{e} w^{* e} d s^{e}-\lambda \iint_{F} p_{e} w^{*} d F=0$

Aus dieser Gleichung sind nun die im Bild 4.10 dargestellten Gleichgewichtslasten ersichtlich.

Das Gleichgewicht (Gleichung (4.18)) kann also für das Modell "TLL" in Form der Normalmomente entlang der Seiten

$$
\sum_{e} \oint_{C^{e}} m_{n}^{e} w_{, n}^{* e} d s^{e}-\lambda \iint_{F} p w^{*} d F=0
$$

oder in Form der Gleichgewichtslasten (Gleichung (B.18)) ausgedrückt werden.

Die Auswertung der Integrale über F, d.h. die Bestimmung der äussern Arbeit $A_{a}^{*}$, ist einfach. Die Berechnung der innern Arbeit $A_{i}^{*}$ soll noch am Beispiel 
der virtuellen Verschiebung des Mittelpunktes einer eingespannten schiefen Platte gezeigt werden.

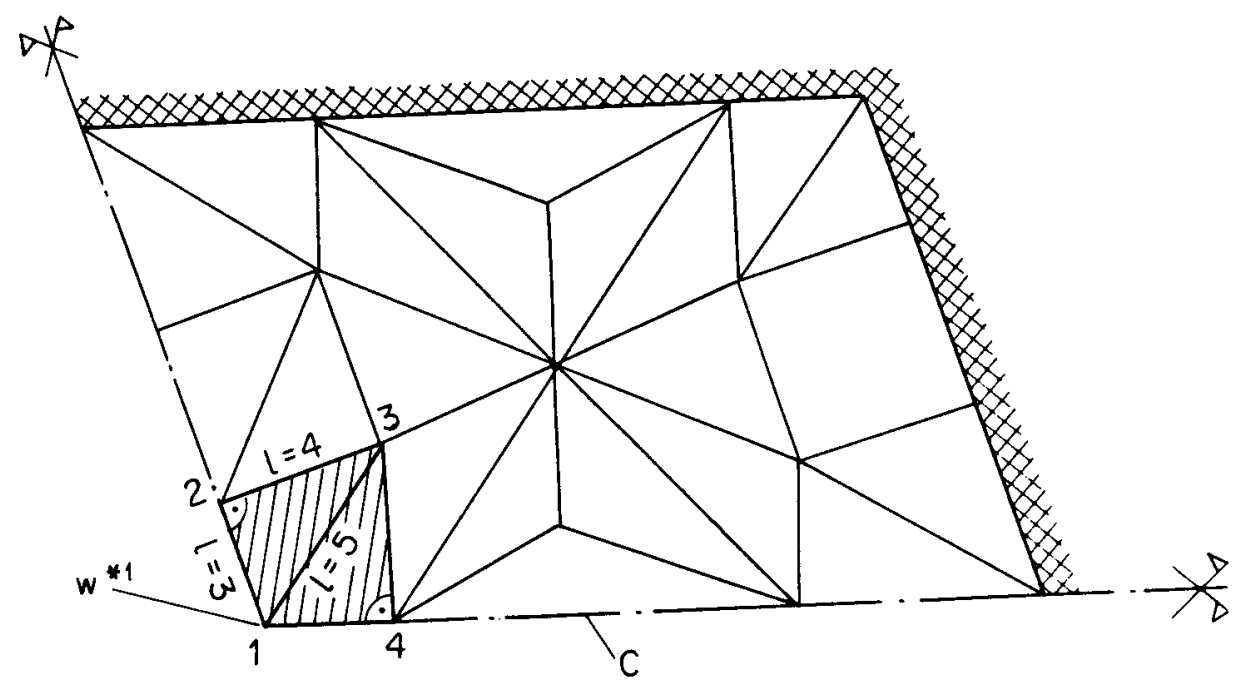

Bild B.4: Schiefe Platte

Es sei folgendes Momentenfeld gegeben.

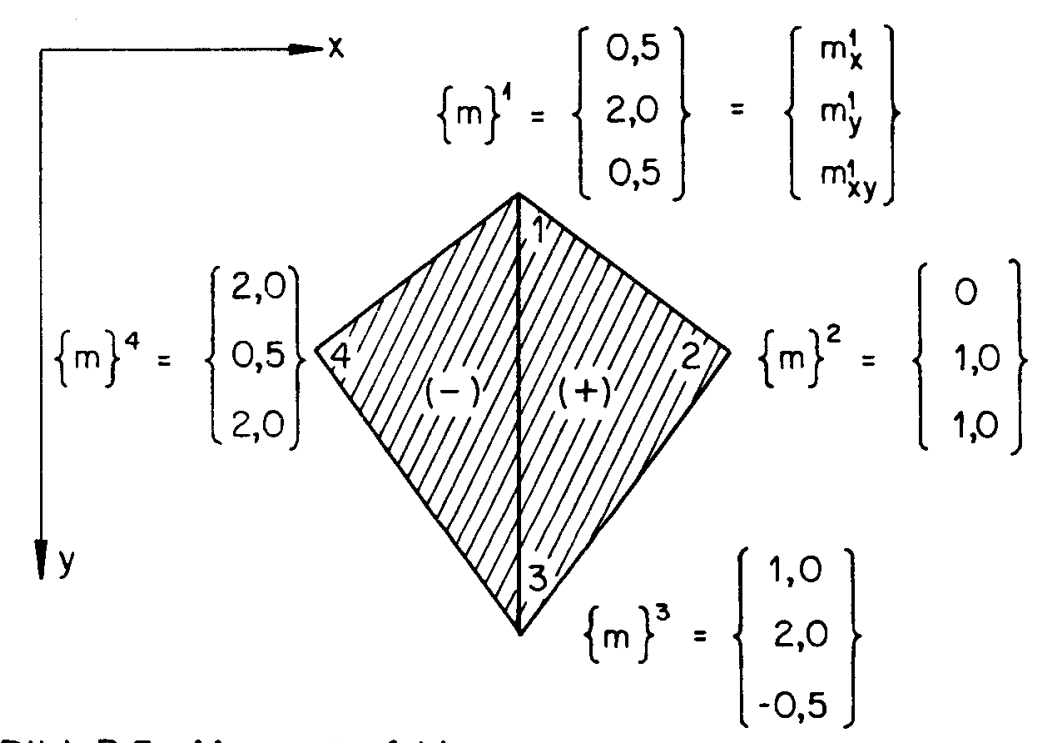

Bild B.5: Momentenfeld

Daraus bekommt man die Normalmomente $m_{n}$ entlang der Seiten üter die Gleichung

$$
m_{n}=m_{x} \cos ^{2} \varphi+m_{y} \sin ^{2} \varphi+2 m_{x y} \sin \varphi \cos \varphi
$$


Sie sind in der untenstehenden Figur zusammengestellt.

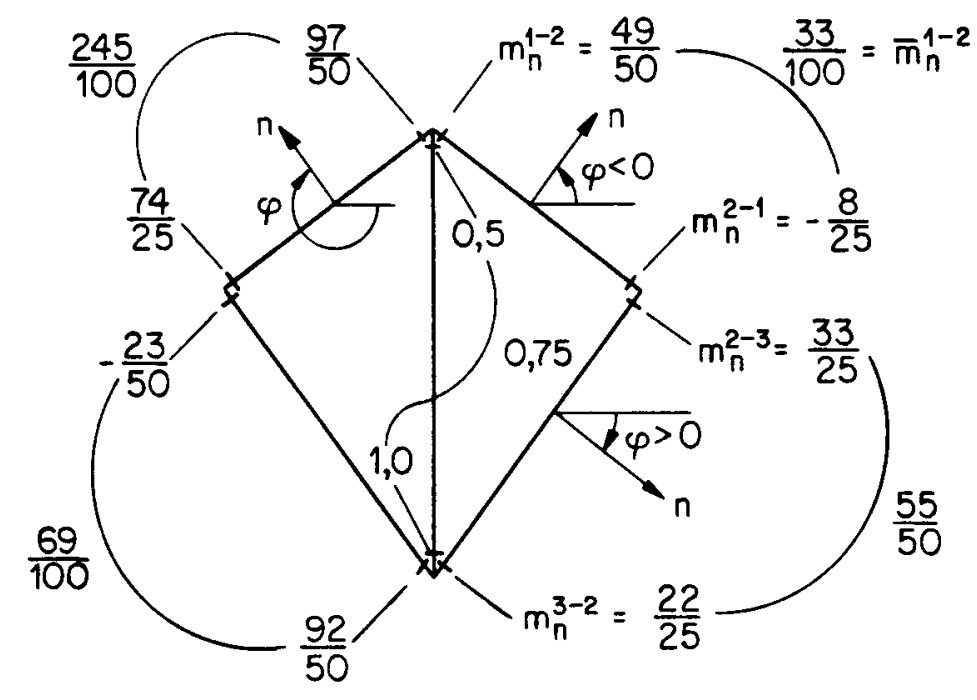

Beispiel: $\quad \sin \varphi^{1-2}=-\frac{4}{5}, \cos \varphi^{1-2}=\frac{3}{5}$

$$
m_{n}^{\prime-2}=0,5 \cdot \frac{9}{25}+2,0 \cdot \frac{16}{25}-1,0 \cdot \frac{12}{25}=\frac{49}{50}
$$

Bild B.6: Momente $m_{n}$

Das erste Integral in der Gleichung (B.9) kann seitenweise ausgewertet werden:

$$
\begin{aligned}
& \int_{1}^{2} m_{n} w_{, n}^{*} d s=\frac{1}{2}\left(m_{n}^{1-2}+m_{n}^{2-1}\right) w_{, n}^{* 1-2} l^{1-2}=\bar{m}_{n}^{1-2} \bar{\omega}_{n}^{* 1-2} \\
& \int_{i}^{3} m_{n} w_{, n}^{*} d s=\frac{1}{2}\left(m_{n}^{1-3}+m_{n}^{3-1}\right)\left(w_{, n}^{*-}+w_{, n}^{*+}\right) l^{1-3}=\bar{m}_{n}^{1-3} \Delta \bar{\omega}_{n}^{* 1-3}
\end{aligned}
$$

Die $\bar{\omega}_{n}^{*}$ und $\Delta \bar{\omega}_{n}^{*}$ werden auf geometrische weise aus den virtuellen Verschiebungen infolge $w^{* 1}=1$ bestimmt. 


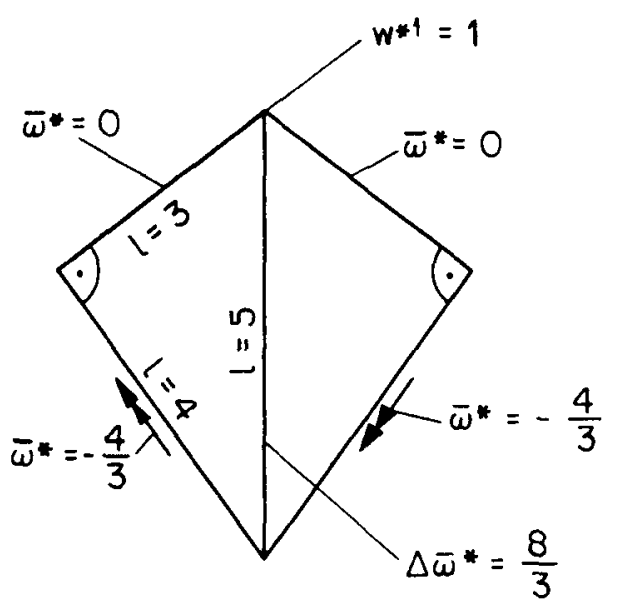

Bild B.7: Virtuelles Verschiebungsfeld

Aus den Bildern B.6 und B.7 ergibt sich die innere Arbeit zu

$$
\begin{aligned}
\underline{A}_{i}^{*} & =\sum_{c} \bar{m}_{n} \bar{\omega}_{n}^{*}+\sum_{d} \bar{m}_{n} \Delta \bar{\omega}_{n}^{*} \\
& =-\frac{55}{50} \cdot \frac{4}{3}-\frac{69}{100} \cdot \frac{4}{3}+\frac{3}{4} \cdot \frac{8}{3}=-\frac{179}{100} \cdot \frac{4}{3}+2,0=-\frac{29}{75}
\end{aligned}
$$

Der Index c läuft über die Randseiten, der Index d über die inneren Seiten.

Um die Gleichgewichtslasten ermitteln zu können, braucht man die Drillungsmomente $m_{n s}$ entlang der Randseiten. Sie berechnen sich aus

$$
m_{n s}=-m_{x} \sin \varphi \cos \varphi+m_{y} \sin \varphi \cos \varphi+m_{x y}\left(\cos ^{2} \varphi^{-} \sin ^{2} \varphi\right)
$$

und sind im Bild B.8 dargestellt.

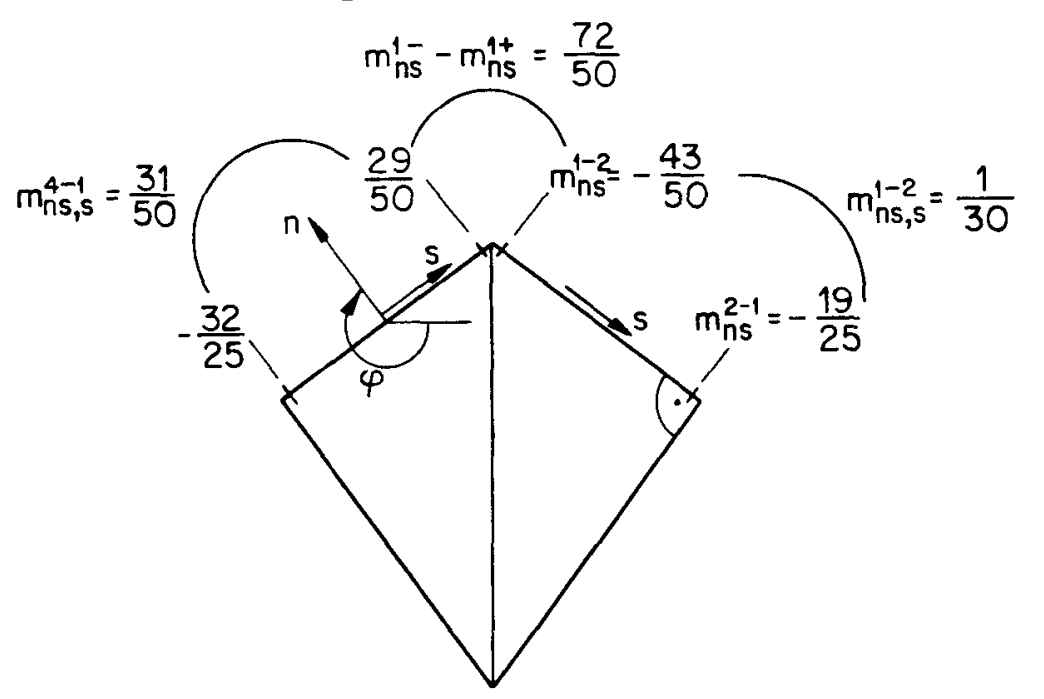

Beispiel: $\quad m_{n s}^{4-2}=0,5 \cdot \frac{12}{25}-2,0 \frac{12}{25}+0,5\left(\frac{9}{25}-\frac{16}{25}\right)=-\frac{43}{50}$

Bild B.8: Drillungsmomente entlang den Rondseiten 
Weiter müssen die Ableitungen $m_{n, n}$ berechnet werden. Sie sind aus dem Bild B.9 ersichtlich.

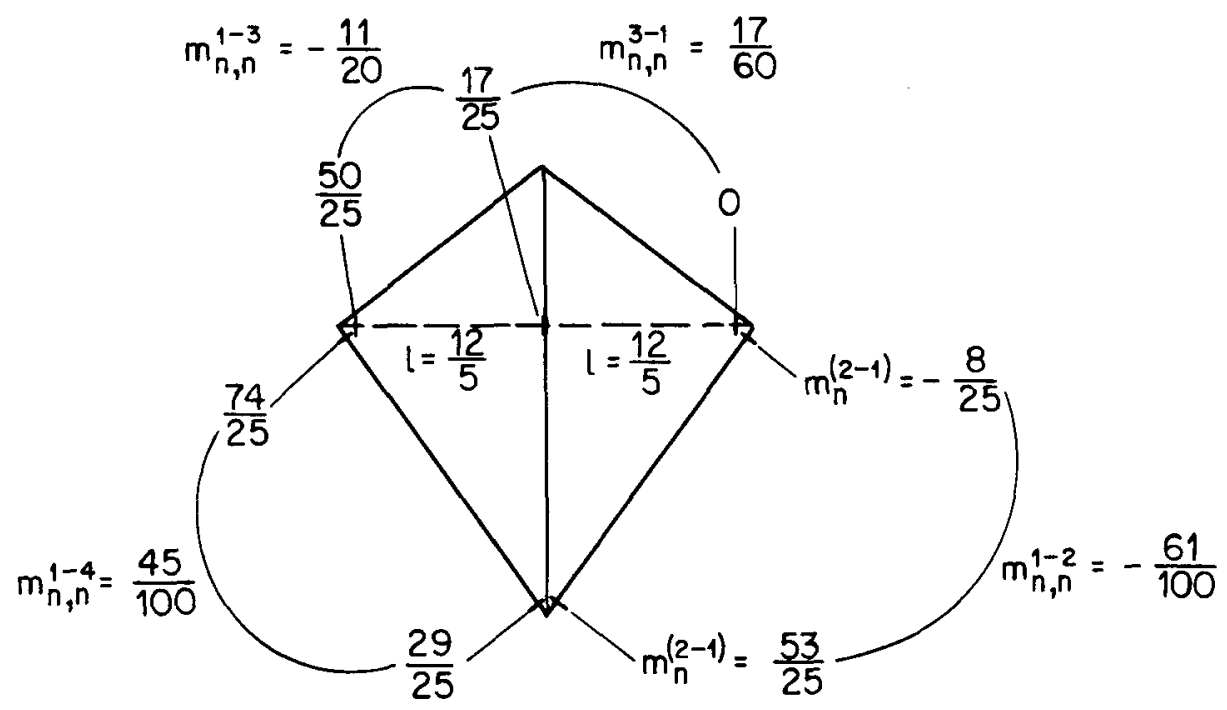

Bild B.9: Ableitungen $m_{n, n}$ entlong den Seiten

Die Gleichgewichtslasten für die Seiten 1-2, 1-4 und 1-3, die sich aus den Bildern B.8 und B.9 und der Gleichung (B.18) ergeben, sind im Bild B.10 dargestellt.

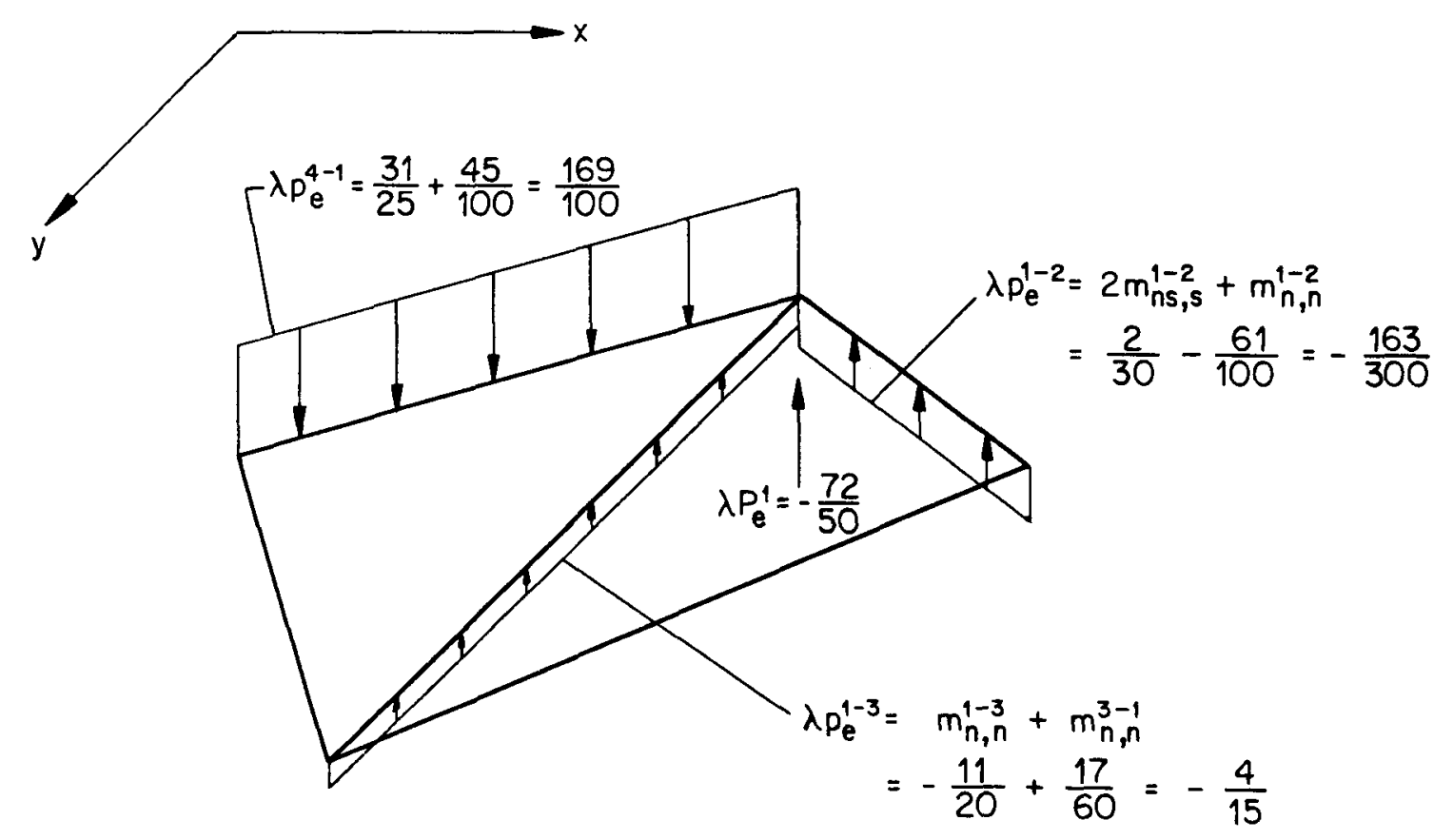

Bild B.10: Gleichgewichtslasten 
Aus diesen Gleichgewichtslasten erhält man die innere Arbeit

$$
\begin{aligned}
\underline{A_{i}^{*}} & =\frac{1}{2} \lambda \sum P_{e} l+\lambda P_{e}^{1} \\
& =\frac{1}{2}\left(\frac{169}{100} \cdot 3-\frac{163}{300} \cdot 3-\frac{4}{15} \cdot 5\right)-\frac{72}{50}=-\frac{29}{75}
\end{aligned}
$$

Das Resultat stimmt notwendigerweise mit dem Resultat (B.19) überein.

Zur Untersuchung des Modelles "TLC" wird die Gleichung (4.18) abgekürzt in Indexschreibweise geschrieben.

$$
-\iint_{F} m_{i j} w_{, i j}^{*} d F-\lambda \iint_{F} p w^{*} d F=0
$$

Es ist hier nicht zweckmässig, die Pole der virtuellen Krümmung $w^{*}, n n$ direkt durch partielle Integration zu beheben, weil im neuen Ausdruck (B.2) die Pole von $m_{n s, n}$ entlang der Seiten (Bild B.11) erscheinen würden.

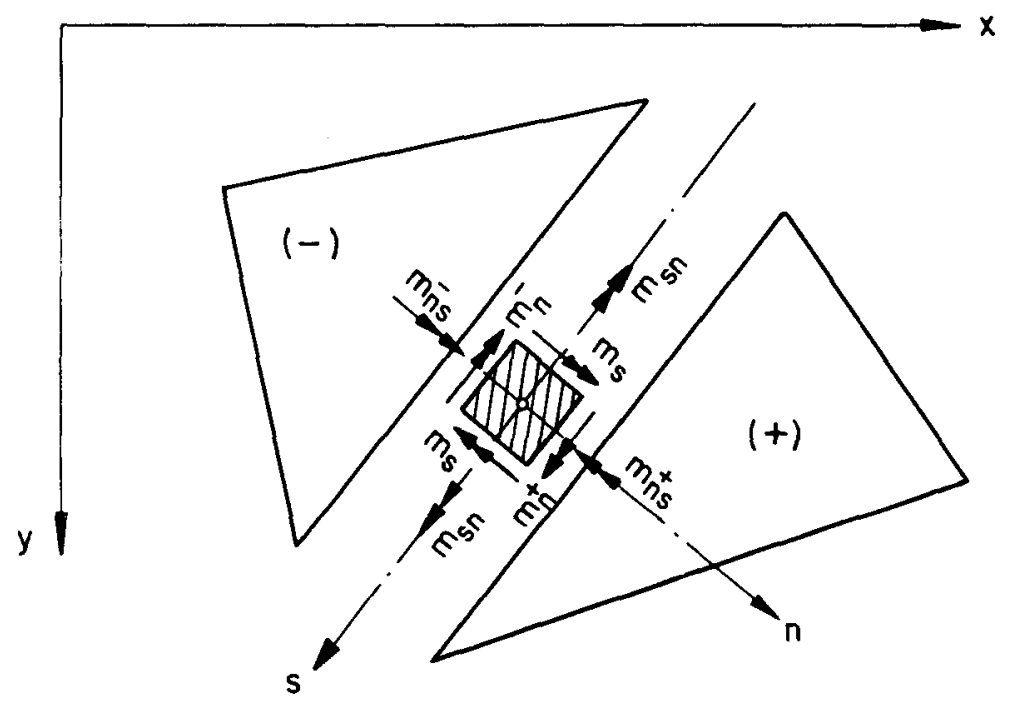

Bild B.11: Schnittkräfte entlang einer Seite

Die Mittelflächen $F^{e}$ der Elemente werden in drei beliebige Subdreiecke unterteilt. Als neue Integrationsgebiete werden die im Bild B.12 dargestellten Vierecke $F^{d}$ gewählt. Entlang des Randes verbleiben Dreiecke $F^{c}$ als Integrationsgebiete. Der Index d läuft über alle innern Seiten, der Index c über alle Randseiten. 


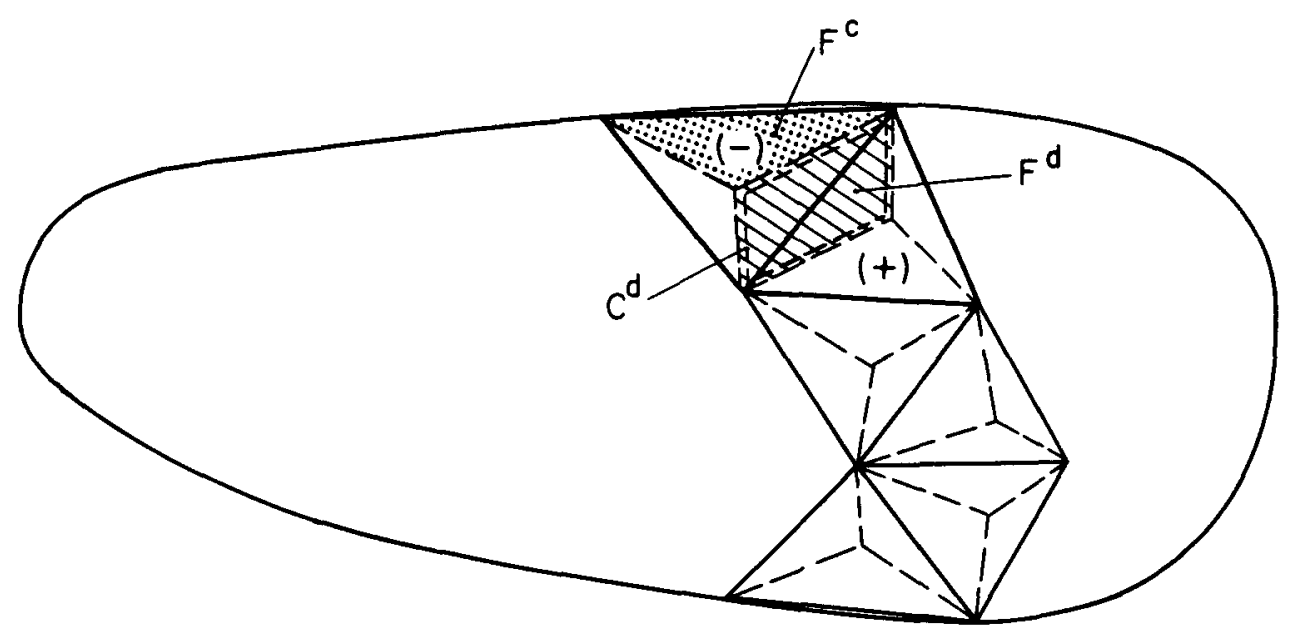

Bild B.12: Integrationsgebiete

Das erste Integral der Gleichung (B.21) wird innerhalb der Gebiete $F^{d}$ und $F^{C}$ in den Koordinaten der entsprechenden Seiten ausgedrückt. Entlang der gestrichelten Linien im Bild B. 12 weisen weder die Momente $m_{n}^{d}$, $m_{s}^{d}$ und $m_{n s}^{d}$, noch die virtuellen Krümmungen $w^{*}, n n{ }^{*} w^{*} d$, ns und $w^{*}$, ss Pole auf, so dass das Integral über $F$ durch die Summe der Integrale über die Gebiete $F^{d}$ und $F^{c}$ ersetzt werden kann.

$$
\begin{aligned}
-\iint_{F} m_{i j} w_{, i j}^{*} d F= & -\sum_{d} \int_{F^{d}}\left(m_{n}^{d} w_{, n n}^{* d}+2 m_{n s}^{d} w_{, n s}^{* d}+m_{s}^{d} w_{, s s}^{* d}\right) d F^{d} \\
& -\sum_{c} \iint_{F^{c}}\left(m_{n}^{c} w_{, n n}^{* c}+2 m_{n s}^{c} w_{, n s}^{* c}+m_{s}^{c} w_{, s s}^{* c}\right) d F^{c}
\end{aligned}
$$

In den Gebieten $F^{d}$ und $F^{c}$ sind $w^{*}$,ns und $w^{*}$,ss überall null. Die verbleibenden ersten Terme unter den Integralen werden entlang der Seiten unendich gross. Durch partielle Integration erhält man

$$
\begin{aligned}
-\iint_{F} m_{i j} w_{, i j}^{*} d F= & -\sum_{d}\left(\oint_{c^{d}} m_{n}^{d} w_{, n}^{* d} \nu_{n}^{d} d \eta-\iint_{F^{d}} m_{n, n}^{d} w_{, n}^{* d} d F^{d}\right) \\
& -\sum_{c}\left(\oint_{c^{c}} m_{n}^{c} w_{, n}^{* c} \nu_{n}^{d} d \eta^{d}-\iint_{F^{c}} m_{n, n}^{c} w_{, n}^{* c} d F^{c}\right)
\end{aligned}
$$

Die Flächenintegrale auf der rechten Seite der Gleichung (B.23) verschwinden, weil $m_{n, n}$ in $F^{d}$ und $F^{c}$ überall null ist. Die Koordinate $n$ und die Winkelfunktion $\nu_{n}$ sind im Bild B.13 dargestellt. 


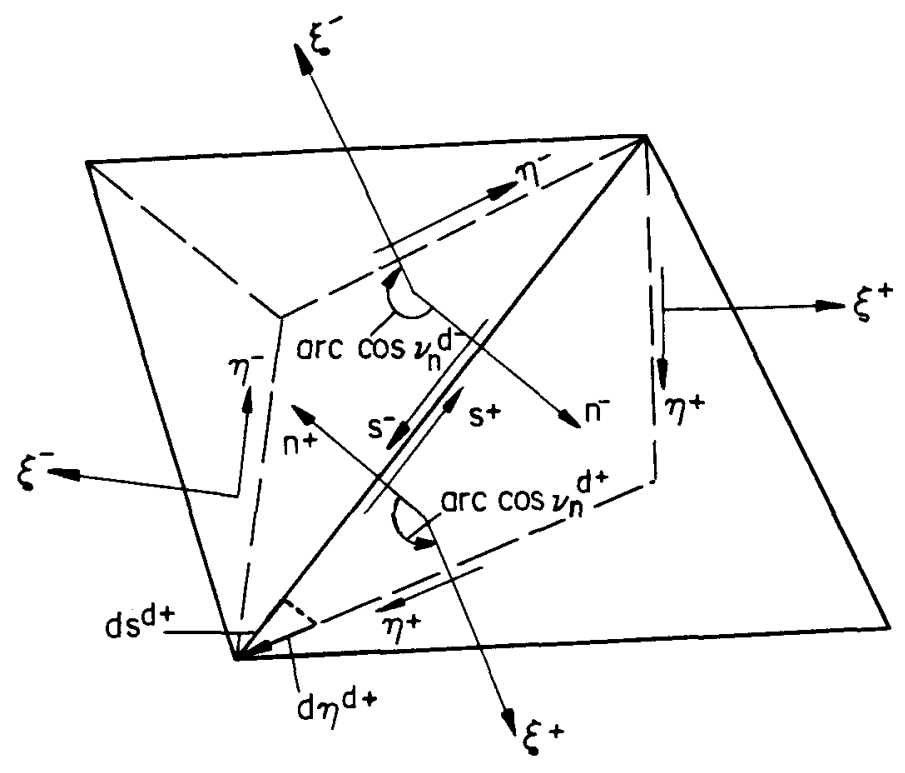

Bild B.13: Integration über Teilgebiet $F^{d}$

Die Integranden der Linienintegrale in der Gleichung (B.23) weisen keine Pole auf, so dass diese Integrale durch die Summe der Integrale über die Teilstücke $C^{d+}$ und $C^{d-}$ in den beiden Elementen ersetzt werden können. Beim Integral über $C^{C}$ entfällt der Beitrag der Randseite aufgrund der Randbedingung ( $w, n$ kann dort unabhängig variiert werden). Damit wird aus der Gleichung (B.23)

$$
\begin{aligned}
-\iint_{F} m_{i j} w_{i j j}^{*} d F= & -\sum_{d}\left(\oint_{C^{d+}} m_{n}^{d+} w_{, n}^{* d+} \nu_{n}^{d+} d \eta^{d+}+\oint_{C^{d-}} m_{n}^{d-} w_{, n}^{* d-} \nu_{n}^{d-} d \eta \eta^{d-}\right) \\
& -\sum_{c} \oint_{C^{c-}} m_{n}^{c} w_{, n}^{* c} \nu_{n}^{c} d \eta^{c}
\end{aligned}
$$

Aus dem Bild B.13 sieht man ferner, dass

$$
\begin{aligned}
& d s^{d+}=d \eta^{d+} \nu_{n}^{d+} \\
& d s^{d-}=d \eta^{d-} \nu_{n}^{d-} \\
& d s^{c}=d \eta^{c} \nu_{n}^{c}
\end{aligned}
$$

Da auch die übrigen Faktoren unter den Integralen konstant sind, lassen sich alle Linienintegrale als Integrale über die Seiten schreiben. Wenn wieder alle Integrale, die zu einem Element gehören, zusammengefasst werden, so ergitt sich schliesslich 


$$
\sum_{e} \oint_{c^{e}} m_{n}^{e} w_{, n}^{* e} d s^{e}-\lambda \iint_{F} p w^{*} d F=0
$$

Damit ist die Gleichung (4.6) auch für das Modell "TLC" verifiziert.

Es soll jetzt noch untersucht werden, ob die Gleichgewichtsbedingung (4.18) exakt erfüllt ist. Dazu bestimmt man die Gleichgewichtslasten, indem man die Gleichung (B.21) in Funktion von $w^{*}$ ausdrückt, d.h. partiell integiert.

$$
I_{1}=-\iint_{F} m_{i j} w_{, i j}^{*} d F=-\oint_{C}\left(m_{n} w_{, n}^{*}+m_{n s} w_{, s}^{*}\right) d s+\iint_{F} m_{i j, j} w_{, i}^{*} d F
$$

Der erste Term im Linienintegral verschwindet wegen der Randbedingungen. Der verbleibende Teil des Integrals kann als Summe über die Randseiten geschrieben werden, weil $m_{n s}{ }^{*}, s$ entlang $c$ keine Pole aufweist.

$$
I_{2}=-\oint_{C} m_{n s} w_{, s}^{*} d s=-\sum_{c} \int_{s_{0}^{c}}^{s_{b}^{c}} m_{n s}^{c} w_{, s}^{* c} d s
$$

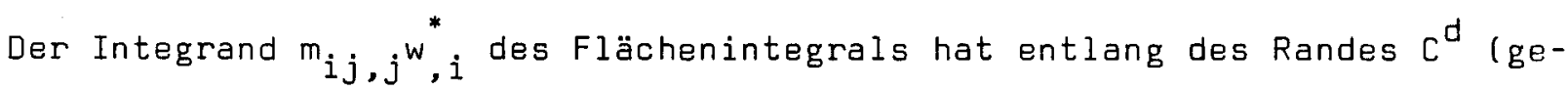
strichelte Linie im Bild B.12) keine Pole. Deshalb kann das entsprechende Integral

$$
I_{3}=\iint_{F} m_{i j, j} w_{, i}^{*} d F
$$

durch die Summe der Integrale über die Gebiete $F^{d}$ und $F^{C}$ ersetzt werden. Die Integranden werden in den Koordinaten der entsprechenden Seiten ausgedrückt.

$$
\begin{aligned}
I_{3}= & \sum_{d} \int_{F^{d}}\left(m_{n, n}^{d} w_{, n}^{* d}+m_{n s, s}^{d} w_{, n}^{* d}+m_{n s, n}^{d} w_{, s}^{* d}+m_{s, s}^{d} w_{, s}^{* d}\right) d F^{d} \\
& +\sum_{c} \int_{F^{c}}\left(m_{n, n}^{c} w_{, n}^{* c}+m_{n s, s}^{c} w_{, n}^{* c}+m_{n s, n}^{c} w_{, s}^{* c}+m_{s, s}^{c} w_{, s}^{* c}\right) d F^{c}
\end{aligned}
$$

Innerhalb der Gebiete $F^{d}$ und $F^{C}$ verschwinden alle Summanden bis auf die Terme $m_{n s, n}{ }^{w}, s^{*}$ Diese Terme werden entlang der Seiten unendlich gross und daher partiell integriert. 


$$
\begin{aligned}
& I_{3}=\sum_{d}\left[\oint_{C^{d}} m_{n s}^{d} w_{, s}^{* d} \nu_{n}^{d} d \eta^{d}-\iint_{F^{d}} m_{n s}^{d} w_{, n s}^{* d} d F^{d}\right] . \\
& +\sum_{c}\left[\underset{C^{c}}{\oint_{n s}} m_{, s}^{c} w_{n}^{* c} \nu_{n}^{c} d \eta^{c}-\iint_{F^{c}} m_{n s}^{c} w_{, n s}^{* c} d F^{c}\right]
\end{aligned}
$$

Die Flächenintegrale auf der rechten Seite verschwinden, weil ${ }^{*}{ }^{*}$, $n$ s innerhalb $F^{d}$ und $F^{c}$ null ist. Ebenso verschwinden die Umlaufintegrale über $C^{c}$, weil $m_{n s} w^{*}$,s in $F^{c}$ konstant ist. Die Linienintegrale über $C^{d}$ können durch die Summe über die Teilstücke $C^{d+}$ und $C^{d-}$ in den beiden Elementen ersetzt werden

$$
I_{3}=\sum_{d}\left[\int_{C^{d+}} m_{n s}^{d+} w_{, s}^{* d+} \nu_{n}^{d+} d \eta^{d+}+\oint_{c^{d-}} m_{n s}^{d-} w_{, s}^{* d-} \nu_{n}^{d-} d \eta^{d-}\right]
$$

Die Faktoren $\nu_{n}$ dn sind nach Gleichung (B.25) ebenso wie auch die übrigen Faktoren der Integranden konstant. Die Linienintegrale können also als Integrale über die Seiten geschrieben werden:

$$
I_{3}=-\sum_{d}\left[\int_{s_{a}^{+}}^{s_{b}^{+}} m_{n s}^{d+} w_{, s}^{* d+d s^{+}}+\int_{s_{0}^{-}}^{s b} m_{n s}^{d-} w_{, s}^{* d-} d s^{-}\right]
$$

Die Integrationsgrenzen sind im Bild B.14 dargestellt.

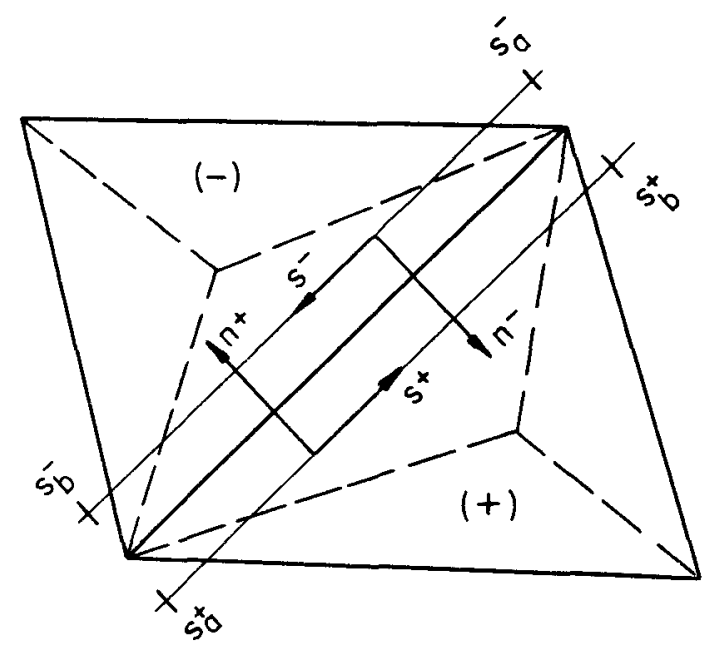

Bild B.14: Integrotionsgrenzen der Seiten 
Die Gleichung (B.27) kann geschrieben werden als

$$
I_{1}=I_{2}+I_{3}
$$

Schliesslich können die Linienintegrale über die inneren und die Randseiten partiell integriert werden

$$
-\int_{s_{a}}^{s_{b}} m_{n s} w_{, s}^{*} d s=-\left.m_{n s} w^{*}\right|_{s_{a}} ^{s_{b}}+\int_{s_{a}}^{s_{b}} m_{n s, s} w^{*} d s
$$

Das letzte Integral verschwindet, weil $\mathrm{m}_{\mathrm{ns}}$ entlang der Seiten kanstant ist. Man kann dann alle Summanden, die zum selben Knoten (zum selben w') gehören, zusammenfassen und erhält

$$
I_{1}=-\sum_{k}\left[\sum_{r}\left(m_{n s}^{k r b}-m_{n s}^{k r a}\right)\right] w^{* k}
$$

Der Index $k$ läuft über alle Knoten, der Index $r$ über alle an diesen Knoten anschliessenden Seiten. Die $m_{n s}^{r b}\left(m_{n s}^{r a}\right)$ sind die Drillungsmomente im Koordinatensystem der Seite $r$, bei welchen die Randkoordinate $s^{e}$ des zugehörigen Elementes gegen den Knoten (vom Knoten weg) läuft.

Setzt man die Gleichung (B.33) resp. (B.27) in die Gleichung (B.21) ein, so bekommt man

$$
-\sum_{k}\left[\sum_{r}\left(m_{n s}^{k r b}-m_{n s}^{k r o}\right)\right] w^{* k}-\lambda \iint_{F} p w^{*} d F=0
$$

Die Drillungsmomente können im Rahmen der Kirchhoff'schen Plattentheorie durch statisch äquivalente Schubkräfte ersetzt werden, so dass die Gleichgewichtslasten

$$
\lambda P_{e}^{k}=-\sum_{r}\left(m_{n s}^{k r b}-m_{n s}^{k r a}\right)
$$

konzentrierte Einzellasten in den Knoten sind.

Das Gleichgewicht (Gleichung (4.18)) kann also für das Modell "TLC" in Form der Normalmomente entlang der Seiten

$$
\sum_{e} \oint_{C^{e}} m_{n}^{e} w_{, n}^{* e} d s^{e}-\lambda \iint_{F} p w^{*} d F=0
$$


oder in Form der Gleichgewichtslasten (Gleichung (B.34)) ausgedrückt werden.

Die Berechnung der innern Arbeit soll am früher verwendeten Beispiel (Bild E.4) gezeigt werden.

Es sei das folgende Momentenfeld gegeben:

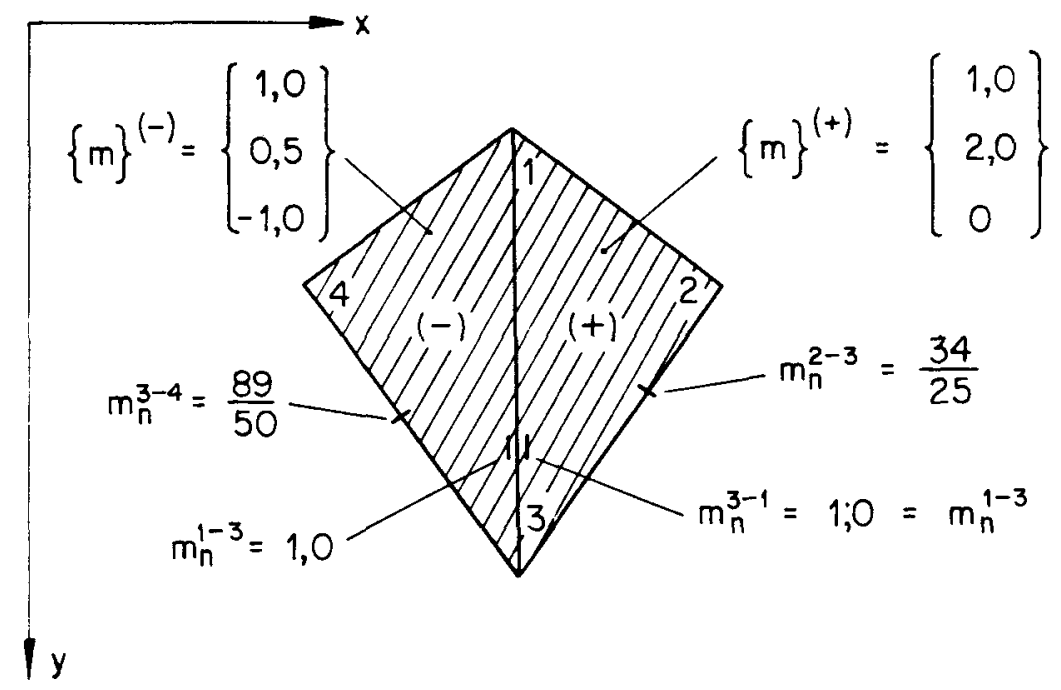

Bild B.15: Momentenfeld

Die konstanten Momente $m_{n}$ entlang der Seiten 3-4 und 2-3 berechnen sich nach der Gleichung (2.5). Die Rotationen infolge der virtuellen Verschiebung $w^{* 1}=1$ gehen wieder aus dem Bild B.7 hervor. Die innere Arbeit ist

$$
\begin{aligned}
\underline{A_{i}^{*}} & =\sum_{c} m_{n} \bar{\omega}_{n}^{*}+\sum_{d} m_{n} \Delta \omega_{n}^{*} \\
& =-\frac{89}{50} \cdot \frac{4}{3}-\frac{34}{25} \cdot \frac{4}{3}+1,0 \cdot \frac{8}{3}=-\frac{38}{25}
\end{aligned}
$$

Die Gleichgewichtslasten lassen sich aus den Drillungsmomenten entlang der Seiten ermitteln. 


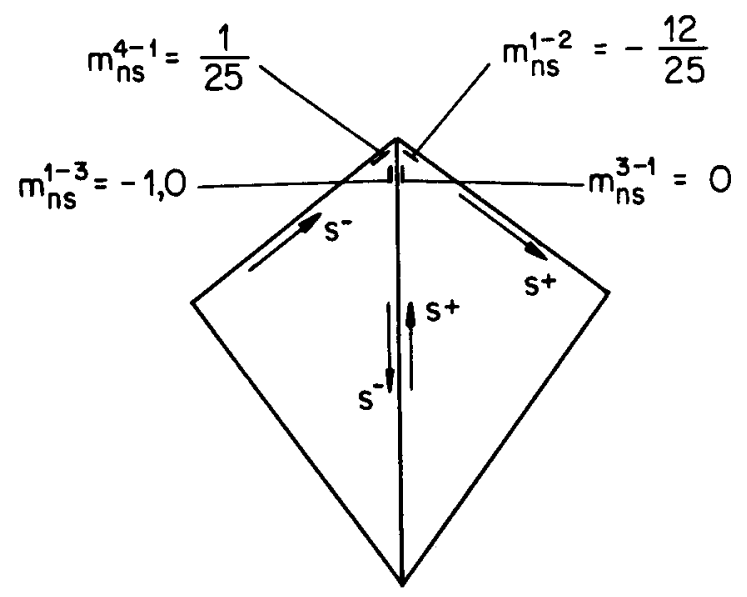

Beispiel: $\quad m_{n s}^{1-2}=1,0 \cdot \frac{12}{25}-2,0 \cdot \frac{12}{25}=-\frac{12}{25}$

Bild B.16: Drillungsmomente entlang der Seiten

Die Gleichgewichtslasten sind Einzellasten in den Knoten. Für die Berechnung von $A_{i}^{*}$ zählt nur diejenige im knoten 1.

$$
\lambda P_{e}^{1}=-\sum_{r}\left(m_{n s}^{r-1}-m_{n s}^{1-r}\right)=-\frac{1}{25}-1,0-\frac{12}{25}=-\frac{38}{25}
$$

Diese Knotenlast ist im Bild B.17 dargestellt.

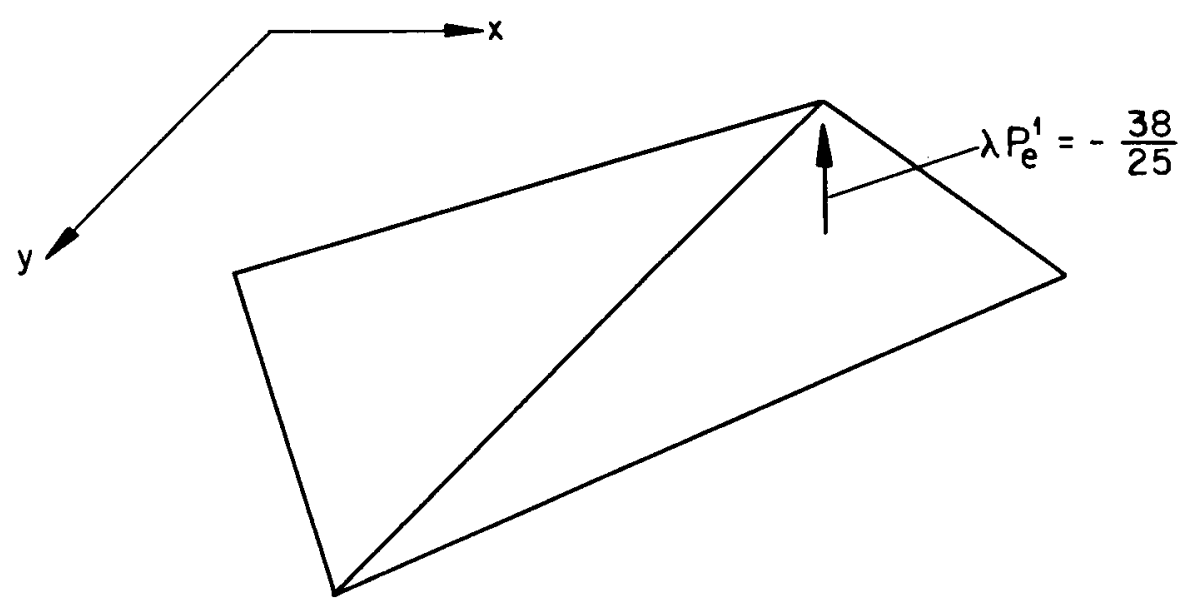

Bild B.17: Gleichgewichtslost 
Daraus ergibt sich die innere Arbeit zu

$$
\underline{A_{i}^{*}}=\lambda P_{e}^{1} \cdot 1=\underline{-\frac{38}{25}}
$$

Das Ergebnis stimmt also mit dem Resultat (B.36) überein. 
Bei der Mechanismusmethode [4] wird ein kinematisch zulässiges Geschwindigkeitsfeld angenommen. Der Quotient von Dissipationsleistung und Leistung der äussern Lasten ist ein oberer Grenzwert der Traglast. Da mit dem Modell "TLC" schon ein unterer Grenzwert bestimmt werden kann, wäre dieser obere Grenzwert wertvol1. Die Benützung der finiten Elemente hat den Zweck, die intuitive Wahl des ungünstigsten Mechanismus durch eine systematische Optimierung zu ersetzen.

Die Einteilung in finite Elemente und das Geschwindigkeitsfeld sind im Bild C. 1 dargestellt.

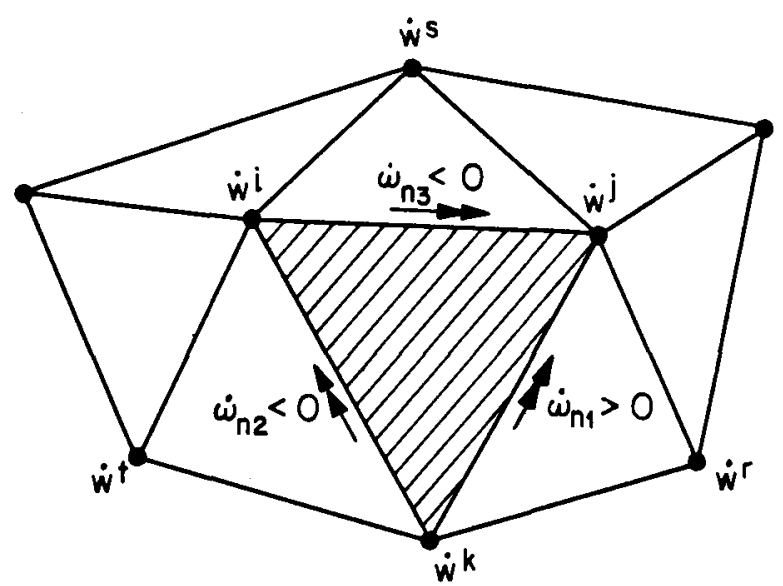

Bild C.1: Geschwindigkeitsfeld (Mechonismus)

Die $\dot{\omega}_{n}$ sind die entlang der Elementseiten konzentrierten Krümmungen. Es wird unterschieden zwischen $\dot{\omega}_{n}>0$ resp. $\dot{\omega}_{n}<0$, weil $\dot{\omega}_{n}$ bei der Berechnung der Dissipationsleistung mit dem plastischen Moment $P_{n}$ resp. mit $N_{n} z u$ multiplizieren ist. Es sei noch angenommen, die plastischen Momente entlang der einzelnen Seiten seien konstant.

Das duale lineare Programm muss folgende Bedingungen enthalten: 


$$
\begin{aligned}
\left\{N_{n}\right\} & \leqq\left\{m_{n}\right\} \leqq\left\{P_{n}\right\} \\
-[G]^{\top}\{\dot{w}\} & -[I]\left\{\dot{\omega}_{P}\right\}+[I]\left\{\dot{\omega}_{N}\right\}=\{O\} \\
\lambda & =\left\{P_{n}\right\}^{\top}\left\{\dot{\omega}_{P}\right\}+\left\{N_{n}\right\}^{\top}\left\{\dot{\omega}_{N}\right\} \\
1 & =\{P\}^{\top}\{\dot{w}\}
\end{aligned}
$$

In der ersten Zeile stehen die Plastizitätsbedingungen, in der zweiten die Kompatibilitätsbedingungen. [I] ist die Einheitsmatrix. Das lineare Programm ist im Tableau C.1 dargestellt. Die Matrix [G] ${ }^{\top}$ ist dieselbe wie diejenige in der Gleichung (6.22). Optimiert wird bezüglich der nichtnegativen Variablen $\dot{\omega}_{n}$.

Die Plastizitätsbedingungen von Gleichung ( $C .1$ ) entsprechen denjenigen für Stabtragwerke [24].

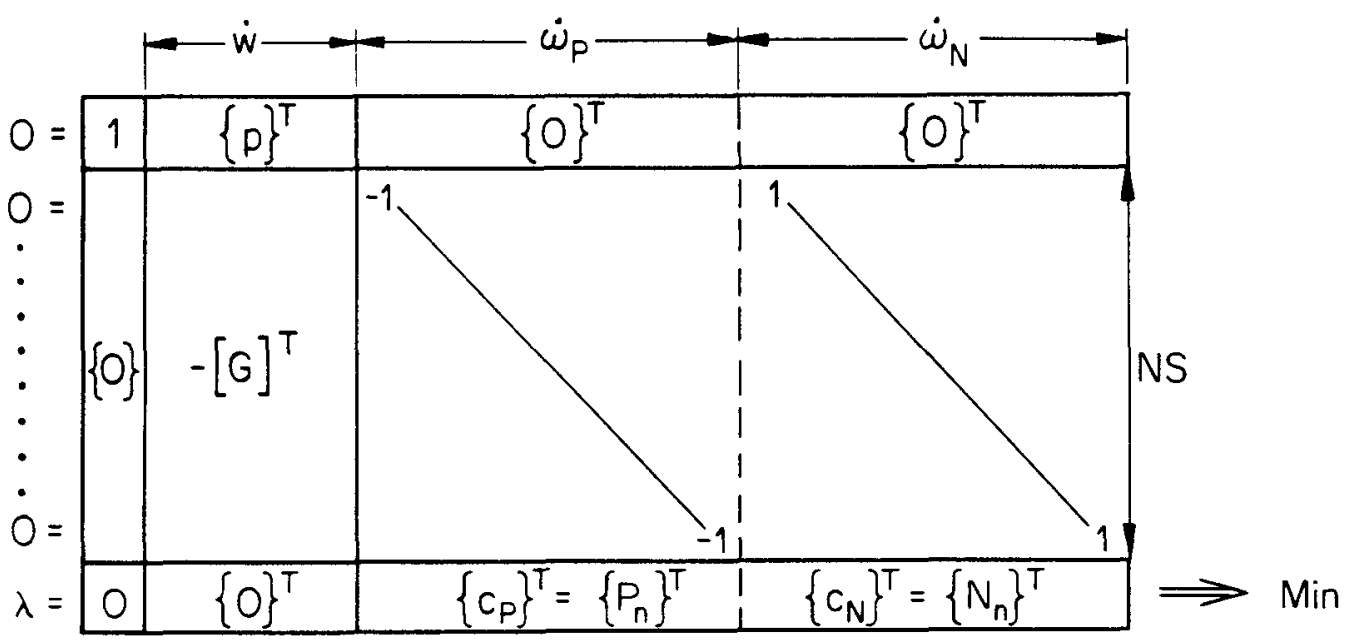

Tableau C.1: Lineores Programm für das duale Problem (Mechanismusmethode)

Das primaie Problem ist im Tableau C.2 dargestellt. 


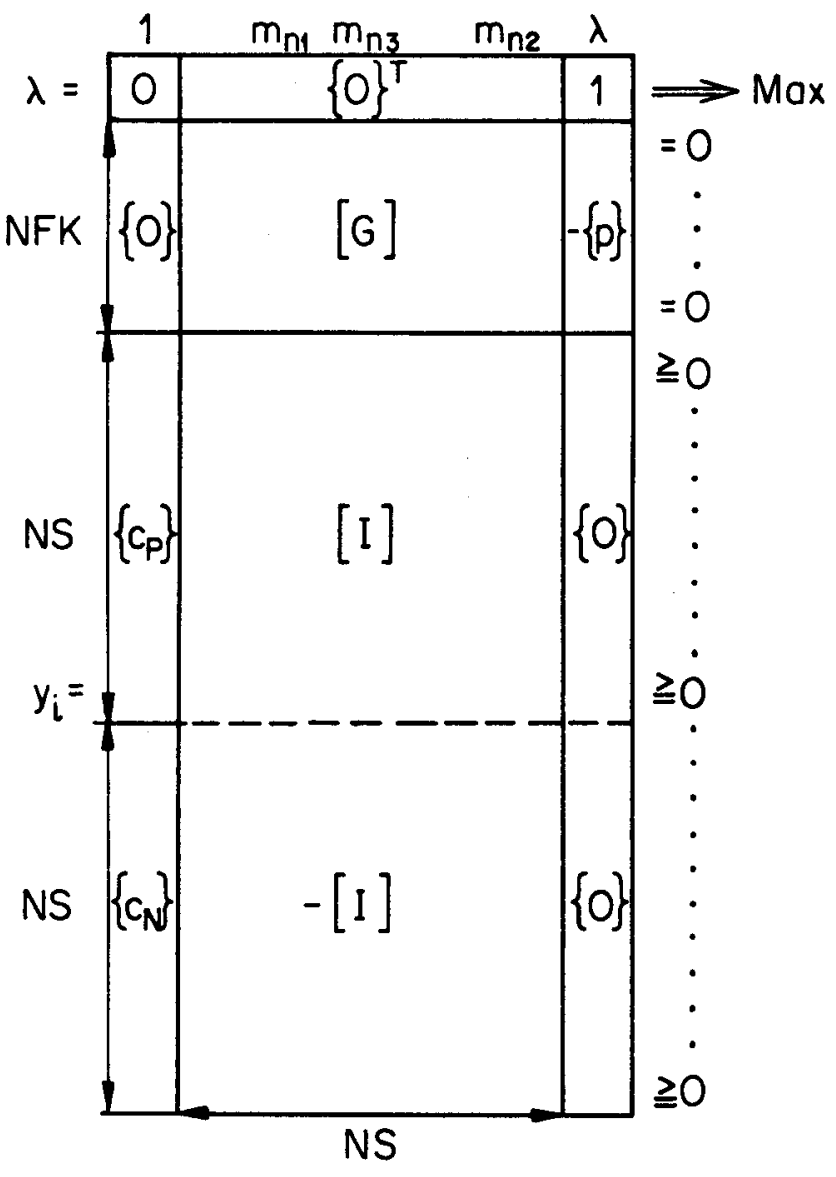

Tableau C.2: Lineares Programm für das primale Problem (Mechanismusmethode)

Es zeigt, dass das Gleichgewicht für die Knotenlasten $\lambda\{p\}$ erfüllt ist:

$$
[G]\left\{m_{n}\right\}-\lambda\{p\}=\{0\}
$$

Die Plastizitätsbedingungen werden in der Normalen zu den Elementseiten kontrolliert. An die Stelle der acht Plastizitätsbedingungen (2.15) treten die sechs Plastizitätsbedingungen

$$
\left\{N_{n}\right\} \leqq\left[T_{n}\right]\left\{m_{x-y}\right\} \leqq\left\{P_{n}\right\}
$$

Die Matrix $\left[T_{n}\right]$ ist in der Gleichung (5.1) definiert. Die Plastizitätsbedingungen für den Spezialfall $B_{1}=180^{\circ}, B_{2}=270^{\circ}$ (Bild C.2) lauten

$$
\begin{aligned}
& N_{1} \leqq m_{y} \leqq P_{1} \\
& N_{2} \leqq m_{x} \leqq P_{2} \\
& N_{3} \leqq m_{x} \sin ^{2} \beta_{3}+m_{y} \cos ^{2} \beta_{3}-2 m_{x y} \sin \beta_{3} \cos \beta_{3} \leqq P_{3}
\end{aligned}
$$




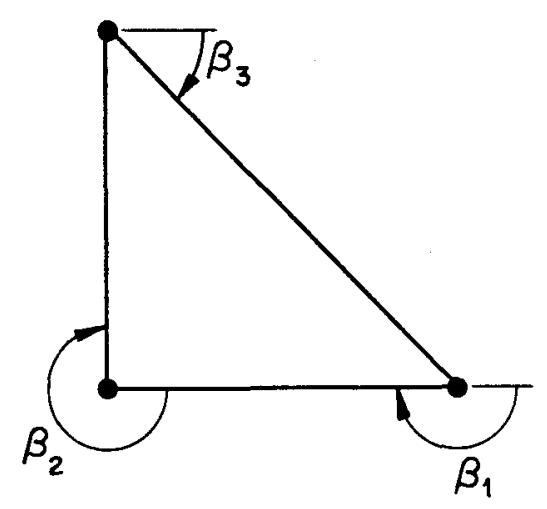

Bild C.2: Spezialfall eines Elementes

Die beiden ersten Zeilen der Gleichung (C.4) entsprechen der bekannten "quadratischen" Fliessbedingung, die dritte stellt zwei parallele Ebenen im $\left(m_{x}-m_{y}-m_{x y}\right)-$ Raum dar.

Für den Spezialfall $\beta_{3}=45^{\circ}$ vereinfacht sich die Gleichung (C.4) weiter zu

$$
\begin{aligned}
& N_{1} \leqq m_{y} \leqq P_{1} \\
& N_{2} \leqq m_{x} \leqq P_{2} \\
& \frac{1}{2}\left(N_{1}+N_{2}\right) \leqq \frac{1}{2}\left(m_{x}+m_{y}\right)-m_{x y} \leqq \frac{1}{2}\left(P_{1}+P_{2}\right)
\end{aligned}
$$

Die Plastizitätsbedingung (C.5) ist zusammen mit der Bedingung (4.31) mit [U] aus Gleichung (5.12) im Bild C.3 dargestellt. Man sieht, dass bei dieser Art Berechnung mittels finiter Elemente grobe Verletzungen der Plastizitätsbedingungen auftreten können. Die Kontrolle der Plastizitätsbedingungen ist von den $\beta_{i}$ und damit von der Einteilung in Elemente abhängig. Man erhält zwar einen obern Grenzwert der Traglast; es ist aber in der Regel ein relativ hoher, für praktische Berechnungen ungenügender Wert. 


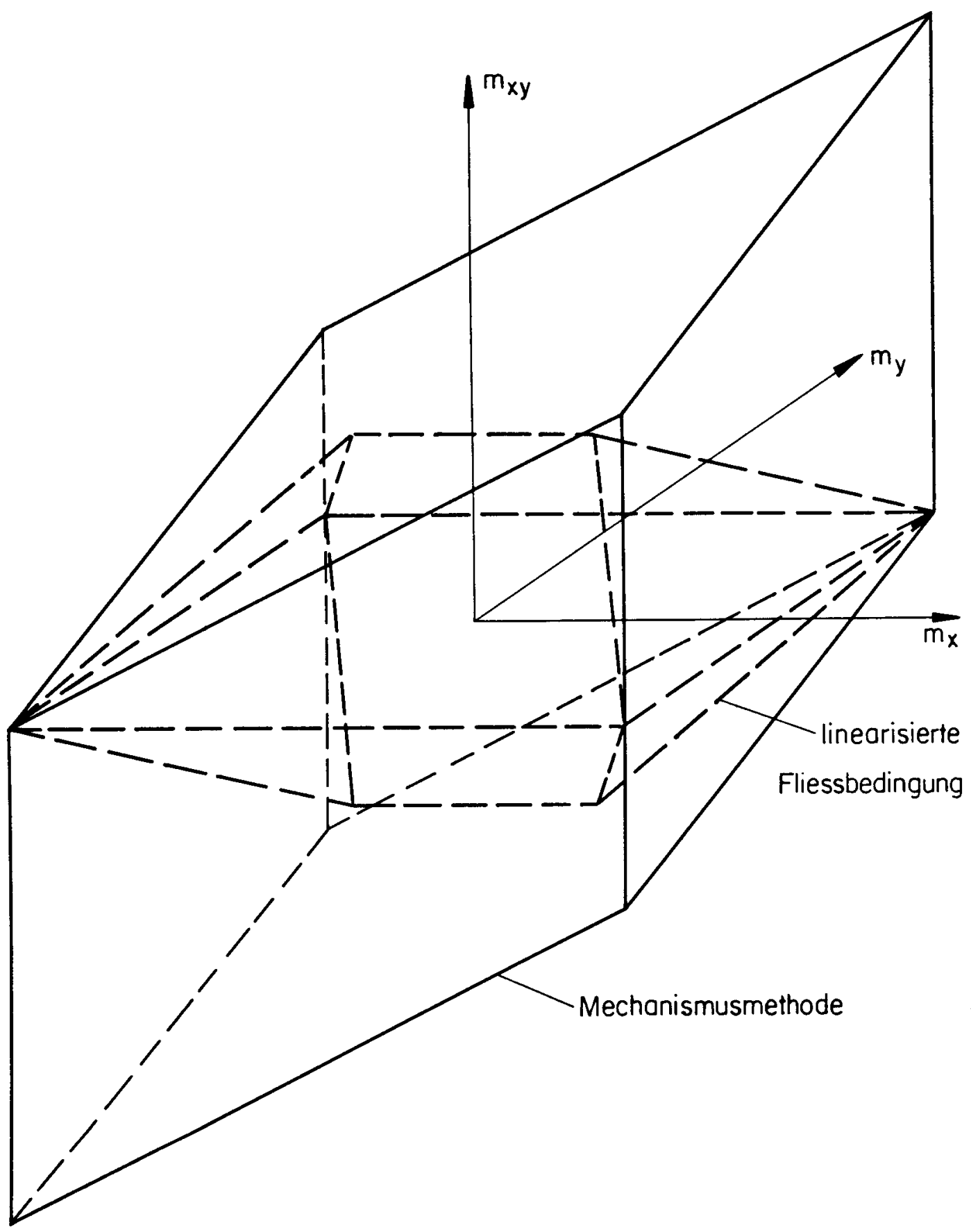

Bild C.3: Vergleich der Plastizitätsbedingungen

Die eingespannte Quadratplatte mit konstanter Armierung unter gleichmässig verteilter Belastung wurde mit einer sehr feinen und den effektiven Mechanismus nachahmenden Einteilung berechnet (Bild C.4). Der resultierende obere Grenzwert

$$
c_{\lambda} \cong 43,4
$$


kann als befriedigend bezeichnet werden, wenn man ihn mit den im Abschnitt 8.2 erhaltenen Resultaten vergleicht.

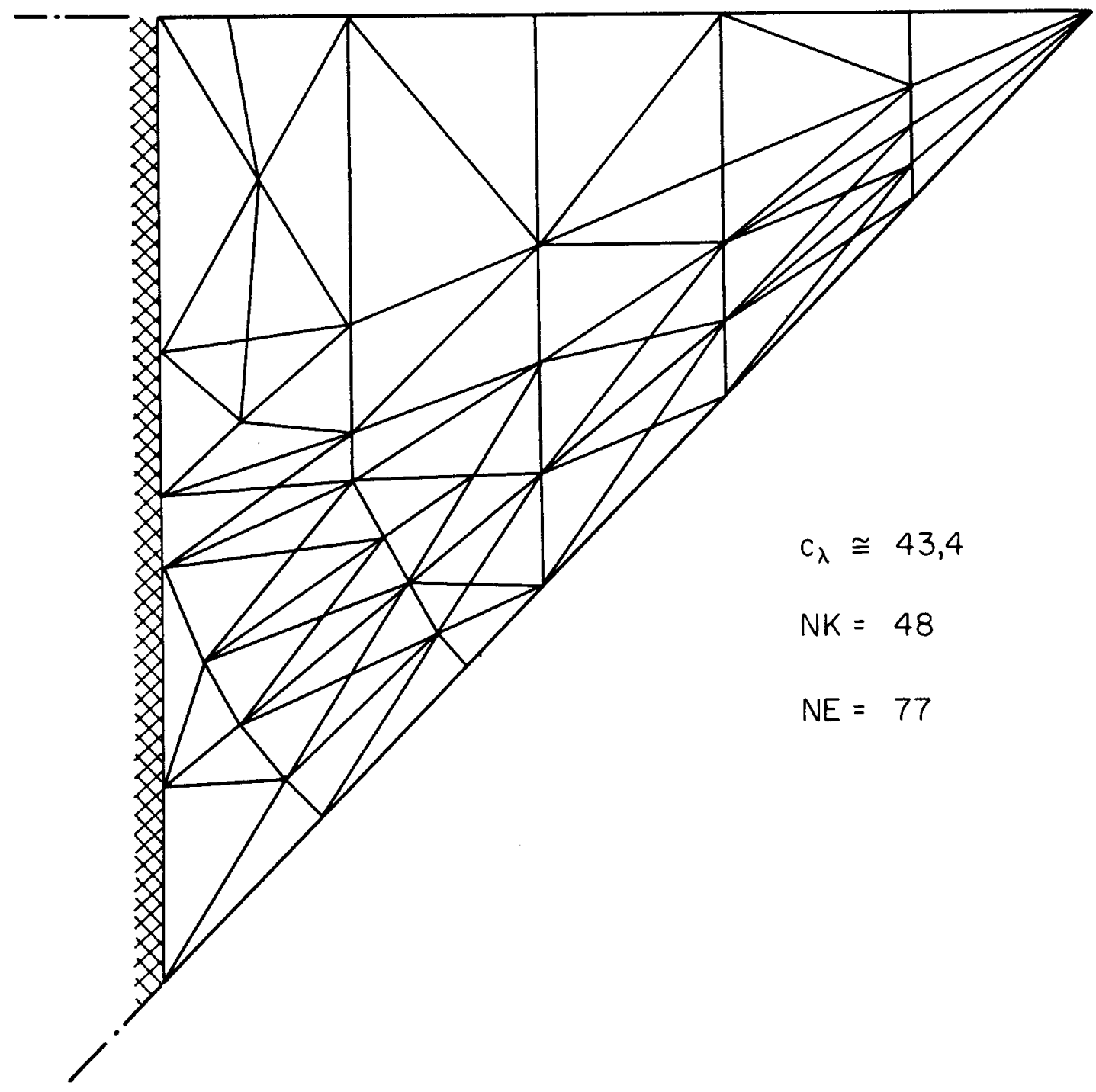

Bild C.4: Berechnung der eingespannten Quadratplatte (Mechanismusmethode)

Die Berechnung mit einer regelmässigen Einteilung und 64 Knoten ergab den relativ schlechten Wert $c_{\lambda}=45,8$. Bei der Mechanismusmethode wird das plastische Tragvermögen auf Drillung in unzulänglicher Weise erfasst. Deshalb wurde die Methode nicht weiter verfolgt. 
Hinweis auf die optimale Bemessung starr-plastischer Platten

Gegeben sei eine Platte, deren Verschiebungsfeld durch M Verschiebungsparameter und die Interpolationsfunktionen $\varphi_{m}(m=1, M)$ und deren Momentenzustand durch R Momentenparameter und die Interpolationsfunktionen $\psi_{r}$ ( $r=1, R$ ) approximiert werde. Gesucht sind die plastischen Widerstände, so dass für die Belastungsfälle $p^{l}(I=1, L)$ Gleichgewichtszustände existieren, welche die Plastizitätsbedingungen erfüllen, und so dass sie bezüglich einer gegebenen Zielfunktion optimal sind.

Aus der Definition (2.16) geht hervor, dass die plastischen Widerstände chq nicht voneinander unabhängig sind. Variable sind die Fliessmomente $P_{i j}$ und $N_{i j}(i=x, y ; j=x, y)$ in jedem Kontrollpunkt $q(q=1, Q)$. Bei praktischen Problemen ist es jedoch nicht sinnvoll, alle fliessmomente zu variieren, weil die Armierung über eine gewisse Strecke konstant gehalten werden soll. Deshalb wird ein Satz von positiven Fliessmomenten $f^{i}(i=1, F ; F \leq 3 Q)$ als unabhängige Variable definiert. Die plastischen Widerstände sind dann

$$
\{c\}=[c]\{f\}
$$

Weiter sollen die Fliessmomente nicht beliebige positive Werte annehmen können, sondern $H$ zusätzliche Beschränkungen

$$
\left\{y^{0}\right\}=[D]\{f\}-\{d\} \geqq\{0\}
$$

erfüllen.

Die Zielfunktion (z.B. eine linearisierte Kostenfunktion) sei gegeben durch

$$
z=\{z\}^{\top} \quad\{f\}
$$

Die Gleichgewichtsbedingungen lassen sich für jeden Lastfall als Gleichungssystem

$$
\{O\}=[G]\{m\}^{\prime}-\{P\}^{\prime}
$$


formulieren und die Plastizitätsbedingungen bilden ein Ungleichungssystem

$$
\{0\} \leqq\{c\}+[U]\{m\}^{l}
$$

für jeden Lastfall.

Aus diesen Bedingungen ergibt sich das lineare Programm für die optimale Bemessung

$$
\begin{aligned}
Z & =\{z\}^{\top}\{f\} \rightarrow \text { Minimum } \\
\{O\} & =[G]\{m\}^{b}-\{p\}^{b} \quad(l=1, L) \\
\{O\} \leqq[C]\{f\}+[U]\{m\}^{L} & (l=1, L) \\
\{O\} \leqq[D]\{f\} & -\{d\}
\end{aligned}
$$

Das lineare Programm ist im Tableau 0.1 dargestellt.

Wie bei der Berechnung der Traglast werden in einer ersten Phase die Gleichungen (Tableau 0.2 ) und in einer zweiten Phase die übrigen freien Variablen (Tableau 0.3) eliminiert. Die Optimierung beginnt mit der optimalen, unzulässigen Basislösung $Z=0$, weil die Koeffizienten der Zielfunktion als positiv vorausgesetzt werden dürfen und in der Konstantenkolonne negative Koeffizienten vorkommen.

Als optimale, zulässige Lösung erhält man schliesslich die gesuchten Fliessmomente $\{f\}$ und den Wert der Zielfunktion, sowie einen zulässigen Momentenzustand pro Belastungsfall.

Das hier angegebene Verfahren zur Berechnung der optimalen Armierung ist recht aufwendig. Der Aufwand könnte wahrscheinlich erheblich reduziert werden, wenn man das von Anderheggen [24, S. 70] vorgeschlagene "elastischplastische" Verfahren verwenden würde. Der Wert der Zielfunktion aus diesem Verfahren ist höher als derjenige aus der Berechnung des starr-plastischen Verhaltens. 


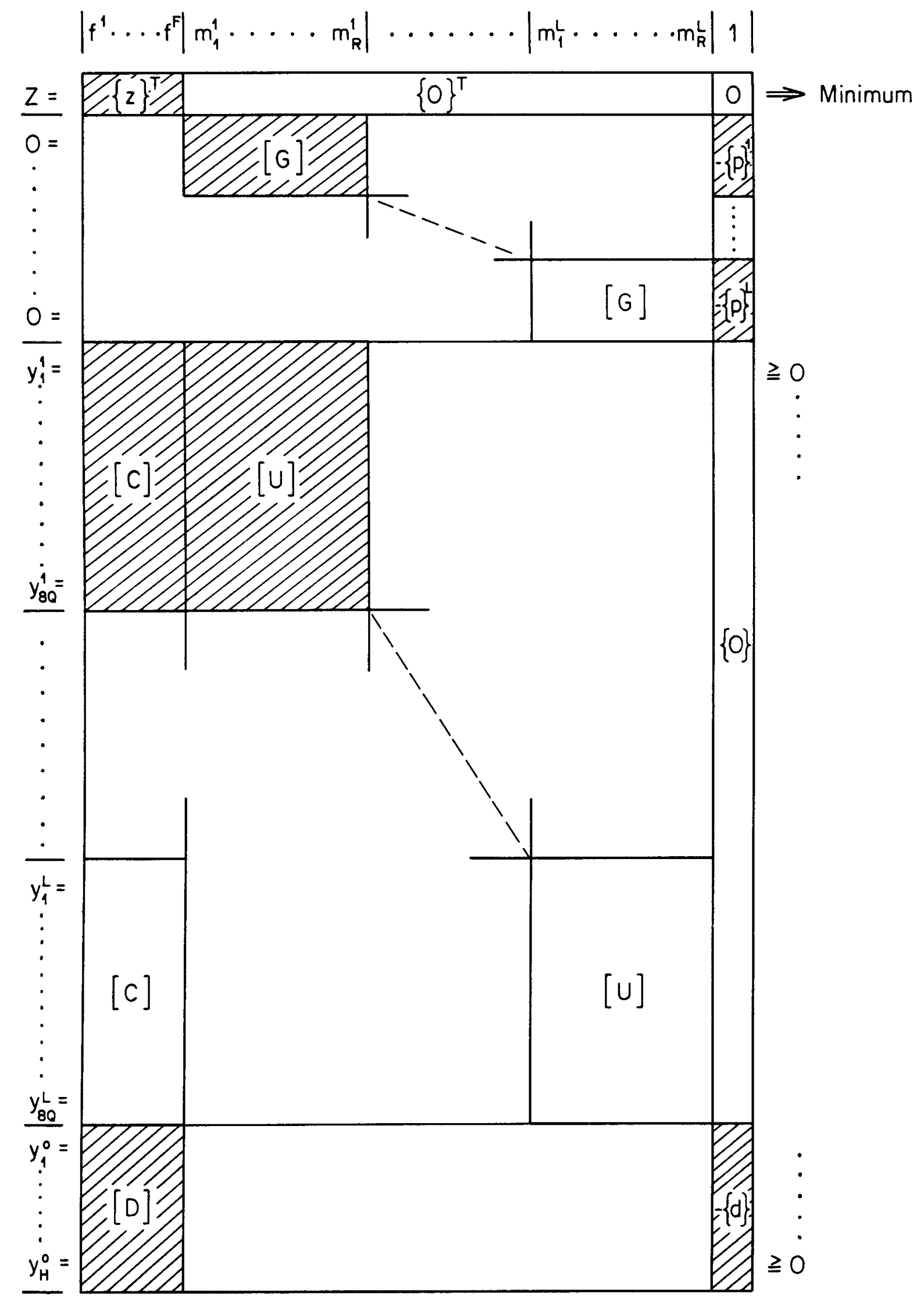

Tableau D.1: Ursprüngliches Tableau für die optimale Bemessung 


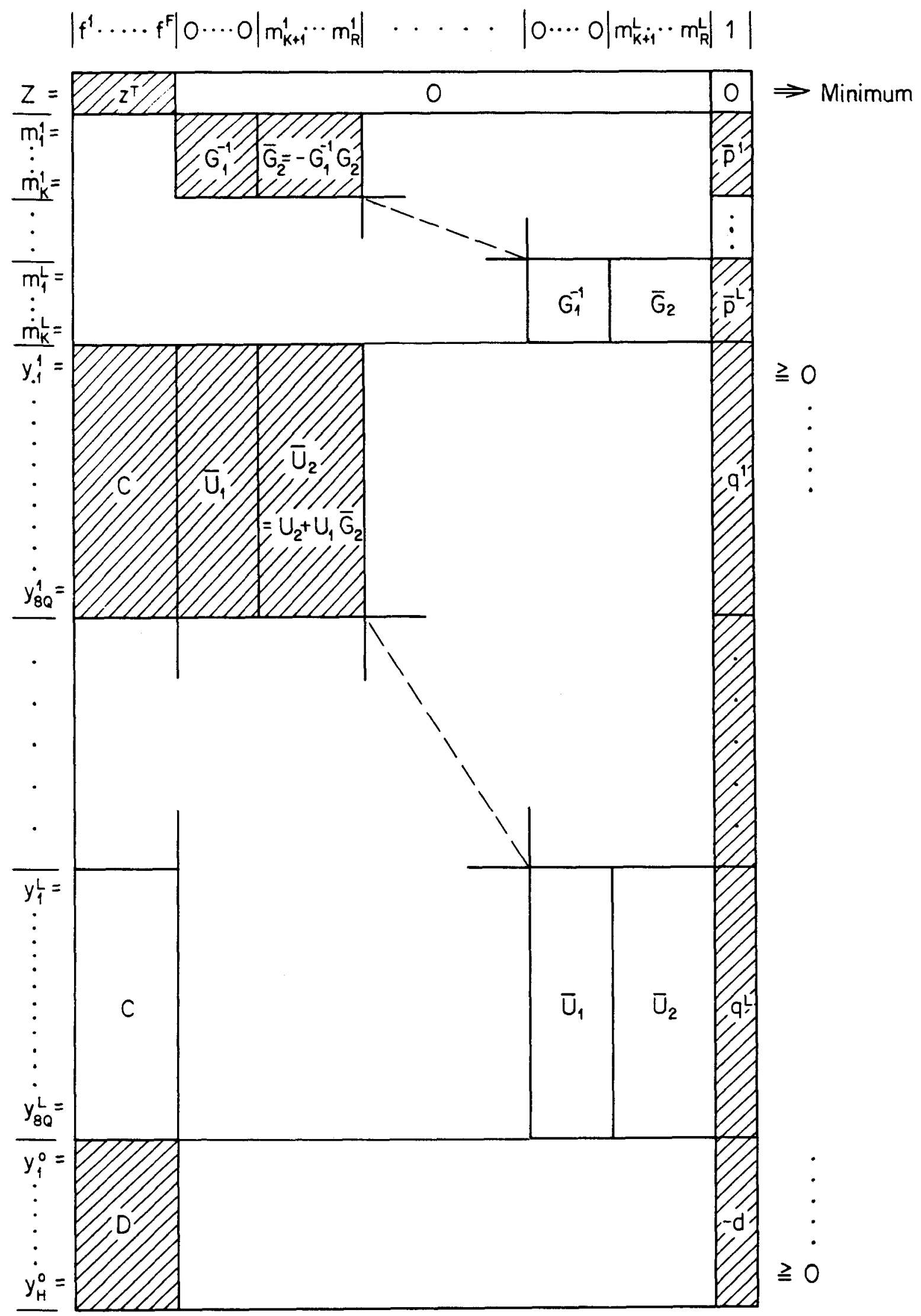

Tableau D.2: Geordnetes Tableau nach Elimination der Gleichungen 


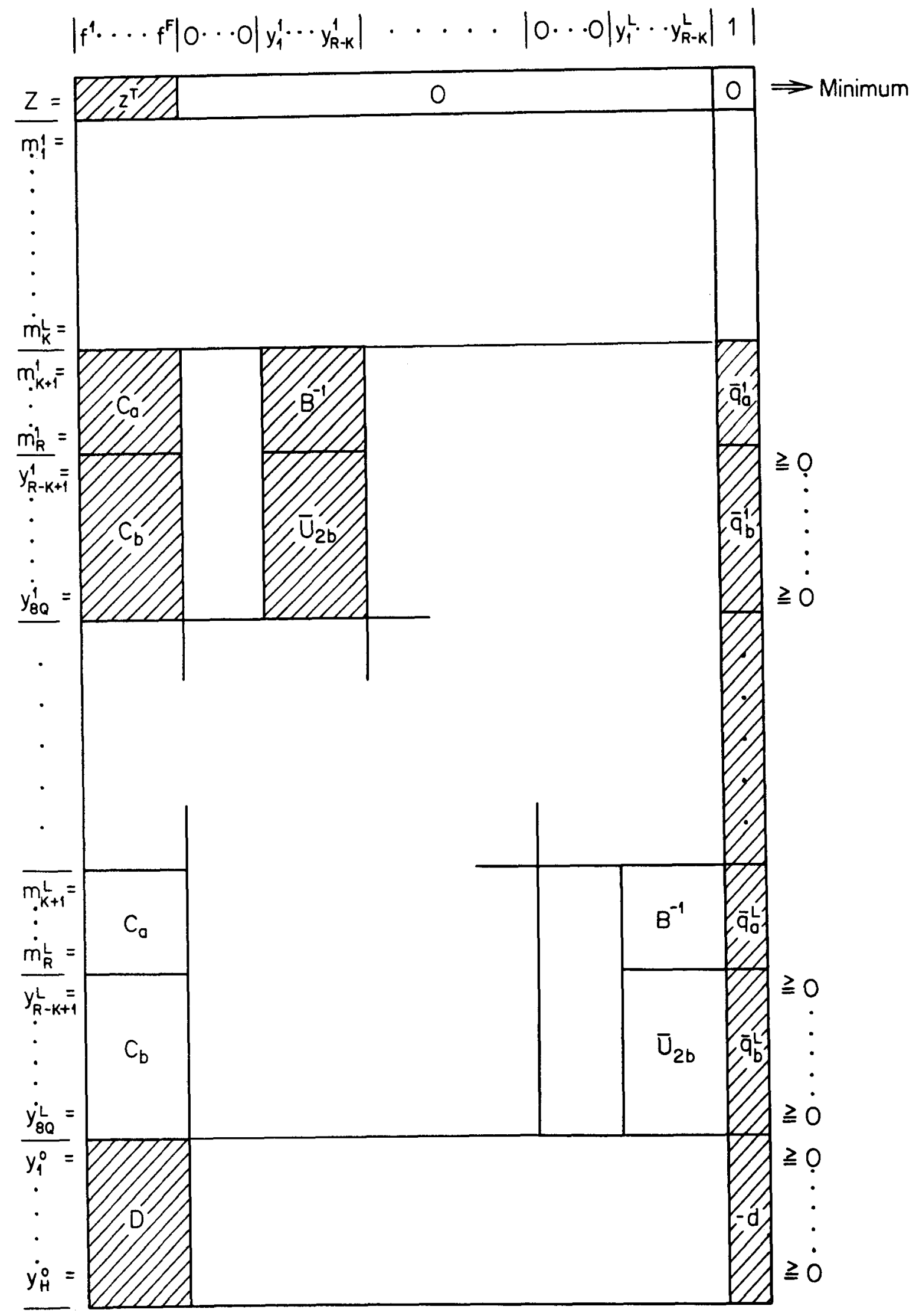

Tableau D.3: Geordnetes Tableau nach Elimination der freien Voriablen 
Das starr-plastische Verhalten von Stahlbetonplatten wird mittels der Methode der finiten Elemente als Iineares Optimierungsproblem berechnet. Dazu muss die verallgemeinerte Johansen'sche Fliessbedingung linearisiert werden.

Als wesentliche Angaben für die Anwendung der Methode der finiten Elemente sind die Parameter und der Verlauf der Interpolationsfunktionen für die Momente und die vertikalen Verschiebungen zu wählen. Die Beziehungen zwischen den Approximationsfunktionen für die Momente, denjenigen für die Verschiebungen und der äusseren Belastung müssen beachtet werden.

Zwei Modelle werden untersucht. Im einen verlaufen die Momente kontinuierlich und innerhalb der dreieckigen finiten Elemente linear; im anderen sind die Momente innerhalb der Elemente konstant und nur die Momente, die Normalspannungen senkrecht zu den Elementseiten erzeugen, haben einen kontinuierlichen Verlauf.

Die Maximumbedingung des Satzes über den untern Grenzwert, die Gleichgewichtsbedingungen und die Plastizitätsbedingungen ergeben ein System von Gleichungen und Ungleichungen, das mit der Methode der linearen Programmierung gelöst werden kann. Die Minimumbedingung des Satzes über den obern Grenzwert, die Kompatibilitätsbedingungen und die Normalisierungsbedingung für den Mechanismus bilden das dazu duale System.

Für jedes Modell wurde ein Computerprogramm entwickelt, das sich für praktische Berechnungen eignet. Als Optimierungsverfahren für die Berechnung mit dem Computer eignet sich ein spezielles, vom revidierten Simplexverfahren ausgehendes Verfahren, bei dem die Koeffizienten der Plastizitätsbedingungen nicht gespeichert werden. Die Berechnung von Beispielen, bei denen die genaue Lösung bekannt ist, und von Beispielen aus dem Bauwesen liefert gute Resultate.

Weitere Probleme, die auf ähnliche Weise gelöst werden könnten, sind: Die Berechnung von Platten mit Berücksichtigung der Scheibenkräfte und die optimale Bemessung von Platten. 
Le comportement rigide-plastique des dalles en béton armé est calculé comme problème d'optimalisation linéaire avec la méthode des éléments finis. Dans ce but le critêre de plastification de Johansen doit être généralisé et linéarisé.

Utilisant l'approximation avec des éléments finis, l'état des moments et des déplacements verticaux est représenté par un nombre limité de paramètres et de fonctions d'interpolation choisis. Les fonctions approximatives et la représentation des charges extérieures sont interdépendantes. Les éléments choisis sont triangulaires.

Deux modèles sont examinés. Le premier a des moments continus sur toute la dalle et linéaires dans chaque élément; pour le second modèle les moments sont constants dans chaque élément et seuls les moments dont le vecteur est parallèle aux côtés des éléments sont continus.

A partir de la condition maximum du théorème de la borne inférieure, ainsi que des conditions d'équilibre et des conditions de plastification linéarisées, on obtient un système d'équations et d'inéquations qui peut être résolu par l'algorithme simplex. De même, à partir de la condition minimum du théorème de la borne supérieure, des conditions de compatibilité et de l'équation normalisant le mécanisme, on obtient le système réciproque correspondant.

Des programmes pour le calcul électronique de problèmes pratiques ont été développés pour les deux modèles. Une version perfectionnée de l'algorithme simplex revisé permet de réduire la mémoire centrale nécessaire et le temps de calcul. Le calcul d'exemples dont on connalt la solution exacte ainsi que celui de cas tirés de la pratique de l'ingénieur donnent de bons résultats.

De la même manière, on peut résoudre le calcul de la charge limite des parois et des plaques avec effort de membrane et effectuer le dimensionnement optimum des dalles en béton armé. 
The rigid-plastic behaviour of reinforced-concrete slabs is calculated using the finite element method and linear programming. The generalized Johansen's yield condition must be linearized for that purpose.

Using the finite element approach, the state of moments and vertical displacements is represented by a discrete number of chosen parameters and interpolation functions. The approximative functions and the external load representation are dependent.

Two models are investigated. In one model the moments are distributed continuously and linearly within triangular finite elements; in the other model the moments are constant within triangular finite elements and only moments causing normal stresses, with reference to the element sides, are continuous.

From the maximum condition of the lower bound theorem, the equilibrium conditions and the linearized yield conditions follow a system of equalities and inequalities that can be solved using the simplex-algorithm. From the minimum condition of the upper bound theorem, the compatibility conditions and an equation scaling the mechanism follows the corresponding dual system.

Computer programs for engineering practice are developed for both models. A refined version of the revised simplex algorithm saves storage requirements and central processor time. The calculation of examples for which exact results exist, and of examples from engineering practice yield very satisfying results.

Other problems could be solved in a similar way: The calculation of ultimate loads for plate-stretching and combined plate-bending and -stretching and the optimum design of slabs. 


\begin{tabular}{|c|c|}
\hline B & Basismatrix \\
\hline G & Gleichgewichtsmatrix \\
\hline$I$ & Einheitsmatrix \\
\hline $\mathrm{L}$ & Boole'sche Matrix (logische Matrix) \\
\hline$T$ & Transformationsmatrix \\
\hline$\cup$ & Ungleichungsmatrix \\
\hline m & Moment pro Einheitslänge \\
\hline$m^{*}$ & virtuelles Moment pro Einheitslänge \\
\hline $\mathrm{P}$ & positives plastisches Moment \\
\hline $\mathrm{N}$ & negatives plastisches Moment \\
\hline Z & Zugkraft der fliessenden Armierung \\
\hline$p$ & Belastung \\
\hline $\bar{Q}$ & Reaktion \\
\hline$w$ & vertikale Verschiebung \\
\hline$w^{*}$ & virtuelle vertikale Verschiebung \\
\hline$\omega$ & Rotation \\
\hline$\dot{k}$ & konzentrierte Krümmungsgeschwindigkeit \\
\hline$\dot{\beta}$ & Komponente von $\dot{k}$ \\
\hline$\dot{K}$ & Krümmungsgeschwindigkeit \\
\hline$\dot{\alpha}$ & Komponente von $\dot{\kappa}$ \\
\hline$\varphi, \beta$ & Orientierung der Dreiecksseiten \\
\hline 1 & Seitenlänge \\
\hline$\alpha, \gamma$ & Orientierung der Armierung \\
\hline$\vartheta$ & Dreieckswinkel \\
\hline$A_{a}$ & äussere Arbeit \\
\hline$A_{i}$ & innere Arbeit \\
\hline$\lambda$ & Traglastfaktor \\
\hline C & plastischer Widerstand \\
\hline
\end{tabular}




\begin{tabular}{|c|c|}
\hline$x$ & freie Variable \\
\hline$y$ & Schlupfvariable \\
\hline$z$ & Zielfunktion \\
\hline $\mathrm{F}$ & Mittelfläche der Platte \\
\hline C & Rand \\
\hline$\varphi, \psi$ & Interpolationsfunktion \\
\hline$v$ & Normalenvektor von der Länge 1 \\
\hline$M$ & Anzahl Verschiebungsparameter \\
\hline $\mathrm{R}$ & Anzahl Momentenparameter \\
\hline NK & Anzahl Knoten \\
\hline NFK & Anzahl freier Knoten \\
\hline NS & Anzahl Seiten \\
\hline NE & Anzahl finiter Elemente \\
\hline$x, y, z$ & Koordinatenaxen \\
\hline$n, s$ & gedrehte Koordinatenaxen \\
\hline$\xi, \eta$ & gedrehte Koordinatenaxen \\
\hline 1,2 & taxen \\
\hline
\end{tabular}


[1] Wolfensberger, R.:

"Traglast und optimale Bemessung von Platten"

(Dissertation, ETH Zürich 1964)

[2] Jones, L.L. and Wood, R.H.:

"Yield line analysis of slabs"

(Thames and Hudson, Chatto and Windus, London 1967)

[3] Anderheggen, E.:

"Starr-plastische Traglastberechnungen mittels der

Methode der finiten Elemente"

(Institut für Baustatik, ETH Zürich 1971)

[4] Thürlimann, B. und Ziegler, H.:

"Plastische Berechnungsmethoden"

(ETH Zürich 1963)

[5] Stiefel, E.:

"Einführung in die numerische Mathematik"

(Teubner, Stuttgart 1961)

[6] Künzi, H.P. und Tan, S.T.:

"Lineare Optimierung grosser Systeme"

(Springer, Berlin 1966)

[7] Herrmann, L.R.:

"A Bending Analysis for Plates"

(Solid Rocket Operations TP 7 SRO, Sacramento 1965)

[8] Mansfield, E.H.:

"Collapse analysis of rigid-plastic plates"

(Proc. Roy. Soc., Series A, Vol. 241, 1957)

[9] Johansen, K.W.:

"Yield-line Theory"

(Cement and Concrete Association, London 1962) 
Ebner, F.:

"Ueber den Einfluss der Richtungsabweichung der Bewehrung von der Hauptspannungsrichtung auf das Tragverhalten von Stahlbetonplatten" (Dissertation, Karlsruhe 1963)

[11] Girkmann, K.:

"Flächentragwerke"

(Springer, Wien 1959)

[12] Hodge, P.G. and Belytschko, T.:

"Numerical Methods for the Limit Analysis of Plates" (Journal of Applied Mechanics, Dec. 1968)

[13] Clyde, D.H.:

"A yield criterion for reinforced concrete slabs" (Journal of the ACI, Discussion, Nov. 1967)

[14] Cardenas, A.E. and Sozen, M.A.: "Strength and behaviour of isotropically and nonisotropically reinforced concrete slabs subjected to combinations of flexural and torsional moments" (Structural Research Series No. 336, University of Illinois, Urbana 1968j

[15] Hatcher, D.S., Sozen, M.A. and Siess, C.P.: "Test of a reinforced concrete flat slab" (ASCE, Journal of the Structural Division, June 1969)

[16] Morley, C.T.:

"Optimum reinforcement of concrete slab elements against combinations of moments and membrane forces" (Magazine of Concrete Research, Sep. 1970)

[17] Herrmann, L.R.:

"Finite-element bending analysis for plates" (ASCE, Journal of the Engineering Mechanics Division, Oct. 1967)

[18] Sawczuk, A. und Jaeger, T.:

"Grenztragfähigkeits-Theorie der Platten" (Springer, Berlin 1963) 
[19] Zienkiewicz, O.C., Valliappan, S. and King, I.P.: "Elastoplastic solutions of engineering problems, 'initial stress', finite element approach" (Int. Journal for Num. Methods in Engineering, Vol. 1, 1969)

[20] Cervenka, V.:

"Inelastic Finite Element Analysis of Reinforced

Concrete Panels under In-plane Loads"

(Thesis, University of Colorado 1970)

[21] Nielsen, M.P.:

"Yield conditions for Reinforced Concrete Slabs" (Nordisk Betong, Copenhagen, 1963)

[22] Anderheggen, E.:

"Die Methode der finiten Elemente"

(Vorlesung, ETH Zürich 1971/72)

[23] Washizu, K.:

"Variational Methods in Elasticity and Plasticity"

(Pergamon Press, Oxford 1968)

[24] Anderheggen, E.:

"Optimale Bemessung von Stabtragwerken"

(Dissertation, ETH Zürich 1966)

[25] Ziegler, H.:

"Zwei Extremalprinzipien der irreversiblen Thermodynamik"

(Ingenieur-Archiv, Band 30, Heft 6, 1961)

[26] Stradal, 0.:

"Grundzüge der Planungsmethodik"

(Vorlesung, ETH Zürich 1971) 